A Characterization and Evaluation of Coal Liquefaction Process Streams

Quarterly Technical Progress Report July 1 through September 30, 1996

G. A. Robbins, S. D. Brandes,

R. A. Winschel

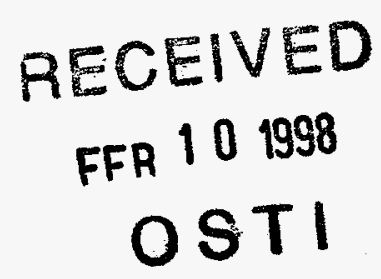

August 1997

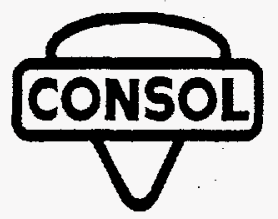

DHTABUTION OF THSS DOCUMENT IS UNMTED

CONSOL Inc.

Research \& Development

4000 Brownsville Road

Library, PA 15129

Under Contract to:

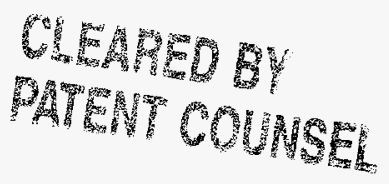

United States Department of Energy

Contract No. DE-AC22-94PC93054

T.S. DOE PATENT CLEARANCE IS NOT REQUTRED PRIOR

TO THE PUQLICANON OF THIS DOCUMENT 


\section{DISCLAIMER}

Portions of this document may be illegible electronic image products. Images are produced from the best available original document. 


\section{TABLE OF CONTENTS}

Page

EXECUTIVE SUMMARY

Characterization of Samples from HTI Run ALC-1

Recalibration of FTIR Spectroscopic Method for Phenolic -OH Determination

Oil Assays of HTI Run PB-03 Distillate Products

Resid Reactivity

INTRODUCTION

Contract Overview

Contract Activities this Period

Activities in Progress

RESULTS AND DISCUSSION

Characterization of Samples from HTI Run ALC-1 6

Introduction

Run Objectives and History

Run Plan and Operating History for ALC-1

General Sample Characteristics

SOH Oil Characteristics

Elemental Analysis of the $\mathrm{SOH}$ Water

Feed Slurry Characteristics

Concentrations of the Mo and $\mathrm{Fe}$ in Feed and Product Streams

Effect on Process Stream Properties of Feeding Coal Cleaned by Oil Agglomeration at Low $\mathrm{pH}$

Effect of the Decrease in Nominal Fresh Catalyst Addition Rate

Effect of Recycle Solvent Dewaxing and Hydrogenation

Wax Yields and Characteristics

Recalibration of FTIR Spectroscopic Method for Phenolic -OH Determination

Crude Oil Assays of Net Products of HTI Run PB-03

Resid Reactivity Studies

Resid Reactivity Tests $\quad 21$

Other Tests

\section{Table}

\section{LIST OF TABLES}

1 HTI Operating Conditions and Process Performance Results from Run ALC-1

2 Component Distribution of Whole Samples, HTI Run ALC-1

3 Proton Distribution of Whole Solids-Free Samples and Distillate Samples, Run ALC-1 
4 Proton Distribution of Filtered Whole Solids-Containing Samples and Resid Samples, HTI Run ALC-1 28

5 Phenolic -OH Concentration in Samples from HTI Run ALC-1 29

6 Microautoclave Conversions, HTI Run ALC-1 30

7 Composition of SOH Water Products from HTI Run ALC-1 30

8 Concentration of Components of the Feed Slurry Samples from HTI Run ALC-1 31

9 Ash Elemental Composition of Pressure-Filter Cakes from HTI Run ALC-1 31

10 Ash Elemental Composition of Feed Slurry $524{ }^{\circ} \mathrm{C}^{+}$Resids from HTI Run ALC-1 32

11 Mo and Fe Balance and Apparent Addition Rates for HTI Run ALC-1 33

12 Wax Content in VSOH, HTI Run ALC-1 34

13 Summary of ${ }^{1} \mathrm{H}-\mathrm{NMR}$ Analyses and Microautoclave Tests Relevant to Run ALC-1 Dewaxing Operations

14 Analysis and De-Oiling Yields of Wax from Run ALC-1, Period 25

15 CONSOL Analyses of PB-03 Crude Oil Assay Fractions 36

16 Resid Reactivity Tests 37

17 Comparison of Resid Conversion Data Obtained in $45 \mathrm{~mL}$ Microautoclaves (CONSOL R\&D) and Short Time Batch Reactor (University of Delaware) 44

18 Gas Chromatography Calibration Gases

\section{Figure}

\section{LIST OF FIGURES}

1 Component Distribution of Feed Slurry Samples from HTI Run ALC-1 46

2 Component Distribution of O-6 Bottoms Samples from HTI Run ALC-1 47

3 Component Distribution of Pressure-Filter Cake (PFC) Samples from HTI

Run ALC-1

48

Proton Aromaticity of Whole Process Stream Samples from HTI Run ALC-1 49

5 Paraffinic Proton Content of Whole Process Stream Samples from HTI

Run ALC-1

50

6 Donor Solvent Quality of Selected Whole and Distillate Samples from HTI

Run ALC-1

51

7 Phenolic -OH Concentration in Process Stream Samples from HTI Run ALC-1 52

\section{Appendix}

\section{LIST OF APPENDICES}

1 Recalibration of FTIR Spectroscopic Phenolic - $\mathrm{OH}$ Method

2 Sample Request for HTI Run PB-05

3 Crude Oil Assays of Net Products of HTI Run PB-03

4 University of Delaware Quarterly Report (7/16-10/15/96) 


\section{DISCLAIMER}

This report was prepared as an account of work sponsored by an agency of the United States Government. Neither the United States Government nor any agency thereof, nor any of their employees, makes any warranty, express or implied, or assumes any legal liability or responsibility for the accuracy, completeness, or usefulness of any information, apparatus, product, or process disclosed, or represents that its use would not infringe privately owned rights. Reference herein to any specific commercial product, process, or service by trade name, trademark, manufacturer, or otherwise does not necessarily constitute or imply its endorsement, recommendation, or favoring by the United States Government or any agency thereof. The views and opinions of authors expressed herein do not necessarily state or reflect those of the United States Government or any agency thereof. 


\section{Section 1}

\section{EXECUTIVE SUMMARY}

\section{CHARACTERIZATION OF SAMPLES FROM HTI RUN ALC-1}

CONSOL completed characterization of 64 samples from five run conditions of HTI Run ALC-1 (227-94), in which raw and cleaned (oil-agglomerated at low $\mathrm{pH}$ ) Black Thunder Mine subbituminous coal was fed and processed using only dispersed catalysts in the liquefaction reactors.

- Extraction of THF-soluble resid from the pressure-filter cakes was more complete when agglomerates were fed, leaving only $5 \%$ or less THF solubles in the extracted cakes. When raw coal was fed, the extracted cakes contained $9-34 \%$ THF solubles. HTI also observed improved filtration during the periods that agglomerates were fed. Improved operability, if verified by additional work, could be an economically significant benefit of coal cleaning by oil agglomeration at low $\mathrm{pH}$. An apparently higher Mo addition rate (see fourth bullet) may have contributed to the benefits of using oil-agglomerated coal.

- Other stream sample characteristics changed when oil-agglomerated coal was fed in Conditions 2-4, relative to when uncleaned coal was fed in Conditions 1 and 5 . The ash content of the O- 6 bottoms samples was lower when oil-agglomerates were fed. The THFsoluble $524^{\circ} \mathrm{C}^{+}$resid concentration in the feed slurry doubled when agglomerated coal was fed. Three factors may have influenced these characteristics. Higher coal conversion would have produced more resid. More efficient toluene-extraction of the filter cake would have recycled more resid. Removal of distillate as product to offset oil fed as part of the agglomerated coal would preferentially recycle the heaviest components.

- The analysis of process samples from Condition 5 indicates that the bench-unit dewaxing operations effectively removed paraffins from the vacuum distillate, and that the wax product is about $75 \%$ pure. The low concentration of wax in the feed minimized the impact of dewaxing on solvent quality improvement. The solvent hydrotreating operation effectively increased the hydroaromatic hydrogen content of the distillate and improved its solvent quality. 
- CONSOL determined the concentrations of Mo and $\mathrm{Fe}$ in feed slurry and pressure filter cake ash samples from Run ALC-1. The Mo and Fe concentration results were combined with HTI material balance data to calculate $\mathrm{Mo}$ and $\mathrm{Fe}$ balances and determine the apparent addition rates of $\mathrm{Mo}$ and $\mathrm{Fe}$ catalysts. The resulting $\mathrm{Mo}$ and $\mathrm{Fe}$ material balance closures were between $80-120 \%$. The results indicate that the Mo addition rate was inadvertently $10-169 \%$ higher than the target rate. The highest Mo addition rates were during agglomerated coal feed periods $(13,17,20)$. The results show a low Fe addition rate in Period 13 ( $39 \%$ of the target rate), and $4 \%$ to $36 \%$ higher than design rate in the other periods.

- Although the nominal fresh Fe and Mo catalyst addition rates decreased after Condition 2, there was an improvement in performance during Condition 3. The rate of $\mathrm{Fe}$ catalyst addition increased, based on the calculated $\mathrm{Fe}$ concentration from observed $\mathrm{Fe}$ concentrations in two streams. The observed performance improvement likely resulted from the increase in $\mathrm{Fe}$ catalyst concentration.

- Although HTI observed that the in-line hydrotreater (HTU) was less effective than expected in removing nitrogen and sulfur from the second-stage separator overheads (SOH), CONSOL data for all conditions show that this stream was effectively hydrogenated. It was hydrogenated to about the same degree as $\mathrm{SOH}$ produced in the coal-only operations during Run CMSL-9, and more hydrogenated than those produced in the coal-only operations during Run CMSL-11. The hydrotreated SOH oils were devoid of phenolic $-\mathrm{OH}$, in spite of high phenolic $-\mathrm{OH}$ concentration in the unhydrotreated first-stage $\mathrm{SOH}$ oils. These observations suggest that the hydrogenation and phenol removal effectiveness of the catalyst was minimally affected by a distillation system upset early in Run ALC-1 that seriously decreased the $\mathrm{N}$ and $\mathrm{S}$ heteroatom removal ability of the HTU catalyst.

- Feed slurry samples were found to contain less than $5 \% 343^{\circ} \mathrm{C}^{-}\left(650^{\circ} \mathrm{F}^{-}\right)$material, contrary to concerns that large amounts of light oil were being recycled.

- About $1 \%$ of the feed coal carbon reports to the separator overhead water product. Error resulting from omitting this stream from elemental balance calculations should be small. 
RECALIBRATION OF FTIR SPECTROSCOPIC METHOD FOR PHENOLIC -OH DETERMINATION

The Fourier-transform infrared (FTIR) spectroscopic method used to determine the phenolic -OH concentration in liquefaction samples was recalibrated and implemented on a new FTIR instrument. A new calibration was obtained from spectra of standard phenol compounds. An error estimate for each determination was added to the analysis software. The method was validated by comparison of results from samples run on the old and new systems.

\section{OIL ASSAYS OF HTI RUN PB-03 DISTILLATE PRODUCTS}

CONSOL arranged to have crude oil assays conducted on the net products of HTI Run PB-03, at $D O E$ 's request. Crude oil assays were conducted on net products obtained during periods of Run PB-03 for which the on-line hydrotreater was and was not used.

\section{RESID REACTIVITY}

- Resid reactivity tests were performed at CONSOL on the fifteen-resid sample set that the University of Delaware is using in their subcontract. The tests performed at CONSOL are described fully in the Results and Discussion section of this report.

- The University of Delaware made significant progress this quarter. Conversion values for all but two of the resids were obtained. The molecular structure model for coal-derived resids was assembled and is being optimized. A full description of the results is contained in the University of Delaware Quarterly Report appended to this report. 


\section{Section 2}

INTRODUCTION

This is the Technical Progress Report for the ninth quarter of activities under DOE Contract No. DE-AC22-94PC93054. It covers the period July 1 through September 30, 1996.

\section{CONTRACT OVERVIEW}

The objectives of this project are to support the DOE direct coal liquefaction process development program and to improve the useful application of chemical analyses to direct coal liquefaction process development. This project builds on work performed during DOE Contract No. DE-AC22-89PC89883. Independent analyses by well-established methods are obtained for samples produced in direct coal liquefaction processes under evaluation by DOE. New analytical instruments and techniques to examine coal-derived samples are being evaluated. The data obtained from this study are used to guide process development and to develop an improved data base on coal and coal liquids properties. A sample bank, established and maintained for use in this project, is available for use by other researchers. The reactivity of the non-distillable resids toward hydrocracking at liquefaction conditions (i.e., resid reactivity) is being examined. From the literature and experimental data, a kinetic model of resid conversion is being constructed. Such a model will provide insights to improve process performance and the economics of direct coal liquefaction.

\section{CONTRACT ACTIVITIES THIS PERIOD}

- Characterization of samples from HTI Run ALC-1 was completed. Results are described in this report.

- The phenolic $-\mathrm{OH}$ measurement method was recalibrated for the new FTIR system (Appendix 1).

- Sets of samples were requested from HTI Run PB-05 (Appendix 2).

- CONSOL arranged to have crude oil assays conducted on the net products of HTI Run PB-03, at DOE's request. Crude oil assays were conducted on net products obtained 
during periods of Run PB-03 in which the on-line hydrotreater was and was not in use. Results are provided in this report (Appendix 3 ).

- Resid reactivity tests were performed at CONSOL for a fifteen-resid sample set that the University of Delaware is investigating in their subcontract. The tests conducted at CONSOL are described in the Results and Discussion section of this report.

- The University of Delaware has made significant progress this quarter. Conversion values for all but two of the resids were obtained. The molecular structure model for coal-derived resids was assembled and is being optimized. A description of accomplishments is in the University of Delaware Quarterly Report appended to this report (Appendix 4).

\section{ACTIVITIES IN PROGRESS}

- Characterization work was started on samples from HTI Run PB-04. In HTI Run PB-04, Black Thunder Mine coal, Hondo resid, auto shredder residue, and other plastics were used as feedstocks.

- Plans were made to begin a literature search and experimental work on the synthesis of ethyl phenyl ethers (for high-octane oxygenate extenders for transportation fuels) from coal liquefaction phenols. Reagents were ordered.

- The University of Delaware began a parametric study of resid conversion. Variables are time and temperature. The study will initially focus on the reactivity of two resids (Wilsonville Run 259, V131B, and Wilsonville Run 260, V131B). 


\section{Section 3}

\section{RESULTS AND DISCUSSION}

\section{CHARACTERIZATION OF SAMPLES FROM HTI RUN ALC-1}

\section{INTRODUCTION}

CONSOL completed characterization of 64 samples from HTI Run ALC-1 (227-94), in which raw and cleaned Black Thunder Mine subbituminous coal was fed and processed using dispersed catalysts in the liquefaction reactors. The run consisted of 25 days of operation at five run conditions. Operating conditions and yields and performance data are provided in Table $1 .^{1-3}$ The run background is described briefly below. Additional run information is presented elsewhere. ${ }^{1-5}$

\section{RUN OBJECTIVES AND HISTORY}

There were four main objectives for Run ALC-1: 1) to provide a baseline operating period with Black Thunder Mine subbituminous coal, 2) to demonstrate liquefaction of low ash coal produced by low pH oil agglomeration of Black Thunder Mine coal, 3) to demonstrate liquefaction of Black Thunder Mine coal with dewaxing and hydrotreatment of distillate solvent, and 4) to operate with extinction recycle of $343{ }^{\circ} \mathrm{C}^{+}$material, such that a hydrotreated light distillate would be the intended net product. An additional objective was to exploit the advantage of feeding low-ash coal agglomerates by decreasing the fresh catalyst make-up rate.

HTI's bench liquefaction Run ALC-1 consisted of 25 days of operation. Major accomplishments were:

- Oil agglomeration reduced the ash content of Black Thunder Mine coal by $40 \%$, from $5.5 \%$ to $3.3 \%$ (MF, $\mathrm{SO}_{3}$-free ash basis).

- Excellent coal conversion (98\%) was obtained with oil agglomerated coal (about $3 \%$ higher than the raw Black Thunder Mine coal), which increased the potential product yield by $2-3 \%$ on an MAF coal basis.

- Agglomerates were liquefied with no handling problems. Filtration performance was improved. 
- Fresh catalyst (nominal) make-up rate was decreased by $30 \%$, with no apparent detriment to operations, when agglomerates were fed or when raw coal was fed (with solvent dewaxing and hydrotreating).

- Recycle solvent treatment by dewaxing and hydrotreating was demonstrated, but steady-state operation was not achieved.

- There was some success in achieving extinction recycle of the heaviest liquid products. This was evident in decreased resid yields and increased yields of light distillate during some run conditions.

- High yields of light distillates were obtained at several conditions during the run.

\section{RUN PLAN AND OPERATING HISTORY FOR ALC-1}

Run ALC-1 operating conditions, yields, and process performance results are shown in Table 1. The fresh catalyst addition rates for Conditions 1 and 2 matched those used in Run CMSL-9, Condition 6. The fundamental recycle strategy planned for Run ALC-1 was to recycle to extinction all materials that boil above $343^{\circ} \mathrm{C}$, except for the rejected, washed, pressure-filter cake. In practice, equipment and operability constraints did not allow this. The intent was to adjust space velocity to extinct the yield of $343^{\circ} \mathrm{C}^{+}$material. Pressure filtration was used to reject solids; the pressure-filter cake was washed with toluene to minimize rejection of solubles. The recycle solvent (including buffers) to MF coal ratio was 1.60 , and the recycle solids to MF coal ratio was $20 \%$. Both of these are higher than typically used in recent runs at HTI. The run ALC-1 conditions were designed to provide for high catalyst and unconverted coal recycling rates, which was intended to improve coal conversion, yet maintain operability with the high solids content in the recycle stream.

In Condition 1, the continuous atmospheric still bottoms (CASB) stream was filtered, and the recycle stream consisted of pressure-filter liquid (PFL) and a portion of the toluene-washed pressure-filter cake (PFC). The initial space velocity was $670 \mathrm{~kg} \mathrm{MF} \mathrm{coal} / \mathrm{h} / \mathrm{m}^{3}$. To shorten the solids recirculation time, it was decided near the end of Condition 1 to recycle unwashed PFC directly. 
In Conditions 2, 3, and 4, oil agglomerated coal was used as the feedstock. The agglomerating oil used to prepare the ALC-1 feed was heavy recycle distillate (V1074) from the Wilsonville pilot plant Run 263 end-of-run inventory. For Conditions 2, 3, and 4, it was necessary to reduce the quantity of distillate in the recycle by an amount equal to the agglomerating oil fed with the coal (as fed to liquefaction, the agglomerates contained about $23 \%$ oil). All of the PFL was sent to the vacuum still. All of the vacuum still bottoms (VSB) and some of the vacuum still overhead (VSOH) were recycled as necessary to meet total recycle requirements. A quantity of VSOH, approximately equal to the distillate agglomerating oil added with the coal, reported as product.

Early in Condition 2, the distillation conditions and flow scheme were changed to maintain operations with the constraints imposed by the run objectives. These changes resulted in a lighter recycle and heavier product than desired. The space velocity was decreased from $670 \mathrm{~kg}$ MF coal/ $/ \mathrm{h} / \mathrm{m}^{3}$ to $561 \mathrm{~kg} \mathrm{MF} \mathrm{coal} / \mathrm{h} / \mathrm{m}^{3}$ early in Condition 2. In Condition 3 (feeding agglomerates), the make-up catalyst rate was cut by $30 \%$, relative to Conditions 1 and 2 . Other distillation system changes were made to minimize recycle of light material. In Condition 4, the only operating condition changed was space velocity, which was decreased to $400 \mathrm{~kg} \mathrm{MF}$ coal/h/m to reduce the yield of $343^{\circ} \mathrm{C}^{+}$material.

The transition was made back to raw Black Thunder Mine coal in Condition 5. For this condition, which included dewaxing and hydrotreating, it was decided to keep the catalyst make-up rate at $70 \%$ of the Condition 1 value, and to set the space velocity at $481 \mathrm{~kg} \mathrm{MF}$ coal $/ \mathrm{h} / \mathrm{m}^{3}$ reactor. The entire PFL stream was vacuum distilled at $524^{\circ} \mathrm{C}$ to produce $\mathrm{VSOH}$ for dewaxing/hydrotreating. This material was ketone-dewaxed to remove paraffins, and the dewaxed oil then was hydrotreated to improve the donor solvent quality prior to recycle.

\section{GENERAL SAMPLE CHARACTERISTICS}

Sample characteristics are presented in Tables 2-6 and Figures 1-7. The aromatic hydrogen content of several whole liquid sample streams or filtered solids-containing whole sample streams is shown in Figure 4. Numerous changes made to distillation conditions and configurations during the run prevent direct comparison of vacuum distillate and pressure-filter liquid (PFL) streams. The streams shown in Figure 4 increase in aromaticity in the following order: 
stage 2 separator overhead $(\mathrm{SOH})$ oil < stage $1 \mathrm{SOH}$ oil < feed slurry = interstage slurry < $0-6$ bottoms $=$ pressure-filter cake $(P F C)$.

During the run, period-to-period variation in aromatic $\mathrm{H}$ content was generally small (Figure 4). However, for the feed slurry and interstage slurry streams, the Period 5-6 samples contained about 5\% to $8 \%$ more aromatic $H$ than the Period 13-21 samples, which in turn contained about $5 \%$ more aromatic $\mathrm{H}$ than did the Period 25 samples. The aromatic $\mathrm{H}$ differences are probably associated with use of raw (Periods 1-6 and 21-25) vs. oil-agglomerated (Periods 7-20) coal, use of different space velocities in several run periods (space velocity was changed three times during the run), and use of recycle solvent dewaxing and hydrogenation in Periods 21-25. Analogous, but inverted, trends were observed for the corresponding paraffinic $\mathrm{H}$ content of these same samples (Figure 5). The same pattern of high aromatic $H$ content in the Period 5-6 samples, intermediate aromatic $\mathrm{H}$ content in the Period 13-20 samples, and lower aromatic $\mathrm{H}$ content in the Period 25 samples also holds for other sample types (Table 3), such as the IBP $-454{ }^{\circ} \mathrm{C}\left(\mathrm{IBP}-850^{\circ} \mathrm{F}\right.$ ) distillate portion of the O-6 bottoms samples, and the $454-524{ }^{\circ} \mathrm{C}$ $\left(650-975^{\circ} \mathrm{F}\right)$ distillate portion of the feed slurry samples. The corresponding $1 \mathrm{BP}-454{ }^{\circ} \mathrm{C}$ (IBP-850 ${ }^{\circ} \mathrm{F}$ ) portion of the $0-6$ bottoms samples, and the $454-524^{\circ} \mathrm{C}\left(650-975^{\circ} \mathrm{F}\right)$ resid portion of the feed slurry samples show higher aromatic $\mathrm{H}$ in period 6 , but nearly equivalent aromatic $\mathrm{H}$ for periods 13-25 (Table 4).

The phenolic $-\mathrm{OH}$ concentration of process streams (Table 5, Figure 7) decreased according to the trend:

first stage $\mathrm{SOH}>$ feed slurry $=0-6$ bottoms $=$ PFC $>$ product $\mathrm{SOH}$ (second stage $\mathrm{SOH}$ )

No phenolic $-\mathrm{OH}$ was detected in the second-stage $\mathrm{SOH}$ samples. The phenolic $-\mathrm{OH}$ concentration in the feed slurry, O- 6 bottoms, and PFC streams decreased slightly as the run progressed. The phenolic $-\mathrm{OH}$ concentration in the first stage $\mathrm{SOH}$ increased after period 6 , and then decreased for the remainder of the run. It is not clear why this particular trend is observed. The large increase after period 6 may be related to the increase in resid concentration in the feed slurry while agglomerates were fed (see later section). It was observed that the first stage $\mathrm{SOH}$ phenolic - $\mathrm{OH}$ concentration was inversely proportional to $\mathrm{H}_{2}$ consumption on a MAF basis ( $\% \mathrm{H}_{2}$ 
consumption $=-2.40^{*}$ phenolic $-\mathrm{OH}$ concentration +11.2, standard error of $Y_{\text {est }}=0.13, R^{2}=$ 0.981).

\section{SOH OIL CHARACTERISTICS}

As expected for normal hydrotreater operation, the second-stage $\mathrm{SOH}$ oils (the in-line hydrotreater product oils) are lower in aromatic $\mathrm{H}$ content and higher in paraffinic $\mathrm{H}$ content than the first-stage SOH oils. HTI indicated that the HTU was not operating at optimum performance during Run ALC-1, apparently because a distillation system upset early in the run caused some higher-boiling material to enter the HTU, decreasing the activity of the catalyst. Although HTI observed that the in-line hydrotreater was less effective than expected in removing nitrogen and sulfur from the second-stage SOH, CONSOL data (Table 3) show that the aromatic, hydroaromatic, and paraffinic hydrogen contents of these samples were generally similar to those produced in the coal-only operations during Run CMSL-9. ${ }^{6}$ The Run ALC-1 product (secondstage) SOHs also were less aromatic (more hydrogenated) than those produced in the coal-only operations during Run CMSL-11. ${ }^{7}$ These observations suggest that the hydrogenation effectiveness of the catalyst was minimally affected by the event early in Run ALC-1 that seriously decreased the $\mathrm{N}$ and $\mathrm{S}$ heteroatom removal ability of the HTU catalyst.

The hydrotreated product (second stage SOH) oils from HTI Run ALC-1 were devoid of phenolic $-\mathrm{OH}$, in spite of high phenolic $-\mathrm{OH}$ concentration in the unhydrotreated first-stage $\mathrm{SOH}$ oils and of higher-than-normal concentrations of nitrogen and sulfur in the hydrotreated oil. The phenol removal activity, like the hydrogenation activity, evidently was not significantly impaired by the event that impaired the nitrogen and sulfur removal activity of the catalyst. The high phenolic $-\mathrm{OH}$ concentration of the unhydrotreated first stage $\mathrm{SOH}$ oil indicates that it contains a high concentration (perhaps $25 \%$ ) of phenolic compounds, and is a potential source of phenolic chemicals.

\section{ELEMENTAL ANALYSIS OF THE SOH WATER}

Separator overhead (SOH) water samples from Stage 1 and Stage 2 were analyzed for $\mathrm{pH}$ and weight percent $\mathrm{C}, \mathrm{N}$, and $\mathrm{S}$ (Table 7) for HTI's elemental balances. Relative to the Stage 1 samples, the Stage 2 samples had higher $\mathrm{pH}$ and $\mathrm{S}$ and $\mathrm{N}$ concentrations, and lower $\mathrm{C}$ concentration. At the SOH water product flow rates in Run ALC-1 (about $14 \mathrm{~kg} /$ day), the carbon 
content in the water is about $210 \mathrm{~g} /$ day (depending on the production split between the two stages). At the coal feed rate of Run ALC-1 (about $26 \mathrm{~kg} /$ day), the coal carbon feed rate is about $16.6 \mathrm{~kg} /$ day. Based on this analysis, about $1 \mathrm{wt} \%$ of the coal carbon and somewhat greater

proportions of the coal sulfur and nitrogen reported to the $\mathrm{SOH}$ water. It appears that any error resulting from omitting this stream from elemental balance calculations should be small.

The phenolic compounds in one SOH water sample (Period 13B, first stage) were qualitatively characterized by GC/MS analyses. The procedure used was acidification of the sample with $\mathrm{HCl}$ from a pH of about 9 to a pH of about 1 and extraction of the tar acids with methylene chloride. The appearance of the liquid changed from clear, bright yellow to colorless and slightly turbid upon acidification. Odors detected during acidification (in chronological order) included $\mathrm{H}_{2} \mathrm{~S}, \mathrm{SO}_{2}$, and phenols. Only phenol and the cresols were identified by GC/MS analysis of the tar acids.

\section{FEED SLURRY CHARACTERISTICS}

There had been concern that significant amounts of light oils were recycled during Run ALC-1, due to inefficient distillation and other operating conditions. To address this concern, feed slurry samples from each condition of Run ALC-1 were distilled to $343^{\circ} \mathrm{C}\left(650^{\circ} \mathrm{F}\right)$ and $524^{\circ} \mathrm{C}$ $\left(975^{\circ} \mathrm{F}\right)$. The resid was extracted with tetrahydrofuran, and ashed to determine other components as shown in Table 8 . An important result from this characterization is that only a small quantity of $343^{\circ} \mathrm{C}^{-}\left(650^{\circ} \mathrm{F}^{-}\right)$material was recycled in Run ALC-1 ( $\leq 5 \%$ of feed slurry). This presumes that no light material was lost before the samples were shipped to CONSOL.

CONSOL data (Figure 1, Tables 2 and 8 ) indicate that the feed slurry during Conditions 2-4 (Periods 13,17 and 20 ) contained about twice as much THF-soluble $524^{\circ} \mathrm{C}^{+}\left(975^{\circ} \mathrm{F}^{+}\right)$resid as the feed slurry from Conditions 1 and 5 (Periods 6 and 25). The resid content of the Period 5 feed slurry was very high, for no apparent reason. Perhaps the higher concentration of higherboiling material in the feed slurry contributed to the lower distillate yields obtained when oilagglomerated coal was fed. Three factors may relate to the higher resid concentration in the feed slurry when oil-agglomerated coal was fed in Conditions 2-4. First, the higher coal conversion obtained when agglomerates were fed could result in a higher concentration of resid in the recycle streams. Second, removal of an amount of distillate roughly equivalent to that fed with the agglomerated coal may have left only the higher-boiling material for recycle. In fact, the recycle strategy for the entire run was to take the lowest-boiling material as product; perhaps this degree 
of selectivity only became available with operating-condition changes that came into effect when oil-agglomerated coal was fed. The third possible factor is more efficient extraction of soluble resid from the PFC when oil agglomerates were fed (discussed below). This would increase the availability of soluble resid for recycle, although the quantitative effect on feed slurry composition was not considered. The recycle stream composition was controlled by the total recycle rate and solids recycle rate; soluble-resid recycle rate was not directly controlled by the plant operators.

\section{CONCENTRATIONS OF MO AND Fe IN FEED AND PRODUCT STREAMS}

$\mathrm{Mo}$ and $\mathrm{Fe}$ concentrations in the second-stage pressure-filter cake (PFC) samples from Run ALC-1 were determined. They are presented in Table 9 on three bases: (1) metal oxide as weight percent of the $\mathrm{SO}_{3}$-containing ash; (2) element as a weight percent of the $\mathrm{SO}_{3}$-free ash; and (3) element as a weight percent of the $\mathrm{SO}_{3}$-containing ash. The CONSOL Mo concentrations agree with those reported by HTI for the second-stage pressure-filter cake. However, many of CONSOL's Fe concentrations are $20 \%$ (or more) higher than those reported by HTI.

Mo and $\mathrm{Fe}$ concentrations were determined in the $524^{\circ} \mathrm{C}^{+}\left(975^{\circ} \mathrm{F}^{+}\right)$resids from all of the Run ALC-1 feed slurry samples (Table 10). The data provide a direct measurement of the total $\mathrm{Mo}$ and $\mathrm{Fe}$ concentrations in the reactor feed. The concentrations of the metals in the ash agree well with the pressure filter cake (PFC) results reported in Table 9 . Elemental Mo concentrations for the entire set (PFC and feed slurry) ranged from 0.19 to $0.47 \mathrm{wt} \%$ of the $\mathrm{SO}_{3}$-free ash, and approximately doubled when agglomerated coal was fed (partly in response to the reduced ash content of the agglomerated coal). Fe concentrations for the entire set ranged from 17.9 to $28.1 \mathrm{wt} \%$ of the $\mathrm{SO}_{3}$-free ash, and decreased from Condition 2 through the end of the run.

The Mo and Fe concentrations were combined with HTI material balance data to calculate Mo and Fe balances and to determine the apparent Mo and Fe catalyst addition rates. Intermediate and final calculated results are shown in Table 11. The following assumptions were used in the calculations: raw coal $\mathrm{SO}_{3}$-free ash content of $5.50 \% \mathrm{MF}$; agglomerated coal $\mathrm{SO}_{3}$ free ash content of $3.30 \% \mathrm{MF}$; added $\mathrm{Fe}_{2} \mathrm{O}_{3}$ was $1.4 \%$ or $0.98 \%$ of the MF coal rate (for calculating total ash fed to system); Mo in the raw or agglomerated coal was ignored (the MF raw or agglomerated coal contains ca. 2 ppm Mo); the $\mathrm{Fe}_{2} \mathrm{O}_{3}$ content of the raw coal $\mathrm{SO}_{3}$-free ash was 6.66\%; and the $\mathrm{Fe}_{2} \mathrm{O}_{3}$ content of the agglomerated coal $\mathrm{SO}_{3}$-free ash was $9.57 \%$. The data on which the calculations are based were obtained from this report and other reports. ${ }^{1,4-5}$ 
The Mo and $\mathrm{Fe}$ balances were calculated for each test period shown as the ratio of total grams of Mo or Fe out to the total grams of Mo or $\mathrm{Fe}$ in. Total Mo or $\mathrm{Fe}$ in was obtained by multiplying the weight percent $\mathrm{Mo}$ or $\mathrm{Fe}$ in the feed slurry $\mathrm{SO}_{3}$-free ash by the grams of ash fed in that period in the fresh coal and fresh iron catalyst, and in the pressure-filter cake (PFC) recycle. The amount of ash fed in the fresh coal was obtained from the amount of MF coal fed (HTI material balance data) and the $\mathrm{SO}_{3}$-free ash content of the raw or agglomerated coal. The ash fed in the added Fe catalyst was obtained as $1.4 \mathrm{wt} \%$ of the amount of MF coal fed (for the $10,000 \mathrm{mg} / \mathrm{kg}$ Fe target addition rate), or as $0.98 \mathrm{wt} \%$ of the amount of MF coal fed (for the $7,000 \mathrm{mg} / \mathrm{kg} \mathrm{Fe}$ target addition rate). The ash fed in the recycle PFC was obtained from HTl's recycle ratio of insoluble organic matter (IOM) plus $\mathrm{SO}_{3}$-free ash to MF coal, and $\mathrm{HTI}$ 's measurements of IOM and $\mathrm{SO}_{3}$-free ash contents of the PFC. The total amount of Mo or Fe out was obtained by multiplying the wt \% Mo or Fe in the PFC by the grams of total PFC produced for the period (HTI material balance data).

Apparent catalyst addition rates were obtained by subtracting the amount of $\mathrm{Mo}$ or $\mathrm{Fe}$ in the recycled $\mathrm{PFC}$ from the total amount of Mo or Fe fed in the feed slurry. The rates were calculated on an MF coal basis. In the Fe case, the coal Fe contribution was backed out, so that the results represent only added $\mathrm{Fe}$. The ca. $2 \mathrm{mg} / \mathrm{kg} \mathrm{Mo} \mathrm{in} \mathrm{the} \mathrm{raw} \mathrm{coal} \mathrm{was} \mathrm{not} \mathrm{backed} \mathrm{out.}$

The resulting Mo balance closures were $80-119 \%$, and the Fe balance closures were $98-120 \%$. The balances were generally good; that is, there was not a consistent or gross imbalance between feed and product rates. These balances generally verify the validity and consistency of the analytical measurements. The calculated apparent Mo catalyst addition rates (MF coal basis) were 110-269 mg/kg Mo when the target Mo concentration was $100 \mathrm{mg} / \mathrm{kg}$, and $94-161 \mathrm{mg} / \mathrm{kg}$ when the target rate was $70 \mathrm{mg} / \mathrm{kg} \mathrm{Mo}$. The calculated apparent Fe catalyst addition rates (MF coal basis) were 3,928-13,642 $\mathrm{mg} / \mathrm{kg}$ Fe when the target $\mathrm{Fe}$ concentration was $10,000 \mathrm{mg} / \mathrm{kg}$, and $7,309-8,571 \mathrm{mg} / \mathrm{kg}$ when the target rate was $7,000 \mathrm{mg} / \mathrm{kg} \mathrm{Mo}$.

These results indicate that the Mo addition rate was $10-169 \%$ in excess of the target rate. The excess amount seemed to be largest during periods when agglomerated coal was fed. The generally good Mo balance suggests that the Mo addition rate was inadvertently high, rather than being caused by introduction of Mo from a spurious source. Any spurious source of Mo would have to introduce a high Mo concentration in the feed slurry to be consistent with these results. 
The results show that the Fe added in Period 13 (Condition 2) was only $39 \%$ of the target rate, but in the other periods was $4-36 \%$ in excess of the target rate.

\section{EFFECT ON PROCESS STREAM PROPERTIES OF FEEDING COAL CLEANED BY OIL AGGLOMERATION AT LOW $\mathrm{pH}$}

The ash content of the $0-6$ bottoms stream was lower when agglomerates were fed in Conditions 2-4, Periods 13, 17, and 20 (9.3-9.8\%, vs. $11.3-12.7 \%$ in Conditions 1 and 5). A similar but less pronounced pattern was observed in the feed slurry stream $(6.9-7.8 \%$ vs. 8.2 $8.9 \%$ in Conditions 1 and 5). The lower ash concentrations in the samples seem to be related to the lower ash content of the agglomerated feed coal, but the relationship is not completely straightforward, especially for the 0-6 bottoms samples. Although the feed coal ash content was lower in the oil-agglomerates, the addition rate of Fe-based catalyst was changed, which contributes significantly to the ash content of the total feed. There is also a significant rate of ash recycle, which is larger than the feed rate of ash from the fresh coal. The ash recycle rate was held roughly constant, although changes that result from feeding agglomerates (such as an increase in coal conversion) would be expected to affect the recycle ash rate.

Extraction of THF-soluble $524^{\circ} \mathrm{C}^{+}\left(975^{\circ} \mathrm{F}^{+}\right)$resid from the PFCs was more complete when oilagglomerated coal was fed in Conditions 2-4 (Periods 13,17 , and 20 , Figure 3 ). When raw coal was fed (Periods 6 and 25), 9-34\% solubles remained in the toluene-extracted PFCs. When oilagglomerated coal was fed, only $5 \%$ or less THF-solubles remained in the toluene-extracted PFCs. HTI's data for hot-quinoline solubles confirm this effect. ${ }^{1}$ Perhaps the improvement in extraction efficiency was related to the improved filtration observed when agglomerates were fed. ${ }^{8}$ If it can be demonstrated that oil agglomeration at low $\mathrm{pH}$ improves liquefaction plant operability, low pH agglomeration would have a significant economic impact. The apparently higher Mo addition rate during these periods may have contributed to the benefits of feeding oilagglomerated coal.

\section{EFFECT OF THE DECREASE IN NOMINAL FRESH CATALYST ADDITION RATE}

Decreasing the nominal fresh catalyst addition rate after Condition 2 had no detrimental effect on process sample characteristics. In fact, performance improved (as manifested by increased light distillate yield and decreased resid yield). As noted in a prior section, the concentrations of Mo and $\mathrm{Fe}$ in feed slurry and PFC samples suggest that the actual catalyst feed rates differed 
considerably from the nominal rates in some cases. Those results (Table 11) indicate that the Fe addition rate approximately doubled from Condition 2 to Condition 3, whereas the Mo addition rate decreased by $60 \%$ (but remained much higher than the target rate of $70 \mathrm{mg} / \mathrm{kg}$ ). Perhaps the increased $\mathrm{Fe}$ rate contributed to improved performance during Condition 3 . No operating condition obviously changed sufficiently to account for the improved performance, although changes in distillation configuration and distillation conditions could have some effect on performance that is difficult to account for.

\section{EFFECT OF RECYCLE SOLVENT DEWAXING AND HYDROGENATION}

Vacuum distillates from Run ALC-1 were dewaxed in the laboratory and the dewaxing feeds and fractions were analyzed. Microautoclave solvent quality tests and ${ }^{1} \mathrm{H}-\mathrm{NMR}$ spectroscopy were used) to determine the effectiveness of the Run ALC-1 dewaxing and hydrotreating operations. Either vacuum still overhead (VSOH) samples or the laboratory-generated $454^{\circ} \mathrm{C}^{-}\left(850^{\circ} \mathrm{F}^{-}\right)$ distillate of $0-6$ bottoms samples were tested.

Table 12 shows that the 0-6 bottoms distillate contained about 5-6\% wax before dewaxing operations were started (Period 20). During dewaxing operations (Period 25), the wax content of the second-stage heavy distillate (VSOH) product was reduced to 1-2\%, presumably mostly freshly produced wax. The heavy distillate (VSOH, dewaxed), also taken from period 25 but downstream of dewaxing, was virtually devoid of wax.

The ${ }^{1} \mathrm{H}-\mathrm{NMR}$ proton distributions and microautoclave solvent quality test results for heavy distillates relevant to the dewaxing operations are shown in Tables 3 and 6 . The data are summarized in Table 13; averages and standard deviations are shown where possible. The data in Table 13 show that the paraffinic nature of the heavy distillate was reduced by dewaxing and there was a minor improvement in solvent quality, as measured by two types of microautoclave liquefaction tests. Hydrotreating was effective in producing hydroaromatics and in improving solvent quality.

For reasons not clear, the VSOH and 0-6 bottoms distillates from periods 13, 17, and 20 were more phenolic than the corresponding untreated period 25 samples (Table 13, $0.98 \mathrm{meq} / \mathrm{g}$ vs. $0.54 \mathrm{meq} / \mathrm{g}$ ). Dewaxing had no impact on phenolic $-\mathrm{OH}$ concentration, but hydrotreating virtually eliminated the phenols $(0.07 \mathrm{meq} / \mathrm{g}$ found in hydrotreated samples). 
In summary, the results indicate that the bench-unit dewaxing operations were effective. However, the low concentration of wax in the feed limited the impact of the dewaxing on improving solvent quality. The solvent hydrotreating operation increased the hydroaromatic hydrogen content of the distillate and improved its solvent quality.

\section{WAX YIELDS AND CHARACTERISTICS}

The wax produced during Run ALC-1 period 25 was characterized by elemental, ${ }^{1} \mathrm{H}-\mathrm{NMR}$, and GC/MS analyses (Table 14). The wax was black and had a strong odor. The elemental analyses indicate that it contained over $13 \%$ hydrogen; however, four replicate elemental analyses gave poor repeatability (about $5 \%$ relative). It appears that the reported carbon content $(74 \mathrm{wt} \%)$ is incorrectly low, because the ${ }^{1} \mathrm{H}-\mathrm{NMR}$ and GC/MS analyses did not indicate major concentrations of any components except alkanes. The wax was de-oiled via CONSOL's standard dewaxing procedure at $-5{ }^{\circ} \mathrm{C}$ in acetone to indicate wax purity. This yielded $75 \%$ de-oiled wax, but also $2 \%$ THF insolubles (Table 14). The de-oiled wax has less than $1 \%$ aromatic protons (Table 14). 
RECALIBRATION OF FTIR SPECTROSCOPIC METHOD FOR PHENOLIC -OH DETERMINATION

The Fourier-transform infrared spectroscopic (FTIR) method used to determine phenolic - $\mathrm{OH}$ concentration in liquefaction samples was recalibrated and implemented on a new FTIR instrument. A calibration was obtained from spectra of six standard phenol compounds. An error estimate for each determination was added to CONSOL's analysis procedure software. The method was validated by comparison of results from samples run on both the old and new systems. A detailed discussion is presented with the results in Appendix 1. 


\section{CRUDE OIL ASSAYS OF NET PRODUCTS OF HTI RUN PB-03}

CONSOL arranged to have crude oil assays conducted on the net products of HTI Run PB-03, at DOE's request. Crude oil assays were conducted on net products obtained during periods of Run PB-03 with and without use of the on-line hydrotreater.

$\mathrm{HTI}$ provided CONSOL with fifteen individual separator overhead (SOH) samples from HTI Run PB-03. The samples were contained in 1 gt $(0.95 \mathrm{~L})$ plastic bottles. CONSOL prepared and blended these materials to produce two large samples for crude oil assay testing. The blended samples represent periods with (Periods 6, 7, and 8 ) and without (Periods 9, 10, and 11) the online hydrotreater in operation. Most of the eight individual samples of hydrotreated oil contained a water layer and a sediment (or emulsion) at the oil/water interface. The blends consisted of only the sediment-free oil phase, which was obtained by decanting the easily separated portion of the oil phase, then passing the remainder of the decant through a filter to retain any water or sediment. The water, sediment, and a small remaining oil layer were discarded. Although there was no visual evidence of water or sediment in the seven individual unhydrotreated samples, their dark color made it difficult to ascertain this; therefore, the same procedure was used for blending them. The blended materials weighed $19.96 \mathrm{~kg}$ and $17.64 \mathrm{~kg}$, respectively. Each of the two blended materials was placed in two steel 5 gal $(3.79 \mathrm{~L})$ cans and shipped to Inchcape Testing Services Caleb Brett Laboratory in Houston, TX. Caleb Brett's reports on the crude oil assays appear as Appendix 3.

Caleb Brett returned all remaining samples and fractions of the two crudes to CONSOL after completion of the crude oil assays. One of the crudes and several of the fractions could not be returned to CONSOL because they were consumed during the crude oil assays. CONSOL determined the proton distributions by ${ }^{1} \mathrm{H}-\mathrm{NMR}$ and the phenolic $-\mathrm{OH}$ concentrations by FTIR on the returned materials. Those results appear in Table 15. 


\section{RESID REACTIVITY STUDIES}

Knowledge of the chemistry of resids and understanding of resid reactivity is key to improving direct liquefaction process design and process economics. One approach to acquiring this knowledge is to correlate the chemical characteristics of the resids with the process conditions under which the resids were produced. This was examined in CONSOL's Contract DE-AC2289PC89883. ${ }^{12}$ Another approach is to correlate the relative reactivities of the resids with their chemical characteristics. In order to acquire reactivity data on coal liquefaction resids, a standard empirical test for resid reactivity was developed by the University of Delaware (UOD) under subcontract to CONSOL. Distillation resids chosen by CONSOL for their representation of many different process conditions are being tested (Appendix 4).

To further study the reactivity of resids and their correlation with chemical characteristics, two models are being developed by the University of Delaware: a structural representation model and a reactivity model. Both models can be used for predictive purposes. Analytical data acquired by CONSOL and UOD on the resids being examined by UOD will be incorporated into the structural model. Conversion data and reaction product characteristics will be used to train the reactivity model. However, the University of Delaware resid reactivity tests are being carried out in a short time batch reactor (STBR) with very rapid heating and cooling times. All products of reaction are consumed in the resid conversion determinations. Therefore, there are insufficient reaction products to obtain chemical characterization of them for development of the reactivity model. Additionally, the product gases from the STBR are not analyzed. In order too produce sufficient sample for analysis of products (including gases), a series of resid reactivity tests were made in $45 \mathrm{~mL}$ microautoclaves.

These resid reactivity tests were carried out at CONSOL on the set of fifteen resids that currently are under study at the University of Delaware. Temperature and residence time were the same as those used in the STBR: $420^{\circ} \mathrm{C}$ for $30 \mathrm{~min}$ residence time. The reactor charge in all tests was $4.00 \mathrm{~g}$ resid, $8.00 \mathrm{~g}$ tetralin, and $10.3 \mathrm{MPa}(1500 \mathrm{psi})$ (cold) $\mathrm{H}_{2}$. Each resid was reacted with and without molybdenum naphthenate catalyst precursor. In tests with molybdenum naphthenate, a charge of methyldisulfide (also known as dimethyldisulfide, DMDS) was added to sulfide the catalyst precursor. The molybdenum naphthenate charge, when used, was $2.00 \mathrm{~g}$ ( $3 \mathrm{wt} \% \mathrm{Mo}$ on resid); each catalytic test used $0.5 \mathrm{~g}$ of DMDS. A full description of experimental methods is given in the Experimental section (Section 4). 
Product analyses included gas chromatography of the collected gases, distillation of the condensed products to an atmospheric equivalent temperature of $454^{\circ} \mathrm{C}\left(850^{\circ} \mathrm{F}\right)$, and elemental analysis of the $454^{\circ} \mathrm{C}^{+}\left(850^{\circ} \mathrm{F}^{+}\right)$product fraction. Product distributions are presented in Table 16. The table gives the feed, product distribution, and resid conversion on an $\mathrm{SO}_{3}$-free ash-free basis. The analyses of the $454^{\circ} \mathrm{C}^{+}\left(850^{\circ} \mathrm{F}^{+}\right)$fraction of the products also are provided on that basis. Gas chromatography data were obtained on a volume percent basis. From the vent gas mass and the volume percent of each component, a gram quantity is determined; the gram quantities are provided in Table 16. The nitrogen and oxygen concentrations are presumed to be due to air introduced during charging, sampling collection, and sample analysis; they are normalized out of the analyses. A blank space in Table 16 under Analysis of Product Gases indicates that GC analysis was not performed for that sample.

There was $<<1$ vol \% methane and $\mathrm{H}_{2} \mathrm{~S}$ detected in the non-catalytic tests. In catalyzed tests, hydrogen reacts with the DMDS to produce methane and $\mathrm{H}_{2} \mathrm{~S}$.

Many of the tests were repeated; these replicates are represented on Table 16 by a postscript letter (e.g., 22a means this was the second test at the same conditions of test 22 , or $14 \mathrm{c}$ means that this is the fourth test at the same conditions as test 14). All analyses were sent to UOD for inclusion in the structural model.

A comparison to the resid conversion values obtained at Delaware (Table 17) shows that the microreactor gave lower conversions than the STBR. The slow heat-up of the microreactor or other scaling factors may be responsible for the difference. Because of the differences in conversion values obtained with the two reaction systems, the microautoclave data were not included in UOD's modeling of resid conversion. The model could conceivably be reworked to account for different reactor systems and accept these data; however, there is no current plan to do this. 


\section{Section 4 \\ EXPERIMENTAL}

\section{RESID REACTIVITY TESTS}

The sequence for resid reactivity tests was: The total charge for all tests was $4.00 \mathrm{~g}$ resid, $8.00 \mathrm{~g}$ tetralin, and 10.3 MPa (1500 psi) (cold) $\mathrm{H}_{2}$. In tests with molybdenum naphthenate, a charge of methyldisulfide (also known as dimethyldisulfide, DMDS) was added to sulfide the catalyst precursor. The molybdenum naphthenate charge, when used, was $2.00 \mathrm{~g} \mathrm{(} 3 \mathrm{wt} \%$ Mo on resid); each catalytic test used $0.5 \mathrm{~g}$ of DMDS. After sealing, the vessel was connected to the shaker and shaking was started. While shaking, the microautoclave was lowered into a preheated sand bath. Reaction temperatures were achieved in two to four min. Reaction temperature for all tests was $420^{\circ} \mathrm{C}$. At the end of the $30 \mathrm{~min}$ residence time, the microautoclave was removed from the sand bath and immersed in ambient-temperature water while still shaking. The contents were quenched to ca. $100^{\circ} \mathrm{C}$ in less than $90 \mathrm{sec}$. The $30 \mathrm{~min}$ reaction time includes the time from immersion of the microautoclave in the sand bath until immersion in the water bath.

A post-run weight was obtained to check for leaks. The microautoclave was attached to a pressure gauge at the fitting above the fill valve. It then was submersed in a dry ice/acetone bath until the internal thermocouple read $-70^{\circ} \mathrm{C}$. The valve was opened and a cold temperature gas pressure reading was obtained. The microautoclave was removed from the dry ice/acetone bath and allowed to slowly warm to room temperature; pressure was continually monitored as the vessel warmed. When the internal thermocouple registered room temperature $\left(20-22^{\circ} \mathrm{C}\right)$, a final pressure reading was recorded. The gas was vented into a one-liter evacuated chamber; samples were taken from this chamber for gas chromatography analyses. Gas chromatography was performed using a four-column Carle Model III analytical gas chromatograph. A calibrated standard gas mixture (Table 18) was used to calculate gas composition. The remaining gas was vented. The microautoclave and contents were reweighed. Gas make was determined by the difference in the weight before the tests and after venting.

Prior to distillation of the sample to $454^{\circ} \mathrm{C}\left(850^{\circ} \mathrm{F}\right)$, the microautoclave head was removed. $\mathrm{A}$ distillation head was attached. The microautoclave body was encased in a custom-designed heating mantle. The microautoclave was heated to a temperature of $265^{\circ} \mathrm{C}\left(510^{\circ} \mathrm{F}\right)$ at 5 torr $\left(454^{\circ} \mathrm{C}\left(850^{\circ} \mathrm{F}\right)\right.$ atmospheric equivalent temperature). The distillate was retained for further 
analysis. The $454^{\circ} \mathrm{C}^{+}\left(850^{\circ} \mathrm{F}^{+}\right)$contents of the microautoclave were submitted for elemental analysis.

Resid conversion was determined on an $\mathrm{SO}_{3}$-free, ash-free basis as follows:

$$
\text { resid conversion }=100-\frac{\text { filter cake }-\mathrm{SO}_{3} \text {-free resid ash }}{\mathrm{MAF} \text { resid }} \times 100
$$

\section{OTHER TESTS}

The experimental procedures used to produce results presented in this report have been described previously. ${ }^{9-11}$ 


\section{Section 5}

\section{REFERENCES}

1. Hu, J. L.; Lee, L. K. Section Two of "Advanced Direct Liquefaction Concepts for PETC Generic Units, Phase II, Quarterly Technical Progress Report for Period July through September, 1996", DOE/PC/91040-72, November 1996 (Draft).

2. Robbins, G. A.; Winschel, R. A.; Burke, F. P.; Derbyshire, F. J.; J.; Givens, E. N.; Hu, J.; Lee, T. L. K.; Miller, J. E.; Stephens, H. P.; and Peluso, M. "Testing of Advanced Liquefaction Concepts in HTI Run ALC-1: Coal Cleaning and Recycle Solvent Treatment", Proceedings of the U.S.IJapan Joint Technical Workshop. State College, PA, September 30-October 2, 1996, U.S. DOE.

3. Robbins, G. A.; Winschel, R. A.; Burke, F. P.; Derbyshire, F. J.; Givens, E. N.; Hu, J.; Lee, T. L. K.; Miller, J. E.; Stephens, H. P.; and Peluso, M. "Testing of Advanced Liquefaction Concepts in HTI Run ALC-1: Coal Cleaning and Recycle Solvent Treatment", Proceedings of the First Joint Power and Fuel Systems Contractors Conference, Pittsburgh, PA, July 9-11, 1996, U.S. DOE/PETC.

4. Winschel, R. A.; Robbins, G. A., Quarterly Report on Subcontract UKRF-4-25582-92-76 to CONSOL Inc. under DOE contract No. DE-AC22-91PC91040 "Advanced Coal Liquefaction Concepts for the PETC Generic Bench-Scale Unit", Report period April 1-June 30, 1996, January 16, 1997.

5. Winschel, R. A.; Robbins, G. A., Quarterly Report on Subcontract UKRF-4-25582-92-76 to CONSOL Inc. under DOE contract No. DE-AC22-91PC91040, "Advanced Coal Liquefaction Concepts for the PETC Generic Bench-Scale Unit", Report period July 1September 30, 1996, February 6, 1997.

6. Robbins, G. A.; Brandes, S. D.; Winschel, R. A.; Burke, F. P. "A Characterization and Evaluation of Coal Liquefaction Process Streams, Quarterly Technical Progress Report October 1 through December 31, 1995", DOE/PC 93054-25, May 1996.

7. Robbins, G. A.; Brandes, S. D.; Winschel, R. A.; Burke, F. P. "A Characterization and Evaluation of Coal Liquefaction Process Streams, Quarterly Technical Progress Report, April 1 through June 30, 1996", DOE/PC 93054-34, March 1997.

8. J. Hu (HTI), personal communication with G. Robbins (CONSOL).

9. Robbins, G. A.; Brandes, S. D.; Winschel, R. A.; Burke, F. P. "A Characterization and Evaluation of Coal Liquefaction Process Streams, Quarterly Technical Progress Report January 1 through March 31, 1996", DOE/PC 93054-29, July 1996.

10. Burke, F. P.; Winschel, R. A.; Robbins, G. A. "Recycle Slurry Oil Characterization, Final Report, October 1980 through March 1985", DOE/PC 30027-61, March 1985. 
11. Winschel, R. A.; Robbins, G. A.; Burke, F. P. "Coal Liquefaction Process Solvent Characterization and Evaluation, Technical Progress Report, July 1985 through September 1985", DOE/PC 70018-13, December 1985.

12. Brandes, S. D.; Robbins, G. A.; Winschel, R. A.; Burke, F. P. "Coal Liquefaction Process Streams Characterization and Evaluation, Volume II, Participants Program Final Summary Evaluation", DOE/PC 89883-93, May 1994. 
TABLE 1

\begin{abstract}
HTI OPERATING CONDITIONS AND PROCESS PERFORMANCE RESULTS FROM RUN ALC-1 (227-94)
\end{abstract}

\begin{tabular}{|c|c|c|c|c|c|c|}
\hline $\begin{array}{l}\text { Date, } 1996 \\
\text { Period } \\
\text { Coal Type } \\
\text { Condition }\end{array}$ & $\begin{array}{c}4 / 24 \\
6 \\
\text { Raw } \\
1\end{array}$ & $\begin{array}{c}4 / 30 \\
12 \\
\text { Agglom } \\
2\end{array}$ & $\begin{array}{c}5 / 1 \\
13 \\
\text { Agglom } \\
2\end{array}$ & $\begin{array}{c}5 / 5 \\
17 \\
\text { Agglom } \\
3\end{array}$ & $\begin{array}{c}5 / 8 \\
20 \\
\text { Agglom } \\
4\end{array}$ & $\begin{array}{c}5 / 13 \\
25 \\
\text { Raw } \\
5\end{array}$ \\
\hline $\begin{array}{l}\text { Net Normalized Yields, wt \% MAF } \\
\text { Coal } \\
\mathrm{C}_{1} \text { in Gases } \\
\mathrm{C}_{2} \text { in Gases } \\
\mathrm{C}_{3} \text { in Gases } \\
\mathrm{C}_{1}-\mathrm{C}_{3} \text { in Gases } \\
\mathrm{C}_{4}-\mathrm{C}_{7} \text { in Gases } \\
\text { IBP-177 }{ }^{\circ} \mathrm{C}\left(\text { (IBP-350 }{ }^{\circ} \mathrm{F}\right) \\
177-260^{\circ} \mathrm{C}\left(350-500^{\circ} \mathrm{F}\right) \\
260-343^{\circ} \mathrm{C}\left(500-650^{\circ} \mathrm{F}\right) \\
343-454^{\circ} \mathrm{C}\left(650-850^{\circ} \mathrm{F}\right) \\
454524^{\circ} \mathrm{C}\left(850-975^{\circ} \mathrm{F}\right) \\
524^{\circ} \mathrm{C}^{+}\left(975^{\circ} \mathrm{F}^{+}\right) \\
\text {Unconverted Coal } \\
\text { Water Yield by Material Balance } \\
\mathrm{COx} \\
\mathrm{NH}_{3} \\
\mathrm{H}_{2} \mathrm{~S} \\
\text { Wax Product }\end{array}$ & $\begin{array}{r}3.26 \\
2.79 \\
3.37 \\
9.42 \\
4.95 \\
13.65 \\
11.29 \\
23.34 \\
14.60 \\
1.21 \\
4.33 \\
5.04 \\
13.83 \\
5.92 \\
0.73 \\
-0.77 \\
-\end{array}$ & $\begin{array}{r}2.98 \\
3.28 \\
5.01 \\
11.27 \\
5.52 \\
13.40 \\
7.71 \\
20.13 \\
13.77 \\
-0.34 \\
12.34 \\
2.16 \\
15.23 \\
4.28 \\
0.55 \\
-0.25 \\
-\end{array}$ & $\begin{array}{r}4.22 \\
3.39 \\
4.31 \\
11.92 \\
5.19 \\
13.86 \\
7.33 \\
19.76 \\
8.86 \\
1.50 \\
15.42 \\
2.31 \\
17.03 \\
2.79 \\
0.53 \\
-0.27 \\
-\end{array}$ & $\begin{array}{r}3.41 \\
3.09 \\
4.34 \\
10.84 \\
5.06 \\
14.55 \\
7.75 \\
23.60 \\
12.87 \\
-2.52 \\
10.38 \\
2.42 \\
16.19 \\
4.44 \\
0.62 \\
0.03 \\
-\end{array}$ & $\begin{array}{r}3.32 \\
3.49 \\
5.59 \\
12.40 \\
6.48 \\
16.53 \\
9.70 \\
23.88 \\
13.24 \\
-3.75 \\
4.56 \\
2.51 \\
16.20 \\
4.27 \\
0.90 \\
-0.09 \\
-\end{array}$ & $\begin{array}{r}2.90(\mathrm{a}) \\
2.90(\mathrm{a}) \\
3.60(\mathrm{a}) \\
9.40(\mathrm{a}) \\
4.17(\mathrm{a}) \\
12.66 \\
13.43 \\
31.11 \\
5.46 \\
-1.01 \\
7.38 \\
5.28 \\
13.74 \\
6.59 \\
1.04 \\
-1.13 \\
4.79\end{array}$ \\
\hline $\begin{array}{l}\text { Process Performance, wt \% MAF } \\
\text { Coal } \\
\text { Hydrogen Consumption } \\
\text { Coal Conversion ( } \mathrm{SO}_{3} \text { free) } \\
524^{\circ} \mathrm{C}^{+} \text {Conversion } \\
\mathrm{C}_{4}-524^{\circ} \mathrm{C} \text { Distillate } \\
524^{\circ} \mathrm{C}^{+} \text {Resid Yield, MAF } \\
\mathrm{C}_{4}-343^{\circ} \mathrm{C} \text { Distillate } \\
343^{\circ} \mathrm{C}^{+} \text {Yield }\end{array}$ & $\begin{array}{r}7.5 \\
95.0 \\
90.6 \\
69.0 \\
4.3 \\
53.2 \\
20.1\end{array}$ & $\begin{array}{r}5.8 \\
97.8 \\
85.5 \\
60.2 \\
12.3 \\
46.8 \\
25.8\end{array}$ & $\begin{array}{r}6.2 \\
97.7 \\
82.3 \\
56.5 \\
15.4 \\
46.1 \\
25.8\end{array}$ & $\begin{array}{r}6.2 \\
97.6 \\
87.2 \\
61.3 \\
10.4 \\
51.0 \\
20.7\end{array}$ & $\begin{array}{r}6.8 \\
97.5 \\
92.9 \\
66.1 \\
4.6 \\
56.6 \\
14.1\end{array}$ & $\begin{array}{r}8.1 \\
94.7 \\
87.5 \\
70.6(\mathrm{~b}) \\
7.4 \\
66.2(\mathrm{~b}) \\
11.8\end{array}$ \\
\hline 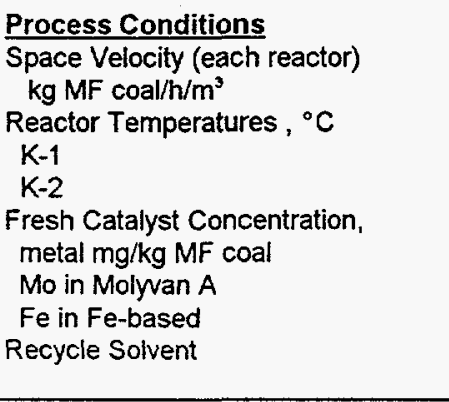 & $\begin{array}{r}675 \\
442 \\
453 \\
\\
100 \\
10000 \\
\text { PFLIPFC }\end{array}$ & $\begin{array}{r}527 \\
443 \\
453 \\
\\
101 \\
10000 \\
\text { PFCNSBI } \\
\text { VSOH } \\
\end{array}$ & $\begin{array}{r}558 \\
442 \\
453 \\
\\
100 \\
9870 \\
\text { PFCNSB/ } \\
\text { VSOH } \\
\end{array}$ & $\begin{array}{r}550 \\
443 \\
452 \\
\\
71 \\
7120 \\
\text { PFCIVSBI } \\
\text { VSOH } \\
\end{array}$ & $\begin{array}{r}418 \\
443 \\
452 \\
\\
68 \\
6810 \\
\text { PFCNSBI } \\
\text { VSOH } \\
\end{array}$ & $\begin{array}{r}466 \\
443 \\
454 \\
\\
70 \\
6980 \\
\text { PFCNSBI } \\
\text { DWHT-VSOH (c) } \\
\end{array}$ \\
\hline
\end{tabular}

\title{
Other Conditions:
}

1.60 Recycle to MF coal ratio (lowered slightly by wax yield in Condition 5 ).

0.20 Recycle solids to MF coal ratio.

$15 \mathrm{MPa}$ system pressure

\section{Notes:}

(a) Not including small quantities produced in DW-VSOH hydrotreating.

(b) Includes $4.79 \%$ wax product in $343-524^{\circ} \mathrm{C}$ range.

(c) VSOH dewaxed and then hydrotreated (DWHT) prior to recycle. 
TABLE 2

COMPONENT DISTRIBUTION OF WHOLE SAMPLES HTI Run ALC-1

\begin{tabular}{|c|c|c|c|c|c|c|}
\hline \multirow[b]{2}{*}{ Sample Type } & \multirow[b]{2}{*}{ Period } & \multicolumn{5}{|c|}{ Component wt $\%$ of Sample } \\
\hline & & $\begin{array}{l}454^{\circ} \mathrm{C}^{-} \\
\left(850^{\circ} \mathrm{F}-\right) \\
\text { Distillate }\end{array}$ & $\begin{array}{l}\text { THF- } \\
\text { Soluble } \\
\text { Resid }\end{array}$ & IOM & Ash & Total \\
\hline $\begin{array}{l}\text { O-6 Bottoms } \\
\text { O-6 Bottoms } \\
\text { O-6 Bottoms } \\
\text { O-6 Bottoms } \\
\text { O-6 Bottoms }\end{array}$ & $\begin{array}{r}6 \mathrm{~B} \\
13 \mathrm{~B} \\
17 \mathrm{~B} \\
20 \mathrm{~B} \\
25 \mathrm{~B}\end{array}$ & $\begin{array}{l}46.4 \\
53.2 \\
50.2 \\
46.8 \\
46.6\end{array}$ & $\begin{array}{l}36.0 \\
32.1 \\
34.0 \\
34.7 \\
33.4\end{array}$ & $\begin{array}{l}5.0 \\
4.9 \\
5.6 \\
5.3 \\
4.6\end{array}$ & $\begin{array}{r}12.7 \\
9.7 \\
9.8 \\
9.3 \\
11.3\end{array}$ & $\begin{array}{r}100.1 \\
99.9 \\
99.6 \\
96.1 \\
95.7\end{array}$ \\
\hline $\begin{array}{l}\text { PFC (Toluene Extracted) } \\
\text { PFC (Toluene Extracted) } \\
\text { PFC } \\
\text { PFC } \\
\text { PFC (Toluene Extracted) } \\
\text { PFC } \\
\text { PFC } \\
\text { PFC } \\
\text { PFC (Toluene Extracted) } \\
\text { PFC } \\
\text { PFC (Toluene Extracted) }\end{array}$ & $\begin{array}{r}6 \mathrm{~B} \\
13 \mathrm{~B} \\
13 \mathrm{~B} \\
13 \mathrm{~A} \\
17 \mathrm{~B} \\
17 \mathrm{~B} \\
17 \mathrm{~A} \\
20 \mathrm{~B} \\
20 \mathrm{~B} \\
25 \mathrm{~B} \\
25 \mathrm{~B} \\
\end{array}$ & $\begin{array}{l}- \\
- \\
- \\
- \\
- \\
- \\
- \\
-\end{array}$ & $\begin{array}{r}9.3(a) \\
3.0(a) \\
34.1(a) \\
39.2(a) \\
1.9(a) \\
35.0(a) \\
33.6(a) \\
24.9(a) \\
5.0(a) \\
42.5(a) \\
33.7(a) \\
\end{array}$ & $\begin{array}{l}23.1 \\
31.1 \\
20.8 \\
19.2 \\
20.8 \\
19.6 \\
21.8 \\
32.6 \\
31.1 \\
16.7 \\
20.7 \\
\end{array}$ & $\begin{array}{l}67.6 \\
65.7 \\
45.1 \\
41.6 \\
77.3 \\
45.4 \\
44.6 \\
42.5 \\
63.9 \\
40.8 \\
45.6 \\
\end{array}$ & $\begin{array}{l}100.0 \\
100.0 \\
100.0 \\
100.0 \\
100.0 \\
100.0 \\
100.0 \\
100.0 \\
100.0 \\
100.0 \\
100.0 \\
\end{array}$ \\
\hline
\end{tabular}

Note:

Feed slurry component concentrations are provided in Table 8.

(a) Obtained by difference. 
TABLE 3

\section{PROTON DISTRIBUTION OF WHOLE SOLIDS-FREE SAMPLES AND DISTILLATE SAMPLES \\ HTI Run ALC-1}

\begin{tabular}{|c|c|c|c|c|c|c|c|c|}
\hline \multirow[b]{2}{*}{ Sample Type } & \multirow[b]{2}{*}{ Period } & \multicolumn{7}{|c|}{ Proton Distribution, \% } \\
\hline & & $\begin{array}{l}\text { Cond } \\
\text { Arom }\end{array}$ & $\begin{array}{l}\text { Uncond } \\
\text { Arom }\end{array}$ & $\begin{array}{l}\text { Cyclic } \\
\text { Alpha }\end{array}$ & $\begin{array}{l}\text { Alkyl } \\
\text { Alpha }\end{array}$ & $\begin{array}{c}\text { Cyclic } \\
\text { Beta }\end{array}$ & $\begin{array}{l}\text { Alkyl } \\
\text { Beta }\end{array}$ & Gamma \\
\hline L-814 Start-Up Oil & $6 \mathrm{~A}$ & 14.0 & 6.6 & 13.3 & 12.5 & 13.5 & 24.0 & 16.2 \\
\hline $\begin{array}{l}\text { SOH First Stage } \\
\text { SOH First Stage } \\
\text { SOH Second Stage } \\
\text { SOH First Stage } \\
\text { SOH Second Stage } \\
\text { SOH Second Stage } \\
\text { SOH First Stage } \\
\text { SOH Second Stage } \\
\text { SOH First Stage } \\
\text { SOH Second Stage }\end{array}$ & $\begin{array}{r}6 \\
13 \\
13 \\
17 \\
17 A \\
17 B \\
20 B \\
20 B \\
25 B \\
25 B\end{array}$ & $\begin{array}{l}5.2 \\
4.9 \\
1.9 \\
4.4 \\
2.7 \\
2.6 \\
4.5 \\
2.6 \\
3.7 \\
3.0\end{array}$ & $\begin{array}{l}7.9 \\
7.2 \\
3.1 \\
7.8 \\
3.7 \\
3.4 \\
8.9 \\
3.5 \\
7.7 \\
3.7\end{array}$ & $\begin{array}{l}9.0 \\
9.5 \\
6.3 \\
7.8 \\
6.6 \\
6.4 \\
8.3 \\
6.6 \\
7.7 \\
6.7\end{array}$ & $\begin{array}{r}10.4 \\
10.3 \\
5.7 \\
9.8 \\
5.3 \\
5.6 \\
10.3 \\
6.2 \\
9.5 \\
6.2\end{array}$ & $\begin{array}{l}17.3 \\
18.6 \\
20.6 \\
17.3 \\
19.3 \\
19.7 \\
16.7 \\
20.8 \\
19.5 \\
20.1\end{array}$ & $\begin{array}{l}25.6 \\
27.2 \\
33.6 \\
27.4 \\
34.2 \\
33.2 \\
28.0 \\
34.7 \\
27.0 \\
31.2\end{array}$ & $\begin{array}{l}24.7 \\
22.2 \\
28.8 \\
25.4 \\
28.2 \\
29.2 \\
23.4 \\
25.5 \\
25.0 \\
29.0\end{array}$ \\
\hline $\begin{array}{l}\text { PFL } \\
\text { VSOH } \\
\text { VSOH } \\
\left.\text { VSOH (IBP } \times 343^{\circ} \mathrm{C}\right) \\
\text { VSOH }\left(343^{\circ} \mathrm{C} \times 399^{\circ} \mathrm{C}\right) \\
\text { VSOH dewaxed, hydrotreated, } 343^{\circ} \mathrm{C}^{+} \\
\text {VSOH dewaxed, hydrotreated, } 343^{\circ} \mathrm{C}^{-} \\
\left.\text {VSOH (IBP } \times 524^{\circ} \mathrm{C}\right) \\
\text { VSOH dewaxed, hydrotreated, } 343^{\circ} \mathrm{C}^{+} \\
\text {VSOH, dewaxed } \\
\text { VSOH, dewaxed, hydrotreated } \\
\text { Wax }\end{array}$ & $\begin{array}{r}6 \\
13 \\
17 \\
20 \\
20 \\
21 \mathrm{~A} \\
21 \mathrm{~A} \\
25 \\
25 \\
25 \\
25 \\
25\end{array}$ & $\begin{array}{r}19.5 \\
12.2 \\
13.1 \\
9.9 \\
15.3 \\
9.0 \\
5.0 \\
17.8 \\
10.8 \\
17.8 \\
8.2 \\
2.5\end{array}$ & $\begin{array}{r}10.5 \\
10.5 \\
10.3 \\
12.4 \\
8.6 \\
6.6 \\
6.7 \\
6.2 \\
6.3 \\
9.8 \\
6.1 \\
1.4\end{array}$ & $\begin{array}{r}14.4 \\
15.5 \\
14.9 \\
15.5 \\
16.3 \\
15.3 \\
12.8 \\
17.8 \\
18.4 \\
17.1 \\
15.8 \\
4.6\end{array}$ & $\begin{array}{r}11.4 \\
12.5 \\
11.5 \\
13.4 \\
10.8 \\
10.5 \\
9.6 \\
11.0 \\
10.6 \\
11.3 \\
9.9 \\
3.2\end{array}$ & $\begin{array}{r}11.2 \\
14.1 \\
13.1 \\
14.3 \\
12.9 \\
19.8 \\
23.6 \\
15.7 \\
19.7 \\
14.5 \\
21.4 \\
8.1\end{array}$ & $\begin{array}{l}20.2 \\
23.9 \\
24.9 \\
23.4 \\
25.9 \\
23.4 \\
25.1 \\
20.8 \\
21.3 \\
18.0 \\
23.1 \\
68.7\end{array}$ & $\begin{array}{l}12.9 \\
11.1 \\
12.3 \\
11.1 \\
10.2 \\
15.3 \\
17.1 \\
10.6 \\
13.0 \\
11.5 \\
15.5 \\
11.5\end{array}$ \\
\hline VSB from $P F L$ & 25 & 29.9 & 11.5 & 18.9 & 10.1 & 11.1 & 10.8 & 7.9 \\
\hline $\begin{array}{l}\text { Feed Slurry }\left(343{ }^{\circ} \mathrm{C}^{-}\right) \\
\text {Feed Slurry }\left(343 \times 524^{\circ} \mathrm{C}^{-}\right) \\
\text {Feed Slurry }\left(343 \times 524^{\circ} \mathrm{C}^{-}\right) \\
\text {Feed Slurry }\left(343 \times 524^{\circ} \mathrm{C}^{-}\right) \\
\text {Feed Slurry }\left(343^{\circ} \mathrm{C}^{-}\right) \\
\text {Feed Slurry }\left(343 \times 524^{\circ} \mathrm{C}^{-}\right) \\
\text {Feed Slurry }\left(343 \times 524^{\circ} \mathrm{C}^{-}\right) \\
\text {Feed Slurry }\left(343^{\circ} \mathrm{C}^{-}\right)\end{array}$ & $\begin{array}{r}6 \\
6 \\
13 \\
17 \\
17 \\
20 \\
25 \\
25\end{array}$ & $\begin{array}{r}10.4 \\
23.1 \\
16.3 \\
15.8 \\
8.9 \\
15.5 \\
8.4 \\
3.7\end{array}$ & $\begin{array}{l}9.7 \\
6.5 \\
5.8 \\
6.5 \\
8.6 \\
7.3 \\
7.2 \\
6.9\end{array}$ & $\begin{array}{l}15.1 \\
17.1 \\
16.1 \\
16.4 \\
14.0 \\
15.2 \\
15.3 \\
11.6\end{array}$ & $\begin{array}{r}14.0 \\
12.1 \\
10.0 \\
9.1 \\
11.3 \\
9.4 \\
10.2 \\
9.9\end{array}$ & $\begin{array}{l}15.7 \\
12.6 \\
13.7 \\
13.0 \\
16.1 \\
13.2 \\
19.8 \\
23.3\end{array}$ & $\begin{array}{l}23.0 \\
20.1 \\
28.0 \\
28.3 \\
27.3 \\
27.2 \\
23.5 \\
26.3\end{array}$ & $\begin{array}{r}12.0 \\
8.5 \\
10.2 \\
10.9 \\
13.7 \\
12.1 \\
15.6 \\
18.4\end{array}$ \\
\hline $\begin{array}{l}\text { O-6 Bottoms }\left(454^{\circ} \mathrm{C}^{-}\right) \\
\text {O-6 Bottoms }\left(454^{\circ} \mathrm{C}^{-}\right) \\
\text {O-6 Bottoms }\left(454^{\circ} \mathrm{C}^{-}\right) \\
\text {O-6 Bottoms }\left(454^{\circ} \mathrm{C}^{-}\right) \\
\text {O-6 Bottoms }\left(454^{\circ} \mathrm{C}^{-}\right)\end{array}$ & $\begin{array}{r}6 \\
13 \\
17 \\
20 \\
25\end{array}$ & $\begin{array}{l}20.6 \\
15.8 \\
15.4 \\
16.0 \\
14.3\end{array}$ & $\begin{array}{r}8.5 \\
9.9 \\
10.1 \\
10.0 \\
9.2\end{array}$ & $\begin{array}{l}18.2 \\
15.8 \\
15.6 \\
16.2 \\
14.8\end{array}$ & $\begin{array}{l}12.5 \\
11.1 \\
10.7 \\
11.2 \\
11.3\end{array}$ & $\begin{array}{l}12.4 \\
12.6 \\
12.5 \\
12.8 \\
15.4\end{array}$ & $\begin{array}{l}19.9 \\
23.8 \\
24.5 \\
23.3 \\
21.2\end{array}$ & $\begin{array}{r}7.9 \\
10.8 \\
11.2 \\
10.5 \\
13.8\end{array}$ \\
\hline
\end{tabular}


TABLE 4

\section{PROTON DISTRIBUTION OF FILTERED WHOLE SOLIDS-CONTAINING SAMPLES AND RESID SAMPLES HTI Run ALC-1}

\begin{tabular}{|c|c|c|c|c|c|c|c|c|}
\hline \multirow[b]{2}{*}{ Sample Type } & \multirow[b]{2}{*}{ Period } & \multicolumn{7}{|c|}{ Proton Distribution, $\%$} \\
\hline & & $\begin{array}{l}\text { Cond } \\
\text { Arom }\end{array}$ & $\begin{array}{c}\text { Uncond } \\
\text { Arom }\end{array}$ & $\begin{array}{l}\text { Cyclic } \\
\text { Alpha }\end{array}$ & $\begin{array}{l}\text { Alkyl } \\
\text { Alpha }\end{array}$ & $\begin{array}{c}\text { Cyclic } \\
\text { Beta }\end{array}$ & $\begin{array}{l}\text { Alkyl } \\
\text { Beta }\end{array}$ & Gamma \\
\hline $\begin{array}{l}\text { Feed Slurry } \\
\text { Feed Slurry } \\
\text { Feed Slurry } \\
\text { Feed Slurry } \\
\text { Feed Slurry } \\
\text { Feed Slurry }\end{array}$ & $\begin{array}{r}5 \\
6 \\
13 \\
17 \\
20 \\
25\end{array}$ & $\begin{array}{l}21.2 \\
23.5 \\
19.6 \\
20.1 \\
18.5 \\
11.0\end{array}$ & $\begin{array}{r}11.3 \\
8.8 \\
5.9 \\
6.4 \\
8.9 \\
8.5\end{array}$ & $\begin{array}{l}14.3 \\
17.4 \\
18.7 \\
18.2 \\
14.7 \\
12.8\end{array}$ & $\begin{array}{r}12.4 \\
11.5 \\
9.7 \\
9.6 \\
9.2 \\
9.5\end{array}$ & $\begin{array}{l}11.0 \\
11.4 \\
13.0 \\
13.3 \\
12.1 \\
16.9\end{array}$ & $\begin{array}{l}18.1 \\
17.8 \\
23.1 \\
23.4 \\
23.6 \\
24.1\end{array}$ & $\begin{array}{r}11.8 \\
9.7 \\
10.1 \\
9.1 \\
13.0 \\
17.2\end{array}$ \\
\hline $\begin{array}{l}\text { First-Stage Slurry } \\
\text { First-Stage Slurry } \\
\text { First-Stage Slurry } \\
\text { First-Stage Slurry }\end{array}$ & $\begin{array}{l}14 \\
17 \\
21 \\
25\end{array}$ & $\begin{array}{l}16.8 \\
19.2 \\
21.0 \\
16.4\end{array}$ & $\begin{array}{l}8.5 \\
7.1 \\
6.7 \\
7.0\end{array}$ & $\begin{array}{l}16.8 \\
18.2 \\
17.8 \\
18.7\end{array}$ & $\begin{array}{l}11.4 \\
10.9 \\
10.5 \\
10.8\end{array}$ & $\begin{array}{l}13.6 \\
13.7 \\
13.3 \\
16.0\end{array}$ & $\begin{array}{l}22.6 \\
21.6 \\
21.5 \\
20.5\end{array}$ & $\begin{array}{r}10.4 \\
9.3 \\
9.2 \\
10.8\end{array}$ \\
\hline $\begin{array}{l}\text { O-6 Bottoms } \\
\text { O-6 Bottoms } \\
\text { O-6 Bottoms } \\
\text { O-6 Bottoms } \\
\text { O-6 Bottoms }\end{array}$ & $\begin{array}{r}6 \\
13 \\
17 \\
20 \\
25\end{array}$ & $\begin{array}{l}21.6 \\
18.1 \\
20.7 \\
21.3 \\
20.0\end{array}$ & $\begin{array}{r}12.5 \\
11.9 \\
8.6 \\
8.6 \\
7.9\end{array}$ & $\begin{array}{l}16.3 \\
16.0 \\
18.7 \\
18.8 \\
18.5\end{array}$ & $\begin{array}{l}11.7 \\
11.2 \\
10.4 \\
10.7 \\
11.2\end{array}$ & $\begin{array}{l}11.3 \\
12.5 \\
12.9 \\
12.7 \\
13.9\end{array}$ & $\begin{array}{l}16.6 \\
19.4 \\
19.4 \\
20.3 \\
18.2\end{array}$ & $\begin{array}{r}10.1 \\
10.9 \\
9.2 \\
7.6 \\
10.2\end{array}$ \\
\hline $\begin{array}{l}\text { PFC (toluene extracted) } \\
\text { PFC } \\
\text { PFC } \\
\text { PFC } \\
\text { PFC } \\
\text { PFC } \\
\text { PFC }\end{array}$ & $\begin{array}{r}6 A \\
13 A \\
13 B \\
17 A \\
17 B \\
20 B \\
25 B\end{array}$ & $\begin{array}{l}27.2 \\
25.0 \\
23.7 \\
23.3 \\
25.5 \\
22.3 \\
21.6\end{array}$ & $\begin{array}{l}5.5 \\
5.7 \\
5.5 \\
5.1 \\
4.5 \\
7.3 \\
7.1\end{array}$ & $\begin{array}{l}15.5 \\
18.4 \\
18.9 \\
20.3 \\
19.8 \\
16.6 \\
15.6\end{array}$ & $\begin{array}{l}10.0 \\
10.8 \\
10.2 \\
11.6 \\
10.0 \\
10.1 \\
10.4\end{array}$ & $\begin{array}{l}12.0 \\
12.8 \\
13.3 \\
13.3 \\
13.4 \\
12.8 \\
13.7\end{array}$ & $\begin{array}{l}16.6 \\
19.0 \\
18.8 \\
17.1 \\
18.6 \\
19.9 \\
18.3\end{array}$ & $\begin{array}{r}13.2 \\
8.5 \\
9.5 \\
9.2 \\
8.1 \\
11.0 \\
13.2\end{array}$ \\
\hline $\begin{array}{l}\text { Feed Slurry }\left(524^{\circ} \mathrm{C}^{+}\right) \\
\text {Feed Slurry }\left(524^{\circ} \mathrm{C}^{+}\right) \\
\text {Feed Slurry }\left(524^{\circ} \mathrm{C}^{+}\right) \\
\text {Feed Slury }\left(524^{\circ} \mathrm{C}^{+}\right) \\
\text {Feed Slurry }\left(524^{\circ} \mathrm{C}^{+}\right)\end{array}$ & $\begin{array}{r}6 \\
13 \\
17 \\
20 \\
25\end{array}$ & $\begin{array}{l}34.1 \\
27.6 \\
26.6 \\
26.5 \\
27.8\end{array}$ & $\begin{array}{l}7.0 \\
5.5 \\
6.4 \\
7.2 \\
4.7\end{array}$ & $\begin{array}{l}15.8 \\
17.7 \\
16.9 \\
14.8 \\
16.8\end{array}$ & $\begin{array}{l}9.6 \\
9.2 \\
8.7 \\
8.2 \\
7.4\end{array}$ & $\begin{array}{l}10.4 \\
12.5 \\
13.6 \\
12.0 \\
12.1\end{array}$ & $\begin{array}{l}14.8 \\
17.9 \\
16.4 \\
18.5 \\
18.3\end{array}$ & $\begin{array}{r}8.2 \\
9.7 \\
11.8 \\
12.7 \\
12.8\end{array}$ \\
\hline $\begin{array}{l}\text { O-6 Bottoms }\left(454^{\circ} \mathrm{C}^{+}\right) \\
\text {O-6 Bottoms }\left(454^{\circ} \mathrm{C}^{+}\right) \\
\text {O-6 Bottoms }\left(454^{\circ} \mathrm{C}^{+}\right) \\
\text {O-6 Bottoms }\left(454^{\circ} \mathrm{C}^{+}\right) \\
\text {O-6 Bottoms }\left(454^{\circ} \mathrm{C}^{+}\right)\end{array}$ & $\begin{array}{r}6 \\
13 \\
17 \\
20 \\
25 \\
\end{array}$ & $\begin{array}{l}40.6 \\
31.1 \\
31.1 \\
33.1 \\
33.9 \\
\end{array}$ & $\begin{array}{l}3.7 \\
8.6 \\
7.5 \\
5.7 \\
6.2 \\
\end{array}$ & $\begin{array}{l}21.2 \\
18.7 \\
18.7 \\
20.0 \\
19.9\end{array}$ & $\begin{array}{r}9.9 \\
10.1 \\
9.9 \\
9.5 \\
9.3 \\
\end{array}$ & $\begin{array}{l}10.5 \\
11.7 \\
11.6 \\
12.0 \\
11.9 \\
\end{array}$ & $\begin{array}{r}9.5 \\
11.8 \\
12.2 \\
12.8 \\
12.2 \\
\end{array}$ & $\begin{array}{l}4.5 \\
7.9 \\
8.9 \\
6.9 \\
6.6 \\
\end{array}$ \\
\hline
\end{tabular}


TABLE 5

PHENOLIC -OH CONCENTRATION IN SAMPLES FROM HTI RUN ALC-1

\begin{tabular}{|c|c|c|c|}
\hline & & \multicolumn{2}{|c|}{ Phenolic -OH } \\
\hline & Period & Conc., meq/g & Peak Pos., cm-1 \\
\hline \multicolumn{4}{|l|}{ Whole Samples } \\
\hline SOH First Stage Oil & 68 & 1.48 & 3304 \\
\hline SOH First Stage Oil & 138 & 2.12 & 3307 \\
\hline SOH First Stage Oil & 17B & 2.04 & 3307 \\
\hline SOH First Stage Oil & $20 B$ & 1.79 & 3307 \\
\hline SOH First Stage Oil & $25 B$ & 1.33 & 3308 \\
\hline SOH Second Stage Oil & 138 & ND & \\
\hline SOH Second Stage Oil & $17 \mathrm{~A}$ & ND & \\
\hline SOH Second Stage Oil & $17 B$ & ND & \\
\hline SOH Second Stage Oil & $20 \mathrm{~A}$ & ND & \\
\hline SOH Second Stage Oil & 208 & ND & \\
\hline SOH Second Stage Oil & $25 B$ & ND & \\
\hline$\overline{\mathrm{VSOH}}$ & $\overline{13 B}$ & 1.17 & $\overline{3311}$ \\
\hline VSOH & $17 \mathrm{~B}$ & 0.91 & 3308 \\
\hline VSOH (IBPX343 C) & $20 \mathrm{~B}$ & 1.48 & 3311 \\
\hline VSOH $(343 \times 399 \mathrm{C})$ & $20 \mathrm{~B}$ & 0.74 & 3306 \\
\hline VSOH, Dewaxed, Hydrotreated (343 C+) & $21 \mathrm{~A}$ & 0.06 & 3314 \\
\hline VSOH, Dewaxed, Hydrotreated, (343 C-) & $21 \mathrm{~A}$ & ND & \\
\hline VSOH (IBPx524 C) & $25 \mathrm{~B}$ & 0.50 & 3304 \\
\hline VSOH, Dewaxed, Hydrotreated & $25 B$ & 0.06 & 3317 \\
\hline VSOH, Dewaxed, Hydrotreated (343 C+) & $25 \mathrm{~B}$ & 0.07 & 3311 \\
\hline VSOH, Dewaxed & $25 \mathrm{~B}$ & 0.54 & 3303 \\
\hline Wax & $25 B$ & 0.23 & 3310 \\
\hline L-814 Start-Up Oil & $6 \bar{A}$ & 0.12 & 3307 \\
\hline$\overline{V S B}$ & 138 & 0.87 & 3297 \\
\hline VSB & $17 \mathrm{~B}$ & 0.92 & 3296 \\
\hline VSB & 208 & 0.81 & 3297 \\
\hline \multicolumn{4}{|l|}{ Distillates } \\
\hline 0.6 Btms (454 C-) & 68 & 1.08 & 3304 \\
\hline $0-6$ Btms (454 C-) & $13 B$ & 1.04 & 3304 \\
\hline O-6 Btms (454 C-) & $17 \mathrm{~B}$ & 0.97 & 3305 \\
\hline $0-6$ Btms (454 C-) & $20 B$ & 0.82 & 3304 \\
\hline $0-6$ Btms (454 C-) & $25 B$ & 0.58 & 3307 \\
\hline \multicolumn{4}{|l|}{ Resid THF Sols } \\
\hline 0.6 8tms $(454 \mathrm{C}+)$ & 68 & 0.94 & 3291 \\
\hline $0-6$ Btms (454 C+) & $13 B$ & 1.00 & 3298 \\
\hline $0-6$ Btms (454 C+) & $17 \mathrm{~B}$ & 0.88 & 3292 \\
\hline $0.6 \mathrm{Btms}(454 \mathrm{C}+)$ & 208 & 0.81 & 3292 \\
\hline $0-6 \mathrm{Btms}(454 \mathrm{C}+)$ & $25 \mathrm{~B}$ & 0.77 & 3294 \\
\hline Feed Slurry $(524 \mathrm{C}+)$ & $\overline{5 A}$ & $\overline{0.79}$ & 3289 \\
\hline Feed Slurry $(524 \mathrm{C}+)$ & $6 A$ & 0.86 & 3287 \\
\hline Feed Slurry $(524 \mathrm{C}+)$ & $5-5-96$ & 0.78 & 3291 \\
\hline Feed Slurry (524 C+) & $13 \mathrm{~B}$ & 0.78 & 3289 \\
\hline Feed Slurry (524 C+) & $17 \mathrm{~B}$ & 0.90 & 3289 \\
\hline Feed Slurry $\left(524 \mathrm{C}^{+}\right)$ & $20 \mathrm{~A}$ & 0.75 & 3289 \\
\hline Feed Slurry (524 C+) & $25 \mathrm{~A}$ & 0.63 & 3288 \\
\hline \multicolumn{4}{|l|}{ THF-Sols From Whole Samples } \\
\hline PFC & $13 A$ & 0.87 & 3298 \\
\hline PFC & 138 & 0.86 & 3299 \\
\hline PFC & $17 \mathrm{~A}$ & 0.82 & 3298 \\
\hline PFC & 258 & 0.67 & 3298 \\
\hline
\end{tabular}

$\mathrm{ND}=$ Not Detected 
TABLE 6

MICROAUTOCLAVE CONVERSIONS

HTI Run ALC-1

\begin{tabular}{|c|c|c|c|}
\hline \multirow[b]{2}{*}{ Sample Type } & \multirow[b]{2}{*}{ Period } & \multicolumn{2}{|c|}{ Conversion, \% (a) } \\
\hline & & Whole Sample & $454^{\circ} \mathrm{C}^{-}$Distillate \\
\hline O-6 Bottoms & 6 & 71.1 & 88.7 \\
\hline O-6 Bottoms & 13 & 76.3 & 88.1 \\
\hline O-6 Bottoms & 17 & 77.1 & 87.6 \\
\hline $0-6$ Bottoms & 20 & 74.3 & 86.7 \\
\hline O-6 Bottoms & 25 & 79.6 & 88.4 \\
\hline VSOH, Dewaxed Hydrotreated, $343^{\circ} \mathrm{C}^{+}$ & $21 \mathrm{~A}$ & 92.7 & - \\
\hline VSOH, Dewaxed & 25 & 89.7 & - \\
\hline VSOH, IBP $\times 524^{\circ} \mathrm{C}$ & 25 & 89.5 & - \\
\hline VSOH, Dewaxed Hydrotreated, $343^{\circ} \mathrm{C}^{+}$ & 25 & 94.4 & - \\
\hline VSOH, Hydrotreated, Dewaxed & 25 & 92.6 & - \\
\hline VSOH, Dewaxed (acetone free) Can $\# 2$ & (b) & 86.8 & 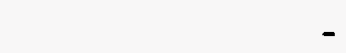 \\
\hline L-814 Start-Up Oil & 6 & 86.8 & \\
\hline Feed Slurry & 13 & - & $81.4(\mathrm{c})$ \\
\hline Feed Slurry & 20 & & $84.4(c)$ \\
\hline
\end{tabular}

(a) Microautoclave coal conversion, wt \% MAF, in modified equilibrium test, Old Ben Mine coal

(b) Pre-run make-up

(c) Distillate was $343 \times 524{ }^{\circ} \mathrm{C}$

TABLE 7

COMPOSITION OF SOH WATER PRODUCTS FROM HTI RUN ALC-1

\begin{tabular}{|c|c|c|c|c|c|}
\hline \multicolumn{2}{|c|}{ SOH Water Sample Source } & \multirow[b]{2}{*}{$\mathrm{pH}$} & \multicolumn{3}{|c|}{ Elemental Composition, wt \% of Sample } \\
\hline Period & Stage & & $S(a)$ & $N(b)$ & $c(b)$ \\
\hline $6 \mathrm{~B}$ & First & 9.22 & 0.79 & 1.52 & 1.72 \\
\hline $\begin{array}{l}13 B \\
13 B\end{array}$ & $\begin{array}{l}\text { First } \\
\text { Second }\end{array}$ & $\begin{array}{l}9.07 \\
9.74\end{array}$ & $\begin{array}{l}1.24 \\
2.69\end{array}$ & $\begin{array}{l}1.77 \\
2.14\end{array}$ & $\begin{array}{l}1.84 \\
0.54\end{array}$ \\
\hline $\begin{array}{l}17 \mathrm{~B} \\
17 \mathrm{~B}\end{array}$ & $\begin{array}{l}\text { First } \\
\text { Second }\end{array}$ & $\begin{array}{l}9.19 \\
9.21\end{array}$ & $\begin{array}{l}1.07 \\
2.50\end{array}$ & $\begin{array}{l}1.43 \\
2.00\end{array}$ & $\begin{array}{l}1.69 \\
0.27\end{array}$ \\
\hline $\begin{array}{l}20 \mathrm{~B} \\
20 \mathrm{~B}\end{array}$ & $\begin{array}{c}\text { First } \\
\text { Second }\end{array}$ & $\begin{array}{l}9.02 \\
9.29\end{array}$ & $\begin{array}{l}1.05 \\
2.52\end{array}$ & $\begin{array}{l}1.46 \\
1.85\end{array}$ & $\begin{array}{l}1.70 \\
0.12\end{array}$ \\
\hline $\begin{array}{l}25 B \\
25 B\end{array}$ & $\begin{array}{l}\text { First } \\
\text { Second }\end{array}$ & $\begin{array}{l}8.94 \\
9.09\end{array}$ & $\begin{array}{l}0.66 \\
2.22\end{array}$ & $\begin{array}{l}1.25 \\
1.63\end{array}$ & $\begin{array}{l}1.71 \\
0.15\end{array}$ \\
\hline
\end{tabular}

(a) Total S analysis by LECO SC-32 Sulfur Analyzer

(b) $\mathrm{C}$ and $\mathrm{N}$ analysis by LECO $\mathrm{CHN}-1000$ Analyzer 
TABLE 8

\section{CONCENTRATION OF COMPONENTS OF THE FEED SLURRY SAMPLES FROM HTI RUN ALC-1}

\begin{tabular}{|l|c|c|c|c|c|c|c||}
\hline Date, 1996 & $\mathbf{4 / 2 3}$ & $\mathbf{4 / 2 4}$ & $\mathbf{5 / 1}$ & $\mathbf{5 / 5}$ & $\mathbf{5 / 8}$ & $\mathbf{5 / 1 3}$ & $\mathbf{5 / 5}(\mathrm{a})$ \\
\hline Period & $\mathbf{5 A}$ & $\mathbf{6 A}$ & $\mathbf{1 3 A}$ & $\mathbf{1 7 A}$ & $\mathbf{2 0 A}$ & $\mathbf{2 5 A}$ & $\mathbf{1 3 A}(\mathrm{a})$ \\
\hline Component, wt \% & & & & & & & \\
\hline$-343^{\circ} \mathrm{C}$ & $4.3(\mathrm{~b})$ & $5.2(\mathrm{~b})$ & trace & $0.9(\mathrm{~b})$ & 0.0 & $4.2(\mathrm{~b})$ & trace \\
$343^{\circ} \mathrm{C} \times 524^{\circ} \mathrm{C}$ & 6.7 & $28.7(\mathrm{c})$ & 15.7 & 20.7 & 19.1 & 28.5 & 16.3 \\
THF-Soluble $524{ }^{\circ} \mathrm{C}^{+}$ & 52.5 & 16.4 & 31.9 & 24.9 & 27.8 & 16.0 & 39.7 \\
IOM & 28.8 & 39.0 & 41.1 & 45.3 & 43.6 & 41.6 & 36.1 \\
Ash & 6.8 & 8.9 & 6.9 & 7.8 & 7.8 & 8.2 & 5.0 \\
Total & 99.1 & 98.2 & 95.6 & 99.6 & 98.3 & 98.5 & 97.1 \\
\hline
\end{tabular}
(a) Date/period in question
(b) May have contained an unknown amount of water
(c) End point was ca. $529^{\circ} \mathrm{C}\left(985^{\circ} \mathrm{F}\right)$, instead of $524^{\circ} \mathrm{C}\left(975^{\circ} \mathrm{F}\right)$

Actual distillation conditions were 5 torr $/ 3488^{\circ} \mathrm{F}$ pot/378 ${ }^{\circ} \mathrm{F}$ head $\left(176{ }^{\circ} \mathrm{C}\right.$ pot/192 ${ }^{\circ} \mathrm{C}$ head) and 5 torr $/ 608^{\circ} \mathrm{F}$ pot'638 ${ }^{\circ} \mathrm{F}$ head $\left(320^{\circ} \mathrm{C}\right.$ pot $337^{\circ} \mathrm{C}$ head) to provide effective cut points of $343^{\circ} \mathrm{C}\left(650^{\circ} \mathrm{F}\right)$ and $524^{\circ} \mathrm{C}\left(975^{\circ} \mathrm{F}\right)$.

TABLE 9

\section{ASH ELEMENTAL COMPOSITION OF PRESSURE-FILTER CAKES FROM HTI RUN ALC-1}

\begin{tabular}{|c|c|c|c|c|c|}
\hline & $\begin{array}{l}\text { Period 6 } \\
\text { (Toluene- } \\
\text { extracted) }\end{array}$ & $\begin{array}{c}\text { Period } 13 \\
\text { (Unextracted) }\end{array}$ & $\begin{array}{l}\text { Period } 17 \\
\text { (Unextracted) }\end{array}$ & $\begin{array}{l}\text { Period 20 } \\
\text { (Unextracted) }\end{array}$ & $\begin{array}{c}\text { Period } 25 \\
\text { (Unextracted) }\end{array}$ \\
\hline $\begin{array}{l}\text { Moisture, wt } \% \text { As-Determined } \\
\text { Ash, wt } \% \mathrm{MF} \text {, Including } \mathrm{SO}_{3} \\
\text { Ash, wt } \% \mathrm{MF}, \mathrm{SO}_{3}-\text { Free }\end{array}$ & $\begin{array}{r}0.30 \\
70.45 \\
51.58\end{array}$ & $\begin{array}{r}0.23 \\
47.49 \\
42.60\end{array}$ & $\begin{array}{r}0.31 \\
45.24 \\
40.58\end{array}$ & $\begin{array}{r}0.43 \\
45.61 \\
41.19\end{array}$ & $\begin{array}{r}0.41 \\
45.20 \\
31.64\end{array}$ \\
\hline $\begin{array}{l}\text { Major Ash Elements, Oxide } \\
\mathrm{Wt} \% \text { of Ash } \\
\mathrm{MoO}_{2} \\
\mathrm{Na}_{2} \mathrm{O} \\
\mathrm{K}_{2} \mathrm{O} \\
\mathrm{CaO} \\
\mathrm{MgO} \\
\mathrm{Fe}_{2} \mathrm{O}_{3} \\
\mathrm{TiO}_{2} \\
\mathrm{P}_{2} \mathrm{O}_{5} \\
\mathrm{SiO}_{2} \\
\mathrm{Al}_{2} \mathrm{O}_{3} \\
\mathrm{SO}_{3} \\
\mathrm{Total}\end{array}$ & $\begin{array}{r}0.21 \\
1.17 \\
0.29 \\
14.76 \\
3.29 \\
22.27 \\
0.77 \\
0.81 \\
19.03 \\
10.34 \\
26.78 \\
99.72\end{array}$ & $\begin{array}{r}0.31 \\
0.37 \\
0.33 \\
6.47 \\
1.14 \\
36.00 \\
1.42 \\
1.18 \\
27.76 \\
15.11 \\
10.29 \\
100.38\end{array}$ & $\begin{array}{r}0.49 \\
0.37 \\
0.41 \\
6.86 \\
1.14 \\
30.45 \\
1.58 \\
1.33 \\
30.60 \\
16.76 \\
10.31 \\
100.30\end{array}$ & $\begin{array}{r}0.56 \\
0.32 \\
0.37 \\
6.52 \\
1.02 \\
26.93 \\
1.61 \\
1.33 \\
32.05 \\
17.97 \\
9.68 \\
98.36\end{array}$ & $\begin{array}{r}0.21 \\
1.17 \\
0.27 \\
17.45 \\
3.58 \\
17.94 \\
1.02 \\
1.00 \\
15.24 \\
11.55 \\
29.99 \\
99.42\end{array}$ \\
\hline $\begin{array}{l}\text { Mo wt } \% \text { of SO3-free ash } \\
\text { Fe wt } \% \text { of SO3-free ash }\end{array}$ & $\begin{array}{r}0.22 \\
21.27\end{array}$ & $\begin{array}{r}0.26 \\
28.07\end{array}$ & $\begin{array}{r}0.41 \\
23.75\end{array}$ & $\begin{array}{r}0.47 \\
20.85\end{array}$ & $\begin{array}{r}0.23 \\
17.92\end{array}$ \\
\hline $\begin{array}{l}\text { Mo wt } \% \text { of SO3-containing ash } \\
\text { Fe wt } \% \text { of SO3-containing ash }\end{array}$ & $\begin{array}{r}0.16 \\
15.58\end{array}$ & $\begin{array}{r}0.23 \\
25.18\end{array}$ & $\begin{array}{r}0.37 \\
21.30\end{array}$ & $\begin{array}{r}0.42 \\
18.84\end{array}$ & $\begin{array}{r}0.16 \\
12.55\end{array}$ \\
\hline \multicolumn{6}{|c|}{ HTI Analyses, wt $\%$ of ASTM Ash (a) } \\
\hline $\begin{array}{l}\text { Mo - Second Stage Sample } \\
\text { Fe - Second Stage Sample }\end{array}$ & $\begin{array}{l}0.195 \\
9.226\end{array}$ & $\begin{array}{c}0.232 \\
21.098\end{array}$ & $\begin{array}{l}0.400 \\
18.766\end{array}$ & $\begin{array}{c}0.403 \\
17.372\end{array}$ & $\begin{array}{c}0.190 \\
13.206\end{array}$ \\
\hline $\begin{array}{l}\text { Mo - First Stage Sample } \\
\text { Fe - First Stage Sample }\end{array}$ & $\begin{array}{c}0.198 \\
13.316\end{array}$ & $\begin{array}{r}0.188 \\
17.468\end{array}$ & $\begin{array}{r}0.218 \\
12.721\end{array}$ & $\begin{array}{r}0.232 \\
12.202\end{array}$ & $\begin{array}{r}0.162 \\
10.708\end{array}$ \\
\hline
\end{tabular}

(a) Source: Table 16 (page 40) of the HTI draft Run ALC-1 report of June 1996 (early draft of Reference 1). 
TABLE 10

ASH ELEMENTAL COMPOSITION OF FEED SLURRY $524^{\circ} \mathrm{C}+$ RESIDS FROM HTI RUN ALC-1

\begin{tabular}{|c|c|c|c|c|c|c|}
\hline & Period 5 & Period 6 & Period 13 & Period 17 & Period 20 & $\begin{array}{l}\text { Period } 25 \\
\text { (a) }\end{array}$ \\
\hline $\begin{array}{l}\text { Moisture, wt \% As-Determined } \\
\text { Ash, wt } \% \mathrm{MF} \text {, Including } \mathrm{SO}_{3} \\
\text { Ash, wt } \% \mathrm{MF}, \mathrm{SO}_{3} \text {-Free }\end{array}$ & $\begin{array}{l}0.26 \\
8.12 \\
6.06\end{array}$ & $\begin{array}{r}2.14 \\
15.26 \\
11.42\end{array}$ & $\begin{array}{r}1.48 \\
10.29 \\
9.27\end{array}$ & $\begin{array}{r}1.25 \\
10.18 \\
9.26\end{array}$ & $\begin{array}{r}0.96 \\
10.02 \\
9.16\end{array}$ & $\begin{array}{r}2.16 \\
13.20 \\
9.90\end{array}$ \\
\hline $\begin{array}{l}\text { Maior Ash Elements, Oxide } \\
\mathrm{wt} \% \text { of Ash } \\
\mathrm{MoO}_{2} \\
\mathrm{Na}_{2} \mathrm{O} \\
\mathrm{K}_{2} \mathrm{O} \\
\mathrm{CaO} \\
\mathrm{MgO} \\
\mathrm{Fe}_{2} \mathrm{O}_{3} \\
\mathrm{TiO}_{2} \\
\mathrm{P}_{2} \mathrm{O}_{5} \\
\mathrm{SiO}_{2} \\
\mathrm{Al}_{2} \mathrm{O}_{3} \\
\mathrm{SO}_{3} \\
\text { Total }\end{array}$ & $\begin{array}{r}0.20 \\
1.15 \\
0.29 \\
14.53 \\
3.16 \\
23.13 \\
0.79 \\
0.80 \\
19.76 \\
10.73 \\
25.41 \\
99.95\end{array}$ & $\begin{array}{r}0.20 \\
1.17 \\
0.29 \\
14.41 \\
3.18 \\
23.64 \\
0.78 \\
0.82 \\
19.08 \\
11.04 \\
25.16 \\
99.77\end{array}$ & $\begin{array}{r}0.43 \\
0.43 \\
0.33 \\
6.88 \\
1.25 \\
33.63 \\
1.41 \\
1.16 \\
28.28 \\
15.94 \\
9.90 \\
99.64\end{array}$ & $\begin{array}{r}0.48 \\
0.38 \\
0.39 \\
6.57 \\
1.09 \\
30.29 \\
1.59 \\
1.31 \\
30.92 \\
17.38 \\
9.03 \\
99.43\end{array}$ & $\begin{array}{r}0.56 \\
0.31 \\
0.36 \\
6.14 \\
0.98 \\
29.37 \\
1.57 \\
1.27 \\
31.64 \\
17.74 \\
8.60 \\
98.54\end{array}$ & $\begin{array}{r}0.20 \\
1.18 \\
0.28 \\
16.44 \\
3.43 \\
19.17 \\
1.02 \\
0.99 \\
19.63 \\
12.37 \\
25.01 \\
99.72\end{array}$ \\
\hline $\begin{array}{l}\text { Mo wt } \% \text { of } \mathrm{SO}_{3} \text {-free ash } \\
\mathrm{Fe} w \mathrm{t} \% \text { of } \mathrm{SO}_{3} \text {-free ash }\end{array}$ & $\begin{array}{r}0.20 \\
21.69\end{array}$ & $\begin{array}{r}0.20 \\
22.09\end{array}$ & $\begin{array}{r}0.36 \\
26.11\end{array}$ & $\begin{array}{r}0.40 \\
23.29\end{array}$ & $\begin{array}{r}0.46 \\
22.47\end{array}$ & $\begin{array}{r}0.20 \\
17.88\end{array}$ \\
\hline $\begin{array}{l}\text { Mo wt } \% \text { of } \mathrm{SO}_{3} \text {-containing ash } \\
\text { Fe wt } \% \text { of } \mathrm{SO}_{3} \text {-containing ash }\end{array}$ & $\begin{array}{r}0.15 \\
16.18\end{array}$ & $\begin{array}{r}0.15 \\
16.53\end{array}$ & $\begin{array}{r}0.32 \\
23.52\end{array}$ & $\begin{array}{r}0.36 \\
21.19\end{array}$ & $\begin{array}{r}0.42 \\
20.54\end{array}$ & $\begin{array}{r}0.14 \\
13.41\end{array}$ \\
\hline
\end{tabular}

(a) Single determination, others are averages of duplicate determinations. 
TABLE 11

\section{Mo AND Fe BALANCE AND APPARENT ADDITION RATES FOR HTI RUN ALC-1}

\begin{tabular}{|c|c|c|c|c|c|c|c|c|}
\hline \multicolumn{9}{|c|}{ TOTAL Mo \& Fe in Feed Slurry, (Based on coal ash and recycle $10 \mathrm{M}+$ ash rates): } \\
\hline Period & $\begin{array}{l}\text { MF Coal } \\
\text { In, g }\end{array}$ & $\begin{array}{l}\mathrm{Fe}_{2} \mathrm{O}_{3} \\
\mathrm{~g}\end{array}$ & $\begin{array}{c}\text { Coal } \\
\text { Ash In, g }\end{array}$ & $\begin{array}{l}\text { Coal + Cat } \\
\text { Ash In, g }\end{array}$ & $\begin{array}{l}\text { Recycle } \\
\text { Ash In, } g\end{array}$ & $\begin{array}{c}\text { Total Ash } \\
\text { In, } g\end{array}$ & $\begin{array}{l}\text { Total Mo } \\
\text { In, } g\end{array}$ & $\begin{array}{l}\text { Total Fe } \\
\text { In, } g\end{array}$ \\
\hline 6 & 32,405 & $\overline{454}$ & 1,782 & 2,236 & 4,439 & 6,675 & 13.35 & 1,475 \\
\hline 13 & 26,786 & 375 & 884 & 1,259 & 3,536 & 4,795 & 17.26 & $\overline{1,252}$ \\
\hline 17 & 26,365 & 258 & 870 & 1,128 & 3,691 & 4,820 & 19.28 & 1,122 \\
\hline 20 & 20,078 & 197 & 663 & 859 & 2,570 & 3,429 & 15.77 & $\overline{771}$ \\
\hline 25 & 22,362 & 219 & 1,230 & 1,449 & 2,303 & 3,752 & 7.50 & $\overline{671}$ \\
\hline \multicolumn{9}{|c|}{ Mo \& Fe in TOTAL PFC OUT: } \\
\hline Period & $\begin{array}{l}\text { Tot. PFC } \\
\text { Out, } \mathrm{g}\end{array}$ & $\begin{array}{l}\text { PFC Ash } \\
\text { Out, } g\end{array}$ & $\begin{array}{c}\text { PFC Mo Out } \\
\mathbf{g}\end{array}$ & $\begin{array}{l}\text { PFC Fe } \\
\text { Out, } g\end{array}$ & $\begin{array}{l}\text { Mo Out/ln, } \\
\text { Ratio }\end{array}$ & $\begin{array}{c}\text { Fe Out/ln, } \\
\text { Ratio }\end{array}$ & & \\
\hline 6 & 13,215 & 6,816 & 15.00 & 1,450 & 1.12 & 0.98 & & \\
\hline$\overline{13}$ & 12,537 & 5,341 & 13.89 & 1,499 & 0.80 & 1.20 & & \\
\hline 17 & 11,775 & 4,778 & 19.59 & 1,135 & 1.02 & 1.01 & & \\
\hline 20 & 8,919 & 3,674 & 17.27 & 766 & 1.09 & 0.99 & & \\
\hline 25 & 12,307 & 3,894 & 8.96 & 698 & 1.19 & 1.04 & & \\
\hline \multicolumn{9}{|c|}{ Mo \& Fe from RECYCLE PFC: } \\
\hline Period & $\begin{array}{c}\text { PFC } \\
\text { Recycle, } g\end{array}$ & $\begin{array}{l}\text { PFC Ash } \\
\text { Recycle, } g\end{array}$ & $\begin{array}{l}\text { PFC Mo } \\
\text { Recycle, g }\end{array}$ & $\begin{array}{c}\text { PFC Fe } \\
\text { Recycle, } \mathrm{g}\end{array}$ & $\begin{array}{l}\text { Mo Rec/An, } \\
\text { Ratio }\end{array}$ & $\begin{array}{c}\mathrm{Fe} \text { Rec/in, } \\
\text { Ratio }\end{array}$ & & \\
\hline 6 & 8,638 & 4,455 & 9.80 & 948 & 0.73 & 0.64 & & \\
\hline 13 & 9,084 & 3,870 & 10.06 & 1,086 & 0.58 & 0.87 & & \\
\hline 17 & 9,030 & 3,664 & 15.02 & 870 & 0.78 & 0.78 & & \\
\hline 20 & 6,618 & 2,726 & 12.81 & 568 & 0.81 & 0.74 & & \\
\hline 25 & 7,420 & 2,348 & 5.40 & 421 & 0.72 & 0.63 & & \\
\hline \multicolumn{9}{|c|}{ Mo \& Fe NET in Fresh Feed: } \\
\hline Period & $\begin{array}{c}\text { Total Mo } \\
\text { In, } g\end{array}$ & $\begin{array}{l}\text { Total } \mathrm{Fe} \\
\text { In, } \mathrm{g}\end{array}$ & $\begin{array}{l}\text { PFC Mo } \\
\text { Recycle, g }\end{array}$ & $\begin{array}{l}\text { PFC Fe } \\
\text { Recycle, g }\end{array}$ & $\begin{array}{l}\text { Calc. } \\
\text { Fresh Mo } \\
\text { In, g }\end{array}$ & $\begin{array}{l}\text { Calc. } \\
\text { Fresh Fe } \\
\text { In, } g\end{array}$ & $\begin{array}{l}\text { Calc. Fresh } \\
\text { Mo Rate, } \\
\text { ppm }\end{array}$ & $\begin{array}{l}\text { Target Mo } \\
\text { Rate, ppm }\end{array}$ \\
\hline 6 & 13.35 & 1,475 & 9.80 & 948 & 3.55 & 527 & 110 & 100 \\
\hline 13 & 17.26 & 1,252 & 10.06 & 1,086 & 7.20 & 166 & 269 & 100 \\
\hline 17 & 19.28 & 1,122 & 15.02 & 870 & 4.25 & 252 & 161 & 70 \\
\hline 20 & 15.77 & $\overline{771}$ & 12.81 & 568 & 2.96 & 202 & 148 & 70 \\
\hline 25 & 7.50 & 671 & 5.40 & 421 & 2.11 & 250 & 94 & 70 \\
\hline Period & $\begin{array}{c}\text { Calc Fe } \\
\text { Total Rate, } \\
\text { ppm }\end{array}$ & $\begin{array}{c}\text { Fe from } \\
\text { Coal, ppm }\end{array}$ & $\begin{array}{l}\text { Calc. Fresh } \\
\text { Added Fe } \\
\text { Rate, ppm }\end{array}$ & $\begin{array}{c}\text { Target } \\
\text { Added Fe } \\
\text { Rate, ppm }\end{array}$ & & & & \\
\hline 6 & 16,261 & 2,618 & 13,642 & 10,000 & & & & \\
\hline 13 & 6,184 & 2,256 & 3,928 & 10,000 & & & & \\
\hline 17 & 9,565 & 2,256 & 7,309 & 7,000 & & & & \\
\hline 20 & 10,071 & 2,256 & 7,815 & 7,000 & & & & \\
\hline 25 & 11,189 & 2,618 & 8,571 & 7,000 & & & & \\
\hline
\end{tabular}

All ppm values are on a ppm (mg/kg) MF coal basis. 
TABLE 12

WAX CONTENT IN VSOH

HTI RUN ALC-1

\begin{tabular}{||c|c|c|c|c||}
\hline \multirow{2}{*}{ Stream } & \multirow{3}{*}{} & \multicolumn{3}{|c||}{ Wax Yield at Temperature (a), \% } \\
\cline { 3 - 5 } & Period & $\mathbf{- 5}{ }^{\circ} \mathbf{C}$ & $\mathbf{- 2 0}{ }^{\circ} \mathbf{C}$ & $\mathbf{- 3 5}{ }^{\circ} \mathbf{C}$ \\
\hline O-6 Bottoms Distillate & 20 & 5.2 & 5.9 & 6.1 \\
VSOH & 25 & 1.2 & 1.5 & 2.6 \\
VSOH, dewaxed & 25 & 0.1 & 0.1 & 0.2 \\
\hline
\end{tabular}

(a) Yield of wax obtained in laboratory dewaxing test with acetone at the specified temperature. 


\section{TABLE 13}

\section{SUMMARY OF ${ }^{1} \mathrm{H}-N M R$ ANALYSES AND MICROAUTOCLAVE TESTS RELEVANT TO RUN ALC-1 DEWAXING OPERATIONS}

\begin{tabular}{|c|c|c|c|c|c|c|c|}
\hline \multirow[b]{2}{*}{ Period } & \multirow[b]{2}{*}{ Stream } & \multicolumn{3}{|c|}{ 'H-NMR Proton Types, \% } & \multicolumn{2}{|c|}{$\begin{array}{l}\text { Microautoclave Coal } \\
\text { Conversion, \% MAF }\end{array}$} & \multirow{2}{*}{$\begin{array}{c}\text { Phenolic -OH } \\
\text { Concentration, } \\
\text { meq/g }\end{array}$} \\
\hline & & Aromatic & Cyclic & Paraffinic & $\begin{array}{l}\text { Test A } \\
\text { (a) }\end{array}$ & $\begin{array}{l}\text { Test B } \\
\text { (b) }\end{array}$ & \\
\hline $\begin{array}{l}13,17,20 \\
25 \\
25 \\
25 \\
25\end{array}$ & $\begin{array}{l}\text { VSOH/O-6 Bottoms Distillate } \\
\text { VSOH/O-6 Bottoms Distillate } \\
\text { VSOH, dewaxed } \\
\text { VSOH, dewaxed and hydrotreated } \\
\text { VSOH, dewaxed and hydrotreated, } \\
343^{\circ} \mathrm{C}^{+}\left(650^{\circ} \mathrm{F}\right)\end{array}$ & $\begin{array}{c}24.5 \pm 1.4 \\
23.7 \pm 0.3 \\
27.6 \\
14.3 \\
17.1\end{array}$ & $\begin{array}{l}28.7 \pm 0.6 \\
31.6 \pm 1.7 \\
31.6 \\
37.2 \\
38.1\end{array}$ & $\begin{array}{l}35.4 \pm 1.2 \\
33.4 \pm 1.4 \\
29.5 \\
38.6 \\
34.3\end{array}$ & $\begin{array}{l}87.4 \pm 0.7 \\
89.0 \pm 0.8 \\
89.7 \\
92.6 \\
94.4\end{array}$ & $\begin{array}{c}78.3(c) \\
80.9 / 79.4 \\
82.3 \\
89.9 \\
90.5\end{array}$ & $\begin{array}{l}0.98 \pm 0.13 \\
0.54 \pm 0.06 \\
0.54 \\
0.06 \\
0.07\end{array}$ \\
\hline
\end{tabular}

(a) Modified equilibrium tests with Old Ben Mine coal

(b) Black Thunder Mine coal $(4.55 \mathrm{~g})$, solvent $(5.45 \mathrm{~g}), 30 \mathrm{~min}, 440^{\circ} \mathrm{C}\left(824^{\circ} \mathrm{F}\right), 10.3 \mathrm{MPa}(1500 \mathrm{psig}) \mathrm{H}_{2}$ (cold)

(c) Data shown from period $200-6$ bottoms sample

TABLE 14

ANALYSIS AND DE-OILING YIELDS OF WAX FROM RUN ALC-1, PERIOD 25

\begin{tabular}{|l|c|}
\hline & wt \% (a) \\
\hline Elemental Analysis of Original Wax & \\
\hline$C$ & $73.82(\mathrm{~b})$ \\
$\mathrm{H}$ & 13.70 \\
$\mathrm{~N}$ & 0.20 \\
$\mathrm{~S}$ & 0.06 \\
\hline
\end{tabular}

(a) Average of four determinations. Repeatability was ca. $\pm 5 \%$ relative.

(b) Suspect low, cf. text.

\begin{tabular}{||c|c|}
\hline \multicolumn{2}{|c|}{ Yields from De-Oiling Original Wax } \\
\hline \multicolumn{1}{|c|}{ Fraction } & wt \% \\
\hline De-Oiled Wax & 74.7 \\
Oil & 19.4 \\
Insolubles & 1.7 \\
Losses & 4.2 \\
\hline
\end{tabular}

\begin{tabular}{||c|c|c|c|c|c|c|c||}
\hline \multicolumn{8}{|c||}{ Proton Distributions of De-Oiling Fractions } \\
\hline & \multicolumn{7}{|c|}{ Proton Distribution, \% } \\
\cline { 2 - 8 } & Cond & $\begin{array}{c}\text { Uncond } \\
\text { Arom }\end{array}$ & $\begin{array}{c}\text { Cyclic } \\
\text { Alpha }\end{array}$ & $\begin{array}{c}\text { Alkyl } \\
\text { Alpha }\end{array}$ & $\begin{array}{c}\text { Cyclic } \\
\text { Beta }\end{array}$ & $\begin{array}{c}\text { Alkyl } \\
\text { Beta }\end{array}$ & Gamma \\
\hline Original Wax & 2.5 & 1.4 & 4.6 & 3.2 & 8.1 & 68.7 & 11.5 \\
De-Oiled Wax & 0.5 & 0.2 & 0.6 & 0.7 & 3.3 & 80.4 & 14.4 \\
Oil & 8.9 & 3.3 & 8.5 & 5.5 & 10.6 & 49.9 & 13.3 \\
\hline
\end{tabular}


TABLE 15

\section{CONSOL ANALYSES OF PB-03 CRUDE OIL ASSAY FRACTIONS}

\begin{tabular}{|c|c|c|c|c|c|c|c|c|c|}
\hline & \multicolumn{7}{|c|}{ Proton Distribution, $\%$} & \multicolumn{2}{|c|}{ Phenolic -OH } \\
\hline & $\begin{array}{l}\text { Cond } \\
\text { Arom }\end{array}$ & $\begin{array}{l}\text { Uncond } \\
\text { Aom }\end{array}$ & $\begin{array}{l}\text { Cyclic } \\
\text { Alpha }\end{array}$ & $\begin{array}{l}\text { Alkyl } \\
\text { Alpha }\end{array}$ & $\begin{array}{c}\text { Cyclic } \\
\text { Beta }\end{array}$ & $\begin{array}{l}\text { Alkyl } \\
\text { Beta }\end{array}$ & Gamma & $\begin{array}{l}\text { Conc., } \\
\text { meq/g }\end{array}$ & $\begin{array}{l}\text { Peak, } \\
\mathrm{cm}^{-1}\end{array}$ \\
\hline $\begin{array}{l}\text { PB-03-6.7,8 } \\
70 \times 180^{\circ} \mathrm{F} \\
180 \times 350^{\circ} \mathrm{F} \\
400 \times 550^{\circ} \mathrm{F} \\
550 \times 650^{\circ} \mathrm{F} \\
650^{\circ} \mathrm{F}^{+}\end{array}$ & $\begin{array}{l}1.1 \\
1.0 \\
1.1 \\
1.8 \\
2.6\end{array}$ & $\begin{array}{l}0.4 \\
2.1 \\
5.9 \\
4.4 \\
2.6\end{array}$ & $\begin{array}{l}2.8 \\
3.7 \\
8.6 \\
8.9 \\
7.3\end{array}$ & $\begin{array}{r}2.7 \\
4.4 \\
8.2 \\
16.1 \\
5.4\end{array}$ & $\begin{array}{l}30.2 \\
27.3 \\
22.2 \\
20.2 \\
16.8\end{array}$ & $\begin{array}{l}37.6 \\
32.0 \\
31.1 \\
29.6 \\
45.5\end{array}$ & $\begin{array}{l}25.2 \\
29.4 \\
22.8 \\
19.0 \\
19.8\end{array}$ & $\begin{array}{l}B D \\
B D \\
B D \\
B D \\
B D\end{array}$ & - \\
\hline $\begin{array}{l}\text { PB-03-9,10,11 } \\
\text { Crude, as received } \\
70 \times 180^{\circ} \mathrm{F} \\
180 \times 350^{\circ} \mathrm{F} \\
400 \times 550^{\circ} \mathrm{F} \\
550 \times 650^{\circ} \mathrm{F} \\
650^{\circ} \mathrm{F}^{+}\end{array}$ & $\begin{array}{r}5.1 \\
1.0 \\
1.9 \\
4.7 \\
9.4 \\
14.3\end{array}$ & $\begin{array}{r}10.4 \\
0.3 \\
6.7 \\
14.0 \\
8.5 \\
8.3 \\
\end{array}$ & $\begin{array}{r}9.0 \\
1.5 \\
4.0 \\
12.2 \\
14.0 \\
13.8\end{array}$ & $\begin{array}{r}11.1 \\
3.5 \\
5.9 \\
16.0 \\
11.6 \\
10.9 \\
\end{array}$ & $\begin{array}{l}16.9 \\
28.1 \\
22.0 \\
13.9 \\
15.7 \\
12.7 \\
\end{array}$ & $\begin{array}{l}28.3 \\
37.8 \\
31.2 \\
23.9 \\
26.3 \\
26.9 \\
\end{array}$ & $\begin{array}{l}19.1 \\
27.8 \\
28.2 \\
15.2 \\
14.6 \\
13.2 \\
\end{array}$ & $\begin{array}{r}1.69 \\
B D \\
1.15 \\
2.44 \\
0.92 \\
0.75 \\
\end{array}$ & $\begin{array}{r}3309 \\
- \\
3300 \\
3314 \\
3314 \\
3304 \\
\end{array}$ \\
\hline
\end{tabular}

\section{$B D=$ Below Detection Limit}

Temperature Equivalents:
$70^{\circ} \mathrm{F}=21^{\circ} \mathrm{C}$
$180^{\circ} \mathrm{F}=82{ }^{\circ} \mathrm{C}$
$350^{\circ} \mathrm{F}=177^{\circ} \mathrm{C}$
$400^{\circ} \mathrm{F}=204^{\circ} \mathrm{C}$
$550^{\circ} \mathrm{F}=288^{\circ} \mathrm{C}$
$650^{\circ} \mathrm{F}=343^{\circ} \mathrm{C}$ 
TABLE 16

RESID REACTIVITY TESTS

\begin{tabular}{|c|c|c|c|c|c|c|}
\hline Run No. & $\begin{array}{l}\text { RR-8 } \\
8 / 19 / 96\end{array}$ & $\begin{array}{l}\text { RR-9 } \\
8 / 21 / 96\end{array}$ & $\begin{array}{c}R R-10 \\
8 / 22 / 96\end{array}$ & $\begin{array}{l}\text { RR-11 } \\
8 / 23 / 96\end{array}$ & $\begin{array}{l}R R-12 \\
8 / 26 / 96\end{array}$ & $\begin{array}{l}\text { RR-13 } \\
8 / 27 / 96\end{array}$ \\
\hline $\begin{array}{l}\text { Feed } \\
\text { Resid Source } \\
\text { Resid, } g \\
\text { Mo Naphthenate, } g \\
\text { DMDS, g } \\
\text { Tetralin, } g \\
\mathrm{H}_{2}, \mathrm{~g}\end{array}$ & $\begin{array}{r}W 259 \text { V1067 } \\
4.00 \\
- \\
- \\
8.00 \\
0.33\end{array}$ & $\begin{array}{r}\text { W259 V1067 } \\
4.00 \\
2.00 \\
0.50 \\
8.00 \\
0.28\end{array}$ & $\begin{array}{r}\text { W261 R1235 } \\
4.00 \\
2.00 \\
0.50 \\
8.00 \\
0.37\end{array}$ & $\begin{array}{r}\text { W261 R1235 } \\
4.00 \\
- \\
8.00 \\
0.24\end{array}$ & $\begin{array}{r}W 259 \text { V131B } \\
4.00 \\
2.00 \\
0.50 \\
8.00 \\
0.32\end{array}$ & $\begin{array}{r}W 260 V 131 B \\
4.00 \\
2.00 \\
0.50 \\
8.00 \\
0.28\end{array}$ \\
\hline $\begin{array}{l}\text { Product } \\
\text { Gas, } \mathrm{g} \\
\text { Distillate } \\
\text { Cold Trap 1,g } \\
\text { Cold Trap 2, } \\
\text { Receiver 1,g } \\
\text { Receiver 2, g } \\
\text { Dist. Overhead, g } \\
\text { Dist. Column, g } \\
\text { Reactor Head, g } \\
\text { Reactor Bottom, g }\end{array}$ & $\begin{array}{r}0.06 \\
1.22 \\
6.28 \\
- \\
0.01 \\
0.06 \\
0.29 \\
3.78\end{array}$ & $\begin{array}{l}0.39 \\
0.33 \\
1.36 \\
0.14 \\
5.65 \\
0.10 \\
0.61 \\
0.87 \\
3.71\end{array}$ & $\begin{array}{r}0.43 \\
0.95 \\
1.43 \\
7.42 \\
- \\
0.05 \\
0.45 \\
0.30 \\
3.32\end{array}$ & $\begin{array}{r}0.13 \\
0.87 \\
0.30 \\
6.71 \\
- \\
0.01 \\
0.05 \\
0.15 \\
3.66\end{array}$ & $\begin{array}{l}0.51 \\
\\
0.52 \\
0.20 \\
7.94 \\
0.55 \\
0.01 \\
0.30 \\
1.45 \\
3.25\end{array}$ & $\begin{array}{l}0.39 \\
0.15 \\
0.66 \\
7.03 \\
0.55 \\
0.04 \\
0.29 \\
0.32 \\
3.54\end{array}$ \\
\hline $\begin{array}{l}\text { Total } \\
\text { Recovery, } \mathrm{g} \\
\text { Recovery (\% of feed) }\end{array}$ & $\begin{array}{l}11.70 \\
94.89\end{array}$ & $\begin{array}{l}13.16 \\
89.04\end{array}$ & $\begin{array}{l}14.35 \\
96.50\end{array}$ & $\begin{array}{l}11.88 \\
97.06\end{array}$ & $\begin{array}{l}14.73 \\
99.39\end{array}$ & $\begin{array}{l}12.97 \\
87.75\end{array}$ \\
\hline $\begin{array}{l}\text { Resid Ash Content } \\
\text { Conversion on ash-free basis }\end{array}$ & $\begin{array}{r}10.07 \\
5.83\end{array}$ & $\begin{array}{l}10.07 \\
10.71\end{array}$ & $\begin{array}{l}12.76 \\
22.60\end{array}$ & $\begin{array}{r}12.76 \\
9.61\end{array}$ & $\begin{array}{r}8.42 \\
23.23\end{array}$ & $\begin{array}{l}11.69 \\
16.92\end{array}$ \\
\hline $\begin{array}{l}\text { Analysis of } 454^{\circ} \mathrm{C}^{*}\left(850^{\circ} \mathrm{F}\right) \\
\text { Product, wt \% dry } \\
\mathrm{C} \\
\mathrm{H} \\
\mathrm{N} \\
\mathrm{S} \\
\mathrm{O} \text { (by diff) } \\
\text { Ash ( } \mathrm{SO}_{3} \text {-free) }\end{array}$ & $\begin{array}{r}80.52 \\
5.89 \\
0.95 \\
1.02 \\
1.24 \\
10.38 \\
\end{array}$ & $\begin{array}{r}77.71 \\
6.32 \\
0.65 \\
3.11 \\
-1.22 \\
13.42 \\
\end{array}$ & $\begin{array}{r}72.61 \\
6.05 \\
0.64 \\
3.43 \\
-1.37 \\
18.65 \\
\end{array}$ & $\begin{array}{r}77.18 \\
6.09 \\
1.03 \\
1.23 \\
0.65 \\
13.82 \\
\end{array}$ & $\begin{array}{r}78.29 \\
5.86 \\
0.68 \\
2.99 \\
-1.03 \\
13.47\end{array}$ & $\begin{array}{r}73.08 \\
5.60 \\
0.67 \\
3.61 \\
-0.06 \\
17.10\end{array}$ \\
\hline Comments: & & & & & & $\begin{array}{l}\text { ice used for } \\
\text { trap - not } \mathrm{CO}_{2}\end{array}$ \\
\hline $\begin{array}{l}\text { Analysis of Product Gases, } \mathbf{g} \\
\text { methane } \\
\text { ethane } \\
\text { propane } \\
\text { propylene } \\
\text { n-butane } \\
\mathrm{CO} \\
\mathrm{CO}_{2} \\
\mathrm{H}_{2} \mathrm{~S} \\
\mathrm{H}_{2} \\
\text { Total }\end{array}$ & & $\begin{array}{r}0.1502 \\
0.0090 \\
0.0044 \\
0.0005 \\
0.0007 \\
0.0056 \\
0.0165 \\
0.0166 \\
0.1864 \\
0.39\end{array}$ & $\begin{array}{r}0.1656 \\
0.0116 \\
0.0065 \\
0 . \\
0.0015 \\
0.0060 \\
0.0152 \\
0.0486 \\
0.1750 \\
0.43\end{array}$ & $\begin{array}{r}0.0006 \\
0.0016 \\
0.0009 \\
- \\
0.0004 \\
- \\
0.0073 \\
0.0007 \\
0.1185 \\
0.13\end{array}$ & $\begin{array}{r}0.2127 \\
0.0108 \\
0.0057 \\
- \\
- \\
- \\
0.0209 \\
0.0403 \\
0.2195 \\
0.51\end{array}$ & $\begin{array}{r}0.0515 \\
0.0035 \\
0.0015 \\
- \\
- \\
0.2568 \\
0.0052 \\
0.0714 \\
0.39\end{array}$ \\
\hline
\end{tabular}


TABLE 16 (Continued)

RESID REACTIVITY TESTS

\begin{tabular}{|c|c|c|c|c|c|c|}
\hline Run No. & $\begin{array}{l}\text { RR-14 } \\
8 / 29 / 97\end{array}$ & $\begin{array}{l}\text { RR-15 } \\
\text { 9/3/96 }\end{array}$ & $\begin{array}{l}R R-16 \\
9 / 4 / 96\end{array}$ & $\begin{array}{l}\text { RR-17 } \\
\text { 9/5/96 }\end{array}$ & $\begin{array}{l}\text { RR-18 } \\
9 / 6 / 96\end{array}$ & $\begin{array}{l}\text { RR-19 } \\
\text { 9/9/96 }\end{array}$ \\
\hline $\begin{array}{l}\text { Feed } \\
\text { Resid Source } \\
\text { Resid, g } \\
\text { Mo Naphthenate, g } \\
\text { DMDS, g } \\
\text { Tetralin, } \mathrm{g} \\
\mathrm{H}_{2}, \mathrm{~g} \\
\end{array}$ & $\begin{array}{r}\text { W260 R1235 } \\
4.00 \\
2.00 \\
0.50 \\
8.00 \\
0.26 \\
\end{array}$ & $\begin{array}{r}\text { W262 V1067 } \\
4.00 \\
2.00 \\
0.50 \\
8.00 \\
0.28 \\
\end{array}$ & 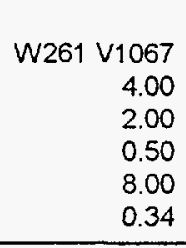 & $\begin{array}{r}W 260 \vee 1067 \\
4.00 \\
2.00 \\
0.50 \\
8.00 \\
0.30 \\
\end{array}$ & $\begin{array}{r}\text { W261 V131B } \\
4.00 \\
2.00 \\
0.50 \\
8.00 \\
0.27 \\
\end{array}$ & $\begin{array}{r}\text { W259 R1235 } \\
4.00 \\
2.00 \\
0.50 \\
8.00 \\
0.28 \\
\end{array}$ \\
\hline $\begin{array}{l}\text { Product } \\
\text { Gas, } \mathrm{g} \\
\text { Distillate } \\
\text { Cold Trap 1, g } \\
\text { Cold Trap 2, } \\
\text { Receiver 1, g } \\
\text { Receiver 2, g } \\
\text { Dist. Overhead, } \mathrm{g} \\
\text { Dist. Column, } \mathrm{g} \\
\text { Reactor Head, } \mathrm{g} \\
\text { Reactor Bottom, } \mathrm{g}\end{array}$ & $\begin{array}{l}0.43 \\
3.19 \\
3.03 \\
7.67 \\
1.02 \\
0.07 \\
0.28 \\
0.30 \\
3.71\end{array}$ & $\begin{array}{l}0.76 \\
\\
2.07 \\
2.01 \\
5.87 \\
0.45 \\
0.02 \\
0.20 \\
0.78 \\
3.21\end{array}$ & $\begin{array}{l}0.32 \\
\\
0.95 \\
0.26 \\
7.84 \\
0.64 \\
0.03 \\
0.57 \\
0.77 \\
3.34\end{array}$ & $\begin{array}{l}0.36 \\
0.90 \\
0.06 \\
8.12 \\
0.60 \\
0.03 \\
0.27 \\
0.34 \\
3.62\end{array}$ & $\begin{array}{l}0.45 \\
0.56 \\
1.12 \\
8.01 \\
0.39 \\
0.03 \\
0.26 \\
0.61 \\
3.45\end{array}$ & $\begin{array}{l}0.46 \\
1.80 \\
0.04 \\
7.35 \\
0.82 \\
0.03 \\
0.22 \\
0.75 \\
3.18\end{array}$ \\
\hline $\begin{array}{l}\text { Total } \\
\text { Recovery, } g \\
\text { Recovery (\% of feed) }\end{array}$ & $\begin{array}{r}19.70 \\
133.47\end{array}$ & $\begin{array}{r}15.37 \\
103.99\end{array}$ & $\begin{array}{l}14.72 \\
99.19\end{array}$ & $\begin{array}{l}14.30 \\
96.62\end{array}$ & $\begin{array}{r}14.88 \\
100.74\end{array}$ & $\begin{array}{l}14.65 \\
99.12\end{array}$ \\
\hline $\begin{array}{l}\text { Resid Ash Content } \\
\text { Conversion on ash-free basis }\end{array}$ & $\begin{array}{l}13.13 \\
15.04\end{array}$ & $\begin{array}{l}13.29 \\
25.65\end{array}$ & $\begin{array}{l}14.74 \\
21.55\end{array}$ & $\begin{array}{l}14.25 \\
15.19\end{array}$ & $\begin{array}{r}9.32 \\
18.42\end{array}$ & $\begin{array}{r}8.61 \\
24.61\end{array}$ \\
\hline 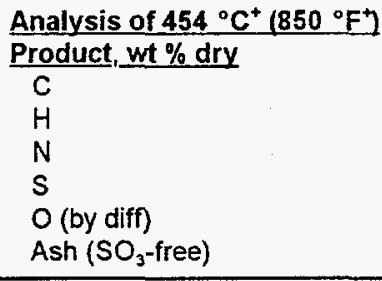 & $\begin{array}{r}71.94 \\
5.48 \\
0.70 \\
3.81 \\
-2.36 \\
20.43 \\
\end{array}$ & $\begin{array}{r}70.5 \\
6.16 \\
0.54 \\
4.08 \\
-0.94 \\
19.66 \\
\end{array}$ & $\begin{array}{r}71.88 \\
6.06 \\
0.57 \\
3.45 \\
-1.86 \\
19.90 \\
\end{array}$ & $\begin{array}{r}70.99 \\
5.50 \\
0.64 \\
3.89 \\
-0.66 \\
19.64 \\
\end{array}$ & $\begin{array}{r}77.28 \\
6.94 \\
0.71 \\
2.76 \\
-1.92 \\
14.23 \\
\end{array}$ & $\begin{array}{r}78.83 \\
5.70 \\
1.00 \\
3.01 \\
-1.27 \\
13.33 \\
\end{array}$ \\
\hline Comments: & & $\begin{array}{l}\text { material lost } \\
\text { during venting }\end{array}$ & & & & \\
\hline $\begin{array}{l}\text { Analysis of Product } \\
\text { Gases, } \mathrm{g} \\
\text { methane } \\
\text { ethane } \\
\text { propane } \\
\text { propylene } \\
\text { n-butane } \\
\mathrm{CO} \\
\mathrm{CO}_{2} \\
\mathrm{H}_{2} \mathrm{~S} \\
\mathrm{H}_{2} \\
\text { Total } \\
\end{array}$ & $\begin{array}{r}0.1147 \\
0.0087 \\
0.0037 \\
- \\
- \\
\\
0.1094 \\
0.0172 \\
0.1769 \\
0.43 \\
\end{array}$ & & $\begin{array}{r}0.0019 \\
0.0090 \\
0.0053 \\
- \\
- \\
- \\
0.0236 \\
0.0193 \\
0.2575 \\
0.32 \\
\end{array}$ & $\begin{array}{r}0.1501 \\
0.0077 \\
0.0037 \\
- \\
- \\
- \\
0.0107 \\
0.0190 \\
0.1688 \\
0.36 \\
\end{array}$ & $\begin{array}{r}0.1870 \\
0.0109 \\
0.006 \\
- \\
0.0018 \\
- \\
0.0121 \\
0.0227 \\
0.2095 \\
0.45 \\
\end{array}$ & $\begin{array}{r}0.1789 \\
0.0102 \\
0.0044 \\
- \\
- \\
0.0123 \\
0.0387 \\
0.0155 \\
0.1999 \\
0.46 \\
\end{array}$ \\
\hline
\end{tabular}


TABLE 16 (Continued)

\section{RESID REACTIVITY TESTS}

\begin{tabular}{|c|c|c|c|c|c|c|}
\hline Run No. & $\begin{array}{l}\text { RR-20 } \\
9 / 10 / 96\end{array}$ & $\begin{array}{l}\text { RR-21 } \\
9 / 11 / 96\end{array}$ & $\begin{array}{l}\text { RR-22 } \\
9 / 12 / 96\end{array}$ & $\begin{array}{l}\text { RR-23 } \\
\text { 9/13/96 }\end{array}$ & $\begin{array}{l}\text { RR-24 } \\
\text { 9/16/96 }\end{array}$ & $\begin{array}{l}\text { RR-9a } \\
\text { 9/17/96 }\end{array}$ \\
\hline $\begin{array}{l}\text { Feed } \\
\text { Resid Source } \\
\text { Resid, } \mathrm{g} \\
\text { Mo Naphthenate, } \mathrm{g} \\
\text { DMDS, } \mathrm{g} \\
\text { Tetralin, } \mathrm{g} \\
\mathrm{H}_{2}, \mathrm{~g}\end{array}$ & $\begin{array}{r}\text { W262 R1235 } \\
4.00 \\
2.00 \\
0.50 \\
8.00 \\
0.26 \\
\end{array}$ & $\begin{array}{r}\text { W262 V131B } \\
4.00 \\
2.00 \\
0.50 \\
8.00 \\
0.26 \\
\end{array}$ & $\begin{array}{r}\text { W258 V131B } \\
4.00 \\
2.00 \\
0.50 \\
8.00 \\
0.25 \\
\end{array}$ & $\begin{array}{r}\text { HRI } 0.43 \\
\text { POC-1 } 57 \mathrm{~B} \\
4.00 \\
2.00 \\
0.50 \\
8.00 \\
0.37 \\
\end{array}$ & $\begin{array}{r}\text { HRI O-43 } \\
\text { POC-02 } \\
4.00 \\
2.00 \\
0.50 \\
8.00 \\
0.33\end{array}$ & $\begin{array}{r}\text { W259 V1067 } \\
4.00 \\
2.00 \\
0.50 \\
8.00 \\
0.26 \\
\end{array}$ \\
\hline $\begin{array}{l}\text { Product } \\
\text { Gas, } \mathrm{g} \\
\text { Distillate } \\
\text { Cold Trap 1, g } \\
\text { Cold Trap } 2, \mathrm{~g} \\
\text { Receiver } 1, \mathrm{~g} \\
\text { Receiver } 2, \mathrm{~g} \\
\text { Dist. Overhead, } \mathrm{g} \\
\text { Dist. Column, } \mathrm{g} \\
\text { Reactor Head, } \mathrm{g} \\
\text { Reactor Bottom, g }\end{array}$ & $\begin{array}{l}1.28 \\
\\
0.32 \\
0.06 \\
8.05 \\
0.62 \\
0.02 \\
0.21 \\
0.90 \\
3.17\end{array}$ & $\begin{array}{l}0.36 \\
0.91 \\
0.10 \\
8.22 \\
0.68 \\
0.03 \\
0.23 \\
0.40 \\
3.63\end{array}$ & $\begin{array}{l}0.57 \\
\\
0.58 \\
0.13 \\
7.88 \\
0.67 \\
0.02 \\
0.25 \\
0.37 \\
3.74\end{array}$ & $\begin{array}{l}0.43 \\
0.52 \\
0.37 \\
8.02 \\
0.96 \\
0.09 \\
0.22 \\
0.18 \\
3.46\end{array}$ & $\begin{array}{l}0.48 \\
\\
0.71 \\
0.36 \\
8.17 \\
0.69 \\
0.04 \\
0.22 \\
0.38 \\
3.59\end{array}$ & $\begin{array}{l}0.43 \\
0.49 \\
0.13 \\
8.38 \\
0.72 \\
0.03 \\
0.25 \\
0.31 \\
3.72\end{array}$ \\
\hline $\begin{array}{l}\text { Total } \\
\text { Recovery, } \mathrm{g} \\
\text { Recovery ( } \% \text { of feed) }\end{array}$ & $\begin{array}{l}14.63 \\
99.12\end{array}$ & $\begin{array}{l}14.56 \\
98.64\end{array}$ & $\begin{array}{l}14.21 \\
96.34\end{array}$ & $\begin{array}{l}14.25 \\
95.83\end{array}$ & $\begin{array}{l}14.64 \\
98.72\end{array}$ & $\begin{array}{l}14.46 \\
97.97\end{array}$ \\
\hline $\begin{array}{l}\text { Resid Ash Content } \\
\text { Conversion on ash-free basis }\end{array}$ & $\begin{array}{l}11.94 \\
26.97\end{array}$ & $\begin{array}{l}12.23 \\
15.04\end{array}$ & $\begin{array}{l}13.71 \\
11.92\end{array}$ & $\begin{array}{r}0.4 \\
17.49\end{array}$ & $\begin{array}{r}4.09 \\
14.82\end{array}$ & $\begin{array}{l}10.07 \\
11.63\end{array}$ \\
\hline $\begin{array}{l}\text { Analysis of } 454^{\circ} \mathrm{C}^{+}\left(850^{\circ} \mathrm{F}^{+}\right) \\
\text {Product, wt \% dry } \\
\mathrm{C} \\
\mathrm{H} \\
\mathrm{N} \\
\mathrm{S} \\
\mathrm{O} \text { (by diff) } \\
\text { Ash (SO } \mathrm{SO}_{3} \text {-free) }\end{array}$ & $\begin{array}{r}70.4 \\
6.49 \\
0.57 \\
4.20 \\
-0.51 \\
18.85 \\
\end{array}$ & $\begin{array}{r}72.13 \\
6.45 \\
0.57 \\
3.87 \\
-0.85 \\
17.83 \\
\end{array}$ & $\begin{array}{r}72.01 \\
4.88 \\
0.82 \\
3.59 \\
0.00 \\
18.71 \\
\end{array}$ & $\begin{array}{r}85.3 \\
9.34 \\
0.42 \\
2.10 \\
-2.16 \\
5 \\
\end{array}$ & $\begin{array}{r}82.45 \\
8.32 \\
0.38 \\
2.19 \\
-2.31 \\
8.97\end{array}$ & $\begin{array}{r}76.92 \\
5.78 \\
0.07 \\
3.07 \\
-1.02 \\
14.55\end{array}$ \\
\hline Comments: & $\begin{array}{l}\text { material lost } \\
\text { during venting }\end{array}$ & & & & & \\
\hline $\begin{array}{l}\text { Analysis of Product Gases, } \mathrm{g} \\
\text { methane } \\
\text { ethane } \\
\text { propane } \\
\text { propylene } \\
\text { n-butane } \\
\mathrm{CO} \\
\mathrm{CO}_{2} \\
\mathrm{H}_{2} \mathrm{~S} \\
\mathrm{H}_{2} \\
\text { Total }\end{array}$ & & $\begin{array}{r}0.0275 \\
0.0008 \\
0.0012 \\
0.0006 \\
0.0817 \\
0.2025 \\
0.0039 \\
0.0418 \\
0.36\end{array}$ & $\begin{array}{r}0.1491 \\
0.0093 \\
0.0037 \\
- \\
0.0246 \\
0.0317 \\
0.0758 \\
0.0241 \\
0.2516 \\
0.57\end{array}$ & $\begin{array}{r}0.0511 \\
0.0030 \\
0.0014 \\
- \\
0.0068 \\
0.101 \\
0.1851 \\
0.009 \\
0.0726 \\
0.43\end{array}$ & $\begin{array}{r}0.0739 \\
0.0045 \\
0.0221 \\
- \\
0.0146 \\
0.0732 \\
0.1267 \\
0.0160 \\
0.1490 \\
0.48\end{array}$ & \\
\hline
\end{tabular}


TABLE 16 (Continued)

RESID REACTIVITY TESTS

\begin{tabular}{|c|c|c|c|c|c|c|}
\hline Run No. & $\begin{array}{l}R R-13 a \\
9 / 18 / 96\end{array}$ & $\begin{array}{l}\text { RR-14a } \\
\text { 9/19/96 }\end{array}$ & $\begin{array}{l}\text { RR-17a } \\
\text { g/20/96 }\end{array}$ & $\begin{array}{l}\text { RR-22a } \\
\text { 9/23/96 }\end{array}$ & $\begin{array}{l}\text { RR-25 } \\
\text { 9/24/96 }\end{array}$ & $\begin{array}{l}\text { RR-26 } \\
\text { 9/25/96 }\end{array}$ \\
\hline $\begin{array}{l}\text { Feed } \\
\text { Resid Source } \\
\text { Resid, } g \\
\text { Mo Naphthenate, } g \\
\text { DMDS, } g \\
\text { Tetralin, } g \\
\mathrm{H}_{2}, \mathrm{~g}\end{array}$ & $\begin{array}{r}W 260 \vee 1318 \\
4.00 \\
2.00 \\
0.50 \\
8.00 \\
0.23 \\
\end{array}$ & $\begin{array}{r}\text { W260 R1235 } \\
4.00 \\
2.00 \\
0.50 \\
8.00 \\
0.31 \\
\end{array}$ & $\begin{array}{r}W 260 \vee 1067 \\
4.00 \\
2.00 \\
0.50 \\
8.00 \\
0.26 \\
\end{array}$ & $\begin{array}{r}W 258 \mathrm{~V} 131 \mathrm{~B} \\
4.00 \\
2.00 \\
0.50 \\
8.00 \\
0.26 \\
\end{array}$ & $\begin{array}{r}\text { W259 R1235 } \\
4.00 \\
- \\
- \\
8.00 \\
0.27 \\
\end{array}$ & $\begin{array}{r}\text { W260 R1235 } \\
4.00 \\
- \\
- \\
8.00 \\
0.27\end{array}$ \\
\hline $\begin{array}{l}\text { Product } \\
\text { Gas, } \mathrm{g} \\
\text { Distillate } \\
\text { Cold Trap 1, g } \\
\text { Cold Trap 2, g } \\
\text { Receiver } 1, \mathrm{~g} \\
\text { Receiver 2, } \\
\text { Dist. Overhead, g } \\
\text { Dist. Column, g } \\
\text { Reactor Head, g } \\
\text { Reactor Bottom, g }\end{array}$ & $\begin{array}{r}0.28 \\
0.45 \\
0.31 \\
7.99 \\
0.79 \\
0.04 \\
0.2 \\
0.3 \\
3.65\end{array}$ & $\begin{array}{r}0.39 \\
1.14 \\
0.29 \\
7.62 \\
0.85 \\
0.04 \\
0.28 \\
0.58 \\
3.3\end{array}$ & $\begin{array}{r}0.33 \\
0.69 \\
0.34 \\
8.06 \\
0.76 \\
0.04 \\
0.2 \\
0.34 \\
3.66\end{array}$ & $\begin{array}{r}0.35 \\
0.95 \\
0.35 \\
7.74 \\
0.8 \\
0.04 \\
0.18 \\
0.39 \\
3.76\end{array}$ & $\begin{array}{r}0.23 \\
0.58 \\
0.25 \\
7.2 \\
0.04 \\
0.03 \\
0.09 \\
0.24 \\
3.73\end{array}$ & $\begin{array}{l}0.29 \\
\\
0.9 \\
0.14 \\
7.07 \\
0.01 \\
0.03 \\
0.08 \\
0.16 \\
3.67\end{array}$ \\
\hline $\begin{array}{l}\text { Total } \\
\text { Recovery, } \mathrm{g} \\
\text { Recovery (\% of feed) }\end{array}$ & $\begin{array}{l}14.01 \\
95.11\end{array}$ & $\begin{array}{l}14.49 \\
97.84\end{array}$ & $\begin{array}{l}14.42 \\
97.70\end{array}$ & $\begin{array}{l}14.56 \\
98.64\end{array}$ & $\begin{array}{r}12.39 \\
100.98\end{array}$ & $\begin{array}{r}12.35 \\
100.65\end{array}$ \\
\hline $\begin{array}{l}\text { Resid Ash Content } \\
\text { Conversion on ash-free basis }\end{array}$ & $\begin{array}{l}11.69 \\
14.57\end{array}$ & $\begin{array}{l}13.13 \\
22.21\end{array}$ & $\begin{array}{l}14.25 \\
14.42\end{array}$ & $\begin{array}{l}13.71 \\
11.65\end{array}$ & $\begin{array}{l}8.61 \\
7.78\end{array}$ & $\begin{array}{l}13.13 \\
12.26\end{array}$ \\
\hline $\begin{array}{l}\text { Analysis of } 454^{\circ} \mathrm{C}^{+}\left(850^{\circ} \mathrm{F}\right) \\
\text { Product, wt \% dry } \\
\mathrm{C} \\
\mathrm{H} \\
\mathrm{N} \\
\mathrm{S} \\
\mathrm{O} \text { (by diff) } \\
\text { Ash ( } \mathrm{SO}_{3} \text {-free) }\end{array}$ & $\begin{array}{r}73.16 \\
5.92 \\
0.67 \\
3.66 \\
-0.73 \\
17.32 \\
\end{array}$ & $\begin{array}{r}70.13 \\
5.38 \\
0.65 \\
4.53 \\
1.22 \\
18.09 \\
\end{array}$ & $\begin{array}{r}70.92 \\
5.46 \\
0.68 \\
4.11 \\
-0.97 \\
19.8 \\
\end{array}$ & $\begin{array}{r}72.46 \\
5.15 \\
0.77 \\
3.72 \\
-0.99 \\
18.9 \\
\end{array}$ & $\begin{array}{r}81.63 \\
5.90 \\
1.02 \\
1.42 \\
0.41 \\
9.62\end{array}$ & $\begin{array}{r}75.68 \\
5.78 \\
0.99 \\
1.73 \\
-1.11 \\
16.93\end{array}$ \\
\hline Comments: & & & & & & \\
\hline $\begin{array}{l}\text { Analysis of Product } \\
\text { Gases, } \mathrm{g} \\
\text { methane } \\
\text { ethane } \\
\text { propane } \\
\text { propylene } \\
\text { n-butane } \\
\mathrm{CO} \\
\mathrm{CO}_{2} \\
\mathrm{H}_{2} \mathrm{~S} \\
\mathrm{H}_{2} \\
\text { Total }\end{array}$ & & & & & $\begin{array}{r}0 \\
0.0078 \\
0.0063 \\
- \\
0.0041 \\
- \\
0.0084 \\
0 \\
0.2034 \\
0.23\end{array}$ & $\begin{array}{r}0.0054 \\
0.0038 \\
0.0018 \\
- \\
- \\
0.0062 \\
0.0184 \\
0 \\
0.2545 \\
0.29\end{array}$ \\
\hline
\end{tabular}


TABLE 16 (Continued)

RESID REACTIVITY TESTS

\begin{tabular}{|c|c|c|c|c|c|c|}
\hline Run No. & $\begin{array}{l}\text { RR-27 } \\
\text { 9/26/96 }\end{array}$ & $\begin{array}{l}\text { RR-28 } \\
\text { 9/27/96 }\end{array}$ & $\begin{array}{l}\text { RR-29 } \\
\text { 9/28/96 }\end{array}$ & $\begin{array}{l}R R-30 \\
10 / 1 / 96\end{array}$ & $\begin{array}{l}\text { RR-31 } \\
10 / 2 / 96\end{array}$ & $\begin{array}{l}\text { RR-32 } \\
10 / 3 / 96\end{array}$ \\
\hline $\begin{array}{l}\text { Feed } \\
\text { Resid Source } \\
\text { Resid, } g \\
\text { Mo Naphthenate, } g \\
\text { DMDS, } g \\
\text { Tetralin, } g \\
\mathrm{H}_{2}, \mathrm{~g}\end{array}$ & $\begin{array}{r}\text { W261 R1235 } \\
4.00 \\
- \\
- \\
8.00 \\
0.29\end{array}$ & $\begin{array}{r}W 262 R 1235 \\
4.00 \\
- \\
- \\
8.00 \\
0.27\end{array}$ & $\begin{array}{r}\text { HRI O-43 } \\
\text { POC-1 } 57 \mathrm{~B} \\
4.00 \\
- \\
- \\
8.00 \\
0.25\end{array}$ & $\begin{array}{r}\text { W59 V131B } \\
4.00 \\
- \\
- \\
8.00 \\
0.26\end{array}$ & $\begin{array}{r}W 260 \vee 1318 \\
4.00 \\
- \\
- \\
8.00 \\
0.25\end{array}$ & $\begin{array}{r}W 260 V 131 B \\
4.00 \\
- \\
8.00 \\
0.27\end{array}$ \\
\hline $\begin{array}{l}\text { Product } \\
\text { Gas, } \mathrm{g} \\
\text { Distillate } \\
\text { Cold Trap 1, g } \\
\text { Cold Trap 2, } \\
\text { Receiver 1, } \mathrm{g} \\
\text { Receiver 2, } \\
\text { Dist. Overhead, g } \\
\text { Dist. Column, } \mathrm{g} \\
\text { Reactor Head, g } \\
\text { Reactor Bottom, g }\end{array}$ & $\begin{array}{r}0.16 \\
0.28 \\
0.3 \\
7.05 \\
0.02 \\
0.01 \\
0.03 \\
0.38 \\
3.66\end{array}$ & $\begin{array}{r}0.18 \\
\\
0.33 \\
0.23 \\
7.38 \\
0.02 \\
0.01 \\
0.1 \\
0.23 \\
3.64\end{array}$ & $\begin{array}{l}0.21 \\
\\
0.77 \\
0.19 \\
7.25 \\
0.01 \\
0.01 \\
0.06 \\
0.12 \\
3.72\end{array}$ & $\begin{array}{r}0.18 \\
\\
0.03 \\
0.03 \\
7.93 \\
0.03 \\
0 \\
0 \\
0.2 \\
3.78\end{array}$ & $\begin{array}{r}0.21 \\
\\
0.02 \\
0.06 \\
7.85 \\
0.04 \\
0 \\
0.1 \\
0.1 \\
3.66\end{array}$ & $\begin{array}{r}0.29 \\
\\
0.46 \\
0.08 \\
7.3 \\
0.01 \\
0 \\
0.06 \\
0.09 \\
3.79\end{array}$ \\
\hline $\begin{array}{l}\text { Total } \\
\text { Recovery, } \mathrm{g} \\
\text { Recovery ( } \% \text { of feed) }\end{array}$ & $\begin{array}{l}11.89 \\
96.75\end{array}$ & $\begin{array}{l}12.12 \\
98.78\end{array}$ & $\begin{array}{r}12.34 \\
100.73\end{array}$ & $\begin{array}{l}12.18 \\
99.35\end{array}$ & $\begin{array}{l}12.04 \\
98.29\end{array}$ & $\begin{array}{l}12.08 \\
98.45\end{array}$ \\
\hline $\begin{array}{l}\text { Resid Ash Content } \\
\text { Conversion on ash-free basis }\end{array}$ & $\begin{array}{r}12.76 \\
9.07\end{array}$ & $\begin{array}{l}11.94 \\
10.10\end{array}$ & $\begin{array}{r}0.4 \\
7.00\end{array}$ & $\begin{array}{l}8.42 \\
5.71\end{array}$ & $\begin{array}{r}11.69 \\
9.02\end{array}$ & $\begin{array}{l}9.32 \\
5.64\end{array}$ \\
\hline $\begin{array}{l}\text { Analysis of } 454^{\circ} \mathrm{C}^{+}\left(850^{\circ} \mathrm{F}\right) \\
\text { Product, wt \% dry } \\
\mathrm{C} \\
\mathrm{H} \\
\mathrm{N} \\
\mathrm{S} \\
\mathrm{O} \text { (by diff) } \\
\text { Ash ( } \mathrm{SO}_{3} \text {-free) }\end{array}$ & $\begin{array}{r}77.76 \\
6.36 \\
1.02 \\
1.28 \\
0.28 \\
13.30 \\
\end{array}$ & $\begin{array}{r}76.28 \\
6.60 \\
0.90 \\
1.72 \\
14.5 \\
13.00 \\
\end{array}$ & $\begin{array}{r}86.73 \\
9.79 \\
0.80 \\
0.29 \\
1.99 \\
0.40 \\
\end{array}$ & $\begin{array}{r}83.47 \\
5.84 \\
1.17 \\
1.15 \\
-0.25 \\
8.62 \\
\end{array}$ & $\begin{array}{r}77.39 \\
5.58 \\
0.90 \\
1.53 \\
2.41 \\
12.19 \\
\end{array}$ & $\begin{array}{r}80.69 \\
6.44 \\
0.81 \\
0.92 \\
1.45 \\
9.69 \\
\end{array}$ \\
\hline Comments: & & & & & & \\
\hline $\begin{array}{l}\text { Analysis of Product } \\
\text { Gases, } \mathrm{g} \\
\text { methane } \\
\text { ethane } \\
\text { propane } \\
\text { propylene } \\
\text { n-butane } \\
\mathrm{CO} \\
\mathrm{CO}_{2} \\
\mathrm{H}_{2} \mathrm{~S} \\
\mathrm{H}_{2} \\
\text { Total }\end{array}$ & $\begin{array}{r}0.0014 \\
0.0023 \\
0.0010 \\
- \\
0.0015 \\
0.0077 \\
0.0004 \\
0.1458 \\
0.16 \\
\end{array}$ & $\begin{array}{r}0.0011 \\
0.0027 \\
0.0010 \\
- \\
- \\
- \\
0.0055 \\
0 \\
0.1697 \\
0.18\end{array}$ & $\begin{array}{r}0.0004 \\
0.0018 \\
0.0011 \\
- \\
- \\
0.0011 \\
0 \\
0.2056 \\
0.21\end{array}$ & $\begin{array}{r}0.0008 \\
0.0018 \\
0.0010 \\
- \\
- \\
- \\
0.0021 \\
0 \\
0.1744 \\
0.18 \\
\end{array}$ & $\begin{array}{r}0.0020 \\
0.0031 \\
0.0015 \\
- \\
- \\
0.0025 \\
0.0055 \\
0 \\
0.1954 \\
0.21 \\
\end{array}$ & $\begin{array}{r}0.0035 \\
0.0035 \\
0.0022 \\
- \\
- \\
0.0028 \\
0.0074 \\
0 \\
0.2705 \\
0.29 \\
\end{array}$ \\
\hline
\end{tabular}


TABLE 16 (Continued)

RESID REACTIVITY TESTS

\begin{tabular}{|c|c|c|c|c|c|c|}
\hline Run No. & $\begin{array}{l}\text { RR.33 } \\
10 / 4 / 96\end{array}$ & $\begin{array}{l}\text { RR-34 } \\
\text { 10/7/96 }\end{array}$ & $\begin{array}{l}\text { RR-35 } \\
10 / 8 / 96\end{array}$ & RR-36 & $\begin{array}{c}\text { RR-37 } \\
10 / 22 / 96\end{array}$ & $\begin{array}{c}\text { RR-38 } \\
10 / 23 / 96\end{array}$ \\
\hline $\begin{array}{l}\text { Feed } \\
\text { Resid Source } \\
\text { Resid, } g \\
\text { Mo Naphthenate, } g \\
\text { DMDS, } g \\
\text { Tetralin, } g \\
\mathrm{H}_{2}, \mathrm{~g}\end{array}$ & $\begin{array}{r}\text { W262 V131B } \\
4.00 \\
- \\
- \\
8.00 \\
0.27\end{array}$ & $\begin{array}{r}\text { HRI } 0-43 \\
\text { POC-2 } \\
4.00 \\
- \\
- \\
8.00 \\
0.28 \\
\end{array}$ & $\begin{array}{r}W 259 \text { V1067 } \\
4.00 \\
- \\
8.00 \\
0.28\end{array}$ & $\begin{array}{r}\text { W260 V1067 } \\
4.00 \\
- \\
- \\
8.00 \\
0.26\end{array}$ & $\begin{array}{r}\text { W261 V1067 } \\
4.00 \\
- \\
- \\
8.00 \\
0.25\end{array}$ & $\begin{array}{r}\text { W262 V1067 } \\
4.00 \\
- \\
- \\
8.00 \\
0.27\end{array}$ \\
\hline $\begin{array}{l}\text { Product } \\
\text { Gas, } \mathrm{g} \\
\text { Distillate } \\
\text { Cold Trap 1,g } \\
\text { Cold Trap 2, } \\
\text { Receiver } 1, \mathrm{~g} \\
\text { Receiver 2, } \\
\text { Dist. Overhead, } \mathrm{g} \\
\text { Dist. Column, } \\
\text { Reactor Head, } \mathrm{g} \\
\text { Reactor Bottom, g }\end{array}$ & $\begin{array}{r}0.17 \\
0.5 \\
0.05 \\
7.53 \\
0.01 \\
0.01 \\
0.01 \\
0.16 \\
3.71\end{array}$ & $\begin{array}{r}0.25 \\
0.21 \\
0.1 \\
7.69 \\
0.14 \\
0 \\
0.07 \\
0.11 \\
3.8\end{array}$ & $\begin{array}{l}0.27 \\
\\
0.46 \\
0.08 \\
7.53 \\
0.02 \\
0.01 \\
0.05 \\
0.11 \\
3.76\end{array}$ & $\begin{array}{r}0.18 \\
\\
0.62 \\
0.12 \\
7.44 \\
0.02 \\
0 \\
0.15 \\
0.05 \\
3.64\end{array}$ & $\begin{array}{l}0.25 \\
\\
0.64 \\
0.15 \\
7.27 \\
0.01 \\
0.01 \\
0.02 \\
0.07 \\
3.75\end{array}$ & $\begin{array}{r}0.21 \\
0.65 \\
0.12 \\
7.43 \\
0.03 \\
0 \\
0.03 \\
0.1 \\
3.71\end{array}$ \\
\hline $\begin{array}{l}\text { Total } \\
\text { Recovery, } \mathbf{g} \\
\text { Recovery (\% of feed) }\end{array}$ & $\begin{array}{l}12.15 \\
99.02\end{array}$ & $\begin{array}{r}12.37 \\
100.73\end{array}$ & $\begin{array}{r}12.29 \\
100.08\end{array}$ & $\begin{array}{l}12.22 \\
99.67\end{array}$ & $\begin{array}{l}12.17 \\
99.35\end{array}$ & $\begin{array}{r}12.28 \\
100.08\end{array}$ \\
\hline $\begin{array}{l}\text { Resid Ash Content } \\
\text { Conversion on ash-free basis }\end{array}$ & $\begin{array}{r}12.23 \\
7.86\end{array}$ & $\begin{array}{l}4.09 \\
5.16\end{array}$ & $\begin{array}{r}10.07 \\
6.27\end{array}$ & $\begin{array}{r}14.25 \\
9.46\end{array}$ & $\begin{array}{r}14.74 \\
6.78\end{array}$ & $\begin{array}{r}13.29 \\
8.81\end{array}$ \\
\hline $\begin{array}{l}\text { Analysis of } 454^{\circ} \mathrm{C}^{+}\left(850^{\circ} \mathrm{F}^{+}\right) \\
\text {Product, wt \% dry } \\
\mathrm{C} \\
\mathrm{H} \\
\mathrm{N} \\
\mathrm{S} \\
\mathrm{O} \text { (by diff) } \\
\text { Ash ( } \mathrm{SO}_{3} \text {-free) }\end{array}$ & $\begin{array}{r}76.39 \\
5.95 \\
0.91 \\
1.59 \\
2.34 \\
12.81\end{array}$ & $\begin{array}{r}81.73 \\
11.65 \\
0.43 \\
0.20 \\
1.74 \\
4.25\end{array}$ & $\begin{array}{r}81.18 \\
5.40 \\
0.91 \\
1.32 \\
0.86 \\
10.33\end{array}$ & $\begin{array}{r}74.98 \\
5.15 \\
0.93 \\
1.74 \\
2.52 \\
14.68\end{array}$ & $\begin{array}{r}75.63 \\
6.05 \\
0.94 \\
1.25 \\
0.91 \\
15.22\end{array}$ & $\begin{array}{r}74.57 \\
6.04 \\
0.85 \\
1.72 \\
2.07 \\
14.75\end{array}$ \\
\hline Comments: & & & & & & \\
\hline $\begin{array}{l}\text { Analysis of Product } \\
\text { Gases, } \mathrm{g} \\
\text { methane } \\
\text { ethane } \\
\text { propane } \\
\text { propylene } \\
\text { n-butane } \\
\mathrm{CO} \\
\mathrm{CO}_{2} \\
\mathrm{H}_{2} \mathrm{~S} \\
\mathrm{H}_{2} \\
\text { Total } \\
\end{array}$ & $\begin{array}{r}0.0026 \\
0.0029 \\
0.0014 \\
- \\
- \\
0.0045 \\
0.0078 \\
0 \\
0.2508 \\
0.27 \\
\end{array}$ & $\begin{array}{r}0 \\
0.0024 \\
- \\
- \\
- \\
- \\
0.0036 \\
0 \\
0.2440 \\
0.25 \\
\end{array}$ & & $\begin{array}{r}0.0019 \\
0.0028 \\
0.0016 \\
- \\
- \\
0.0023 \\
0.0049 \\
0 \\
0.1663 \\
0.18 \\
\end{array}$ & & \\
\hline
\end{tabular}


TABLE 16 (Continued)

RESID REACTIVITY TESTS

\begin{tabular}{|c|c|c|c|c|c|c|}
\hline Run No. & $\begin{array}{c}\text { RR-39 } \\
10 / 10 / 96\end{array}$ & $\begin{array}{l}\text { RR-13B } \\
10 / 24 / 96\end{array}$ & $\begin{array}{l}\text { RR-14B } \\
10 / 25 / 96\end{array}$ & $\begin{array}{l}\text { RR-22B } \\
10 / 30 / 96\end{array}$ & $\begin{array}{l}\text { RR-14C } \\
10 / 29 / 96\end{array}$ & $\begin{array}{l}\text { RR-26A } \\
10 / 31 / 96\end{array}$ \\
\hline $\begin{array}{l}\text { Feed } \\
\text { Resid Source } \\
\text { Resid, } g \\
\text { Mo Naphthenate, g } \\
\text { DMDS, } g \\
\text { Tetralin, } g \\
\mathrm{H}_{2}, \mathrm{~g}\end{array}$ & $\begin{array}{r}\text { W258 V131B } \\
4.00 \\
- \\
8.00 \\
0.27\end{array}$ & $\begin{array}{r}W 260 \text { V131B } \\
4.00 \\
2.00 \\
0.50 \\
8.00 \\
0.27\end{array}$ & $\begin{array}{r}W 260 \text { V1235 } \\
4.00 \\
2.00 \\
0.50 \\
8.00 \\
0.26\end{array}$ & $\begin{array}{r}\text { W258 V131B } \\
4.00 \\
2.00 \\
0.50 \\
8.00 \\
0.26\end{array}$ & $\begin{array}{r}\text { W260 R1235 } \\
4.00 \\
2.00 \\
0.50 \\
8.00 \\
0.28\end{array}$ & $\begin{array}{r}W 260 R 1235 \\
4.00 \\
- \\
8.00 \\
0.27\end{array}$ \\
\hline $\begin{array}{l}\text { Product } \\
\text { Gas, } \mathrm{g} \\
\text { Distillate } \\
\text { Cold Trap 1, g } \\
\text { Cold Trap 2, g } \\
\text { Receiver 1,g } \\
\text { Receiver 2, g } \\
\text { Dist. Overhead, g } \\
\text { Dist. Column, g } \\
\text { Reactor Head, g } \\
\text { Reactor Bottom, g }\end{array}$ & $\begin{array}{l}0.25 \\
0.65 \\
0.15 \\
7.19 \\
0.01 \\
0.01 \\
0.04 \\
0.11 \\
3.8\end{array}$ & $\begin{array}{r}0.38 \\
\\
0.69 \\
0.14 \\
8.27 \\
0.75 \\
0.01 \\
0.31 \\
0.1 \\
3.67\end{array}$ & $\begin{array}{r}0.35 \\
\\
0.87 \\
0.25 \\
7.83 \\
0.88 \\
0 \\
0.26 \\
0.12 \\
3.67\end{array}$ & $\begin{array}{r}0.49 \\
0.88 \\
0.26 \\
7.69 \\
0.8 \\
0.01 \\
0.26 \\
0.12 \\
3.76\end{array}$ & $\begin{array}{l}0.37 \\
\\
0.47 \\
0.16 \\
8.67 \\
0.86 \\
0.01 \\
0.23 \\
0.13 \\
3.68\end{array}$ & $\begin{array}{r}0.31 \\
\\
0.17 \\
0.15 \\
7.86 \\
0.02 \\
0 \\
0.02 \\
0.06 \\
3.71\end{array}$ \\
\hline $\begin{array}{l}\text { Total } \\
\text { Recovery, } g \\
\text { Recovery (\% of feed) }\end{array}$ & $\begin{array}{l}12.21 \\
99.51\end{array}$ & $\begin{array}{l}14.32 \\
96.95\end{array}$ & $\begin{array}{l}14.23 \\
96.41\end{array}$ & $\begin{array}{l}14.27 \\
96.68\end{array}$ & $\begin{array}{l}14.58 \\
98.65\end{array}$ & $\begin{array}{r}12.30 \\
100.24\end{array}$ \\
\hline $\begin{array}{l}\text { Resid Ash Content } \\
\text { Conversion on ash-free basis }\end{array}$ & $\begin{array}{r}13.71 \\
5.58\end{array}$ & $\begin{array}{l}11.69 \\
13.86\end{array}$ & $\begin{array}{l}13.13 \\
15.17\end{array}$ & $\begin{array}{l}13.71 \\
11.19\end{array}$ & $\begin{array}{l}13.13 \\
14.04\end{array}$ & $\begin{array}{r}13.13 \\
7.74\end{array}$ \\
\hline $\begin{array}{l}\text { Analysis of } 454^{\circ} \mathrm{C}^{+}\left(850^{\circ} \mathrm{F}\right) \\
\text { Product wh \% dry } \\
\mathrm{C} \\
\mathrm{H} \\
\mathrm{N} \\
\mathrm{S} \\
\mathrm{O} \text { (by diff) } \\
\text { Ash ( } \mathrm{SO}_{3} \text {-free) }\end{array}$ & $\begin{array}{r}76.49 \\
4.91 \\
0.98 \\
1.57 \\
1.81 \\
14.24\end{array}$ & $\begin{array}{r}73.43 \\
5.7 \\
0.65 \\
3.67 \\
-0.54 \\
17.09\end{array}$ & $\begin{array}{r}71.32 \\
5.34 \\
0.66 \\
3.97 \\
-0.96 \\
19.68\end{array}$ & $\begin{array}{r}72.51 \\
5.15 \\
0.74 \\
3.73 \\
-0.59 \\
18.47\end{array}$ & $\begin{array}{r}71.91 \\
5.45 \\
0.73 \\
3.83 \\
-0.75 \\
18.83\end{array}$ & $\begin{array}{r}75.35 \\
5.42 \\
0.95 \\
1.45 \\
3.24 \\
13.59\end{array}$ \\
\hline Comments: & & & & & & \\
\hline $\begin{array}{l}\text { Analysis of Product } \\
\text { Gases, } \mathrm{g} \\
\text { methane } \\
\text { ethane } \\
\text { propane } \\
\text { propylene } \\
\text { n-butane } \\
\mathrm{CO} \\
\mathrm{CO} \\
\mathrm{H}_{2} \mathrm{~S} \\
\mathrm{H}_{2} \\
\text { Total } \\
\end{array}$ & $\begin{array}{r}0.0002 \\
0.0024 \\
0.0014 \\
- \\
- \\
0.0013 \\
0.0042 \\
0 \\
0.2404 \\
0.25 \\
\end{array}$ & $\begin{array}{r}0.1424 \\
0.0086 \\
0.0036 \\
- \\
- \\
- \\
0.0108 \\
0.0196 \\
0.1950 \\
0.38 \\
\end{array}$ & $\begin{array}{r}0.1092 \\
0.0083 \\
0.0038 \\
- \\
- \\
0.0012 \\
0.0103 \\
0.0145 \\
0.2026 \\
0.35 \\
\end{array}$ & $\begin{array}{r}0.1571 \\
0.0171 \\
0.0053 \\
- \\
- \\
\\
0.0105 \\
0.0204 \\
0.2795 \\
0.49 \\
\end{array}$ & $\begin{array}{r}0.1270 \\
0.0081 \\
0.0040 \\
- \\
- \\
0.0050 \\
0.0119 \\
0.0123 \\
0.2017 \\
0.37 \\
\end{array}$ & $\begin{array}{r}- \\
- \\
- \\
- \\
- \\
0.0111 \\
0.2989 \\
0.31 \\
\end{array}$ \\
\hline
\end{tabular}


TABLE 17

COMPARISON OF RESID CONVERSION DATA OBTAINED

IN $45 \mathrm{~mL}$ MICROAUTOCLAVES (CONSOL R\&D) AND

SHORT TIME BATCH REACTOR (UNIVERSITY OF DELAWARE)

\begin{tabular}{||c|r|r|r|r||}
\hline \multirow{2}{*}{ Resid } & \multicolumn{2}{|c|}{ UOD Conversion } & \multicolumn{2}{c|}{ CONSOL Conversion } \\
\cline { 2 - 5 } & Thermal & Catalytic & \multicolumn{1}{c|}{ Thermal } & Catalytic \\
\hline W258, V131B & 16.1 & 35.3 & 5.6 & 11.9 \\
\hline W259, V1067 & 14.4 & 40.8 & 5.8 & 10.7 \\
W259, R1235 & 15.3 & 36.9 & 7.8 & 24.6 \\
W259, V131B & 15.8 & 31.7 & 5.7 & 23.2 \\
\hline W260, V1067 & 18.1 & & 9.5 & 15.2 \\
W260, R1235 & 18.4 & 33.5 & 12.3 & 15.0 \\
W260, V131B & 21.8 & 44.9 & 9.0 & 16.9 \\
\hline W261, V1067 & 21.1 & 43.0 & 6.8 & 21.6 \\
W261, R1235 & 15.4 & & 9.6 & 22.6 \\
W261, V131B & 16.3 & 34.4 & 5.6 & 18.4 \\
\hline W262, V1067 & 15.8 & 36.2 & 8.8 & 25.7 \\
W262, R1235 & 17.8 & 30.2 & 10.1 & 27.0 \\
W262, V131B & 18.7 & 34.1 & 7.9 & 15.0 \\
\hline HRI POC10-43 & & & 7.0 & 17.5 \\
HRI POC2 O-43 & & & 5.2 & 14.8 \\
\hline
\end{tabular}


TABLE 18

GAS CHROMATOGRAPHY CALIBRATION GASES

\begin{tabular}{||l|c|}
\hline \multicolumn{1}{|c|}{ Component } & Volume, $\%$ \\
\hline methane & 8.0 \\
ethane & 3.0 \\
ethylene & 0.5 \\
propane & 2.0 \\
propylene & 0.5 \\
n-butane & 1.0 \\
l-butane & 0.5 \\
1-butene & 0.5 \\
trans-2-butene & 0.5 \\
cis-2-butene & 0.5 \\
n-pentane & 0.5 \\
I-pentane & 0.5 \\
carbon monoxide & 1.0 \\
carbon dioxide & 1.0 \\
nitrogen & 0.5 \\
argon & 1.0 \\
hydrogen & 78.5 \\
\hline
\end{tabular}




\section{HTI Run ALC-1 \\ Component Distribution Of Feed Slurry Samples}

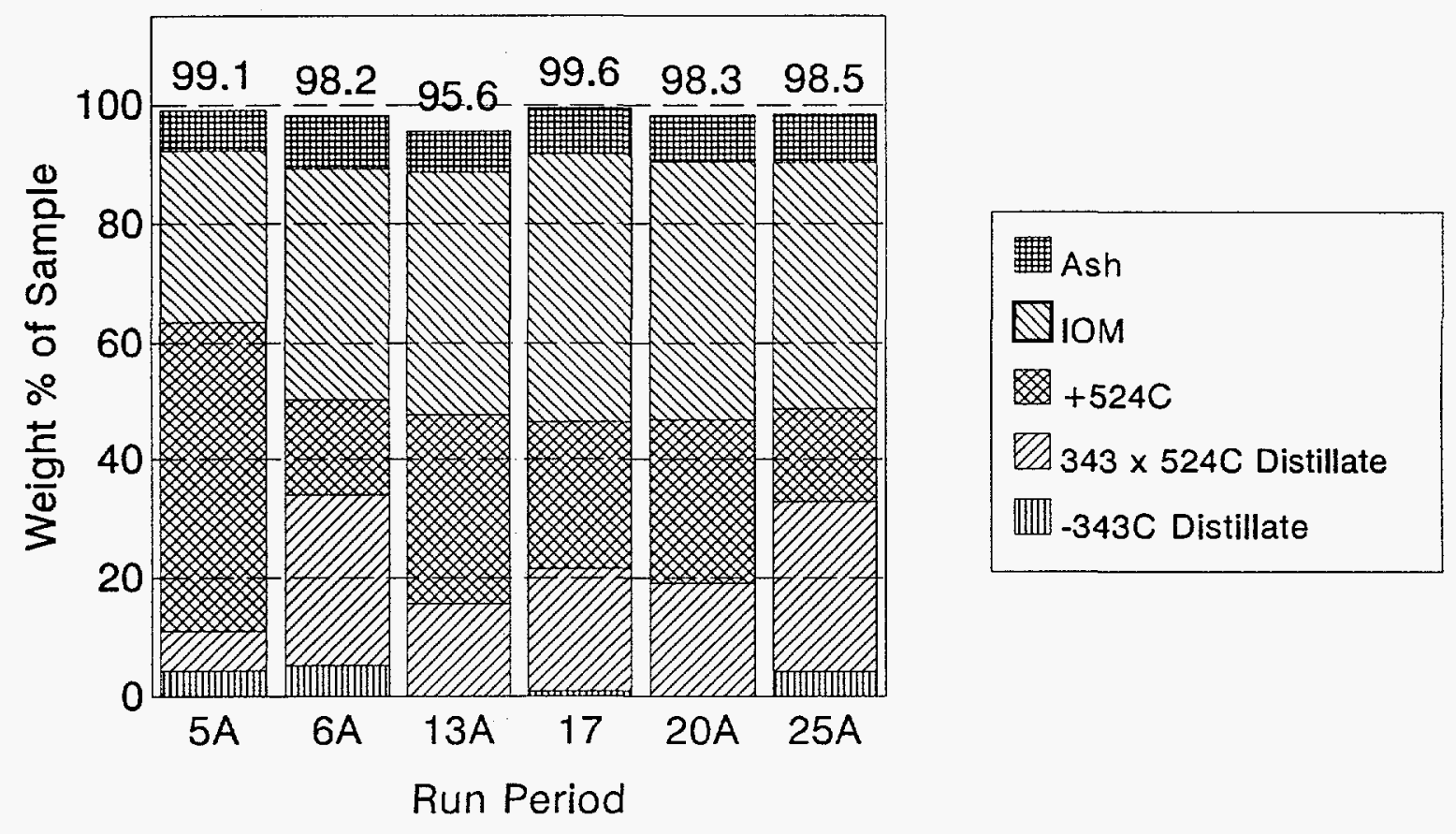

Figure 1. Component Distribution of Feed Slurry Samples from HTI Run ALC-1. 


\section{HTI Run ALC-1 \\ Component Distribution Of 0-6 Bottom Samples}

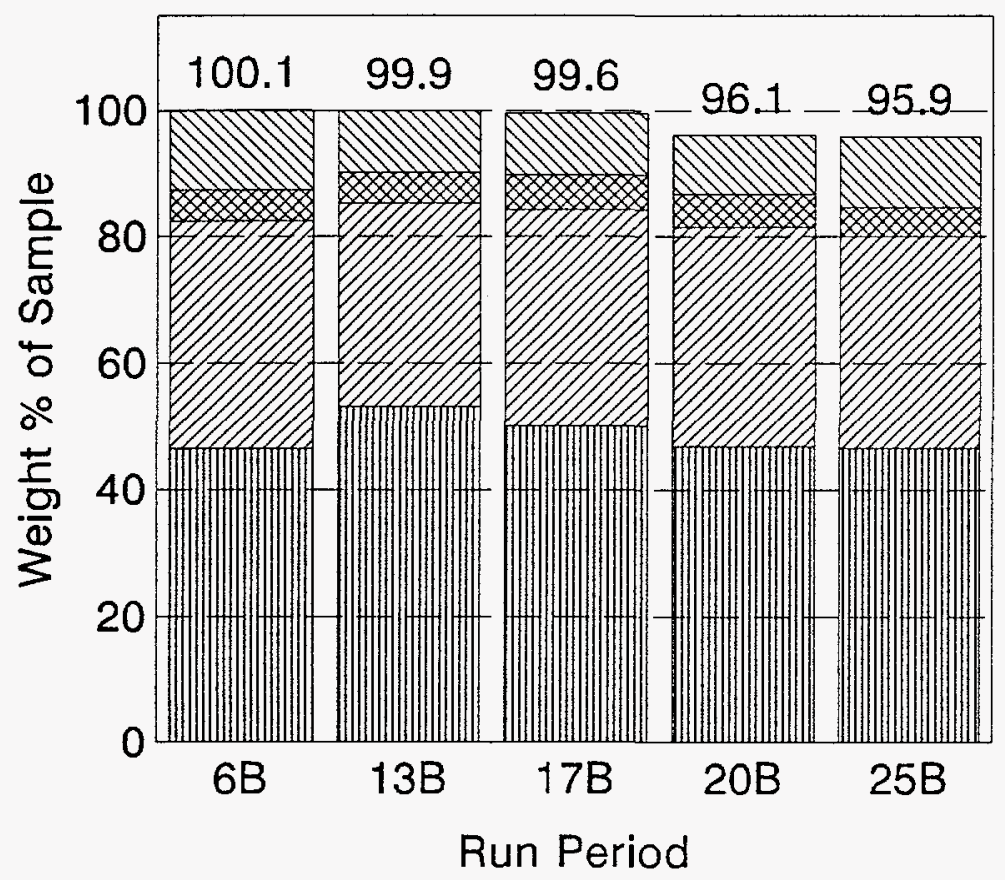

Ash

IOM

THF Sol. Resid

睞 -454 C Distillate

Run Period

Figure 2. Component Distribution of O-6 Bottoms Samples from HTI Run ALC-1. 


\section{HTI Run ALC-1 \\ Component Distribution Of PFC Samples}

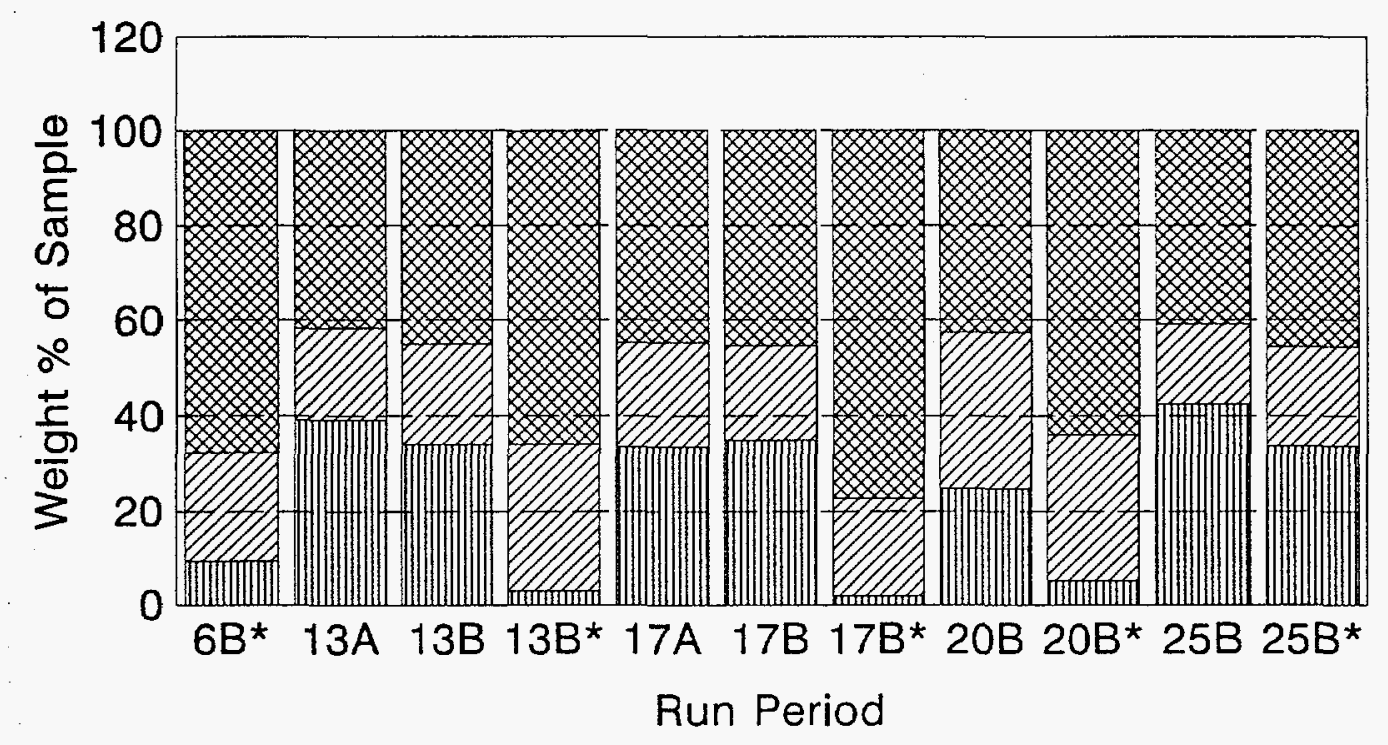

㽢 Resid(by diff.) $\mathbb{Z}$ IOM Ash

* Toluene Extracted

Figure 3. Component Distribution of Pressure-Filter Cake (PFC) Samples from HTI Run ALC-1. 


\section{HTI RUN ALC-1 $\%$ AROMATICS H - WHOLE SAMPLES}

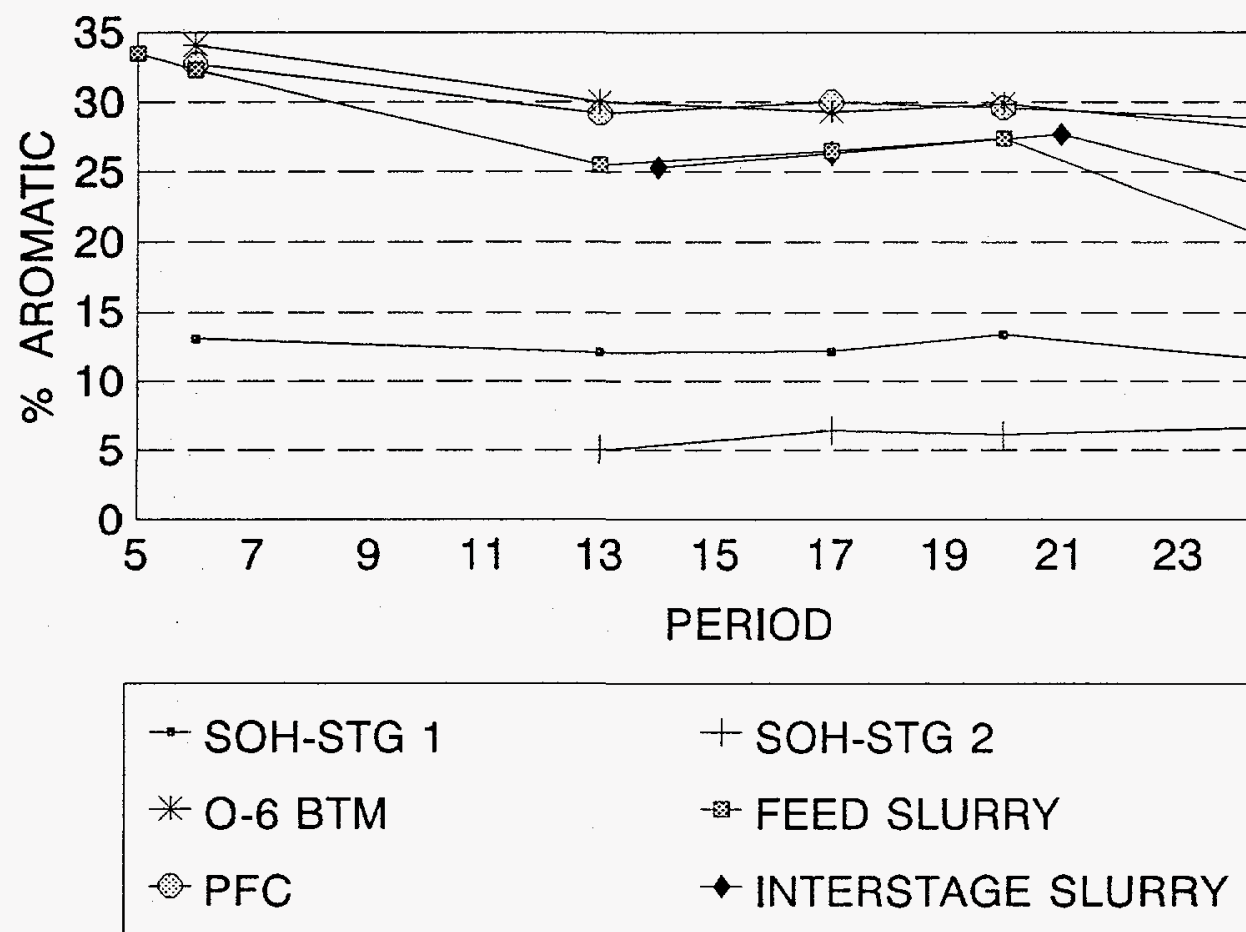

Figure 4. Proton Aromaticity of Whole Process Stream Samples from HTI Run ALC-1. 


\section{HTI RUN ALC-1 $\%$ PARAFFINIC H - WHOLE SAMPLES}

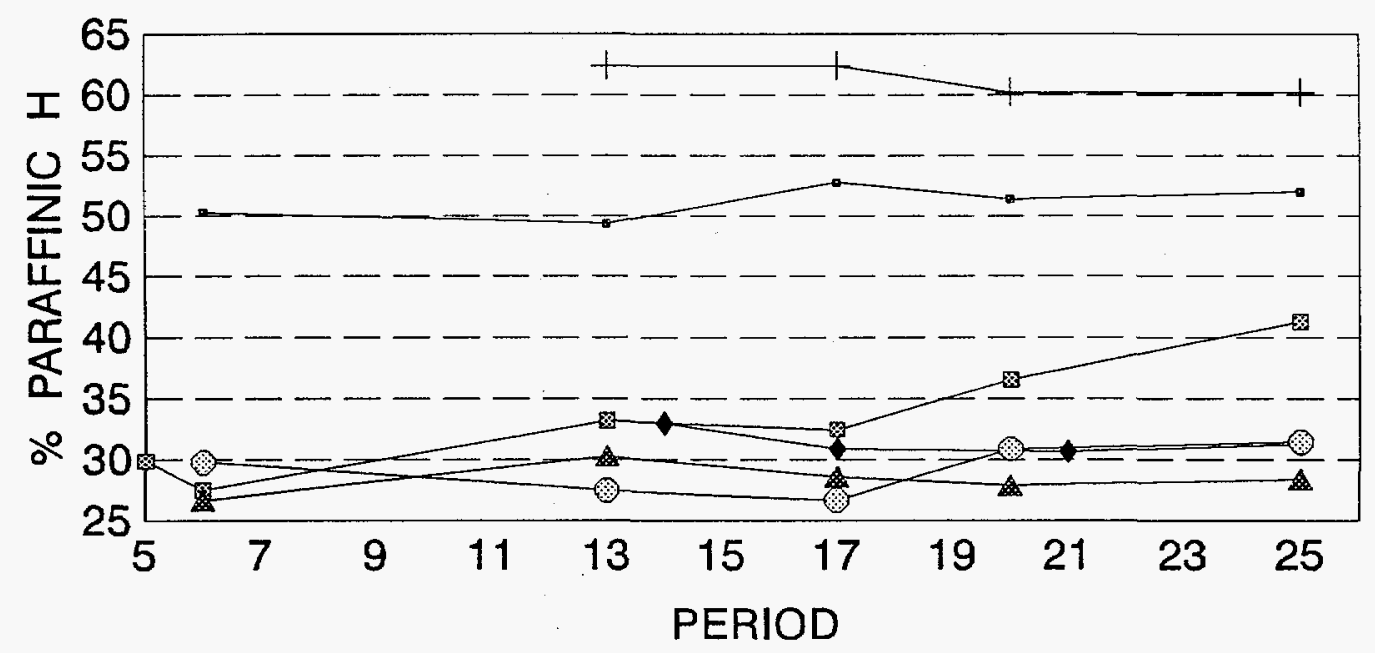

$\begin{array}{ll}- \text { SOH-STG } 1 & + \text { SOH-STG } 2 \\ \star \text { O-6 BTM } & - \text { FEED SLURRY } \\ - \text { PFC } & - \text { INTERSTAGE SLURRY }\end{array}$

Figure 5. Paraffinic Proton Content of Whole Process Stream Samples from HTI Run ALC-1. 


\section{HTI RUN ALC-1 MICROAUTOCLAVE COAL CONVERSION}

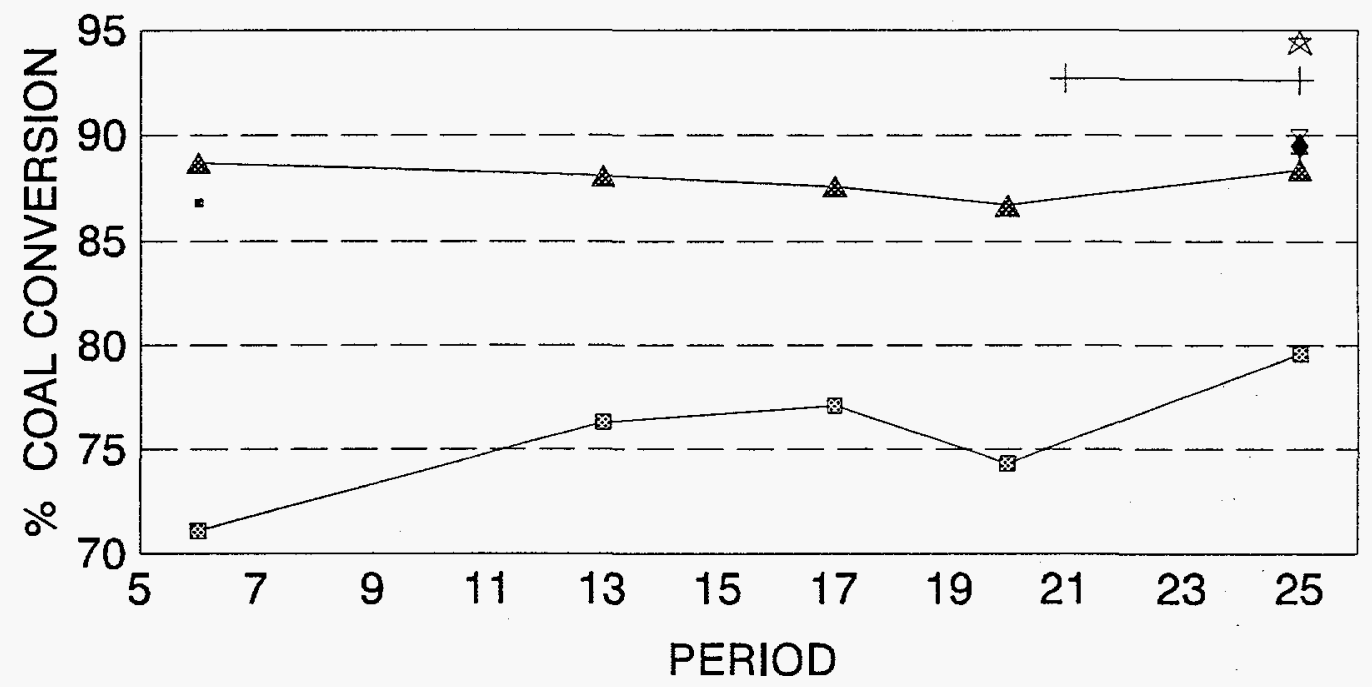

- L-814 START-UP OIL

0-6 WHOLE SAMPLE

$\star$ VSOH HYDRO. DW. $+343 \mathrm{C}$
+ VSOH-HYDRO. DEWAXED
\$-6 DISTILLATE
$\Xi V S O H$ DEWAXED
- VSOH IBPX524C

Figure 6. Donor Solvent Quality of Selected Whole and Distillate Samples from HTI Run ALC-1. 


\section{HTI Run ALC-1 \\ Phenolic $\mathrm{OH}$ Content Of Whole Samples and Resids}

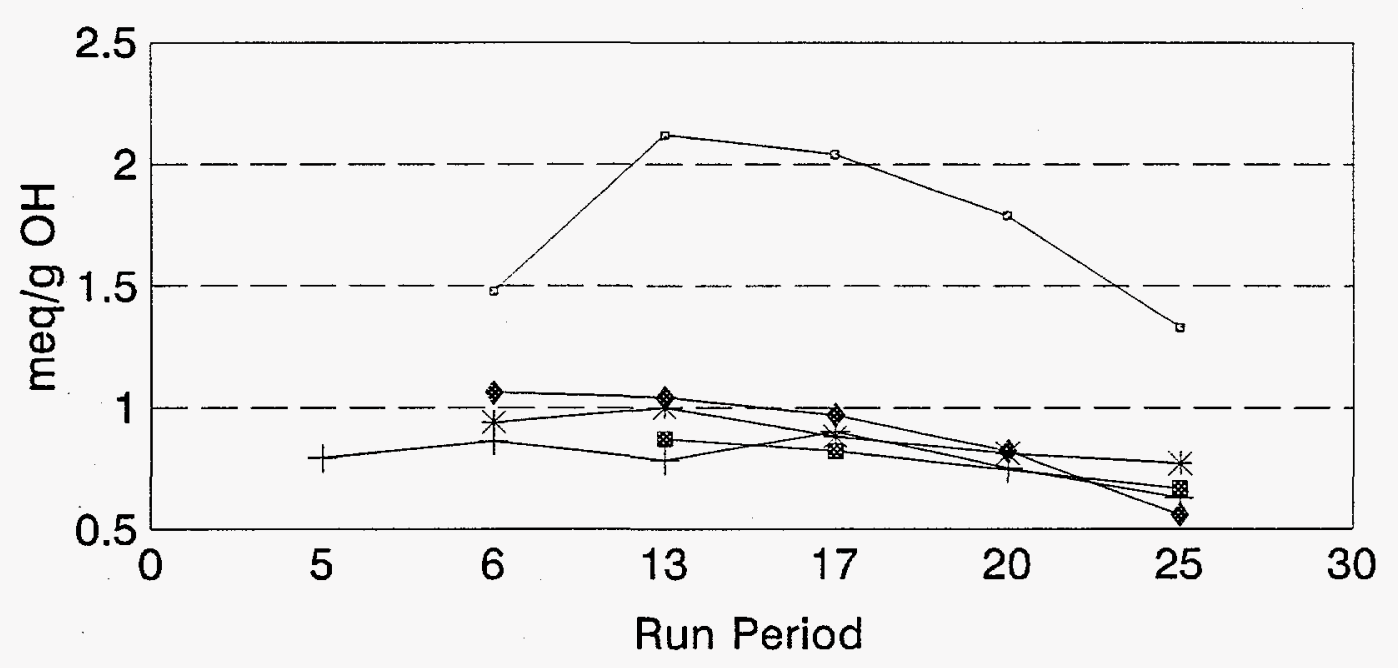

$\rightarrow$ SOH STG 1 -WHOLE + FEED SLURRY-RESID

* 0-6 BTMS-RESID - PFC-WHOLE

$-0-6$ BTMS-DIST.

Figure 7. Phenolic -OH Concentration in Process Stream Samples from HTI Run ALC-1. 


\section{APPENDIX 1}

RECALIBRATION OF FTIR SPECTROSCOPIC PHENOLIC -OH METHOD 


\section{RECALIBRATION OF FTIR SPECTROSCOPIC METHOD FOR PHENOLIC -OH DETERMINATION}

\section{INTRODUCTION}

Replacement of CONSOL's original Fourier-transform infrared (FTIR) spectroscopic system (Nicolet 170SX) with a new model (Nicolet Magna 550) required that the FTIR method used for determination of phenolic - $\mathrm{OH}$ concentration in liquefaction samples be recalibrated for the new system. Implementation of the method on the new system also required that the method be reprogrammed to facilitate analysis and calculations performed on the new system. To accomplish these tasks, several steps were performed. Solutions were prepared of six phenol standards at four concentrations each. The spectrum of each standard solution was run on the new FTIR instrument system, and the spectra were visually inspected for quality. The net absorbance of the phenolic $-\mathrm{OH}$ peak was measured using a re-implemented version of the software. The absorbance-concentration relation of each standard was checked statistically for consistency with the other concentrations of each standard. A regression was used to obtain the new calibration relationship, and the calbration was tested by analyzing authentic coal liquids that were run on both the old and new systems.

\section{METHOD DETAILS AND VALIDATION}

The six model compounds that were previously used as standards ${ }^{1-3}$ were selected as standards for recalibration, except that 2,3,5-trimethyl phenol was substituted for the 2,4,5-trimethyl phenol used in the original calibration. Four solutions of each phenol standard were prepared and run on the new FTIR instrument. The spectra were visually inspected for quality, and repeats were run as needed. The solution concentrations were randomly designed to span a range of 8.5-50.0 $\mathrm{mm}$-meq/ $\mathrm{L}$ in concentration times pathlength $(P L)$. to cover the range of the original calibration. The solution concentration of phenolic $-\mathrm{OH}$ was calculated from the spectrum of each standard solution using the original calibration equation

conc $^{*} P L$ (in $\left.\mathrm{mm}-\mathrm{meq} / \mathrm{L}\right)=53.61^{*}$ net absorbance -0.1892

( \pm 1.8 as the standard error of the $Y$ estimate),

where net absorbance is the height of the phenol O-H stretch peak (found in the $3200-3400 \mathrm{~cm}^{-1}$ region) minus the baseline absorbance (integration over the $3650-3750 \mathrm{~cm}^{-1}$ region). From this, the sample concentration is obtained by

conc (in meq/g) $=\left(\text { conc }{ }^{*} P L\right)^{*}($ solution Vol in $L) /\left((P L \text { in } m m)^{*}(\right.$ sample wt in $\left.g)\right)$

Because the sample (weight) concentration of phenolic $-\mathrm{OH}$ in each phenol standard should be independent of solution concentration, this value (meq $\mathrm{OH} / \mathrm{g}$ sample) provides a measure of agreement within the set of four solutions of each standard. These values were subjected to the Q-test for statistical outliers (at the $90 \%$ and higher confidence level). Questionable solutions of each standard were remade and rerun to obtain a new result to replace each outlying result. After a good set of data was obtained, regressions of net absorbance on conc*PL and vice versa were calculated using the four data points for each of the six standard phenols (i.e., six sets of regressions were calculated). Regressions of absorbance on conc ${ }^{*} P L$ give the extinction coefficient or absorptivity of each of the phenols as the slope, whereas regressions of conc* $\mathrm{PL}$ on absorbance give the slope and intercept in a form useful as a calibration line for analysis. The 
six individual regressions of four points each gave regression coefficients $\left(R^{2}\right)$ of 0.993 to 0.999 , whereas the overall regression of 24 points gave a regression coefficient $\left(R^{2}\right)$ of 0.955 (see Table 1). The individual regressions assured that the solution standards were of high quality. Figure 1 shows all the standard data, with identification of the subsets of different phenol standards. The tabulated data are given in Table 2.

The new calibration is given as

conc $^{*} P L($ in $m m-m e q / L)=50.20( \pm 2.32){ }^{*}$ net absorbance +1.19

( \pm 2.63 as the standard error of the $Y$ estimate), $R^{2}=0.955$

from which the sample concentration is obtained as described above. The calibration error (standard error of the $Y$ estimate) is higher than in the original calibration; this is discussed later.

In order to validate the calibration, a set of 10 samples was analyzed both on the old instrument with the old calibration, and on the new instrument with the new calibration. The sample set included several representative coal liquids, and a few model compounds (standards). For three of the samples, runs were made in duplicate on the new instrument and weighted averages were used to determine bias (i.e., each replicate contributed one-half as much to the total error as each single determination). The validation results are shown in Table 3 . Figure 2 shows a parity plot of new versus reference (original) concentrations. In general, the peak position reported by the new calibration was biased $1.8 \mathrm{~cm}^{-1}$ lower than the original data (range $1-3 \mathrm{~cm}^{-1}$ lower).

For the entire set ( 3 standards, 2 high concentration samples, 5 normal concentration samples), the concentration bias was $0.00 \mathrm{meq} / \mathrm{g}$, and the regression equation was

new conc. $=$ old conc. ${ }^{*} 1.01( \pm 0.02)-0.04( \pm 0.19$ as the standard error of the $Y$ estimate $), R^{2}=0.997$

For the set without the standards (2 high concentration samples, 5 normal concentration samples), the concentration bias was $-0.08 \mathrm{meq} / \mathrm{g}$, and the regression equation was

new conc. $=$ old conc. ${ }^{*} 0.92( \pm 0.01)+0.02( \pm 0.08$ as the standard error of the $Y$ estimate $), R^{2}=0.998$

For the set of normal concentration samples ( 5 normal concentration samples), the concentration bias was $0.02 \mathrm{meq} / \mathrm{g}$, and the regression equation was

new conc. $=$ old conc. ${ }^{*} 1.04( \pm 0.01)+0.003( \pm 0.01$ as the standard error of the $Y$ estimate $), R^{2}=0.999$

The results indicate a trivial bias of less than 0.02 meq/g in magnitude for the samples. The phenolic extract sample with a low new result created a larger magnitude bias when the standards were omitted from the validation. The discrepancy between old and new results was larger for samples with higher phenolic $-\mathrm{OH}$ concentrations (i.e. $>1.0 \mathrm{meq} / \mathrm{g}$, which is outside the most common range for coal liquids). The largest concentration differences observed between the old instrument and new instrument amounted to a relative error of ca. $7 \%$. The calibration regression showed a larger error than the original calibration, but the new one involved more standards and was much more rigorously set up from a statistical standpoint. It is likely that the earlier calibration underestimated the actual error. It appears that the calibration regression also represents a relative error of ca. $5 \%$, but this error analysis may not be rigorous. A calculation of the calibration error for each sample was implemented in the new version of the method, according to the following formula: 
conc error due to calibration (in meq/g) $=2.63^{*}($ solution Vol in $L) /\left((P L \text { in } \mathrm{mm})^{*}(\right.$ sample wt in $g)$ )

This approach essentially disregards the error in measurement of solution volume, sample weight, and cell pathlength, and assumes that the error arising from differences in the model compounds used as standards is the largest error contributor. A printout of the computer program used to process each spectrum and perform the concentration calculations and a printout of the report output are shown in Listings 1 and 2. Because the old FTIR command set was not fully implemented on the new FTIR system, software implementation of the method on the new instrument was not straightforward. This required an advanced macro language (Visual Basic 3.0) implementation, rather than the simpler standard macro language provided by Nicolet.

\section{REFERENCES}

1. Burke, F. P.; Winschel, R. A. "Recycle Slurry Oil Characterization, Technical Report, October 1983 through March 1984", DOE/PC 30027-60, March 1985.

2. Burke, F. P.; Winschel, R. A.; Robbins, G. A. "Recycle Slurry Oil Characterization, Final Report, October 1980 through March 1985", DOE/PC 30027-61, March 1985.

3. Robbins, G. A.; Winschel, R. A.; Burke, F. P. "Phenolic -OH as a Process-Performance Indicator in Two-Stage Liquefaction", Am. Chem. Soc. Div. Fuel Chem. Prepr. 1985, 30(4), 155-163. 
TABLE A1-1

\section{REGRESSION RESULTS FOR FTIR SPECTROSCOPIC PHENOLIC -OH METHOD RECALIBRATION}

\begin{tabular}{|c|c|c|c|c|c|}
\hline \multicolumn{3}{|c|}{ OverallRegression Output: $X=$ Conc. $Y=A b s$. } & \multicolumn{3}{|c|}{ Overall Regression Output: $X=A$ abs. $Y=$ Conc. } \\
\hline Constant & & 0.0027 & Constant & & 1.19 \\
\hline Std Err of Y Est & & 0.0512 & \multicolumn{2}{|l|}{ Std Err of Y Est } & 2.63 \\
\hline R Squared & & 0.9552 & R Squared & & 0.96 \\
\hline \multicolumn{2}{|c|}{ No. of Observations } & 24 & \multicolumn{2}{|c|}{ No of Observations } & 24 \\
\hline \multicolumn{2}{|c|}{ Degrees of Freedom } & 22 & \multicolumn{2}{|c|}{ Degrees of Freedom } & 22 \\
\hline$X$ Coefficient(s) & 0.019 & & $x$ Coefficient(s) & 50.2 & \\
\hline Std Err of Coef. & 0.0009 & & Std Err of Coef. & 2.319 & \\
\hline \multicolumn{3}{|c|}{$\begin{array}{l}\text { Individual Regression Output: } X=\text { Conc., } Y=A b s \text {. } \\
\text { No. of Observations }=4 \text {, Degrees of Freedom }=2\end{array}$} & \multicolumn{3}{|c|}{$\begin{array}{l}\text { Individual Regression Output: } X=A b s ., Y=C o n c \\
\text { No. of Observations }=4 \text {. Degrees of Freedom }=2\end{array}$} \\
\hline \multicolumn{6}{|c|}{ 2.3.5-trimethylphenol } \\
\hline Constant & & 0.0192 & Constant & & -1.05 \\
\hline Std Err of $Y$ Est & & 0.0111 & \multicolumn{2}{|l|}{ Std Err of $Y$ Est } & 0.62 \\
\hline R Squared & & 0.9991 & R Squared & & 0.9991 \\
\hline$x$ Coefficient(s) & 0.0177 & & $\times$ Coefficient(s) & 56.42 & \\
\hline Std Err of Coef. & 0.0004 & & Std Err of Coef. & 1.21 & \\
\hline \multicolumn{6}{|c|}{ 2.5-ditertbutylphenol } \\
\hline Constant & & -0.027 & Constant & & 1.58 \\
\hline Std Err of Y Est & & 0.0241 & \multicolumn{2}{|l|}{ Std Err of Y Est } & 1.20 \\
\hline R Squared & & 0.9929 & R Squared & & 0.9929 \\
\hline$X$ Coefficient(s) & 0.02 & & $X$ Coefficient(s) & 49.58 & \\
\hline Std Err of Coef. & 0.0012 & & Std Err of Coef. & 2.96 & \\
\hline \multicolumn{6}{|c|}{ 2-naphthol } \\
\hline Constant & & -0.022 & Constant & & 1.02 \\
\hline Std Err of Y Est & & 0.0133 & \multicolumn{2}{|l|}{ Std Err of $Y$ Est } & 0.58 \\
\hline R Squared & & 0.9981 & R Squared & & 0.9981 \\
\hline X Coefficient(s) & 0.0228 & & $x$ Coefficient(s) & 43.84 & \\
\hline Std Err of Coef. & 0.0007 & & Std Err of Coef. & 1.35 & \\
\hline \multicolumn{6}{|c|}{ 9-phenanthrol } \\
\hline Constant & & -0.002 & Constant & & 0.19 \\
\hline Std Err of $Y$ Est & & 0.0163 & \multicolumn{2}{|l|}{ Std Err of Y Est } & 0.96 \\
\hline R Squared & & 0.9969 & R Squared & & 0.9969 \\
\hline$X$ Coefficient(s) & 0.017 & & $x$ Coefficient(s) & 58.81 & \\
\hline Std Err of Coef. & 0.0007 & & Std Err of Coef. & 2.32 & \\
\hline \multicolumn{6}{|c|}{ hydroquinone } \\
\hline Constant & & -0.03 & Constant & & 1.51 \\
\hline Std Err of Y Est & & 0.0097 & \multicolumn{2}{|l|}{ Std Err of Y Est } & 0.48 \\
\hline R Squared & & 0.9993 & $R$ Squared & & 0.9993 \\
\hline$x$ Coefficient(s) & 0.0201 & & $X$ Coefficient(s) & 49.77 & \\
\hline Std Err of Coef. & 0.0004 & & Std Err of Coef. & 0.94 & \\
\hline \multicolumn{6}{|c|}{ p-cresol } \\
\hline Constant & & -0.005 & Constant & & 0.39 \\
\hline Std Err of Y Est & & 0.0198 & \multicolumn{2}{|l|}{ Std Err of $Y$ Est } & 1.00 \\
\hline R Squared & & 0.9957 & R Squared & & 0.9957 \\
\hline$X$ Coefficient(s) & 0.0196 & & $X$ Coefficient(s) & 50.75 & \\
\hline Std Err of Coef & 00009 & & Std Err of Coef & 236 & \\
\hline
\end{tabular}


TABLE A1-2

\section{STANDARDS DATA FOR FTIR SPECTROSCOPIC PHENOLIC -OH METHOD RECALIBRATION}

\begin{tabular}{|c|c|c|c|c|c|c|c|}
\hline $\begin{array}{l}\text { Std. } \\
\text { No. }\end{array}$ & Phenol Standard & $\begin{array}{c}\text { Mol } \\
\text { weight }\end{array}$ & $\begin{array}{l}\text { Eq/ } \\
\text { Mol }\end{array}$ & $\begin{array}{c}\text { g used per } \\
25 \mathrm{~mL} \\
\text { sol'n }\end{array}$ & $\begin{array}{c}\text { Actual Conc., } \\
\text { meq/L (PL=1 } \\
\mathrm{mm})\end{array}$ & $\begin{array}{l}\text { Net } \\
\text { Abs. }\end{array}$ & $\begin{array}{c}\text { Pk. Pos. } \\
\mathrm{cm}^{-1}\end{array}$ \\
\hline 7 & 2,3,5-trimethylphenol & 136.19 & 1 & 0.0299 & 8.78 & 0.170 & 3322 \\
\hline 21 & 2,3,5-trimethylphenol & 136.19 & 1 & 0.0856 & 25.14 & 0.477 & 3318 \\
\hline 8 & 2,3,5-trimethylphenol & 136.19 & 1 & 0.1202 & 35.30 & 0.637 & 3320 \\
\hline 9 & 2,3,5-trimethylphenol & 136.19 & 1 & 0.1654 & 48.58 & 0.879 & 3320 \\
\hline 12 & 2,5-ditertbutylphenol & 206.33 & 1 & 0.0932 & 18.07 & 0.317 & 3316 \\
\hline 11 & 2,5-ditertbutylphenol & 206.33 & 1 & 0.1502 & 29.12 & 0.564 & 3314 \\
\hline 22 & 2,5-ditertbutylphenol & 206.33 & 1 & 0.1654 & 32.07 & 0.639 & 3313 \\
\hline 10 & 2,5-ditertbutylphenol & 206.33 & 1 & 0.2389 & 46.31 & 0.885 & 3314 \\
\hline 23 & 2-naphthol & 144.17 & 1 & 0.0436 & 12.10 & 0.248 & 3279 \\
\hline 15 & 2-naphthol & 144.17 & 1 & 0.1000 & 27.75 & 0.624 & 3280 \\
\hline 13 & 2-naphthol & 144.17 & 1 & 0.1207 & 33.49 & 0.742 & 3280 \\
\hline 14 & 2-naphthol & 144.17 & 1 & 0.1328 & 36.85 & 0.807 & 3280 \\
\hline 24 & 9-phenanthrol & 194.23 & 1 & 0.0758 & 15.61 & 0.248 & 3245 \\
\hline 18 & 9-phenanthrol & 194.23 & 1 & 0.1060 & 21.83 & 0.384 & 3252 \\
\hline 17 & 9-phenanthrol & 194.23 & 1 & 0.1523 & 31.36 & 0.536 & 3250 \\
\hline 16 & 9-phenanthrol & 194.23 & 1 & 0.2320 & 47.78 & 0.803 & 3248 \\
\hline 2 & hydroquinone & 110.11 & 2 & 0.0241 & 17.51 & 0.312 & 3331 \\
\hline 3 & hydroquinone & 110.11 & 2 & 0.0259 & 18.82 & 0.351 & 3330 \\
\hline 1 & hydroquinone & 110.11 & 2 & 0.0354 & 25.72 & 0.495 & 3330 \\
\hline 19 & hydroquinone & 110.11 & 2 & 0.0680 & 49.41 & 0.960 & 3332 \\
\hline 5 & p-cresol & 108.14 & 1 & 0.0404 & 14.94 & 0.278 & 3310 \\
\hline 6 & p-cresol & 108.14 & 1 & 0.0881 & 32.59 & 0.657 & 3309 \\
\hline 4 & p-cresol & 108.14 & 1 & 0.0920 & 34.03 & 0.664 & 3309 \\
\hline 20 & p-cresol & 108.14 & 1 & 0.1223 & 45.24 & 0.869 & 3310 \\
\hline
\end{tabular}


TABLE A1-3

VALIDATION DATA FOR FTIR SPECTROSCOPIC PHENOLIC -OH METHOD RECALIBRATION

\begin{tabular}{|c|c|c|c|c|c|c|c|}
\hline & & \multicolumn{2}{|c|}{ NEW } & \multicolumn{2}{|c|}{ REFERENCE } & \multicolumn{2}{|c|}{ DIFFERENCE } \\
\hline SAMPLE & DESCRIPTION & $\begin{array}{c}\text { CONC., } \\
\mathrm{meg} / \mathrm{g}\end{array}$ & $\begin{array}{c}\text { PK. POS., } \\
\mathrm{cm}^{-1}\end{array}$ & $\begin{array}{c}\text { CONC., } \\
\mathrm{meg} / \mathrm{g}\end{array}$ & $\begin{array}{c}\text { PK. POS., } \\
\mathrm{cm}^{-1}\end{array}$ & $\begin{array}{c}\text { CONC., } \\
\mathrm{meq} / \mathrm{g}\end{array}$ & $\begin{array}{c}\text { PK. POS., } \\
\mathrm{cm}^{-1}\end{array}$ \\
\hline p-cresol & Std., $M W=108.1$ & 9.36 & 3309 & 9.25 & NA & 0.11 & NA \\
\hline 3,4,5-trimethylphenol & Std., $M W=136.2$ & 7.46 & 3323 & 7.34 & NA & 0.12 & NA \\
\hline 2-naphthol & Std., $M W=144.2$ & 7.27 & 3279 & 6.94 & NA & 0.33 & NA \\
\hline$\# 3351$ & High & 1.02 & 3294 & 1.25 & 3295 & -0.22 & -1 \\
\hline \#351 (Repeat) & High & 1.07 & 3294 & 1.25 & 3295 & -0.18 & -1 \\
\hline$\# 3290$ & High & 5.80 & 3309 & 6.25 & 3311 & -0.44 & -2 \\
\hline \#1981 & Normal & 0.86 & 3296 & 0.83 & 3298 & 0.03 & -2 \\
\hline \#1981 (Repeat) & Normal & 0.88 & 3296 & 0.83 & 3298 & 0.05 & -2 \\
\hline$\$ 3239$ & Normal & 0.08 & 3315 & 0.09 & 3318 & 0.00 & -3 \\
\hline \#3239 (Repeat) & Normal & 0.09 & 3315 & 0.09 & 3318 & 0.00 & -3 \\
\hline$\# 3231$ & Normal & 0.09 & 3313 & 0.09 & 3314 & 0.00 & -1 \\
\hline$\# 3352$ & Normal & 0.89 & 3296 & 0.85 & 3297 & 0.04 & -1 \\
\hline \#2051 & Normal & $0.00^{*}$ & $3357^{*}$ & $0.00^{*}$ & $3357^{\star}$ & 0.00 & NA \\
\hline \multicolumn{3}{|c|}{ Bias as Weighted Avg. - Stds, High \& Normal } & & & & 0.00 & NA \\
\hline \multicolumn{3}{|c|}{ Bias as Weighted Avg. - High \& Normal Conc. } & & & & -0.08 & -1.7 \\
\hline \multicolumn{3}{|c|}{ Bias as Weighted Avg - Normal Conc } & & & & 002 & -18 \\
\hline
\end{tabular}

${ }^{*}$ Peak found represents a non-phenolic component. The concentrations are reported here as $0.00 \mathrm{meq} / \mathrm{g}$ for the purpose of determining bias; a more accurate representation of the concentration is "none detected". 
RE-STANDARDIZATION OF FTIR PHENOL METHOD

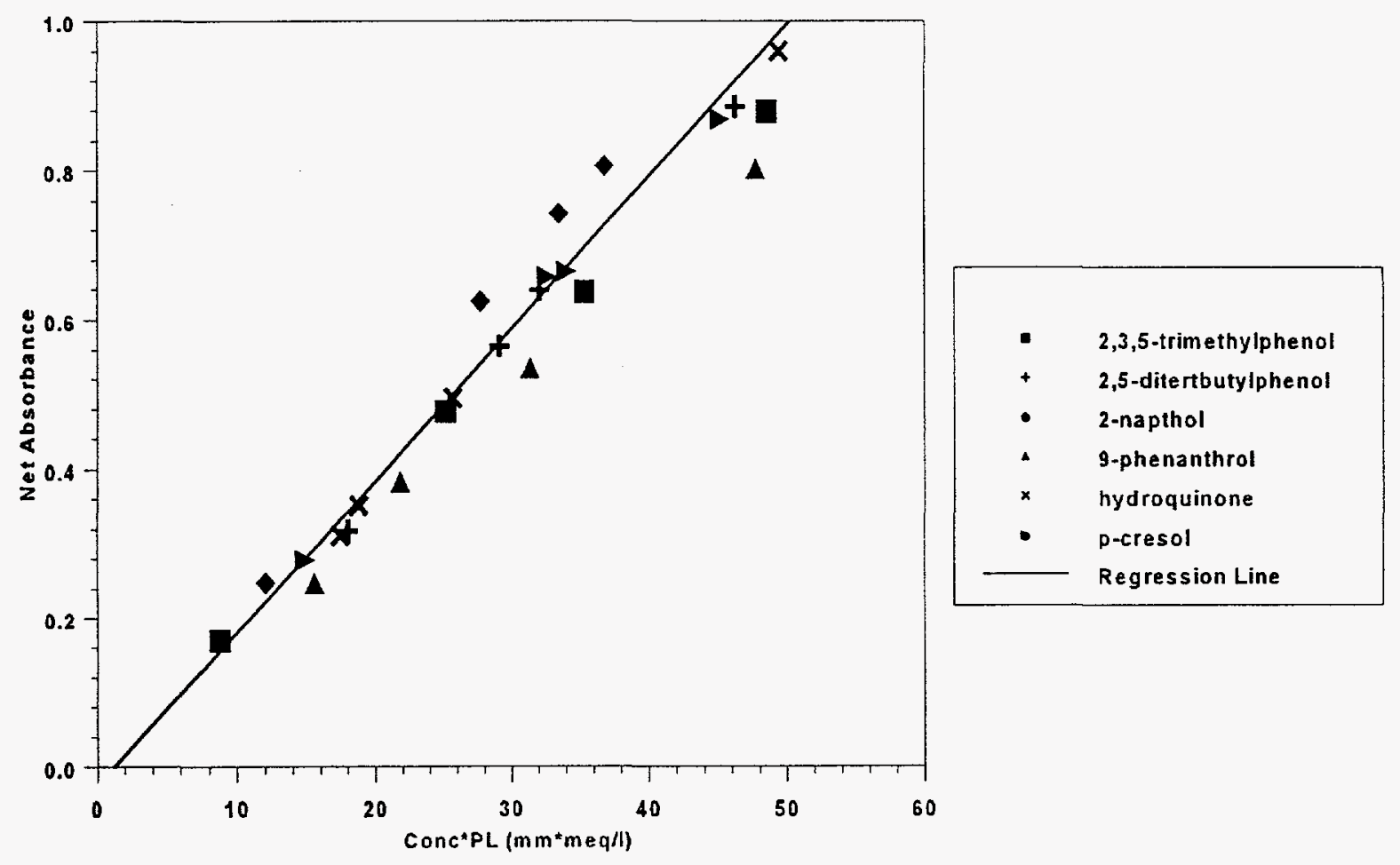

Figure A1-1. Calibration Data Showing Subsets of Each Standard and Overall Regression Line. 
PARITY PLOT FOR PHENOL METHOD VALIDATION

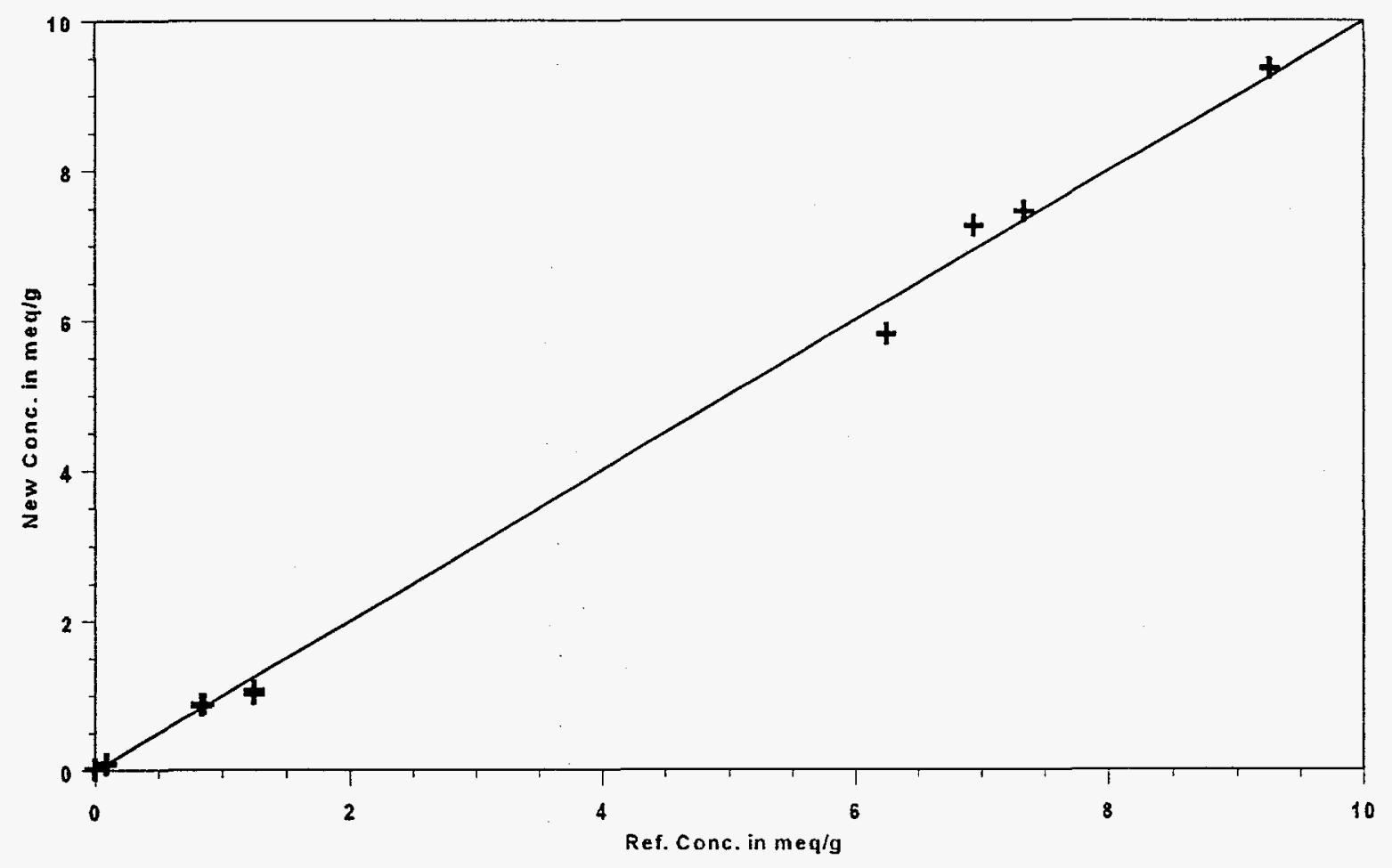

Figure A1-2. Parity Plot of Validation Results Obtained from New and Original Spectrometer and Calibration. 
Listing A1-1 - Example Report Output for FTIR Spectroscopic Phenolic -OH Determination

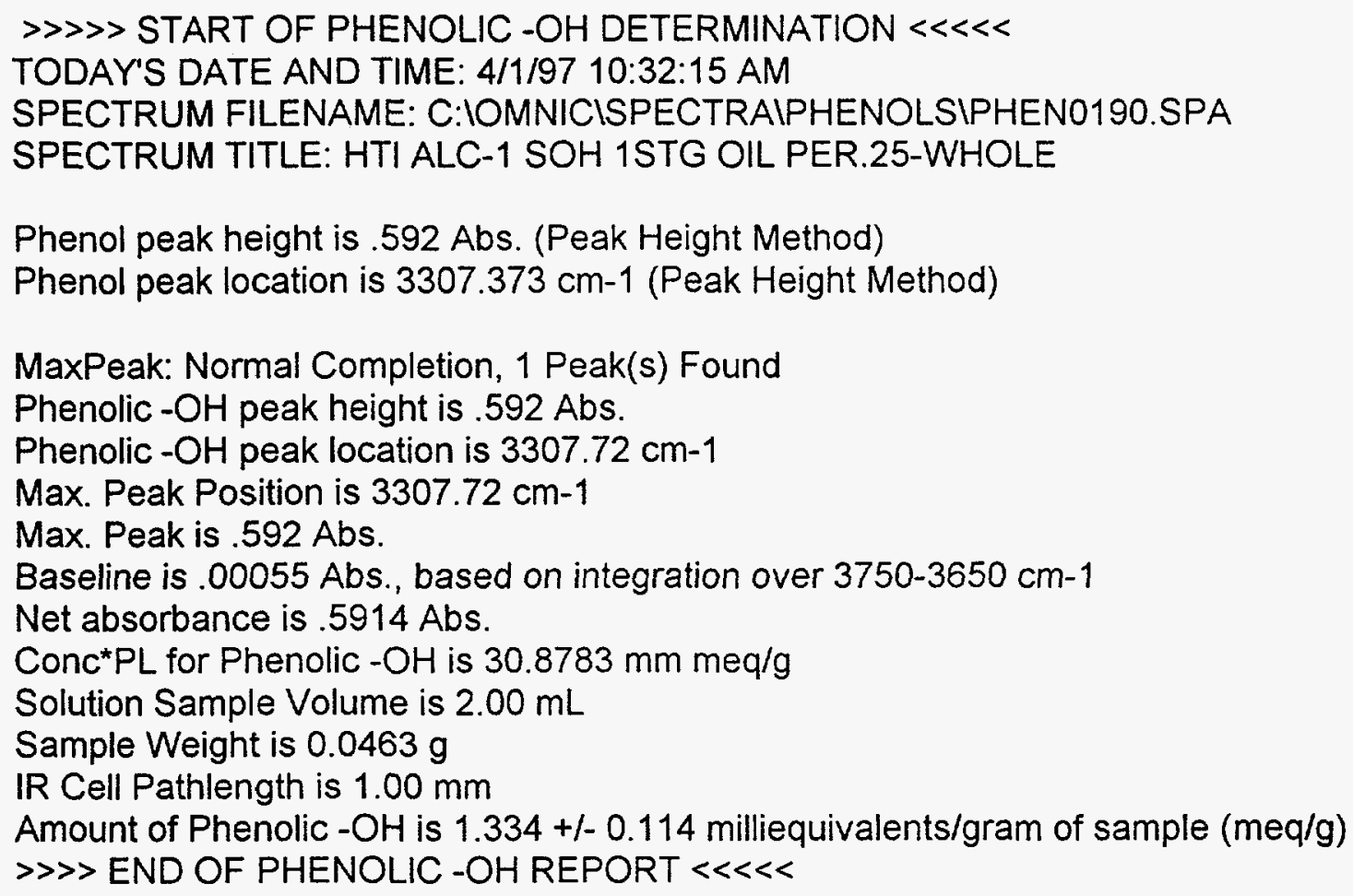


Listing A1-2 - Computer Program Listing for FTIR Spectroscopic Phenolic -OH Determination

Notes: Written in Visual Basic 3.0, Requires Nicolet Macros Pro and Nicolet OMNIC Software, Used on Nicolet Magna 550 Spectrometer

\section{PHENCLC1.FRM - 1}

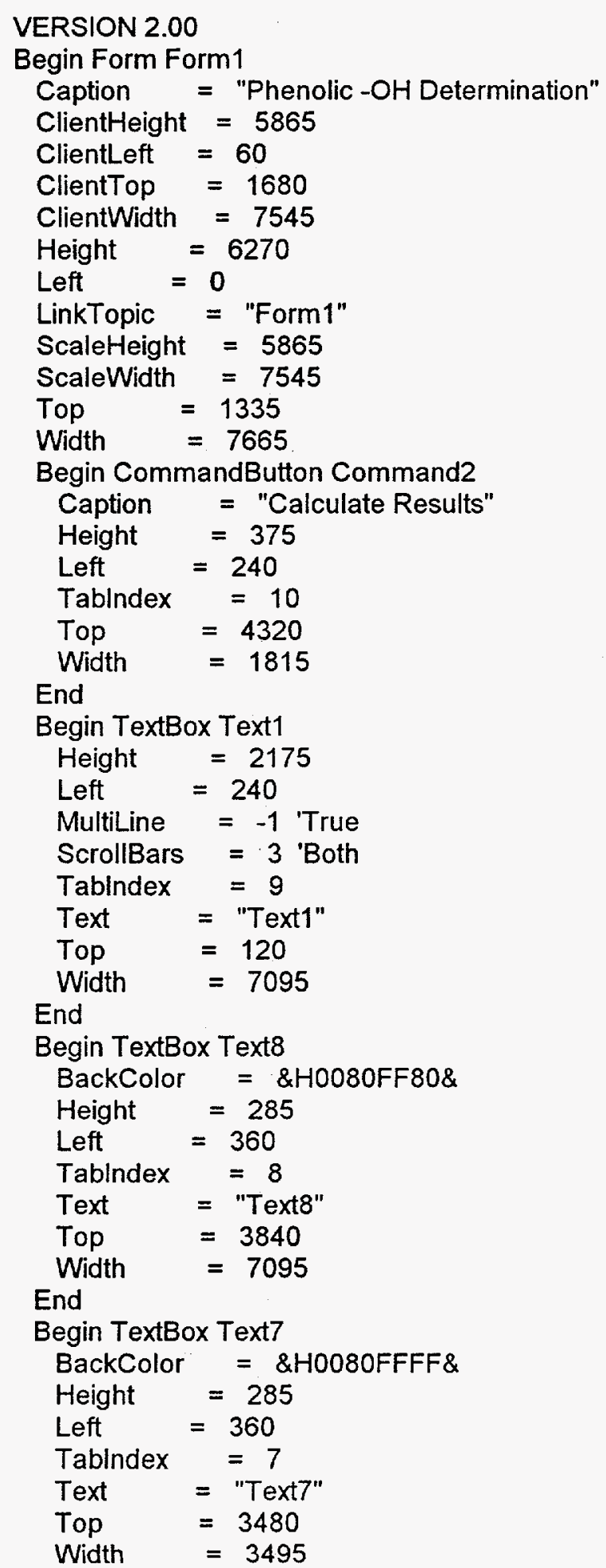




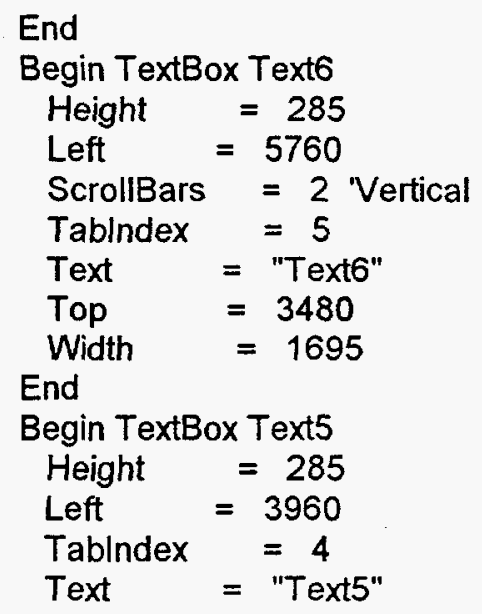

\section{PHENCLC1.FRM - 2}

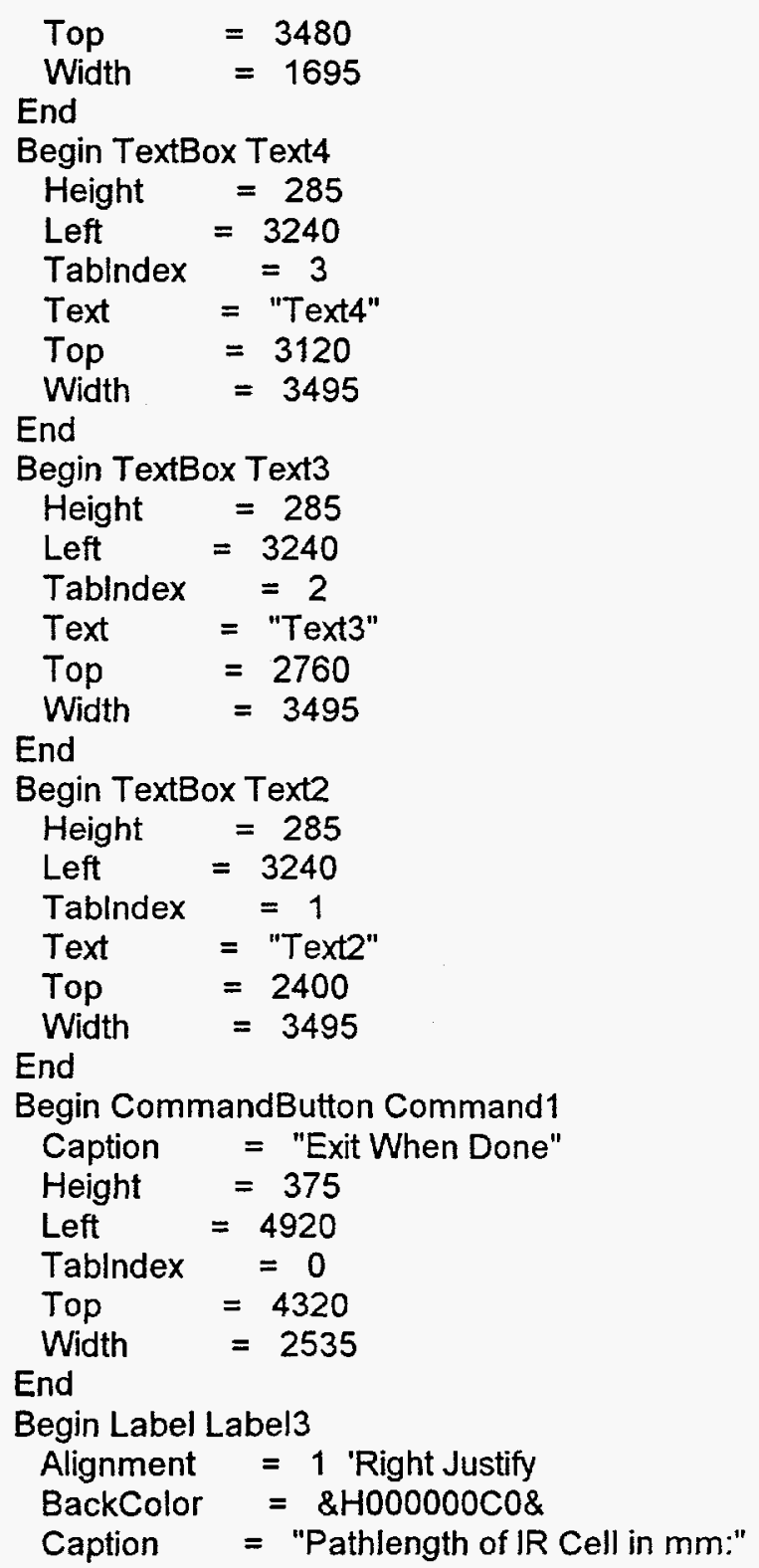




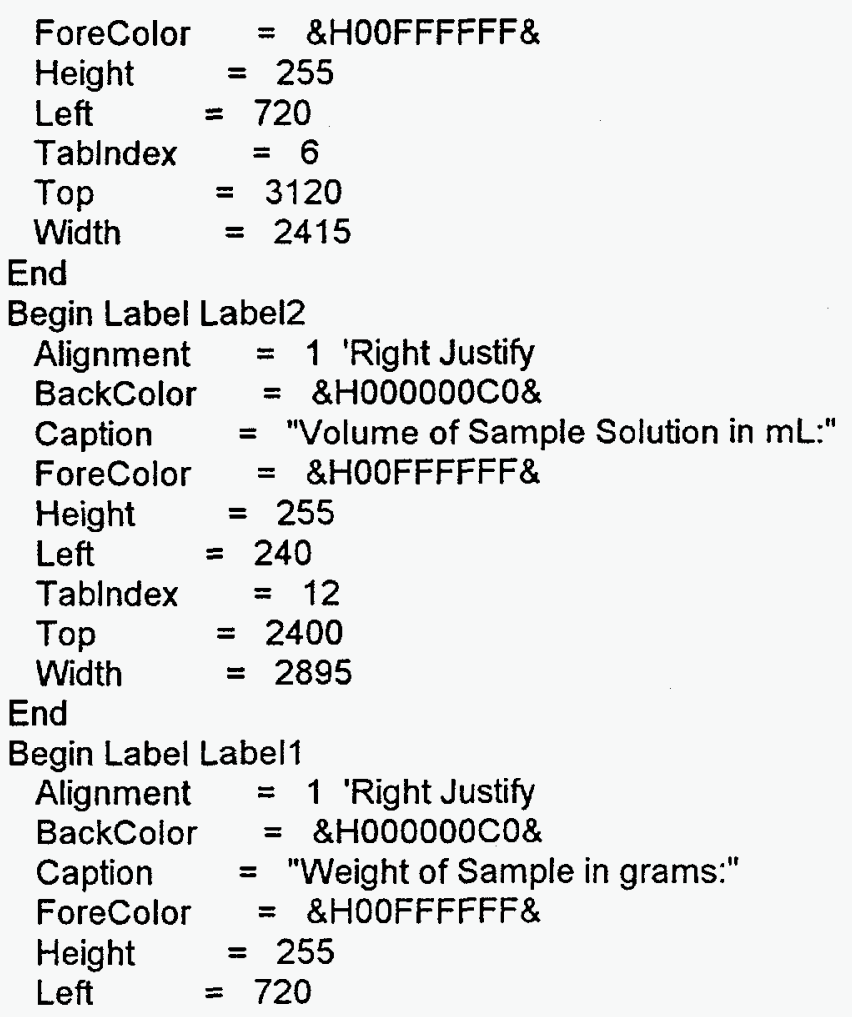

PHENCLC1.FRM - 3

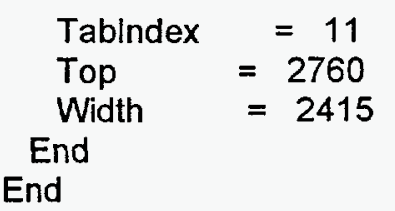

\section{PHENCLC1.FRM - 1}

Dim Results(100, 2), ResultStr, TotalLen, StartStr, EndStr, NumPeaks, MaxPeak, PeakPo s Dim NetAbs As Double, BslAbs As Double

Dim AmntPhen As Variant, ConcPL As Variant, ErrPhen As Variant Dim SpecFile, SpecTitle

Sub GetResults 0

StartStr $=1$

TotalLen = Len(ResultStr)

- Error Trap for 0 peaks

If $\ln S \operatorname{tr}(1$, ResultStr, "No peaks found") > 0 GoTo NoPeaks StartStr = InStr(StartStr, ResultStr, "Position:")

ResultStr $=$ Mid (ResultStr, StartStr)

StartStr $=10$

EndStr = InStr (StartStr, ResultStr, "Intensity:")

Results $(1,1)=$ Val(Mid(ResultStr, StartStr, EndStr - StartStr))

PeakPos $=$ Results $(1,1)$

StartStr $=$ EndStr

ResultStr $=$ Mid (ResultStr, StartStr)

StartStr $=11$

EndStr = InStr(StartStr, ResultStr, "Position:") 


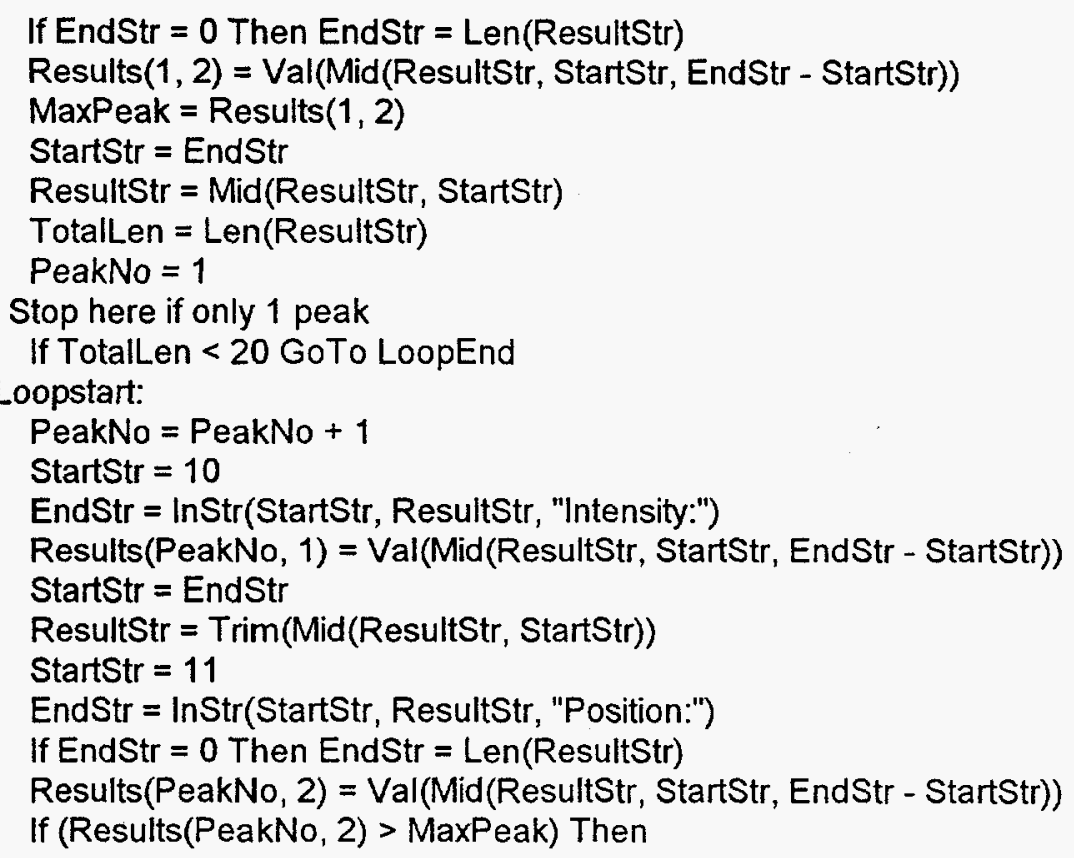

\section{PHENCLC1.FRM - 2}

MaxPeak $=$ Results $($ PeakNo, 2)

PeakPos $=$ Results $($ PeakNo, 1$)$

End If

ResultStr $=$ Mid(ResultStr, EndStr)

TotalLen $=$ Len(ResultStr)

If (TotalLen > 30) GoTo Loopstart Else GoTo LoopEnd

NoPeaks:

Text6. Text = "MaxPeak: No peaks were found"

GoTo SubEnd

LoopEnd:

Text6.Text = "MaxPeak: Normal Completion, " \& PeakNo \& " Peak(s) Found"

SubEnd:

Text1. Text $=$ Text1. Text \& $\operatorname{Chr}(13) \& \operatorname{Chr}(10) \&$ Text6. Text

Text6.Text = "Phenolic $-\mathrm{OH}$ peak height is " \& MaxPeak \& " Abs."

Text1. Text $=$ Text1.Text \& $\operatorname{Chr}(13) \& \operatorname{Chr}(10) \&$ Text6. Text

Text6.Text = "Phenolic - OH peak location is " \& PeakPos \& " cm-1"

Text1. Text $=$ Text1. Text \& $\mathrm{Chr}(13) \& \mathrm{Chr}(10)$ \& Text6. Text

ExecuteOMNIC "CorrectedPeakArea 3800375036503600 "

Result\$ = GetOMNIC("Result Current")

Text6. Text = "Max. Peak Position is " \& PeakPos \& " cm-1"

Text1. Text $=$ Text1. Text \& Chr(13) \& Chr(10) \& Text6. Text

Text6.Text = "Max. Peak is " \& MaxPeak \& " Abs."

Text1. Text $=$ Text1. Text \& $\operatorname{Chr}(13) \& \operatorname{Chr}(10) \&$ Text6. Text

BslAbs = GetVal(Result\$, "Uncorrected:") / 100\#

Text6. Text = "Baseline is " \& BsIAbs \& " Abs., based on integration over 3750-365

$0 \mathrm{~cm}-1 "$

Text1. Text $=$ Text1. Text \& $\operatorname{Chr}(13) \& \operatorname{Chr}(10) \&$ Text6. Text

NetAbs = Format(MaxPeak - BsIAbs, "\#0.00\#")

Text7. Text = "Net absorbance is " \& NetAbs \& " Abs."

Text1. Text $=$ Text1. Text \& $\operatorname{Chr}(13) \& \operatorname{Chr}(10) \&$ Text7.Text

ConcPL $=$ Format $((50.2$ *NetAbs $)+1.19, " \# 0.00 \# ")$

Text8.Text $=$ "Conc ${ }^{\star} \mathrm{PL}$ for Phenolic $-\mathrm{OH}$ is " \& ConcPL \& " mm meq $/ \mathrm{g} "$

Text1. Text $=$ Text1. Text \& $\operatorname{Chr}(13) \& \operatorname{Chr}(10) \&$ Text8. Text 
Text2. Text $=" 2.0 "$

Text3. Text $=" 1.0 "$

Text4. Text $=" 1.0 "$

End Sub

Sub Command1_Click 0

\section{PHENCLC1.FRM - 3}

" ExecuteOMNIC "Select First"

" ExecuteOMNIC "Set Display YAxisMode FullScale"

" ExecuteOMNIC "CloseWindow No"'Phenol sample"

" ErrMsgBox

End

End Sub

Sub Command2_Click 0

SampleVol = Format(Val (Text2.Text), "\#0.00")

SampleWt $=$ Format $(V a l(T e x t 3$. Text), "\#0.00\#")

PathLen = Format(Val(Text4.Text), "\#0.00")

Text6.Text = "Solution Sample Volume is " \& SampleVol \& " $\mathrm{mL}$ "

Text1.Text $=$ Text1.Text \& Chr(13) \& Chr(10) \& Text6.Text

Text6.Text = "Sample Weight is " \& SampleWt \& " $\mathrm{g}$ "

Text1.Text $=$ Text1. Text \& $\operatorname{Chr}(13) \& \operatorname{Chr}(10) \&$ Text6. Text

Text6.Text = "IR Cell Pathlength is " \& PathLen \& " mm"

Text1. Text $=$ Text1.Text \& $\operatorname{Chr}(13) \& \operatorname{Chr}(10) \&$ Text6. Text

AmntPhen $=$ ConcPL $•$ SampleVol $/($ PathLen $*$ SampleVt * 1000)

AmntPhen = Format (AmntPhen, "\#0.00\#")

ErrPhen $=2.63$ * SampleVol $/($ PathLen * SampleWt • 1000)

ErrPhen = Format (ErrPhen, "\#0.00\#")

Text8.Text $=$ "Amount of Phenolic $-\mathrm{OH}$ is " \& AmntPhen \& " $+/-$ " \& ErrPhen \& " mill iequivalents/gram of sample (meq/g)"

Text1. Text $=$ Text1.Text $\& \operatorname{Chr}(13) \& \operatorname{Chr}(10) \&$ Text8. Text

,

Text6. Text $=\operatorname{Chr}(13) \& \operatorname{Chr}(10) \&$ " $\gg \gg \gg>$ START OF PHENOLIC - OH DETERMINATION $\ll<<$

Result\$ = "TODAY'S DATE AND TIME: " \& Date \& " " \& Time

Text6. Text $=\operatorname{Chr}(13) \& \operatorname{Chr}(10) \& \operatorname{Text6}$. Text \& $\operatorname{Chr}(13) \& \operatorname{Chr}(10) \&$ Result\$

Result\$ = "SPECTRUM FILENAME: " \& SpecFile

Text6. Text $=\operatorname{Chr}(13) \& \operatorname{Chr}(10) \&$ Text6. Text \& $\operatorname{Chr}(13) \& \operatorname{Chr}(10)$ \& Result\$

Result\$ = "SPECTRUM TITLE: " \& SpecTitle

Text6. Text $=$ Text6. Text \& $\operatorname{Chr}(13) \& \operatorname{Chr}(10) \&$ Result\$ \& $\operatorname{Chr}(13) \& \operatorname{Chr}(10)$

Text1. Text $=\operatorname{Chr}(13) \& \operatorname{Chr}(10) \& \operatorname{Text6}$. Text \& $\operatorname{Chr}(13) \& \operatorname{Chr}(10) \&$ Text1. Text

Text6. Text $=$ " $\gg>$ END OF PHENOLIC -OH REPORT $\ll<<"$

Text1. Text $=$ Text1. Text \& $C h r(13) \& C h r(10) \&$ Text6. Text

Clipboard.Clear

Clipboard.SetText Text1.Text

ExecuteOMNIC "StartLogging"'C:IOMNICILOGIPHENOLS.LOG"

ExecuteOMNIC "LogDisplay On"

ExecuteOMNIC "Paste"

ErrMsgBox

ExecuteOMNIC "StopLogging"

ErrMsgBox

LogFile = "C:IOMNICILOGIPHEN96aa.txt"

TotalLen $=$ Len $($ Result\$)

Open LogFile For Append Access Read Write Shared As \#1 Len = TotalLen 
Result\$ $=$ Text1. Text

Print \#1, Result\$;

Close \#1

Text6.Text = "Last Error was " \& Err

End Sub

PHENCLC1.FRM - 4

Sub Form_Load 0

Load OmTalk

$1 \%=$ StartOMNIC $\left(1, "{ }^{\prime \prime}\right)$

ExecuteOMNIC "DisplayLimits $40004000.01 .2 "$

ExecuteOMNIC "Set Display ScaleAll True"

SpecFile = GetOMNIC ("Spectrum Filename")

SpecTitle = GetOMNIC ("Spectrum Title")

ExecuteOMNIC "Set Display Mode OverlayMode"

ExecuteOMNIC "Set Display YAxisMode FuliScale"

ExecuteOMNIC "DisplayLimits $400030000.01 .2 "$

ExecuteOMNIC "Set Display ScaleAll True"

ExecuteOMNIC "Set Display YAxisMode FullScale"

ExecuteOMNIC "CopySelectedSpectra"

ExecuteOMNIC "LogDisplay On"

ExecuteOMNIC "Paste"

ExecuteOMNIC "Smooth 25"

ExecuteOMNIC "PeakHeight 3300 Shift"

ErrMsgBox

Result $\$=$ GetOMNIC("Result Current")

PhenPeak = GetVal(Result\$, "Y:")

PhenPos = GetVal(Result\$, "X:")

Text1.Text = "Phenol peak height is " \& PhenPeak \& " Abs. (Peak Height Method)"

Text1. Text $=$ Text1. Text \& $\operatorname{Chr}(13) \& \operatorname{Chr}(10) \&$ "Phenol peak location is " \& PhenPo

$\mathrm{s} \&$ " cm-1 (Peak Height Method)"

Text1.Text $=$ Text1.Text \& $\operatorname{Chr}(13) \& \operatorname{Chr}(10)$

ExecuteOMNIC "Set Display YAxisMode FullScale"

ExecuteOMNIC "DisplayLimits $340032000.01 .2 "$

ExecuteOMNIC "Set Display YAxisMode FullScale"

ExecuteOMNIC "MinMax"

Result\$ = GetOMNIC("Result Current")

YMin = GetVal(Result\$, "Min:")

YMax = GetVal(Result\$, "Max:")

ExecuteOMNIC "CustomScale YMin YMax"

ExecuteOMNIC "Display"

ExecuteOMNIC "PeakPick $0.0100 "$

ErrMsgBox

ResultStr = GetOMNIC("Result Current")

Text2. Text $=$ "'"

Text3. Text $=" \cdots$

Text6. Text $=$ "'"

GetResults

End Sub 


\section{APPENDIX 2}

SAMPLE REQUEST FOR HTI RUN PB-05 
CONSOL Ine.

Research \& Development 4000 Brownsville Road Library, PA $15129-9566$ $412-854-6600$

FAX: 412-854-6613

412-854-6748

Dr. V. Pradhan

Hydrocarbon Technologies, Inc.

P. O. Box 6047

New York and Puritan Avenues

Lawrenceville, NJ 08648

Dear Vivek:

Our sample request for Run PB-05 is described below. We are requesting material in sufficient quantities to allow for sample distribution to other research groups, as needed. We understand that you cannot always provide the requested amounts, and we can work with smaller quantities.

We would like to receive from each run condition: 1) $250 \mathrm{~g}$ of separator overhead (SOH) oil; 2) $250 \mathrm{~g}$ of atmospheric still overhead (ASOH); 3) $350 \mathrm{~g}$ of continuous atmospheric still (CAS) bottoms or its equivalent, such as $0-6$ bottoms; 4) $350 \mathrm{~g}$ of pressure-filter liquid (PFL); 5) $350 \mathrm{~g}$ of pressure-filter cake (PFC); 6) $350 \mathrm{~g}$ of feed slurry; and 7) $350 \mathrm{~g}$ of interstage sample (first-stage product). Please also include: 8) $250 \mathrm{~g}$ of the start-up/make-up oil from the beginning of the run; 9) $300 \mathrm{~g}$ sample of the feed coal; 10) $300 \mathrm{~g}$ sample of the feed resid; 11) $2 \mathrm{~kg}$ sample of the unprocessed feed auto-fluff; 12) $1 \mathrm{~kg}$ sample of the simulated MSW plastics; and 13) $250 \mathrm{~g}$ of SOH oil from any operating periods in which the in-line hydrotreater is bypassed.

For these samples, we prefer aliquots of the same samples HTI is using for material balance workups (i.e., from the last period of a run condition).

Let us know of any problem areas with this request. Thank you for your assistance.

Sincerely,

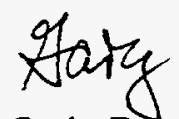

G. A. Robbins

Sr. Research Chemist

IIs

cc: $\quad$ R. M. Statnick

R. A. Winschel

S. D. Brandes

A. G. Comolli - HTI

M. A. Nowak - PETC

E. B. Klunder - PETC 


\title{
APPENDIX 3
}

\section{CRUDE OIL ASSAYS OF NET PRODUCTS OF HTI RUN PB-03}

\author{
(Periods 6,7 , and $8=$ Condition 2 ) \\ (Periods 9, 10, and $11=$ Condition 3 )
}


CONSOL Inc.

Research \& Development

4000 Brownsille Road

Library, PA 15129-9566

$412-854-6600$

FA. $412-854-6613$

$412-854-6683$

Dr. M. A. Nowak

U.S. Department of Energy

Pittsburgh Energy Technology Center

P.O. Box.10940

Pittsburgh, PA 15236

Subject: DOE Contract DE-AC22-94PC93054

Dear Mike:

Enclosed are the reports on the crude oil assays of the two products of HTI Run PB-03. The online hydrotreater was in use during Periods 6,7 , and 8 (sample HTI PB-03-6, -7, and -8), and it was by-passed during periods 9,10 , and 11 (sample HTI PB-03-9, -10, and -11). CONSOL's preparation of the samples is described in the August 1996 status report for the subject contract.* These reports will be included in one of our Technical Progress Reports.

Sincerely,<smiles>CCCCC1CCCCC1</smiles>

*and in the main budy of this report.

R. A. Winschel

Research Group Leader

Exploratory Research Group

/ls

cc: A. G. Comolli-HTI

P.-Z. Zhou - BRSC 
Consol, Inc.

$11 / 04 / 96$

4000 Brownsville Road

Library, PA 15129-9566

Attention: R.A. Winschell

Reference: Crude Assay on "HTI PB-03-6, 7, 8" received in September 1996

Mr. Winschell,

The following results are based on a composite of (2) separate distillations performed on this sample. The Distillation Data Report provides a breakdown of the separate distillations. Should you have any questions concerning this report, you may contact me at (713) $844-3311$, or by fax at (713) 844-3330.

Sincerely,

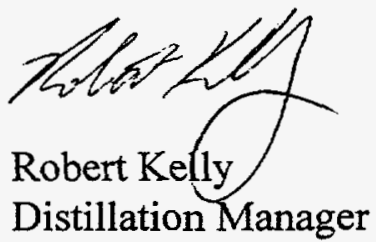




\section{Inchcape Testing Services}

\section{Caleb Brett}

November 12, 1996

Houston, Texas

Our Reference : HO/96-005162

Your Reference : PO\# 01-001-033668

Consol, Inc.

Research \& Development

4000 Brownsville Road

Library, PA 15129-9566

ATTN: R.A. Winschel

Reference: To perform "CRUDE ASSAY" on "HT1 PB-03-6,7,8" received in September 1996.

Dear Mr. Winschel:

Please find enclosed the original report on the above referenced submitted sample(s) and our invoice for services rendered.

Should you have any questions regarding this report, please do not hesitate to contact us at your convenience.

We trust you find all in order and thank you for requesting our services.

Very Truly Yours,

INCHCAPE TESTING SERVICES

CALEB BRETT U.S.A.

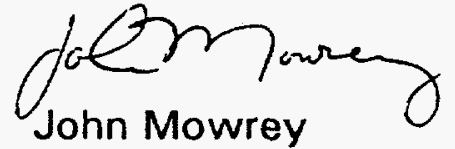

Laboratory Manager

Enclosures:

$\mathrm{JM} / \mathrm{rm}$ 


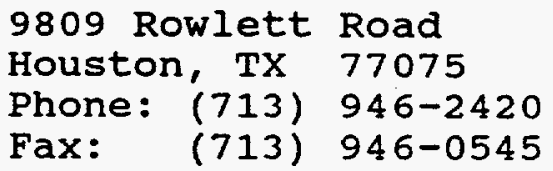

Your Ref: PO\# 01-001-033668

\section{Laboratory Report No. 96-005162-0-HOUS; 1}

Consol, Inc.

4000 Brownsville Road

Library, PA 15129-9566

For the Attention of R. A. Winschel

SAMPLE DETAILS:

SOURCE

DESCRIPTION :
8 Sample(s) received on 16-SEP-1996

Consol, Inc.

IAB REF

HTI PB-03-6, 7, 8 CRUDE OIL

Sample As Received

IBP-70 Deg. F

70-180 Deg. F

180-350 Deg. $F$

350-400 Deg. F

400-550 Deg. F

550-650 Deg. F

$650+\mathrm{Deg}$. F

$001-00$

$002-00$

003-00

004-00

005-00

006-00

007-00

008-00
CONTAINERS

RESULTS
5 Gallon Can

SEE ATTACHED SHEETS
SEALS : NONE

(TOTAL NUMBER OF PAGES 8)

Approved by:

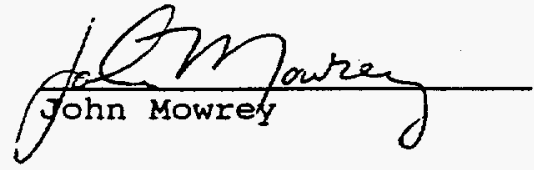


Laboratory Report No. 96-005162-0-Hous; 1 - Page 2 of 8

Sample ID

96-005162-0-HOUs-001-00

Test

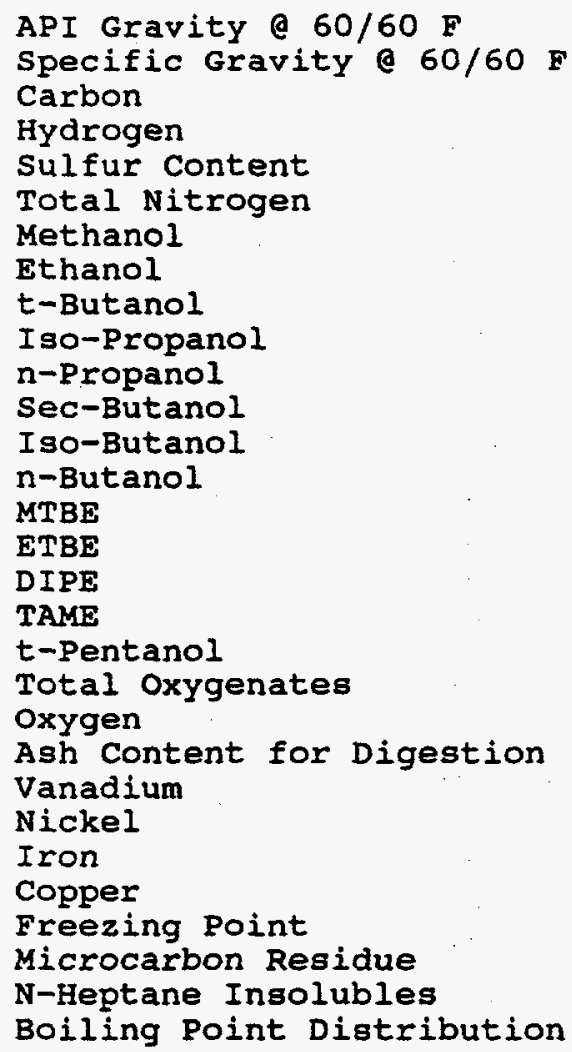

\section{Description}

HTI PB-03-6, 7, 8 CRUDE OIL

Sample As Received

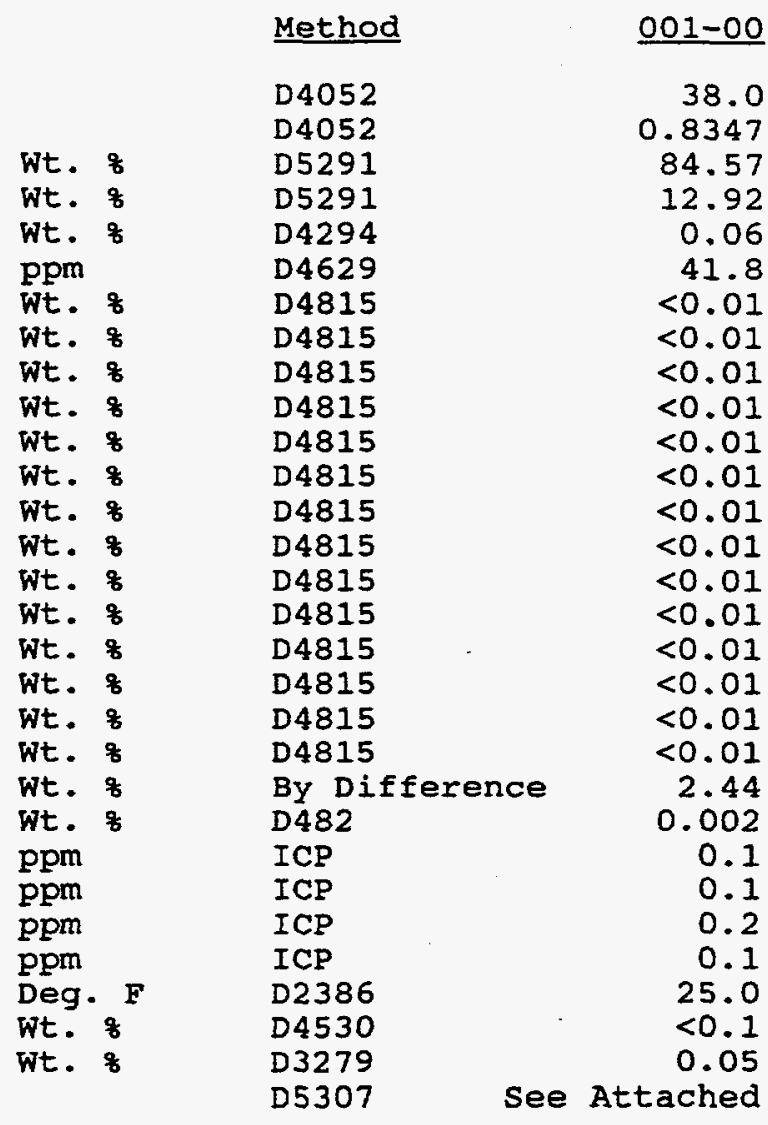

Sample ID

96-005162-0-HOUS-002-00

\section{Description}

HTI PB-03-6, 7, 8 CRUDE OIL

IBP-70 Deg. F

\section{Test}

Method

G.C.

API Gravity a $60 / 60 \mathrm{~F}$ (Charge \#1)

API Gravity a $60 / 60 \mathrm{~F}$ (Charge \#2)

Specific Gravity a $60 / 60 \mathrm{~F}$ (Charge \#1)

specific Gravity a $60 / 60$ F (Charge $\# 2$ )
G.C.

G.C.

G.C.

GC-DHA $\underline{002-00}$

113.3

109.2

0.5780

0.5880

See Attached DHA 
Inchcape Testing Services

Caleb Brett

Laboratory Report No. 96-005162-0-Hous; 1 - Page 3 of 8

Sample ID

96-005162-0-HOUS-003-00

Test

API Gravity e 60/60 F

Specific Gravity e $60 / 60$ F

Carbon

Hydrogen

Sulfur content

Total Nitrogen

Vapor Pressure

Parafins

olefins

Naphthenes

Aromatics

Total N\& A

Benzene Content

Total Acid Number

Corrosion 3 hrs $122 \mathrm{~F}$

Existent Gum

Oxidation stability

Research Octane Number

Motor Octane Number

Initial Boiling Point

a 58 Evaporated

e 108 Evaporated

e 208 Evaporated

e 308 Evaporated

a 408 Evaporated

a 508 Evaporated

(a 60\% Evaporated

e 70\% Evaporated

e 80\% Evaporated

e 908 Evaporated

e 958 Evaporated

Final Boiling Point

Recovery

Residue

Loss

\section{Description}

HTI PB-03-6, 7, 8 CRUDE OIL

70-180 Deg. F

\begin{tabular}{|c|c|c|}
\hline & Method & $003-00$ \\
\hline & D4052 & 65.7 \\
\hline & D4052 & 0.7175 \\
\hline Wt. 8 & D5291 & 84.48 \\
\hline Wt. \& & D5291 & 15.51 \\
\hline Wt. 8 & D4294 & $<0.01$ \\
\hline ppm & D4629 & 35.5 \\
\hline psi & D323 & 8.4 \\
\hline Vol. is & G.C. & 42.98 \\
\hline Vol. \& & G.C. & 0.33 \\
\hline Vol. \& & G.C. & 52.54 \\
\hline Vol. of & G.C. & 1.15 \\
\hline Vol. \& & G.C. & 53.69 \\
\hline Vol. 8 & G.C. & 1.03 \\
\hline $\mathrm{mgKOH} / \mathrm{g}$ & D974 & 0.13 \\
\hline & D130 & $3 \mathrm{~b}$ \\
\hline $\mathrm{mg} / 100 \mathrm{~mL}$ & D381 & 1 \\
\hline min. & D525 & $>240^{\circ}$ \\
\hline & D2699 & 76.1 \\
\hline & D2700 & 73.6 \\
\hline Deg. F & D86 & 107 \\
\hline Deg. F & & 132 \\
\hline Deg. F & & 137 \\
\hline Deg. F & & 143 \\
\hline Deg. F & & 148 \\
\hline Deg. F & & 153 \\
\hline Deg. F & & 157 \\
\hline Deg. F & & 161 \\
\hline Deg. F & & 165 \\
\hline Deg. F & & 168 \\
\hline Deg. F & & 172 \\
\hline Deg. F & & 174 \\
\hline Deg. F & & 181 \\
\hline Vol. \& & & 99.0 \\
\hline Vol. 8 & & 0.6 \\
\hline vol. 8 & & 0.4 \\
\hline
\end{tabular}


Sample ID

96-005162-0-HOUS-004-00

Test

API Gravity e 60/60 F

Specific Gravity a $60 / 60 \mathrm{~F}$

Carbon

Hydrogen

Sulfur content

Total Nitrogen

Mercaptan Sulfur Content

Vapor Pressure

Paraffins

olefins

Naphthenes

Aromatics

Total N\&A

Total Acid Number

Corrosion 3 hrs e 122 F

Existent Gum

Oxidation stability

Research Octane Number

Motor Octane Number

Initial Boiling point

e $5 \%$ Evaporated

(a 10\% Evaporated

e 208 Evaporated

a 308 Evaporated

a 40\% Evaporated

a 508 Evaporated

e 60z Evaporated

e 70\% Evaporated

a 80\% Evaporated

e 908 Evaporated

e 95\% Evaporated

Final Boiling Point

Recovery

Residue

Loss
Description

HTI PB-03-6, 7, 8 CRUDE OIL

180-350 Deg. F

Method

$\underline{004-00}$

D4052

D4052

Wt. \&

D5291

D5291

D4294

D4629

UOP 163

D323

G.C.

G.C.

G.C.

G.C.

G.C.

D974

D130

mgKOH/g

$\begin{array}{ll}\mathrm{mg} / 100 \mathrm{~mL} & \mathrm{D} 381 \\ \mathrm{~min} & \mathrm{D} 525\end{array}$

min. $\quad$ D2525

D2700

Deg. F

Deg. F

D86

Deg. F

Deg. F

Deg. F

Deg. F

Deg. F

Deg. F

Deg. F

Deg. F

Deg. F

Deg. F

Deg. F

Vol. \&

Vol. :

vol. 8
49.8

0.7804

85.92

14.44

0.03

22.5

25

1.4

18.56

$<0.01$

74.10

7.34

81.44

0.14

$4 \mathrm{a}$

$>240$

60.2

58.0

217

226

230

236

242

250

260

273

288

304

323

336

348

97.3

1.0

1.7 


\title{
Inchcape Testing Services
} Caleb Brett

\author{
Iaboratory Report No. 96-005162-0-Hous; 1 - Page 5 of 8
}

Sample ID

96-005162-0-HOUS-005-00

Test

API Gravity e 60/60 F

Specific Gravity a 60/60 F

Carbon

Hydrogen

Sulfur content

Total Nitrogen

Mercaptan Sulfur Content

Viscosity a $-20 \mathrm{C}$

Viscosity a $100 \mathrm{C}$

Freezing Point

Pour Point

Flash Point, TCC

Vapor Pressure

Luminometer Number

Smoke Point

Paraffins

Olefins

Naphthenes

Aromatics

Total N \& A

Naphthalenes

Total Acid Number

Corrosion $3 \mathrm{hrs}$ e $122 \mathrm{~F}$

Existent Gum

Tube Rating

Pressure Drop

Oxidation stability

Research Octane Number

Motor Octane Number

Cetane Number (Apparent)

Initial Boiling point

a 5\% Evaporated

(a) Evaporated

e 208 Evaporated

e 308 Evaporated

e 408 Evaporated

a 50\% Evaporated

a 608 Evaporated

c $70 \%$ Evaporated

e 808 Evaporated

(a) 908 Evaporated

(a) 95\% Evaporated

Final Boiling point

Recovery

Residue

Loss

Net Heat of Combustion

Description

HTI PB-03-6, 7, 8 CRUDE OIL

350-400 Deg. F

Method

D4052

D4052

Wt. \&

Wt. o

Wt. \&

ppm

ppm

cst

cst

Deg. F

Deg. $\mathbf{F}$

Deg. F

psi

mm

Vol. \&

Vol. \&

Vol. \&

Vol. :

Vol. \&

Vol. \%

$\mathrm{mgKOH} / \mathrm{g}$

$\mathrm{mg} / 100 \mathrm{~mL}$

$\mathrm{mmHg}$

min.

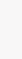

Deg. F

Deg. F

Deg. F

Deg. F

Deg. F

Deg. F

Deg. F

Deg. F

Deg. F

Deg. F

Deg. F

Deg. F

Vol. 8

Vol. \&

Vol. \&

BTU/1b

D56

D32 3

D1740

D1322

G.C.

G.C.

G.C.

G.C.

G.C.

D1840

D974

D130

D381

D3241

D525

D2699

D2 700

D613

D86
005-00

36.2

0.8437

86.38

13.17

0.03

55.8

16

3.832

0.569

$-76.0$

$<-94.0$

135

0.2

D3241

42

18

13.13

2.40

60.58

23.89

84.47

0.36

0.08

$4 a$

4.0

$>125.0$

$>240$

54.7

52.1

29.9

356

360

361

361

363

365

366

368

371

374

378

382

401

99.0

1.0

0.0

18318 
Laboratory Report No. 96-005162-0-HOUS; 1 - Page 6 of 8

Sample ID

96-005162-0-Hous-006-00

Test

API Gravity @ 60/60 F Specific Gravity a $60 / 60 \mathrm{~F}$ Carbon

Hydrogen

Sulfur Content

Total Nitrogen

Basic Nitrogen

Mercaptan Sulfur Content

Viscosity e $-20 \mathrm{C}$

Viscosity e $40 \mathrm{C}$

Viscosity a $100 \mathrm{C}$

Freezing point

Pour Point

Aniline Point

Flash Point, TCC

Luminometer Number

Smoke Point

Parafins

Olefins

Naphthenes

Aromatics

Total N\&A

Naphthalenes

Corrosion 3 hrs a 122 F

Existent Gum

Tube Rating

Pressure Drop

Cetane Number

Initial Boiling point

a 58 Evaporated

a 10\% Evaporated

a 20\% Evaporated

a 30\% Evaporated

a 408 Evaporated

a 50\% Evaporated

a 608 Evaporated

e 708 Evaporated

a $80 \%$ Evaporated

(a) 908 Evaporated

a 95\% Evaporated

Final Boiling point

Recovery

Residue

Loss

Net Heat of Combustion

Total Acid Number
Description

HTI PB-03-6, 7, 8 CRUDE OIL

400-550 Deg. F

Method

006-00

D4052

D4052

Wt. \&

D5291

Wt. \& D5291

Wt. \& D4294

ppm D4629

Ppm UOP269

ppm UOP163

CSt D445

CSt D445

cSt D445

Deg. F D2386

Deg. $F \quad$ D 97

Deg. F D611

Deg. $F$ D56

D1740

$\mathrm{mm}$

D1322

Vol. \&

G.C.

G.C.

Vol. \& G.C.

Vol. \& G.C.

Vol. \& G.C.

vol. \&

D1840

D130

$\mathrm{mg} / 100 \mathrm{~mL} \quad$ D381

D3241

mmHg

D613

Deg. $F \quad$ D86

27.5

0.8898

87.79

12.14

0.02

31.8

22

17

12.65

2.203

0.952

$-43.0$

$-49.0$

91.0

195

23

11

11.52

13.50

39.10

35.88

74.98

5.11

Deg. F

Deg. F

Deg. F

Deg. F

Deg. F

Deg. F

Deg. F

Deg. F

Deg. F

Deg. F

Deg. F

Deg. F

vol. \&

Vol. 8

Vol. \&

BTU/1b

$\mathrm{mgKOH} / \mathrm{g}$
D1405

D974
$>4.0$

$>125.0$

31.6

428

436

442

446

451

457

464

471

480

491

503

508

524

99.0

0.9

0.1

17937

0.03 


\section{Inchcape Testing Services}

Caleb Brett

Laboratory Report No. 96-005162-0-Hous; 1 - Page 7 of 8

Sample ID

96-005162-0-HOUS-007-00

Test

API Gravity e $60 / 60$ F

Specific Gravity @ $60 / 60 \mathrm{~F}$

Carbon

Hydrogen

sulfur Content

Total Nitrogen

Basic Nitrogen

Viscosity e $40 \mathrm{C}$

Viscosity e $100 \mathrm{C}$

Pour Point

Aniline Point

Flash Point (Method A)

Paraffins

Olefins

Naphthenes

Aromatics

Total $N \& A$

Bromine Number

Corrosion 3 hrs a 122 F

Cetane Number

simulated Distillation
Description

HTI PB-03-6, 7, 8 CRUDE OIL

550-650 Deg. F

\begin{tabular}{|c|c|}
\hline Method & $007-00$ \\
\hline D4052 & 20.9 \\
\hline D4052 & 0.9285 \\
\hline D5291 & 88.65 \\
\hline D5291 & 12.19 \\
\hline D4294 & 0.02 \\
\hline D4629 & 19.0 \\
\hline UOP2 69 & 9 \\
\hline D445 & 7.080 \\
\hline D445 & 1.938 \\
\hline D97 & 10.4 \\
\hline D611 & 109.0 \\
\hline D93 & $>200$ \\
\hline G.C. & 20.89 \\
\hline G.C. & 23.10 \\
\hline G.C. & 8.26 \\
\hline G.C. & 47.75 \\
\hline G.C. & 56.01 \\
\hline D1159 & 0.2 \\
\hline $\begin{array}{l}\text { D130 } \\
\text { D613 }\end{array}$ & $\begin{array}{r}1 \mathrm{a} \\
34.8\end{array}$ \\
\hline
\end{tabular}

Wt. \&

Wt. 8

Wt. \&

ppm

ppm

cst

cst

Deg. F

Deg. F

Deg. F

Vol. \&

Vol. is

Vol. 8

Vol. \&

Vol. \&

D2887
20.9

12.19

0.02

9.0
9

.080

10.4

109.0

3.10

8.26

6.01

0.2

34.8

See Attached 
Caleb Brett

Laboratory Report No. 96-005162-0-Hous; 1 - Page 8 of 8

Sample ID

96-005162-0-HOUS-008-00

Test

API Gravity e $60 / 60 \mathrm{~F}$ Specific Gravity e $60 / 60 \mathrm{~F}$ Carbon

Hydrogen

sulfur content

Total Nitrogen

Basic Nitrogen

Viscosity e $40 \mathrm{C}$

Viscosity e $100 \mathrm{C}$

Pour Point

Aniline Point

Flash Point (Method A)

Microcarbon Residue

Corrosion $3 \mathrm{hrg}$ e $122 \mathrm{~F}$

Cetane Number

Initial Boiling point

a 58 Recovery

a 108 Recovery

a 208 Recovery

a 308 Recovery

a 408 Recovery

a 508 Recovery

a $60 \%$ Recovery

a 708 Recovery

a 808 Recovery

a 908 Recovery

a 958 Recovery

Final Boiling point

Recovery

Residue + Loss
Description

HTI PB-03-6, 7, 8 CRUDE OIL

$650+$ Deg. F

\begin{tabular}{|c|c|c|}
\hline & Method & $008-00$ \\
\hline & $\begin{array}{l}\text { D } 4052 \\
\text { D4052 }\end{array}$ & $\begin{array}{r}21.4 \\
0.9254\end{array}$ \\
\hline Wt. \& & D5291 & 87.69 \\
\hline Wt. 8 & D5291 & 12.29 \\
\hline Wt. 8 & D4294 & 0.61 \\
\hline ppm & D4629 & 79.0 \\
\hline $\mathrm{ppm}$ & UOP2 69 & 34 \\
\hline cSt & $\mathrm{D} 445$ & 20.31 \\
\hline cst & D445 & 3.791 \\
\hline Deg. & D97 & 84.0 \\
\hline Deg. & D611 & 169.0 \\
\hline Deg. & D93 & $>200$ \\
\hline Wt. 8 & D4530 & $<0.1$ \\
\hline & D130 & $1 \mathrm{~b}$ \\
\hline & D613 & N/A \\
\hline Deg. & D1160 & 655 \\
\hline Deg. & & 675 \\
\hline Deg. & & 678 \\
\hline Deg. & & 683 \\
\hline Deg. & & 684 \\
\hline Deg. & & 690 \\
\hline Deg. & & 697 \\
\hline Deg. & & 708 \\
\hline Deg. & & 724 \\
\hline Deg. & & 740 \\
\hline Deg. & & 761 \\
\hline Deg. & & 779 \\
\hline Deg. & & 839 \\
\hline vol. & & 99.0 \\
\hline Vol. & & 1.0 \\
\hline
\end{tabular}




\section{Boiling Point Distribution ASTM D-2887}

\begin{tabular}{|c|c|c|c|c|c|c|c|c|}
\hline$\%$ Off & $\mathrm{BP}(\mathrm{F})$ & $\mathrm{BP}(\mathrm{C})$ & $\%$ Off & $\mathrm{BP}(\mathrm{F})$ & $\mathrm{BP}(\mathrm{C})$ & $\%$ Off & $\underline{B P(F)}$ & $\mathrm{BP}(\mathrm{C}$ \\
\hline IBP & 108.1 & 42.3 & 40 & 358.1 & 181.2 & 80 & 559.0 & $292 . \varepsilon$ \\
\hline 1 & 118.3 & 47.9 & 41 & 361.6 & 183.1 & 81 & 564.5 & $29 s$ \\
\hline 2 & 147.1 & 63.9 & 42 & 368.8 & 187.1 & 82 & 569.5 & 298 \\
\hline 3 & 159.7 & 70.9 & 43 & 374.7 & 190.4 & 83 & 575.3 & 30 \\
\hline 4 & 168.0 & 75.6 & 44 & 379.6 & 193.1 & 84 & 579.2 & 30 \\
\hline 5 & 177.4 & 80.8 & 45 & 384.6 & 195.9 & 85 & 584.5 & 30 \\
\hline 6 & 184.8 & 84.9 & 46 & 389.6 & 198.7 & 86 & 590.8 & 310 \\
\hline 7 & 189.3 & 87.4 & 47 & 393.8 & 201.0 & 87 & 597.4 & 3 \\
\hline 8 & 194.6 & 90.3 & 48 & 396.0 & 202.2 & 88 & 602.5 & 6 \\
\hline 9 & 202.1 & 94.5 & 49 & 401.4 & 205.2 & 89 & 609.3 & \\
\hline 10 & 211.2 & 99.6 & 50 & 408.2 & 209.0 & 90 & 617.0 & \\
\hline 11 & 216.1 & 102.3 & 51 & 414.0 & 212.2 & 91 & 624.9 & \\
\hline 12 & 217.8 & 103.2 & 52 & 418.5 & 214.7 & 92 & 631.9 & \\
\hline 13 & 219.4 & 104.1 & 53 & 423.5 & 217.5 & 93 & 641.6 & \\
\hline 14 & 221.4 & 105.2 & 54 & 430.3 & 221.3 & 94 & 650.5 & \\
\hline 15 & 226.4 & 108.0 & 55 & 433.3 & 222.9 & 95 & 664.5 & \\
\hline 16 & 240.7 & 115.9 & 56 & 439.1 & 226.2 & 96 & 678.1 & \\
\hline 17 & 245.5 & 118.6 & 57 & 444.7 & 229.3 & 97 & 696.1 & \\
\hline 18 & 252.7 & 122.6 & 58 & 450.2 & 232.3 & 98 & 721.9 & \\
\hline 19 & 260.0 & 126.7 & 59 & 454.4 & 234.7 & 99 & 756.6 & \\
\hline 20 & 262.8 & 128.2 & 60 & 458.5 & 236.9 & FBP & 789.7 & \\
\hline 21 & 269.7 & 132.1 & 61 & 464.0 & 240.0 & & & \\
\hline 22 & 271.0 & 132.8 & 62 & 468.4 & 242.4 & & & \\
\hline 23 & 272.0 & 133.3 & 63 & 474.4 & 245.8 & & & \\
\hline 24 & 276.1 & 135.6 & 64 & 480.0 & 248.9 & & & \\
\hline 25 & 284.5 & 140.3 & 65 & 484.2 & 251.2 & & & \\
\hline 26 & 292.6 & 144.8 & 66 & 488.2 & 253.4 & & & \\
\hline 27 & 296.7 & 147.1 & 67 & 492.4 & 255.8 & & & \\
\hline 28 & 304.6 & 151.4 & 68 & 497.9 & 258.8 & & & \\
\hline 29 & 308.3 & 153.5 & 69 & 502.4 & 261.3 & & & \\
\hline 30 & 313.0 & 156.1 & 70 & 507.7 & 264.3 & & & \\
\hline 31 & 315.8 & 157.7 & 71 & 511.7 & 266.5 & & & \\
\hline 32 & 321.6 & 160.9 & 72 & 517.5 & 269.7 & & & \\
\hline 33 & 329.9 & 165.5 & 73 & 521.4 & 271.9 & & & \\
\hline 34 & 332.7 & 167.1 & 74 & 526.0 & 274.4 & & & \\
\hline 35 & 335.7 & 168.7 & 75 & 531.8 & 277.7 & & & \\
\hline 36 & 341.0 & 171.7 & 76 & 537.1 & 280.6 & & & \\
\hline 37 & 345.8 & 174.3 & 77 & 542.2 & 283.4 & & & \\
\hline 38 & 350.6 & 177.0 & 78 & 548.4 & 286.9 & & & \\
\hline 39 & 354.3 & 179.1 & 79 & 553.1 & 289.5 & & & \\
\hline
\end{tabular}

Start Time: 0.2 minutes End Time: 24.3 minutes Area: 139559232.0

Slice Width: $0.80 \mathrm{sec}$
Sample Offset: 21859.9

Baseline Offset: 22729.7

Calibration File: 1014rt

Calibration Date: 10/16/96

Baseline Subtracted: c:ltc4lgc1011014b 


\section{Boiling Point Distribution}

\section{ASTM D-2887}

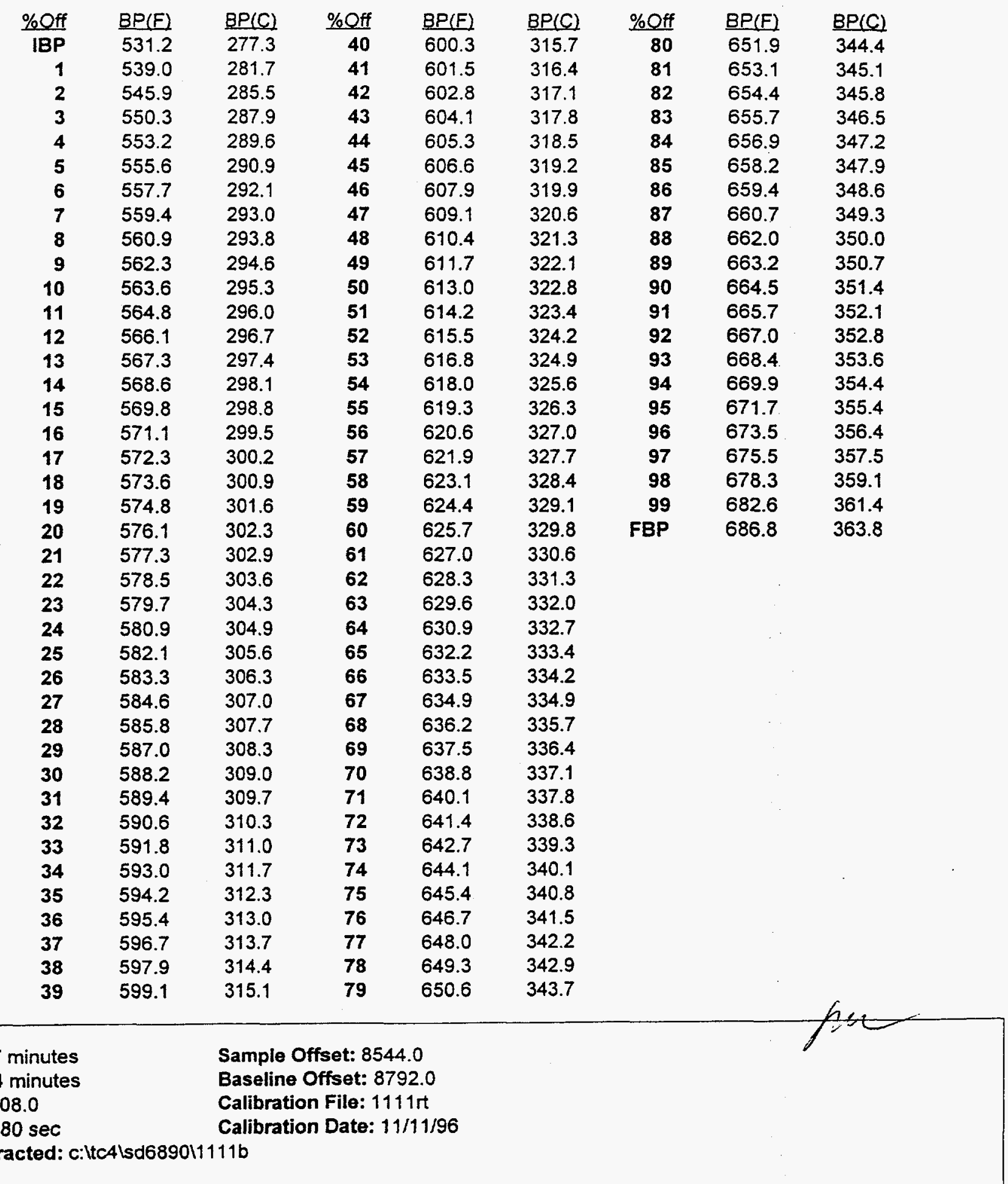


Inchcape Testing Services

Caleb Brett

WinAssay '95

Version 1.00

Final Reports

Client Name: $\quad$ Consol Inc.

Sample ID:

HTI PB-03-6,7,8 (Charge \#1)

Laboratory ID: $\quad \underline{96-005162}$

Date:

$\underline{9 / 25 / 96}$

Operator: $\quad$ Robert Kelly 


\begin{tabular}{|c|c|c|c|c|c|c|c|c|c|c|}
\hline \multicolumn{11}{|c|}{ Distillation Summary Report } \\
\hline \multicolumn{2}{|c|}{$\begin{array}{l}\text { Prepared For: } \\
\text { Sample ID: } \\
\text { Date: }\end{array}$} & \multicolumn{9}{|c|}{$\begin{array}{l}\text { Consol Inc. } \\
\text { HTI PB-03-6,7,8 (Charge \#1) } \\
9 / 25 / 96\end{array}$} \\
\hline \begin{tabular}{r|} 
Cut Temp \\
To \\
\end{tabular} & $\begin{array}{l}\text { Degrees F } \\
\end{array}$ & $\begin{array}{l}\text { DUMP } \\
\text { WT(g) } \\
\end{array}$ & $\begin{array}{l}\text { Specific } \\
\text { Gravity } \\
\end{array}$ & MLS & $\begin{array}{c}\text { LIQ } \\
\text { VOL\% } \\
\end{array}$ & $\begin{array}{c}\text { CUM. LIQ } \\
\text { VOL\% } \\
\end{array}$ & WT\% & $\begin{array}{l}\text { CUM } \\
\text { Wr\% } \\
\end{array}$ & \begin{tabular}{c|} 
API \\
GRAVITY \\
\end{tabular} & $\begin{array}{c}\text { IID } \\
\text { LIQ VOL } \% \\
\end{array}$ \\
\hline \multicolumn{11}{|c|}{ ASTM D2892 Distillation Yields } \\
\hline IBP & 70 & 61.80 & 0.5780 & 106.92 & 0.94 & 0.94 & 0.65 & 0.65 & 113.31 & 0.47 \\
\hline 70 & 180 & 896.90 & 0.7166 & 1251.60 & 11.00 & 11.94 & 9.45 & 10.10 & 65.96 & 6.44 \\
\hline 180 & 350 & 3092.80 & 0.7803 & 3963.60 & 34.85 & 46.79 & 32.58 & 42.67 & 49.84 & 29.37 \\
\hline 350 & 400 & 829.60 & 0.8430 & 984.10 & 8.65 & 55.44 & 8.74 & 51.41 & 36.35 & 51.12 \\
\hline 400 & 550 & 2613.00 & 0.8892 & 2938.60 & 25.84 & 81.28 & 27.52 & 78.94 & 27.63 & 68.36 \\
\hline 550 & 650 & 1399.90 & 0.9282 & 1508.19 & 13.26 & 94.54 & 14.75 & 93.68 & 20.95 & 87.91 \\
\hline $650+$ & & 600.00 & 0.9248 & 648.79 & 5.70 & 100.24 & 6.32 & 100.00 & 21.51 & \\
\hline
\end{tabular}

Loss (Grams): 9.8 (0.10 Wt.\%)

Distribution: (2/3) $6.5 \mathrm{~g}$ to IBP-70 F

(1/3) $3.3 \mathrm{~g}$ to $70-180 \mathrm{~F}$ 
WinAssay '95 Quality Control Applications

Cum. Mid Vol\% v. API Gravity

HTI PB-03-6,7,8 (Charge \#1)

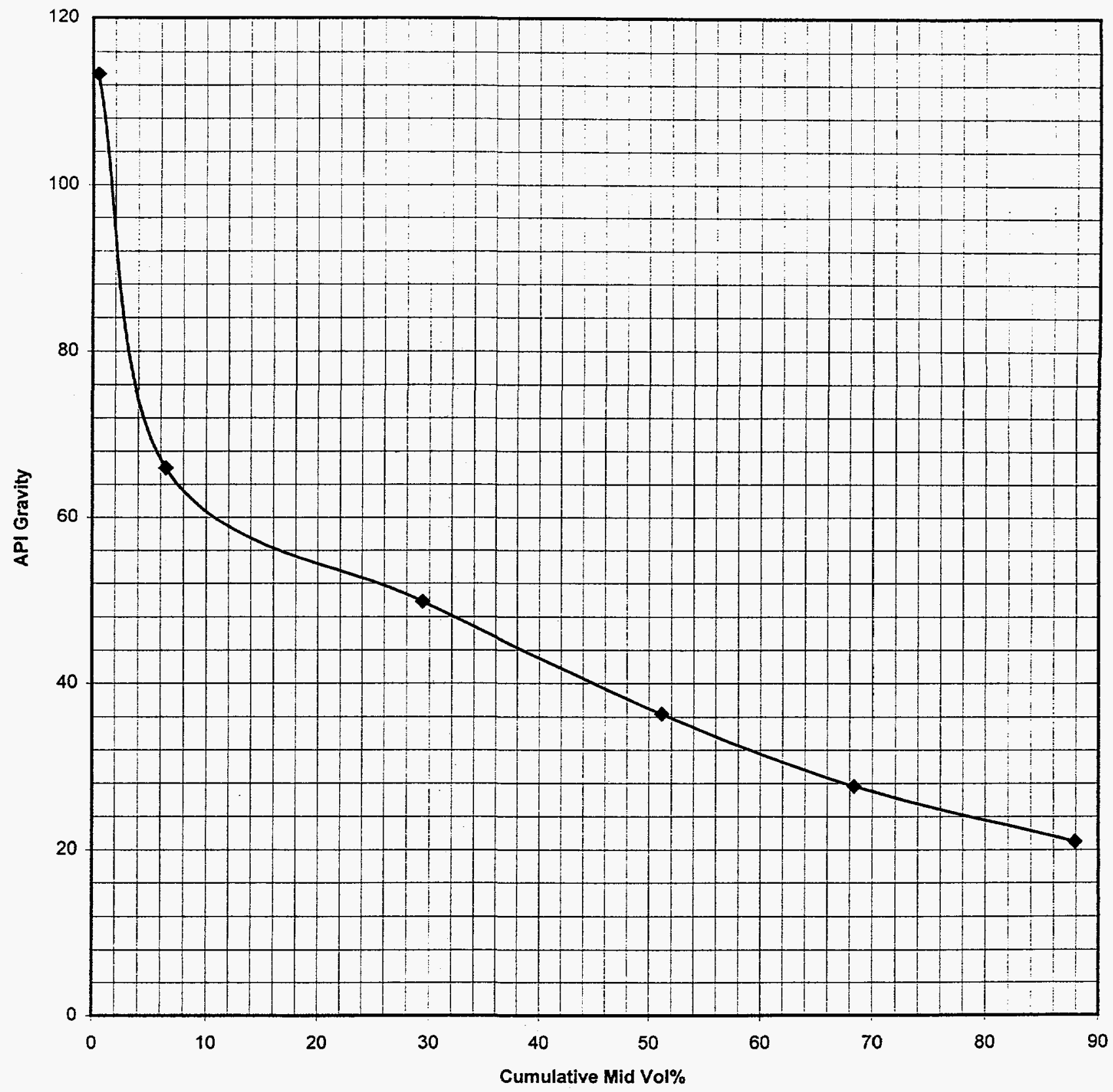




\section{WinAssay ' 95 \\ True Boiling Point Curve \\ Vaporline Temperature v. Cumulative $\mathrm{W} t \%$ Yield}

Sample ID

HTI PB-03-6,7,8 (Charge \#1)

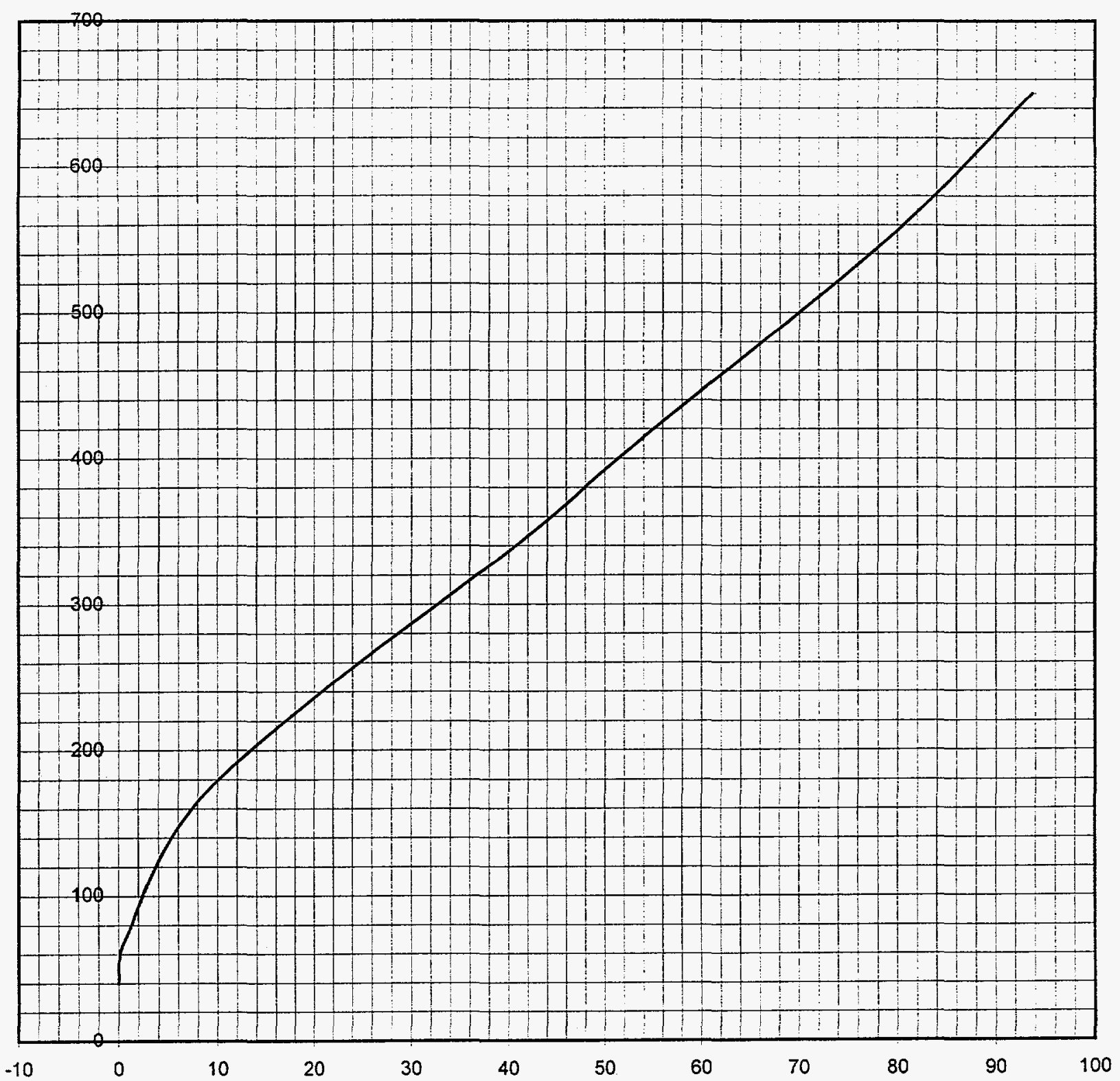


WinAssay ' 95

True Boiling Point Curve vs Cumulative Vol\% Yield

\section{Sample ID}

HTI PB-03-6,7,8 (Charge \#1)

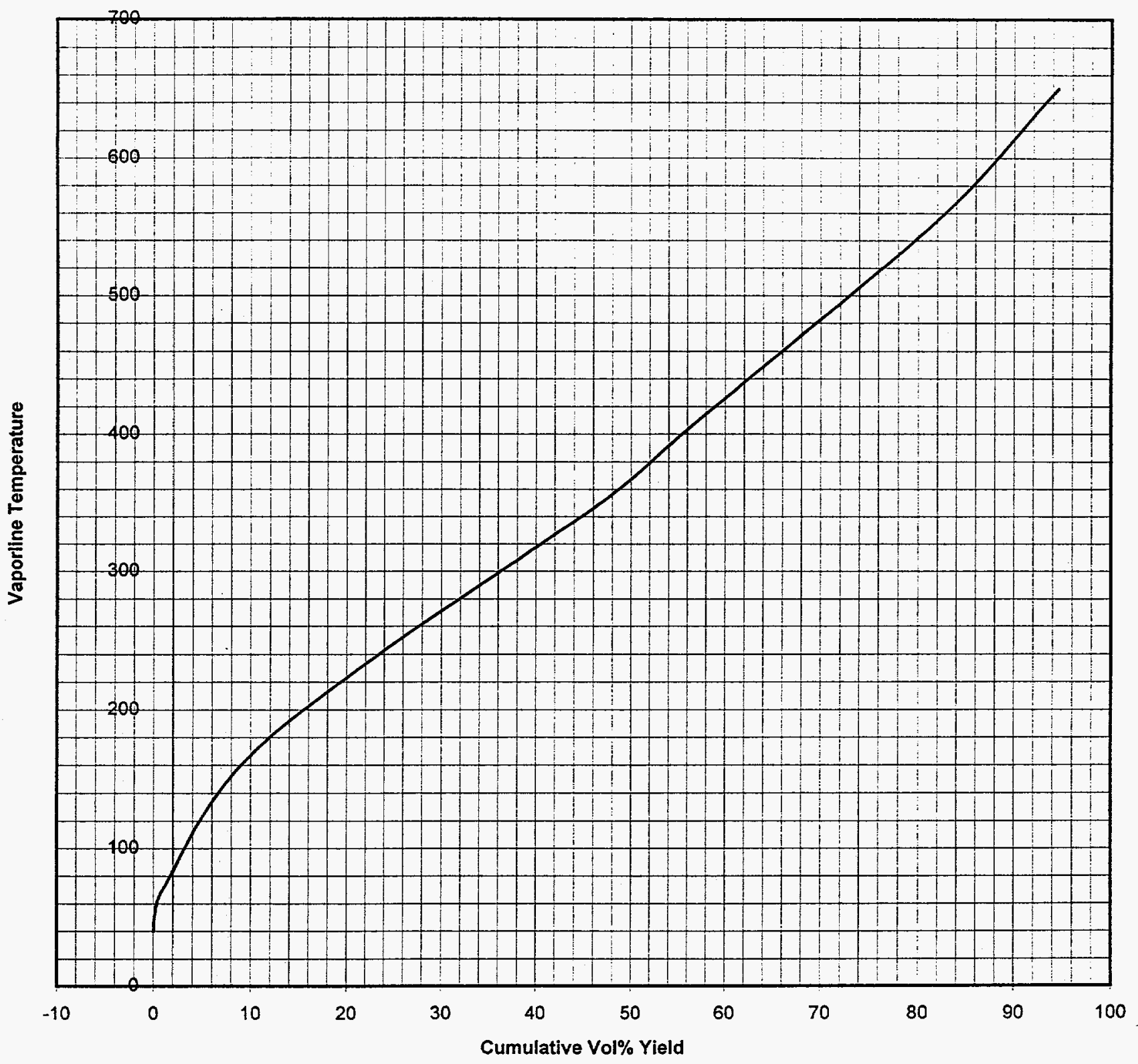


ITS- Caleb Brett Distillation Group

ASTM D2892/D5236 CHARGE INFORMATION

Lab ID:

Client Name:

Sample ID:

Date:

96-005162

Consol lnc.

HTI PB-03-6,7,8 (Charge \#1)

9/25/96

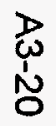

Charge Mass D2892(g):

Charge S.G D2892 (60/60F):

\begin{tabular}{|r|}
\hline 9494.0 \\
\hline 0.8347
\end{tabular}$\quad \begin{aligned} & \text { Water Weight Removed (g): } \\
& \text { Initial Vapor Temp: } \\
& \text { Whole Crude Sulfur Wt\%: }\end{aligned}$

Operator: Robert Kelly

Charge Mass D5236(g):

0.0

Whole Crude Sulfur Wt\%:

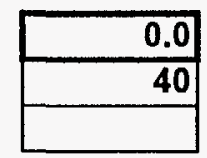

0.0000

Charge S.G. D5236 (60/60F): 
TID: 96-005162-0-HUUS-002-00

CID: CONSULINC

SID: HTI PB-03-6,7,8 CRUDE OIL/IBP-7O F

NID : 51851

Date: 16-SEP-1996

Analyzed: 9/20/96 5:03 PM

Reported: 09-23-1996 11:06:08 Normalized to $100.00 \%$

Components Listed in Chromatographic Order

$\begin{array}{ccl}\text { Min. } & \text { INDEX } & \text { Component } \\ 8.746 & 200.0 & \text { ethane } \\ 9.242 & 294.1 & \text { propylene } \\ 9.298 & 300.0 & \text { propane } \\ 10.322 & 366.3 & \text { i-butane } \\ 10.991 & 391.0 & \text { butene-1 } \\ 11.293 & 400.0 & \text { n-butane } \\ 11.608 & 411.1 & \text { t-butene-2 } \\ 11.701 & 414.2 & 2 \text {-2-dimethylpropane } \\ 12.121 & 427.0 & \text { c-butene-2 } \\ 13.491 & 460.3 & \text { 3-methylbutene-1 } \\ 13.726 & 465.0 & \text { ? } \\ 14.398 & 477.6 & \text { i-pentane } \\ 15.156 & 490.1 & \text { pentene-1 } \\ 15.545 & 495.9 & \text { 2-methylbutene-1 } \\ 15.828 & 500.0 & \text { n-pentane } \\ 16.239 & 509.0 & \text { t-pentene-2 } \\ 16.668 & 517.9 & \text { c-pentene-2 } \\ 16.940 & 523.3 & 2 \text {-methylbutene-2 } \\ 17.781 & 538.9 & 2.2 \text {-dimethylbutane } \\ 18.785 & 555.8 & \text { cyclopentene }\end{array}$

$\begin{array}{rrr}\text { Wt\% } & \text { VOI\% } & \text { Mol\% } \\ 0.027 & 0.046 & 0.053 \\ 0.014 & 0.016 & 0.019 \\ 8.488 & 9.812 & 11.350 \\ 5.925 & 6.153 & 6.011 \\ 0.091 & 0.088 & 0.095 \\ 63.634 & 63.611 & 64.556 \\ 0.206 & 0.197 & 0.216 \\ 0.091 & 0.089 & 0.074 \\ 0.123 & 0.114 & 0.129 \\ 0.007 & 0.006 & 0.006 \\ 0.058 & 0.054 & 0.049 \\ 10.862 & 10.143 & 8.877 \\ 0.036 & 0.032 & 0.030 \\ 0.014 & 0.013 & 0.012 \\ 10.326 & 9.541 & 8.439 \\ 0.044 & 0.040 & 0.037 \\ 0.018 & 0.016 & 0.015 \\ 0.013 & 0.011 & 0.011 \\ 0.004 & 0.003 & 0.003 \\ 0.021 & 0.016 & 0.018\end{array}$


CALEB BRETT HUUSTUN

TID: 96-005162-0-HUUS-002-00

CID: CONSULINC

SID: HTI PB-03-6,7,8 CRUDE OIL/IBP-70 F

NID: 51851

Date: $16-$ SEP-1996

Analyzed: 9/20/96 5:03 PM

Reported: 09-23-1996 11:06:08

Normalized to $100.00 \%$

Composite Report

Totals by Group Type \& Carbon Number (in Weight Percent)

Paraffins: I-paraffins: Aromatics: Naphthenes: Olefins: Total:

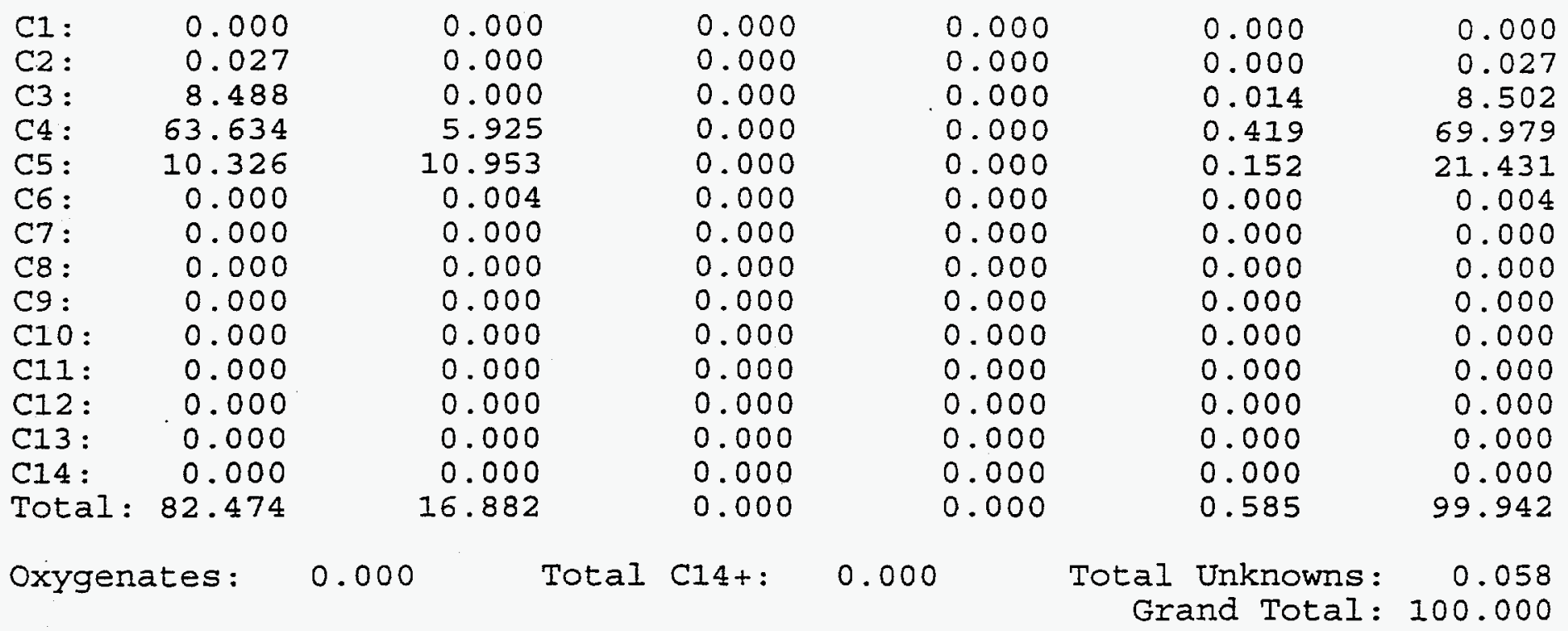

Molecular Weight and Relative Density Data

$\begin{array}{rrr}\text { Group: } & \text { Ave. Mw.: } & \text { Ave. Rel. Density: } \\ \text { C1: } & 0.000 & 0.000 \\ \text { C2: } & 30.070 & 0.340 \\ \text { C3: } & 44.094 & 0.501 \\ \text { C4: } & 58.112 & 0.577 \\ \text { C5: } & 72.134 & 0.623 \\ \text { C6: } & 86.178 & 0.649 \\ \text { C7: } & 0.000 & 0.000 \\ \text { C8: } & 0.000 & 0.000 \\ \text { C9: } & 0.000 & 0.000 \\ \text { C10: } & 0.000 & 0.000 \\ \text { C11: } & 0.000 & 0.000 \\ \text { C12: } & 0.000 & 0.000 \\ \text { C13: } & 0.000 & 0.000 \\ \text { C14: } & 0.000 & 0.000 \\ \text { Sample: } & 58.932 & 0.578\end{array}$

File: $5174 \mathrm{~A} 2 . \mathrm{DHA}$ 
CALEB BRETIT HUUSTUN

TID: 96-0U5 162-0-HUUS-0ن்2-00

CID: CONSOLINC

SID: HTI PB-Ü-6,7,8 CRUDE OIL/IBP-7U E

NID: 51851

Date: $16-$ SEP-1996

Analyzed: 9/20/96 5:03 PM

Reported: 09-23-1996 11:06:08

Normalized to $100.00 \%$

Composite Report

Totals by Group Type \& Carbon Number

(in Volume Percent)

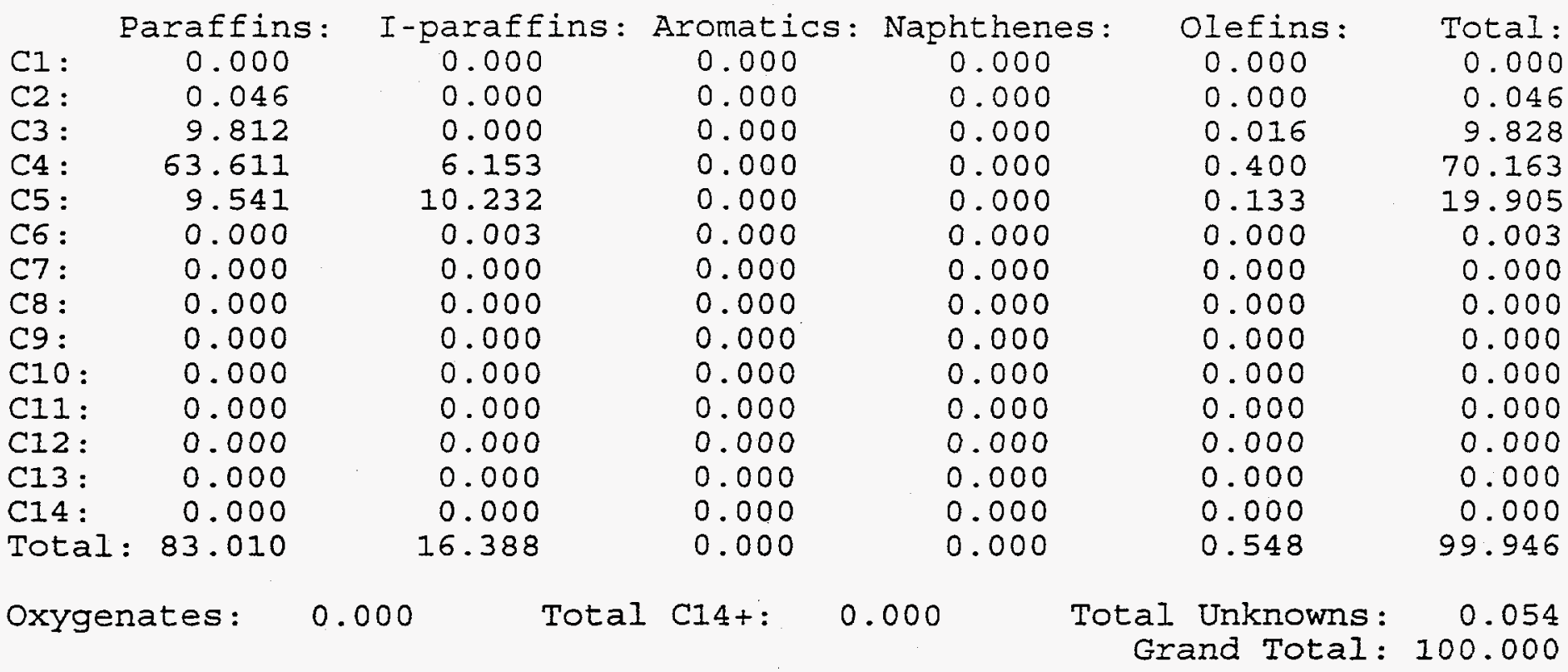

(in Mole Percent)

Paraffins: I-paraffins: Aromatics: Naphthenes: Olefins: Total:

\begin{tabular}{|c|c|c|c|c|c|}
\hline 0.000 & 0.000 & 0.000 & 0.000 & 0.000 & 0.000 \\
\hline 0.053 & 0.000 & 0.000 & 0.000 & 0.000 & 0.053 \\
\hline 11.350 & 0.000 & 0.000 & 0.000 & 0.019 & 11.369 \\
\hline 64.556 & 6.011 & 0.000 & 0.000 & 0.441 & 71.008 \\
\hline 8.439 & 8.951 & 0.000 & 0.000 & 0.128 & 17.518 \\
\hline 0.000 & 0.003 & 0.000 & 0.000 & 0.000 & 0.003 \\
\hline 0.000 & 0.000 & 0.000 & 0.000 & 0.000 & 0.000 \\
\hline 0.000 & 0.000 & 0.000 & 0.000 & 0.000 & 0.000 \\
\hline 0.000 & 0.000 & 0.000 & 0.000 & 0.000 & 0.000 \\
\hline 0.000 & 0.000 & 0.000 & 0.000 & 0.000 & 0.000 \\
\hline 0.000 & 0.000 & 0.000 & 0.000 & 0.000 & 0.000 \\
\hline 0.000 & 0.000 & 0.000 & 0.000 & 0.000 & 0.000 \\
\hline 0.000 & 0.000 & 0.000 & 0.000 & 0.000 & 0.000 \\
\hline 0.000 & 0.000 & 0.000 & 0.000 & 0.000 & 0.000 \\
\hline 84.397 & 14.965 & 0.000 & 0.000 & 0.588 & 99.951 \\
\hline
\end{tabular}

Oxygenates: $\quad 0.000 \quad$ Total C14t: 0.000

Total Unknowns: 0.049 Grand Total: 100.000

File: $5174 \mathrm{~A} 2 . \mathrm{DHA}$ 
CALEB BRETT HUUSTUN

TID: 95-0U516Z2-U-HUUS-UU2-UO

CID: CONSULINC

SID: $\operatorname{HTI} \mathrm{PB}-03-6,7,8$ CRUUE OIL/IBP-7U $E$

NID: 51851

Date: $16-5 E P-1996$

Analyzed: 9/20/96 5:03 PM

Reported: 09-23-1996 11:06:08

Normalized to $100.00 \%$

Boiling Point Distribution Data

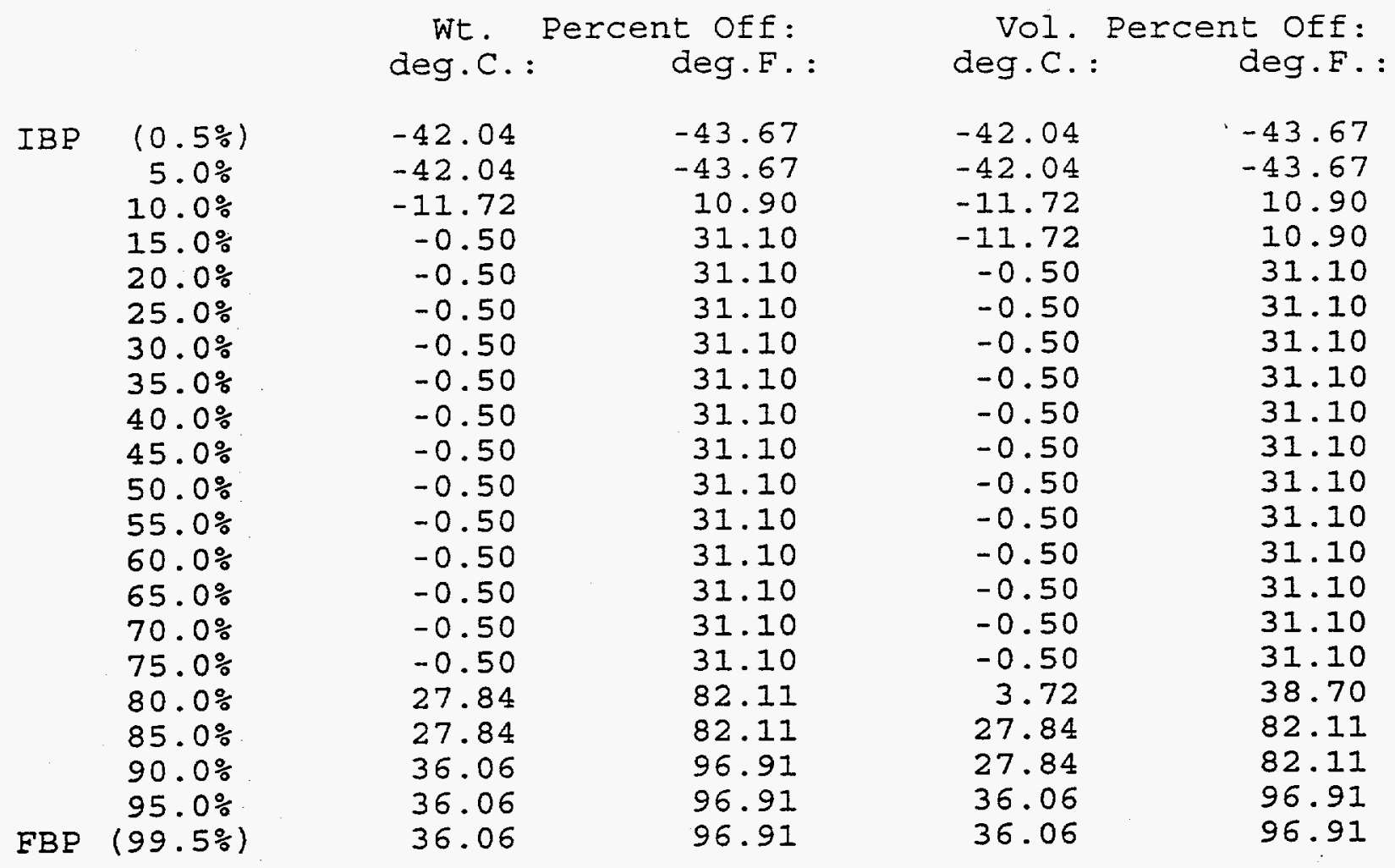

Research Octane Number $=107.33$

(Calculated from Individual Component VaIues)

Contribution to Total by:

$\begin{array}{lr}\text { Paraffins: } & 88.70 \\ \text { Iso-paraffins: } & 17.97 \\ \text { Aromatics: } & 0.00 \\ \text { Naphthenes: } & 0.00 \\ \text { Olefins: } & 0.61 \\ \text { Oxygenates: } & 0.00\end{array}$

File: $5174 \mathrm{~A} 2 . \mathrm{DHA}$ 


\section{WinAssay ' 95}

Version 1.00

Final Reports

Client Name: $\quad$ Consol Inc.

Sample ID:

HTI PB-03-6,7,8 (Charge \#2)

Laboratory ID: $\quad \underline{96-005162}$

Date:

$\underline{9 / 25 / 96}$

Operator: $\quad$ Robert Kelly 


\begin{tabular}{|c|c|c|c|c|c|c|c|c|c|c|}
\hline \multicolumn{11}{|c|}{ Distillation Summary Report } \\
\hline \multicolumn{2}{|c|}{$\begin{array}{l}\text { Prepared For: } \\
\text { Sample ID: } \\
\text { Date: }\end{array}$} & \multicolumn{9}{|c|}{$\begin{array}{l}\text { Consol Inc. } \\
\text { HTI PB-03-6,7,8 (Charge \#2) } \\
9 / 25 / 96\end{array}$} \\
\hline $\begin{array}{r}\text { Cut Temp } \\
\text { TO } \\
\end{array}$ & Degrees F & $\begin{array}{l}\text { DUMP } \\
\text { WT(g) } \\
\end{array}$ & $\begin{array}{l}\text { Specific } \\
\text { Gravity }\end{array}$ & MLS & $\begin{array}{c}\text { LIQ } \\
\text { VOL\% } \\
\end{array}$ & $\begin{array}{c}\text { CUM. LIQ } \\
\text { VOL\%. }\end{array}$ & "WT\% & $\begin{array}{l}\text { CUNI } \\
\text { WT } \% \\
\end{array}$ & $\begin{array}{c}\text { API } \\
\text { GRAVITY } \\
\end{array}$ & $\begin{array}{c}\text { MID } \\
\text { LIQ VOL\% } \\
\end{array}$ \\
\hline \multicolumn{11}{|c|}{ ASTM D2892 Distillation Yields } \\
\hline IBP & 70 & 58.30 & 0.5880 & 99.15 & 0.94 & 0.94 & 0.66 & 0.66 & 109.15 & 0.47 \\
\hline 70 & 180 & 874.20 & 0.7185 & 1216.70 & 11.51 & 12.45 & 9.91 & 10.57 & 65.44 & 6.70 \\
\hline 180 & 350 & 2866.60 & 0.7792 & 3678.90 & 34.82 & 47.27 & 32.50 & 43.07 & 50.10 & 29.86 \\
\hline 350 & 400 & 756.20 & 0.8444 & 895.55 & 8.48 & 55.74 & 8.57 & 51.65 & 36.07 & 51.51 \\
\hline 400 & 550 & 2426.70 & 0.8899 & 2726.94 & 25.81 & 81.55 & 27.51 & 79.16 & 27.51 & 68.65 \\
\hline 550 & 650 & 1282.60 & 0.9280 & 1382.11 & 13.08 & 94.63 & 14.54 & 93.70 & 20.98 & 88.09 \\
\hline $650+$ & & 555.40 & 0.9260 & 599.78 & 5.68 & 100.31 & 6.30 & 100.00 & 21.31 & \\
\hline
\end{tabular}

离

Loss (Grams): 17.8 (0.20 Wt.\%)

Distribution: (2/3) $11.9 \mathrm{~g}$ to IBP-70 F

(1/3) $5.9 \mathrm{~g}$ to 70-180 F 


\section{WinAssay '95 Quality Control Applications}

\section{Cum. Mid Vol\% v. API Gravity}

HTI PB-03-6,7,8 (Charge \#2)

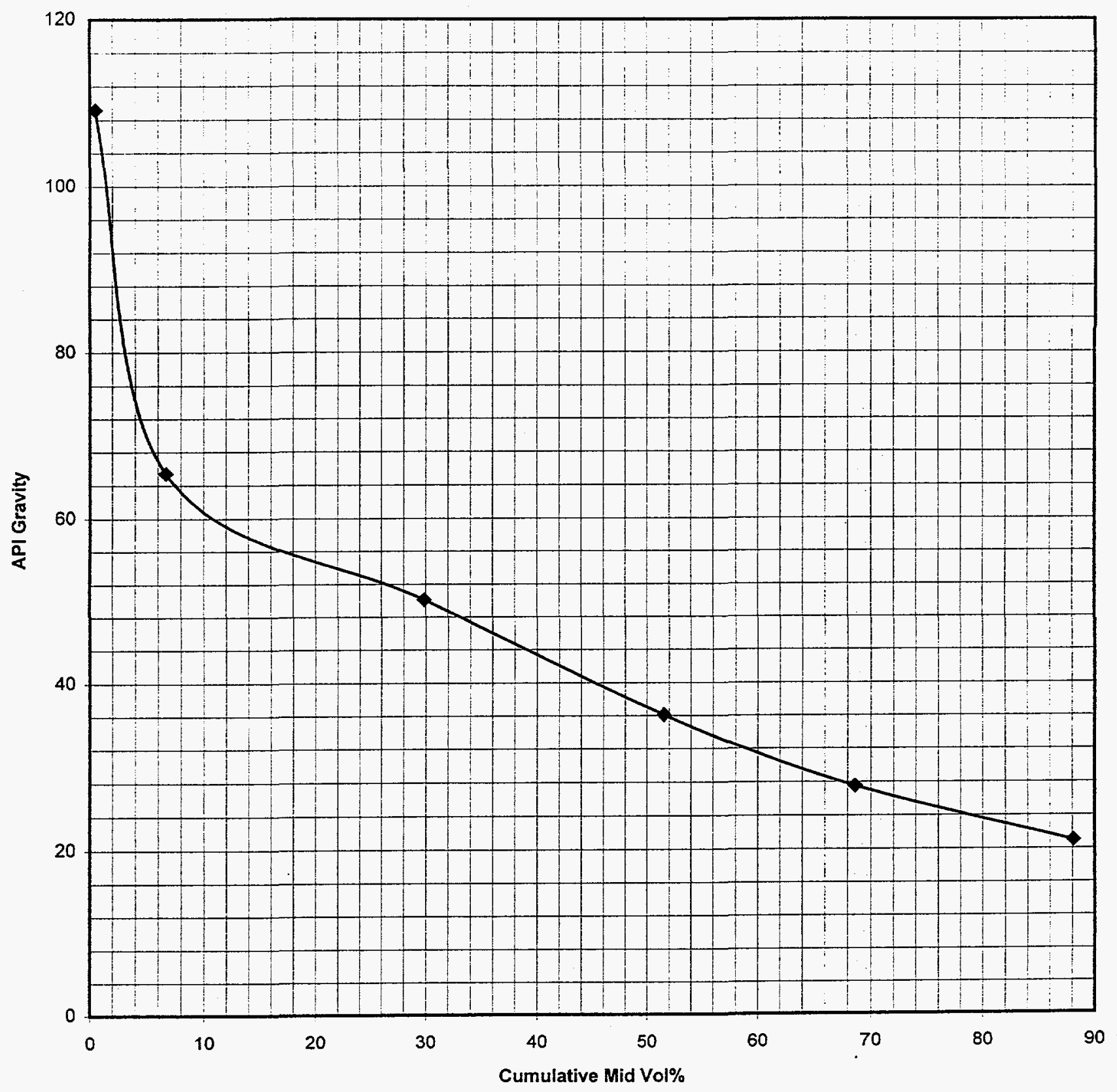




\section{WinAssay ' 95 \\ True Boiling Point Curve \\ Vaporline Temperature v. Cumulative $\mathrm{Wt} \%$ Yield}

Sample ID

HTI PB-03-6,7,8 (Charge \#2)

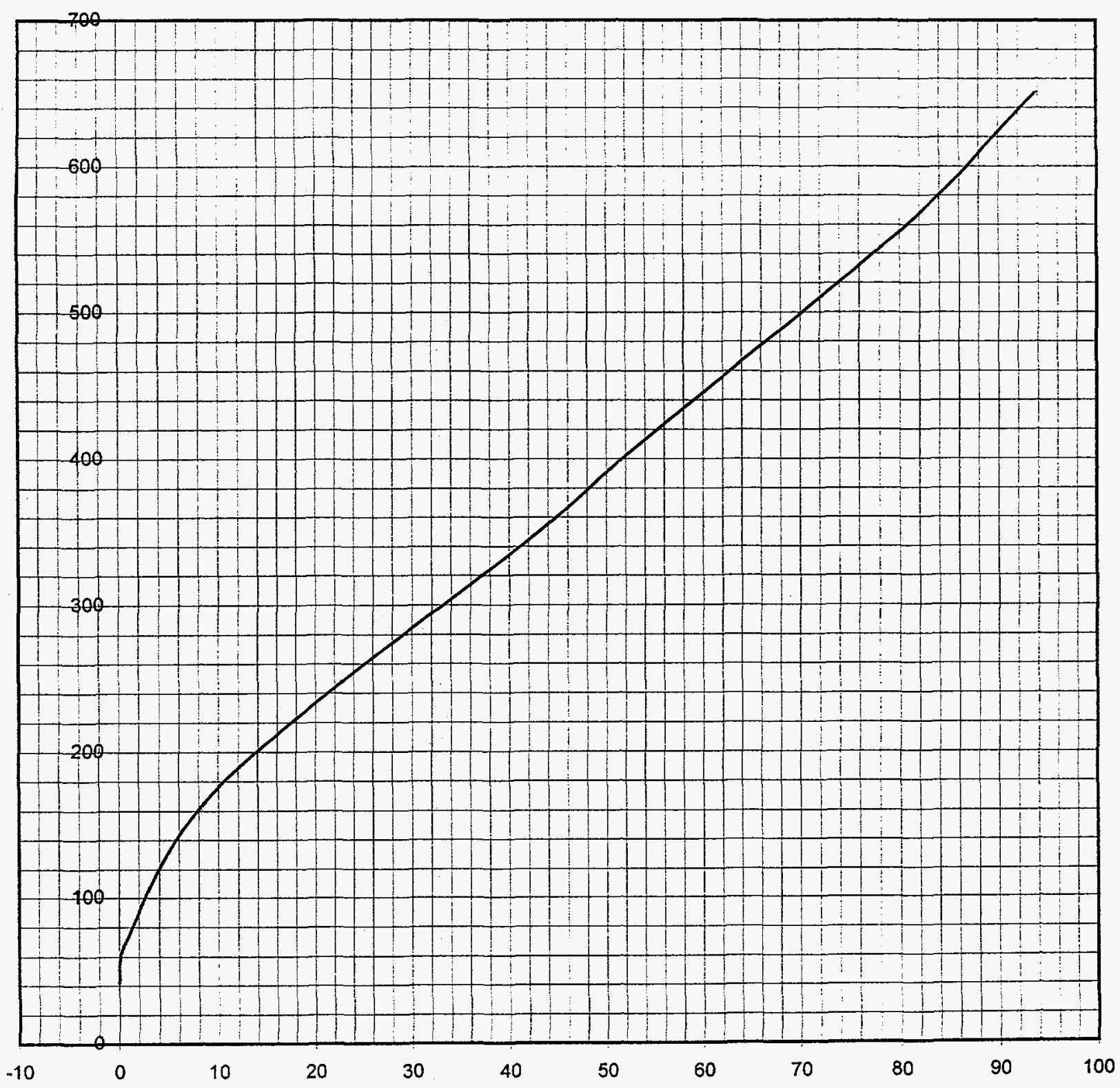




\section{WinAssay ' 95 \\ True Boiling Point Curve vs Cumulative Vol\% Yield}

Sample ID

HTI PB-03-6,7,8 (Charge \#2)

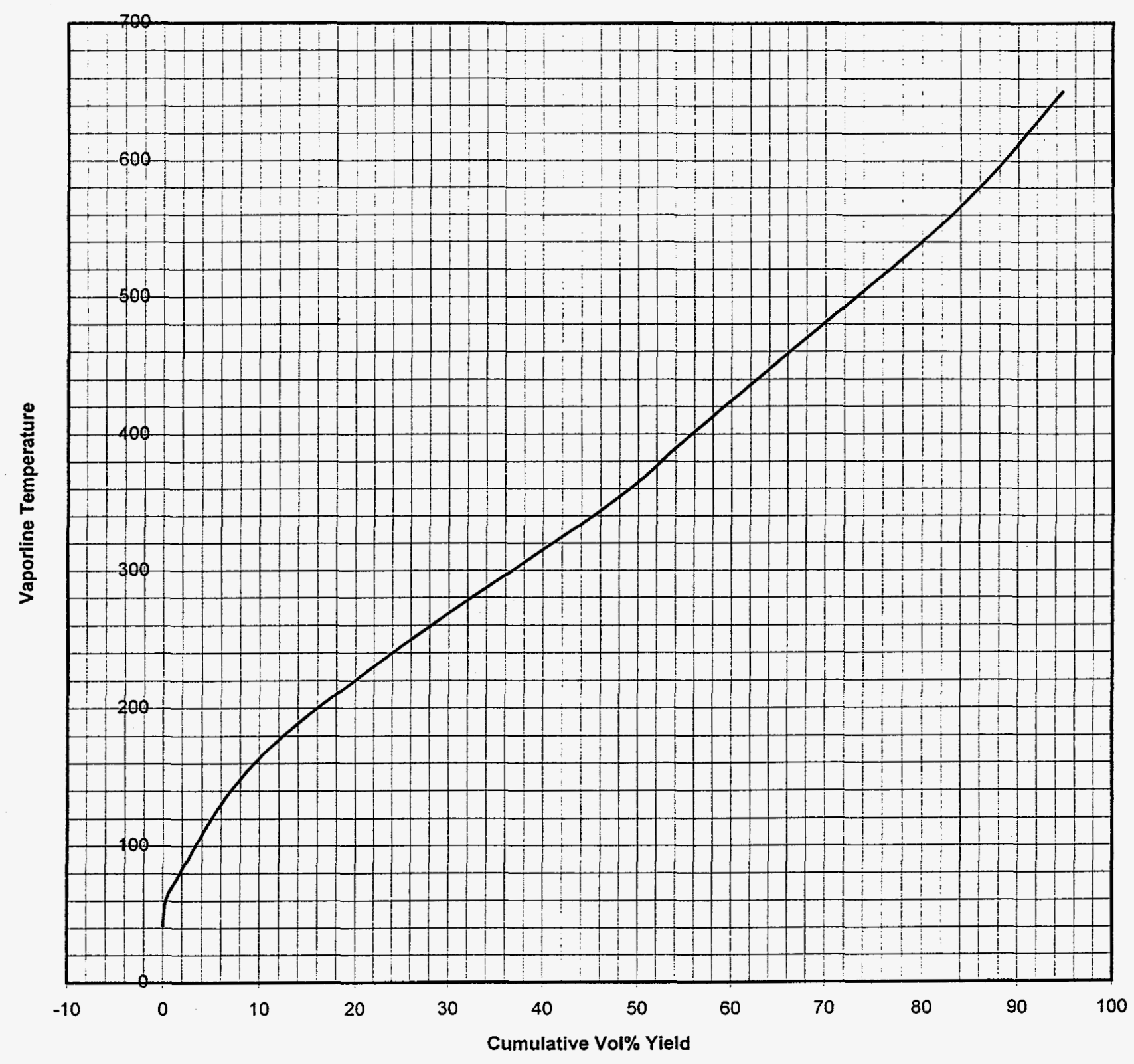


ITS- Caleb Brett Distillation Group

ASTM D2892/D5236 CHARGE INFORMATION

Lab ID:

96-005162

Client Name:

Sample ID:

Date:

HTI PB-03-6,7,8 (Charge \#2)

9/25/96

\author{
Operator: Robert Kelly
}

Charge Mass D2892(g):

Charge S.G D2892 (60/60F):

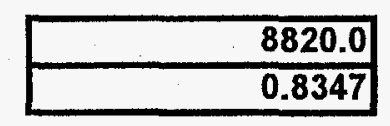

Water Weight Removed (g):

Initial Vapor Temp:

Whole Crude Sulfur Wt\%:

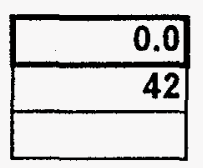

Charge Mass D5236(g):

Charge S.G. D5236 (60/60F):

0.0

0.0000 


\section{GALEB BRET"T HUUSTUN}

TID: $90-005162-0-H 005-002-00$

CID: CUNSULINC

SID: HTI PB-03-6,7,8 CRUDE OIL/IBP-7U $\mathrm{F}$

NID: 51851

Date: $16-5 E P-1996$

Analyzed: 9/20/96 6:06 PM Reported: 09-23-1996 11:09:47 Normalized to $100.00 \%$

Components Listed in Chromatographic order

\begin{tabular}{cll} 
Min. & INDEX & \multicolumn{1}{c}{ Component } \\
9.308 & 300.0 & propane \\
10.324 & 366.0 & i-butane \\
10.994 & 391.0 & butene-1 \\
11.293 & 400.0 & n-butane \\
11.616 & 411.4 & t-butene-2 \\
11.708 & 414.4 & 2, - dimethylpropane \\
12.125 & 427.0 & c-butene-2 \\
13.497 & 460.2 & 3-methylbutene-1 \\
13.740 & 465.1 & ? \\
14.409 & 477.5 & i-pentane \\
15.161 & 489.9 & pentene-1 \\
15.554 & 495.8 & 2-methylbutene-1 \\
15.850 & 500.0 & n-pentane \\
16.246 & 508.7 & ? \\
16.674 & 517.6 & ? \\
16.945 & 522.9 & 2-methylbutene-2 \\
17.787 & 538.6 & ? \\
18.797 & 555.7 & Cyclopentene \\
19.435 & 565.6 & cyclopentane \\
19.582 & 567.8 & 4 -methyl-c-pentene-2 \\
19.882 & 572.2 & 2-methylpentane \\
20.776 & 584.7 & 3-methylpentane \\
21.973 & 600.0 & n-hexane \\
23.884 & 625.5 & methylcyclopentane
\end{tabular}

$\begin{array}{rrr}\text { Wt\% } & \text { VOI\% } & \text { MoI\% } \\ 0.213 & 0.251 & 0.295 \\ 6.759 & 7.137 & 7.083 \\ 0.108 & 0.107 & 0.117 \\ 69.245 & 70.395 & 72.569 \\ 0.246 & 0.240 & 0.267 \\ 0.111 & 0.110 & 0.094 \\ 0.149 & 0.141 & 0.162 \\ 0.006 & 0.006 & 0.005 \\ 0.033 & 0.031 & 0.029 \\ 9.621 & 9.137 & 8.123 \\ 0.032 & 0.029 & 0.027 \\ 0.011 & 0.010 & 0.009 \\ 11.015 & 10.351 & 9.300 \\ 0.047 & 0.044 & 0.039 \\ 0.019 & 0.017 & 0.016 \\ 0.014 & 0.012 & 0.012 \\ 0.010 & 0.008 & 0.008 \\ 0.011 & 0.008 & 0.009 \\ 1.039 & 0.820 & 0.902 \\ 0.050 & 0.044 & 0.036 \\ 0.422 & 0.380 & 0.298 \\ 0.221 & 0.196 & 0.156 \\ 0.361 & 0.322 & 0.255 \\ 0.259 & 0.203 & 0.187\end{array}$

File: $516482 . \mathrm{DHA}$

Sample: $96-5164-2 b$

p. 1 


\section{CALEB BRETT HUUSTON}

TID: 96-005162-0-HUUS-002-00

CID: CONSOLINC

SID: HTI PB-O3-6,7,8 CRUUE OIL/IBP-70 E

NID: 51851

Date: $16-5 \mathrm{EP}-1996$

Analyzed: 9/20/96 6:06 PM

Reported: 09-23-1996 11:09:47

Normalized to $100.00 \%$

Composite Report
Totals by Group Type \& Carbon Number
(in Weight Percent)

Paraffins: I-paraffins: Aromatics: Naphthenes: Olefins: Total:

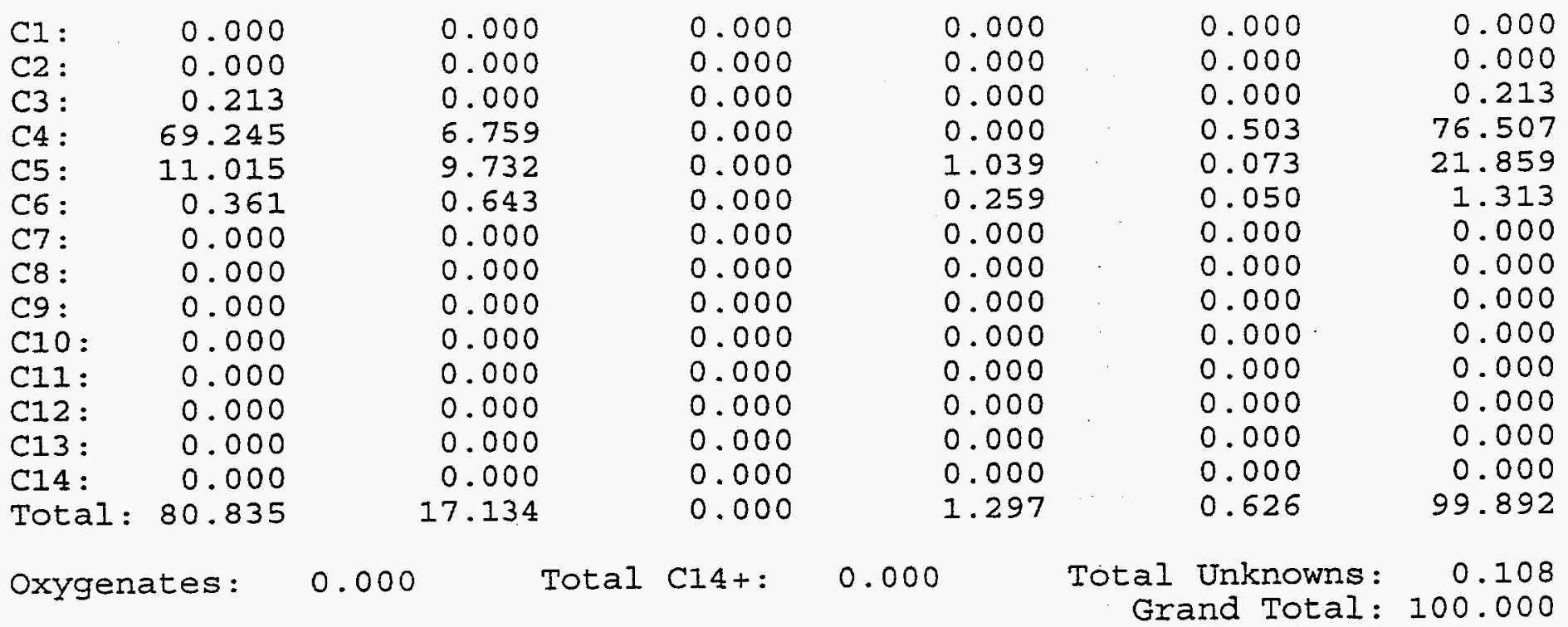

Molecular Weight and Relative Density Data

$\begin{array}{crc}\text { Group: } & \text { Ave. Mw.: } & \text { Ave. Rel. Density: } \\ \text { C1: } & 0.000 & 0.000 \\ \text { C2: } & 0.000 & 0.000 \\ \text { C3: } & 44.097 & 0.501 \\ \text { C4: } & 58.110 & 0.577 \\ \text { C5: } & 72.045 & 0.628 \\ \text { C6: } & 85.695 & 0.675 \\ \text { C7: } & 0.000 & 0.000 \\ \text { C8: } & 0.000 & 0.000 \\ \text { C9: } & 0.000 & 0.000 \\ \text { C10: } & 0.000 & 0.000 \\ \text { C11: } & 0.000 & 0.000 \\ \text { C12: } & 0.000 & 0.000 \\ \text { C13: } & 0.000 & 0.000 \\ \text { C14: } & 0.000 & 0.000 \\ \text { Total Sample: } & 60.848 & 0.588\end{array}$

File: $5164 \mathrm{~B} 2 . \mathrm{DHA}$ 
CALEB BRETT HUUSTON

TID: 95-005162-0-HOUS-002-00

CID: CONSULINC

SID: HTI PB-03-6,7,8 CRUDE OIL/IBP-7O E

NID: 51851

Date: 16-SEP-1996

Analyzed: 9/20/96 6:06 PM

Reported: 09-23-1996 11:09:47

Normalized to $100.00 \%$

Composite Report

Totals by Group Type \& Carbon Number (in Volume Percent)

$\begin{array}{cc} & \text { Paraffins: } \\ \text { C1: } & 0.000 \\ \text { C2: } & 0.000 \\ \text { C3: } & 0.251 \\ \text { C4: } & 70.395 \\ \text { C5: } & 10.351 \\ \text { C6 : } & 0.322 \\ \text { C7: } & 0.000 \\ \text { C8 : } & 0.000 \\ \text { C9: } & 0.000 \\ \text { C10: } & 0.000 \\ \text { C11: } & 0.000 \\ \text { C12: } & 0.000 \\ \text { C13: } & 0.000 \\ \text { C14: } & 0.000 \\ \text { T0Ea1 } & 81.319\end{array}$

Total: 81.319
I-paraffins:
0.000

0.000
0.000

0.000

7.137

9.248

0.576

0.000

0.000

0.000

0.000

0.000

0.000

0.000

0.000

16.961
Aromatics: Naphthenes:

0.000

0.000

0.000

0.000

0.000

0.000

0.000

0.000

0.000

0.000

0.000

0.000

0.000

0.000

0.000
0.000

0.000

0.000

0.000

0.820

0.203

0.000

0.000

0.000

0.000

0.000

0.000

0.000

0.000

1.023
Olefins:

0.000

0.000

0.000

0.488

0.065

0.044

0.000

0.000

0.000

0.000

0.000

0.000

0.000

0.000

0.596
Total:

0.000

0.000

0.251

78.020

20.483

I. 145

0.000

0.000

0.000

0.000

0.000

0.000

0.000

0.000

99.899

Oxygenates: $\quad 0.000 \quad$ Total Cl4t: 0.000

Total Unknowns:

0.101 Grand Total: 100.000

(in Mole Percent)
I-paraffins: Aromatics

$\begin{array}{lc}\text { C1: } & 0.000 \\ \text { C2: } & 0.000 \\ \text { C3: } & 0.295 \\ \text { C4: } & 72.569 \\ \text { C5: } & 9.300 \\ \text { C6: } & 0.255 \\ \text { C7: } & 0.000 \\ \text { C8: } & 0.000 \\ \text { C9: } & 0.000 \\ \text { C10: } & 0.000 \\ \text { C11: } & 0.000 \\ \text { C12: } & 0.000 \\ \text { C13: } & 0.000 \\ \text { C14: } & 0.000 \\ \text { Total : } & 82.418 \\ \text { Oxygenates: } & 0.00 \\ & \end{array}$

0.000
0.000
0.000

0.000

0.000

0.000

7.083

0.000

8.217

0.000

0.454

0.000

0.000

0.000

0.000

0.000

0.000

0.000

0.000

0.000

0.000

0.000

0.000

0.000

0.000

0.000

0.000

0.000

15.754

0.000

Olefins :

Total:

0.000

0.000

0.000

0.000

0.000

0.000

0.295

0.000

0.000

80.198

0.902

0.546

0.063

0.036

18.482

0.187

0.000

0.933

0.000

0.000

0.000

0.000

0.000

0.000

0.000

0.000

0.000

0.000

0.000

0.000

0.000

0.000

0.000

0.000

0.000

0.000

0.000

1.089

0.646

99.908

Oxygenates: $0.000 \quad$ Total C14t: 0.000

Total Unknowns: 0.092 Grand Total: 100.000 
CALEB BRETIT HUUSTON

TID: 96-005162-0-HUUS-00'2-00

CID: CONSULINC

SID: HTI PB-U3-6,7,8 CRUDE OIL/IBP-70 E

NID : 51851

Date: $16-5 E P-1996$

Analyzed: 9/20/96 6:06 PM

Reported: 09-23-1996 11:09:47

Normalized to $100.00 \%$

Boiling Point Distribution Data

$\begin{array}{ccccc}\text { IBP }(0.5 \%) & -11.72 & 10.90 & -11.72 & 10.90 \\ 5.0 \% & -11.72 & 10.90 & -11.72 & 10.90 \\ 10.0 \% & -0.50 & 31.10 & -0.50 & 31.10 \\ 15.0 \% & -0.50 & 31.10 & -0.50 & 31.10 \\ 20.0 \% & -0.50 & 31.10 & -0.50 & 31.10 \\ 25.0 \% & -0.50 & 31.10 & -0.50 & 31.10 \\ 30.0 \% & -0.50 & 31.10 & -0.50 & 31.10 \\ 35.0 \% & -0.50 & 31.10 & -0.50 & 31.10 \\ 40.0 \% & -0.50 & 31.10 & -0.50 & 31.10 \\ 45.0 \% & -0.50 & 31.10 & -0.50 & 31.10 \\ 50.0 \% & -0.50 & 31.10 & -0.50 & 31.10 \\ 55.0 \% & -0.50 & 31.10 & -0.50 & 31.10 \\ 60.0 \% & -0.50 & 31.10 & -0.50 & 31.10 \\ 65.0 \% & -0.50 & 31.10 & -0.50 & 31.10 \\ 70.0 \% & -0.50 & 31.10 & -0.50 & 31.10 \\ 75.0 \% & -0.50 & 31.10 & -0.50 & 31.10 \\ 80.0 \% & 27.84 & 82.11 & 27.84 & 82.11 \\ 85.0 \% & 27.84 & 82.11 & 27.84 & 82.11 \\ 90.0 \% & 36.06 & 96.91 & 36.06 & 96.91 \\ 95.0 \% & 36.06 & 96.91 & 36.06 & 96.91 \\ \text { FBP }(99.5 \%) & 68.73 & 155.71 & 68.73 & 155.71\end{array}$

Research Octane Number $=105.62$

(Calculated from Individual Component Values)

$\begin{array}{lc}\text { Contribution to Total by: } \\ \text { Paraffins: } & 85.41 \\ \text { Iso-paraffins: } & 18.33 \\ \text { Aromatics: } & 0.00 \\ \text { Naphthenes: } & 1.15 \\ \text { Olefins: } & 0.65 \\ \text { Oxygenates: } & 0.00\end{array}$

File: 5164B2.DHA 
Consol, Inc.

$11 / 04 / 96$

4000 Brownsville Road

Library, PA 15129-9566

Attention: R.A. Winschell

Reference: Crude Assay on "HTI PB-03-9, 10, 11" received in September 1996

Mr. Winschell,

The following results are based on a composite of (2) separate distillations performed on this sample. The Distillation Data Report provides a breakdown of the separate distillations. Should you have any questions concerning this report, you may contact me at (713) 844-3311, or by fax at (713) 844-3330.

Sincerely,

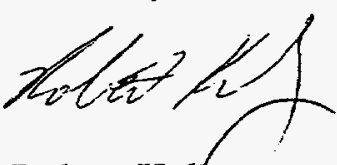

Robert Kelly

Distillation Manager 


\section{Inchcape Testing Services}

Caleb Brett

November 12, 1996

Houston, Texas

Our Reference : $\mathrm{HO} / 96-005170$

Your Reference : PO\# 01-001-033668

Consol, Inc.

Research \& Development

4000 Brownsville Road

Library, PA 15129-9566

ATTN: R.A. Winschel

Reference: To perform "CRUDE ASSAY" on "HT1 PB-03-9,10,11" received in September 1996.

Dear Mr. Winschel:

Please find enclosed the original report on the above referenced submitted sample(s) and our invoice for services rendered.

Should you have any questions regarding this report, please do not hesitate to contact us at your convenience.

We trust you find all in order and thank you for requesting our services.

Very Truly Yours,

INCHCAPE TESTING SERVICES

CALEB BRETT U.S.A.

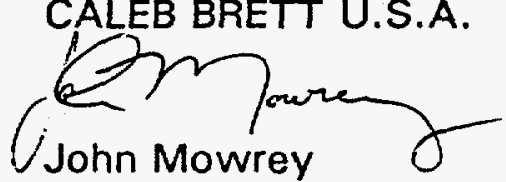

Laboratory Manager

Enclosures:

$\mathrm{JM} / \mathrm{rm}$ 


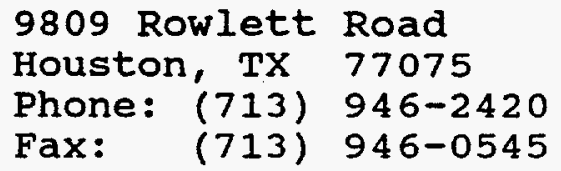

Your Ref: PO\# 01-001-033668

\section{Laboratory Report No. 96-005170-0-HOUS; 1}

Consol, Inc.

4000 Brownsville Road

Library, PA 15129-9566

For the Attention of R.A. Winschel

SAMPLE DETAILS: 8 Sample(s) received on 16-SEP-1996

SOURCE : Consol, Inc.

DESCRIPTION . :

TAB REF

HTI PB-03-9, 10, 11 CRUDE OIL

Sample As Received

IBP-70 Deg. F

70-180 Deg. F

180-350 Deg. F

350-400 Deg. F

400-550 Deg. F

550-650 Deg. F

$650+$ Deg. F

$001-00$

$002-00$

$003-00$

004-00

005-00

006-00

007-00

008-00

\section{CONTAINERS}

RESULTS
5 Gallon Can

SEE ATTACHED SHEETS
SEALS : NONE

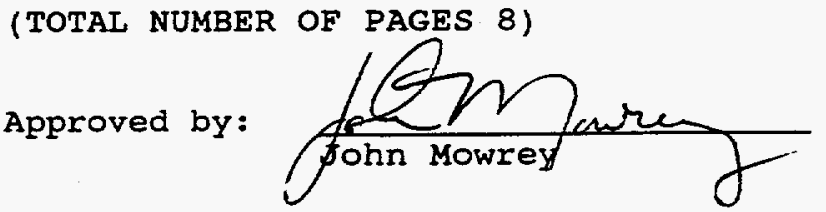


Caleb Brett

Laboratory Report No. 96-005170-0-Hous; 1 - Page 2 of 8

Sample ID

96-005170-0-kOUS-001-00

Test

API Gravity e $60 / 60 \mathrm{~F}$

Specific Gravity a $60 / 60 \mathrm{~F}$

Carbon

Hydrogen

Sulfur Content

Total Nitrogen

Methanol

Ethanol

t-Butanol

Iso-Propanol

n-Propanol

Sec-Butanol

Iso-Butanol

n-Butanol

MTBE

ETBE

DIPE

TAME

t-Pentanol

Total Oxygenates

Oxygen

Ash Content for Digestion

Vanadium

Nickel

Iron

Copper

Freezing Point

Microcarbon Residue

N-Heptane Insolubles

Boiling Point Distribution

\section{Description}

HTI PB-03-9, 10, 11 CRUDE OIL

Sample As Received

Method

D4052

D4052

Wt. \&

Wt. \%

Wt. \&

ppm

Wt. \&

Wt. \&

Wt. \&

Wt. \&

Wt. \&

Wt. 8

Wt. \&

Wt. \&

Wt. \&

Wt. \%

Wt. \&

Wt. \&

Wt. \&

Wt. 8

Wt. \&

Wt. \&

ppm

ppm

ppm

ppm

Deg - F

Wt. o

Wt. \&
D5291

D5291

D4294

D4629

D4815

D4815

D4815

D4815

D4815

D4815

D4815

D4815

D4815

D4815

D4815

D 4815

D4815

D4815

By Difference

D482

ICP

ICP

ICP

ICP

D2386

D4530

D3279

D5307
$001-00$

24.1

0.9091

83.53

10.99

0.31

6716.0

$<0.01$

$<0.01$

$<0.01$

$<0.01$

$<0.01$

$<0.01$

$<0.01$

$<0.01$

$<0.01$

$<0.01$

$<0.01$

$<0.01$

$<0.01$

$<0.01$

4.49

0.004

$<0.1$

$<0.1$

17.7

0.1

Too Dark

$<0.1$

0.09

See Attached
Sample ID

96-005170-0-HOUS-002-00

Test

API Gravity a 60/60 F(Charge \#1)

API Gravity e $60 / 60$ F(Charge \#2)

Specific Gravity e 60/60 F (Charge \#1)

Specific Gravity e $60 / 60 \mathrm{~F}$ (Charge \#2)

DHA

\section{Description}

HTI PB-03-9, 10, 11 CRUDE OIL

IBP-70 Deg. F
Method

$002-00$

G.C.

G.C.

G.C.

G.C.

G.C.
109.2

113.3

0.5880

0.5780

see Attached 


\section{Inchcape Testing Services}

Caleb Brett

Laboratory Report No. 96-005170-0-HoUs; 1 - Page 3 of 8

Sample ID

96-005170-0-HOUS-003-00

Test

API Gravity e $60 / 60$ F Specific Gravity e 60/60 F Carbon

Hydrogen

Sulfur Content

Total Nitrogen

Vapor Pressure

Paraffins

Olefins

Naphthenes

Aromatics

Total N \& A

Benzene Content

Total Acid Number

Corrosion 3 hrs a 122 F

Existent Gum

Oxidation stability

Research Octane Number

Motor Octane Number

Initial Boiling Point

e 58 Evaporated

(e) 10\% Evaporated

e 20\% Evaporated

e 30\% Evaporated

C 40\% Evaporated

e $50 \%$ Evaporated

a 60\% Evaporated

e 708 Evaporated

a 80\% Evaporated

e 908 Evaporated

a $95 \%$ Evaporated

Final Boiling point

Recovery

Residue

Loss
Description

HTI PB-03-9, 10, 11 CRUDE OIL

70-180 Deg. F

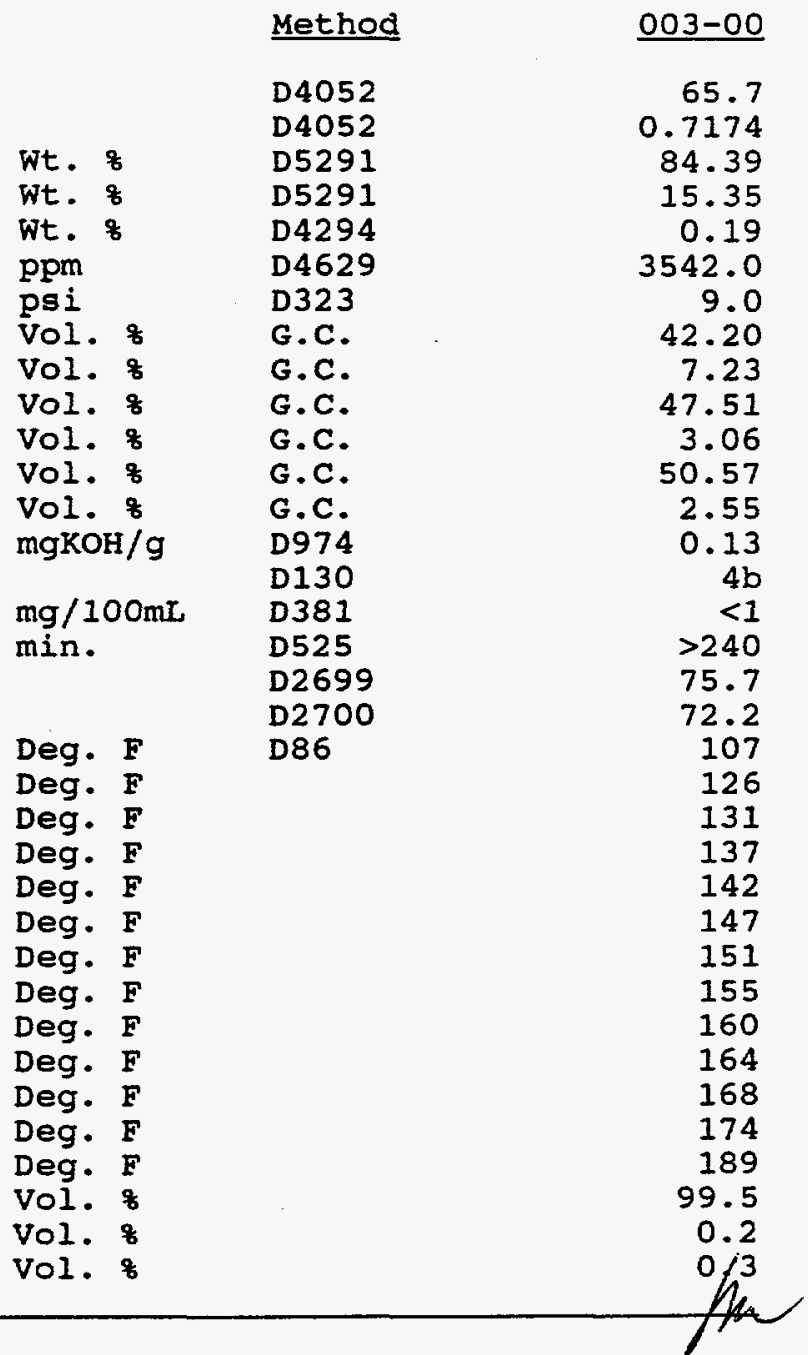


Sample ID

96-005170-0-HOUS-004-00

Test

API Gravity e $60 / 60 \mathrm{~F}$

Specific Gravity e $60 / 60 \mathrm{~F}$

Carbon

Hydrogen

Sulfur content

Total Nitrogen

Mercaptan Sulfur Content

Vapor Pressure

Paraffins

Olefins

Naphthenes

Aromatics

Total N \& A

Corrosion 3 hrs a 122 F

Existent Gum

Oxidation stability

Research Octane Number

Motor Octane Number

Initial Boiling Point

e 58 Evaporated

a 108 Evaporated

a 208 Evaporated

a 308 Evaporated

a 408 Evaporated

a 508 Evaporated

a 608 Evaporated

a 708 Evaporated

a 808 Evaporated

C 908 Evaporated

e 958 Evaporated

Final Boiling Point

Recovery

Regidue

Loss

Total Acid Number

\section{Description}

HTI PB-03-9, 10, 11 CRUDE OIL

180-350 Deg. F

Method

$\mathrm{D} 4052$

$\mathrm{D} 4052$

Wt. \&

Wt. $q$

Wt. \&

ppm

ppm

psi

Vol. o

Vol. \&

Vol. o

Vol. :

Vol. :

$\mathrm{mg} / 100 \mathrm{~mL}$

min.

Deg. F

Deg. F

Deg. F

Deg. F

Deg. F

Deg. F

Deg. F

Deg. F

Deg. F

Deg. F

Deg. F

Deg. F

Deg. F

Vol. 8

vol. 8

Vol. \&

$\mathrm{mgKOH} / \mathrm{g}$

D5291

D5291

D4294

D4629

UOP163

D 323

G.C.

G.C.

G.C.

G.C.

G.C.

D130

D381

D525

D2699

D2700

D86
004-00

43.7

0.8076

80.45

12.74

0.21

2005.0

669

1.7

22.15

5.01

55.26

17.58

72.84

$2 d$

50

$>240$

65.6

66.5

176

184

234

243

251

260

271

284

298

311

328

341

352

96.6

0.6

1.1

0.14 


\title{
Inchcape Testing Services
}

Caleb Brett

\author{
Laboratory Report No. 96-005170-0-HoUs; 1 - Page 5 of 8
}

Sample ID

96-005170-0-HOUS-005-00

Test

API Gravity e $60 / 60 \mathrm{~F}$ Specific Gravity @ $60 / 60 \mathrm{~F}$ Carbon

Hydrogen

sulfur Content

Total Nitrogen

Mercaptan Sulfur Content

Viscosity a $-20 \mathrm{C}$

Viscosity a $100 \mathrm{C}$

Freezing Point

Pour point

Flash Point, TCC

Vapor Pressure

Luminometer Number

Smoke Point

Paraffins

Olefins

Naphthenes

Aromatics

Total N\&A

Naphthalenes

Corrosion 3 hrs a 122 F

Existent Gum

Tube Rating

Pressure Drop

Oxidation stability

Research Octane Number

Motor Octane Number

Cetane Number

Initial Boiling point

a $5 \%$ Evaporated

e 108 Evaporated

e 20\% Evaporated

a 308 Evaporated

e 408 Evaporated

e 508 Evaporated

e 608 Evaporated

c 70\% Evaporated

a $80 \%$ Evaporated

e $90 \%$ Evaporated

e 95\% Evaporated

Final Boiling Point

Recovery

Residue

Loss

Net Heat of Combustion

Total Acid Number
Description

HTI PB-03-9, 10, 11 CRUDE OIL

350-400 Deg. F

\begin{tabular}{|c|c|c|c|}
\hline & & Method & $005-00$ \\
\hline & & $\begin{array}{l}\text { D } 4052 \\
\text { D4052 }\end{array}$ & $\begin{array}{r}21.2 \\
0.9268\end{array}$ \\
\hline Wt. $\%$ & & D5291 & 81.77 \\
\hline Wt. \& & & D5291 & 10.54 \\
\hline Wt. 8 & & D4294 & 0.17 \\
\hline ppm & & D4629 & 5897.0 \\
\hline $\mathrm{ppm}$ & & UOP163 & 597 \\
\hline cst & & D445 & 12.42 \\
\hline cst & & D445 & 0.750 \\
\hline Deg. & $F$ & D2386 & -56.0 \\
\hline Deg. & F & D97 & $<-76.0$ \\
\hline Deg. & F & D56 & 150 \\
\hline psi & & D323 & 0.2 \\
\hline & & D1740 & 33 \\
\hline $\mathrm{mm}$ & & D1322 & 13 \\
\hline Vol. & 8 & G.C. & 21.46 \\
\hline Vol. & 8 & G.C. & 9.20 \\
\hline Vol. & q & G.C. & 11.83 \\
\hline Vol. & 8 & G.C. & 57.51 \\
\hline Vol. & 8 & G.C. & 69.34 \\
\hline vol. & $z$ & D 1840 & 6.34 \\
\hline & & D130 & $3 a$ \\
\hline $\mathrm{mg} / 10$ & $0 \mathrm{~mL}$ & D381 & 63 \\
\hline & & D3241 & 4.0 \\
\hline $\mathrm{mmHg}$ & & D3241 & $>125.0$ \\
\hline min. & & D525 & $>240$ \\
\hline & & D2699 & 106.6 \\
\hline & & D2700 & 91.2 \\
\hline & & D613 & $<18.3$ \\
\hline $\begin{array}{l}\text { Deg. } \\
\text { Deg. }\end{array}$ & $\mathbf{F}$ & D86 & 351 \\
\hline $\begin{array}{l}\text { Deg. } \\
\text { Deg. }\end{array}$ & $\mathbf{F}$ & & 360 \\
\hline Deg. & $\mathbf{F}$ & & 361 \\
\hline Deg. & $\mathbf{F}$ & & 365 \\
\hline Deg. & $\mathbf{F}$ & & 367 \\
\hline Deg. & $F$ & & 369 \\
\hline Deg. & $F$ & & 370 \\
\hline Deg. & $F$ & & 372 \\
\hline Deg. & $F$ & & 376 \\
\hline Deg. & $F$ & & 378 \\
\hline Deg. & $F$ & & 383 \\
\hline Deg. & $F$ & & 388 \\
\hline Deg. & F & & 403 \\
\hline vol. & 8 & & 99.0 \\
\hline Vol. & & & 0.5 \\
\hline Vol. & & & 0.5 \\
\hline BTU / 1 & & D1405 & 18158 \\
\hline $\mathrm{mgKOH}$ & & D664 & 0.70 \\
\hline
\end{tabular}


Laboratory Report No. 96-005170-0-Hous; 1 - Page 6 of 8

Sample ID

96-005170-0-HOUS-006-00

Test

API Gravity @ 60/60 F

Specific Gravity e $60 / 60 \mathrm{~F}$

Carbon

Hydrogen

Sulfur Content

Total Nitrogen

Basic Nitrogen

Mercaptan Sulfur Content

viscosity a $-20 \mathrm{C}$

Viscosity a $40 \mathrm{C}$

viscosity a $100 \mathrm{C}$

Freezing point

Pour Point

Aniline Point

Flash Point, TCC

Luminometer Number

Smoke Point

Paraffins

olefins

Naphthenes

Aromatics

Total N\&A

Naphthalenes

Corrosion 3 hrs e $122 \mathrm{~F}$

Existent Gum

Tube Rating

Pressure Drop

Cetane Number

Initial Boiling point

a $5 \%$ Evaporated

a 108 Evaporated

a 208 Evaporated

c 308 Evaporated

e. 408 Evaporated

a 508 Evaporated

a 608 Evaporated

e 708 Evaporated

(a) 80\% Evaporated

a 908 Evaporated

c 958 Evaporated

Final Boiling Point

Recovery

Residue

Loss

Net Heat of Combustion

Total Acid Number

\section{Description}

HTI PB-03-9, 10, 11 CRUDE OIL

400-550 Deg. F $\underline{006-00}$

D4052 16.8

D4052 0.9540

D5291 83.85

D5291 $\quad 10.15$

D4294 0.12

D4629 8159.0

UOP269 5972

UOP163 126

D445 73.47

D445 4.216

D445 1.199

D2386 -19.0

D97 -33.0

D611 18.5

D56 >200

D1740 22

D1322

G.C.

G.C.

G.C.

G.C.

G.C.

D1840

D130

D381

D3241

D3241

mmHg

D613

Deg. F

Deg. F

D86

13.95

4.40

20.97

60.68

81.65

14.92

$1 a$

40

4.0

$<1.0$

$<18.3$

412

433

439

444

450

457

462

471

480

484

504

514

532

99.0

0.5

0.5

vol. :

Vol. \&

BTU/Ib

D1405

18190

D974 
Inchcape Testing Services

Caleb Brett

Laboratory Report No. 96-005170-0-HoUS; 1 - Page 7 of 8

Sample ID

96-005170-0-HOUS-007-00

Test

API Gravity e $60 / 60$ F

Specific Gravity a $60 / 60 \mathrm{~F}$

Carbon

Hydrogen

Sulfur Content

Total Nitrogen

Basic Nitrogen

Viscosity $40 \mathrm{C}$

Viscosity @ $100 \mathrm{C}$

Pour Point

Aniline Point

Flash Point (Method A)

Paraffins

Olefing

Naphthenes

Aromatics

Total N\&A

Bromine Number

Corrosion $3 \mathrm{hrs}$ a $122 \mathrm{~F}$

Cetane Number

Simulated Distillation
Description

HTI PB-03-9, 10, 11 CRUDE OIL

550-650 Deg. F

Method

$\underline{007-00}$

D 4052

D4052

Wt. $\%$

D5291

D5291

D4294

D4629

UOP2 69

D445

D445

ppm

cst

$\begin{array}{ll}\text { Deg. } F & \text { D97 } \\ \text { Deg. F } & \text { D611 }\end{array}$

Deg. F D93

Vol. \& G.C.

Vol. 8 G.C.

Vol. \& G.C.

Vol. \& G.C.

vol. \&

G.C.

D1159

D130

D613

D2887
12.9

0.9800

88.72

10.58

0.25

4547

11.12

7.456

5.0

48.0

$>200$

15.57

4.40

5.59

74.44

80.03

29

$1 a$

$<18.3$

See Attached
7197.0 
Laboratory Report No. 96-005170-0-Hous; 1 - Page 8 of 8

Sample ID

96-005170-0-HOUS-008-00

Test

API Gravity e 60/60 F Specific Gravity e $60 / 60 \mathrm{~F}$ Carbon Hydrogen

Sulfur Content

Total Nitrogen

Basic Nitrogen

Viscosity a $40 \mathrm{C}$

viscosity e $100 \mathrm{C}$

Pour Point

Aniline Point

Flash Point (Method A)

Microcarbon Residue

Corrosion 3 hrs e 122 F

Cetane Number

Initial Boiling Point

a $5 \%$ Recovery

(a) 10: Recovery

a $20 \%$ Recovery

e 30\% Recovery

(d) $40 \%$ Recovery

a 508 Recovery

a 608 Recovery

a 708 Recovery

(a) 80\% Recovery

e 908 Recovery

Final Boiling Point

Recovery

Residue + Loss
Description

HTI PB-03-9, 10, 11 CRUDE OIL

$650+$ Deg. F

\begin{tabular}{llr} 
& Method & O08-00 \\
& D4052 & \\
Wt. & D4052 & 6.4 \\
Wt. \& & D5291 & 1.0261 \\
Wt. \& & D5291 & 88.13 \\
Ppm & D4294 & 9.44 \\
Ppm & DO629 & 0.62 \\
CSt & D445 & 8584.0 \\
CSt & D445 & 4768 \\
Deg. F & D97 & 145.5 \\
Deg. F & D611 & 7.456 \\
Deg. F & D93 & 59.0 \\
Wt. 8 & D4530 & 113.0 \\
& D130 & $>200$ \\
Deg. F & D613 & $<0.1$ \\
Deg. F & D1160 & 12 \\
Deg. F & & N/A \\
Deg. F & & 656 \\
Deg. F & & 677 \\
Deg. F & & 678 \\
Deg. F & & 692 \\
Deg. F & & 700 \\
Deg. F & & 709 \\
Deg. F & & 724 \\
Deg. F & & 743 \\
Deg. F & & 773 \\
Vol. \& & & 871 \\
Vol. \& & & 880 \\
& & 880 \\
& & 84.0 \\
& & 16.0 \\
\hline
\end{tabular}




\section{Boiling Point Distribution}

\section{ASTM D-2887}

$\begin{array}{rrrrrr}\text { \%Off } & \text { BP(F) } & \text { BP(C) } & \text { \%Off } & \text { BP(F) } & \text { BP(C) } \\ \text { IBP } & 519.1 & 270.6 & \mathbf{4 0} & 603.5 & 317.5 \\ \mathbf{1} & 538.5 & 281.4 & \mathbf{4 1} & 604.5 & 318.1 \\ \mathbf{2} & 550.2 & 287.9 & \mathbf{4 2} & 605.6 & 318.7 \\ \mathbf{3} & 555.9 & 291.1 & \mathbf{4 3} & 606.6 & 319.2 \\ \mathbf{4} & 559.7 & 293.2 & \mathbf{4 4} & 607.7 & 319.8 \\ \mathbf{5} & 562.0 & 294.4 & \mathbf{4 5} & 608.7 & 320.4 \\ \mathbf{6} & 563.6 & 295.3 & \mathbf{4 6} & 609.8 & 321.0 \\ \mathbf{7} & 565.1 & 296.2 & \mathbf{4 7} & 610.8 & 321.6 \\ \mathbf{8} & 566.5 & 296.9 & \mathbf{4 8} & 611.9 & 322.2 \\ \mathbf{9} & 567.8 & 297.7 & \mathbf{4 9} & 612.9 & 322.7 \\ \mathbf{1 0} & 569.2 & 298.4 & \mathbf{5 0} & 614.0 & 323.3 \\ \mathbf{1 1} & 570.6 & 299.2 & \mathbf{5 1} & 615.0 & 323.9 \\ \mathbf{1 2} & 572.1 & 300.1 & \mathbf{5 2} & 616.1 & 324.5 \\ \mathbf{1 3} & 573.6 & 300.9 & \mathbf{5 3} & 617.2 & 325.1 \\ \mathbf{1 4} & 575.1 & 301.7 & \mathbf{5 4} & 618.3 & 325.7 \\ \mathbf{1 5} & 576.5 & 302.5 & \mathbf{5 5} & 619.4 & 326.3 \\ \mathbf{1 6} & 577.8 & 303.2 & \mathbf{5 6} & 620.5 & 326.9 \\ \mathbf{1 7} & 578.9 & 303.8 & \mathbf{5 7} & 621.5 & 327.5 \\ \mathbf{1 8} & 579.9 & 304.4 & \mathbf{5 8} & 622.6 & 328.1 \\ \mathbf{1 9} & 580.9 & 304.9 & \mathbf{5 9} & 623.6 & 328.7 \\ \mathbf{2 0} & 581.9 & 305.5 & \mathbf{6 0} & 624.7 & 329.3 \\ \mathbf{2 1} & 582.9 & 306.1 & \mathbf{6 1} & 625.7 & 329.8 \\ \mathbf{2 2} & 584.0 & 306.7 & \mathbf{6 2} & 626.8 & 330.4 \\ \mathbf{2 3} & 585.2 & 307.3 & \mathbf{6 3} & 628.0 & 331.1 \\ \mathbf{2 4} & 586.4 & 308.0 & \mathbf{6 4} & 629.1 & 331.7 \\ \mathbf{2 5} & 587.7 & 308.7 & \mathbf{6 5} & 630.2 & 332.3 \\ \mathbf{2 6} & 588.9 & 309.4 & \mathbf{6 6} & 631.3 & 332.9 \\ \mathbf{2 7} & 590.1 & 310.1 & \mathbf{6 7} & 632.3 & 333.5 \\ \mathbf{2 8} & 591.2 & 310.7 & \mathbf{6 8} & 633.4 & 334.1 \\ \mathbf{2 9} & 592.3 & 311.3 & \mathbf{6 9} & 634.5 & 334.7 \\ \mathbf{3 0} & 593.4 & 311.9 & \mathbf{7 0} & 635.8 & 335.4 \\ \mathbf{3 1} & 594.4 & 312.4 & \mathbf{7 1} & 637.1 & 336.2 \\ \mathbf{3 2} & 595.4 & 313.0 & \mathbf{7 2} & 638.5 & 336.9 \\ \mathbf{3 3} & 596.4 & 313.6 & \mathbf{7 3} & 639.9 & 337.7 \\ \mathbf{3 4} & 597.4 & 314.1 & \mathbf{7 4} & 641.2 & 338.4 \\ \mathbf{3 5} & 598.4 & 314.7 & \mathbf{7 5} & 642.4 & 339.1 \\ \mathbf{3 6} & 599.4 & 315.2 & \mathbf{7 6} & 643.6 & 339.8 \\ \mathbf{3 7} & 600.4 & 315.8 & \mathbf{7 7} & 644.8 & 340.4 \\ \mathbf{3 8} & 601.4 & 316.3 & \mathbf{7 8} & 646.0 & 341.1 \\ \mathbf{3 9} & 602.4 & \mathbf{3 1 6 . 9} & \mathbf{7 9} & 647.2 & 341.8\end{array}$

\begin{tabular}{|c|c|c|}
\hline$\%$ Off & $\mathrm{BP}(\mathrm{F})$ & $\mathrm{BP}(\mathrm{C})$ \\
\hline 80 & 648.5 & 342.5 \\
\hline 81 & 649.8 & 343.2 \\
\hline 82 & 651.1 & 343.9 \\
\hline 83 & 652.2 & 344.6 \\
\hline 84 & 653.3 & 345.2 \\
\hline 85 & 654.3 & 345.7 \\
\hline 86 & 655.4 & 346.3 \\
\hline 87 & 656.4 & 346.9 \\
\hline 88 & 657.4 & 347.4 \\
\hline 89 & 658.7 & 348.2 \\
\hline 90 & 660.2 & 349.0 \\
\hline 91 & 661.9 & 349.9 \\
\hline 92 & 663.6 & 350.9 \\
\hline 93 & 665.5 & 351.9 \\
\hline 94 & 667.8 & 353.2 \\
\hline 95 & 669.9 & 354.4 \\
\hline 96 & 672.3 & 355.7 \\
\hline 97 & 675.6 & 357.6 \\
\hline 98 & 677.6 & 358.7 \\
\hline 99 & 685.4 & 363.0 \\
\hline FBP & 8 & 372.1 \\
\hline
\end{tabular}

Start Time: 0.7 minutes

End Time: 17.8 minutes

Area: 272058272.0

Stice Width: $0.80 \mathrm{sec}$

Baseline Subtracted: c:ltc4lsd689011111
Sample Offset: 8638.0

Baseline Offset: 8792.0

Calibration File: $1111 \mathrm{rt}$

Calibration Date: $11 / 11 / 96$ 


\section{Boiling Point Distribution}

\section{ASTM D-2887}

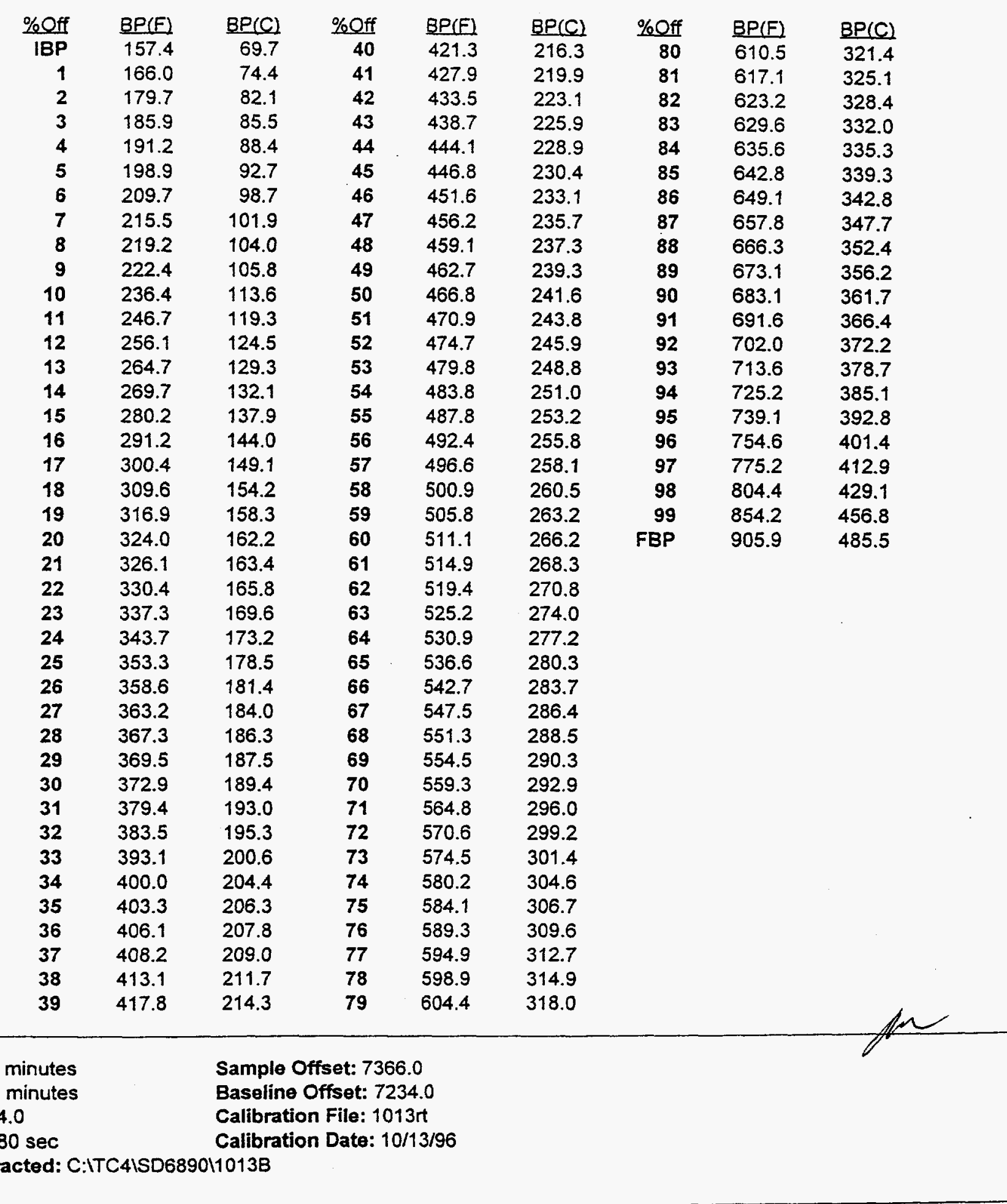

Start Time: 0.2 minutes

End Time: 26.0 minutes

Area: 39163064.0

Slice Width: $0.80 \mathrm{sec}$

Baseline Subtracted: C:ITC4ISD689011013B 
Inchcape Testing Services Caleb Brett

WinAssay ${ }^{\prime} 95$

Version 1.00

Final Reports

\begin{tabular}{|ll|}
\hline Client Name: & Consol Inc. \\
Sample ID: & $\underline{\text { HTI PB-03-9,10,11 (Charge \#1) }}$ \\
Laboratory ID: & $\underline{96-005170}$ \\
Date: & $\underline{10 / 01 / 96}$ \\
Operator: & $\underline{\text { Robert Kelly }}$ \\
\hline
\end{tabular}




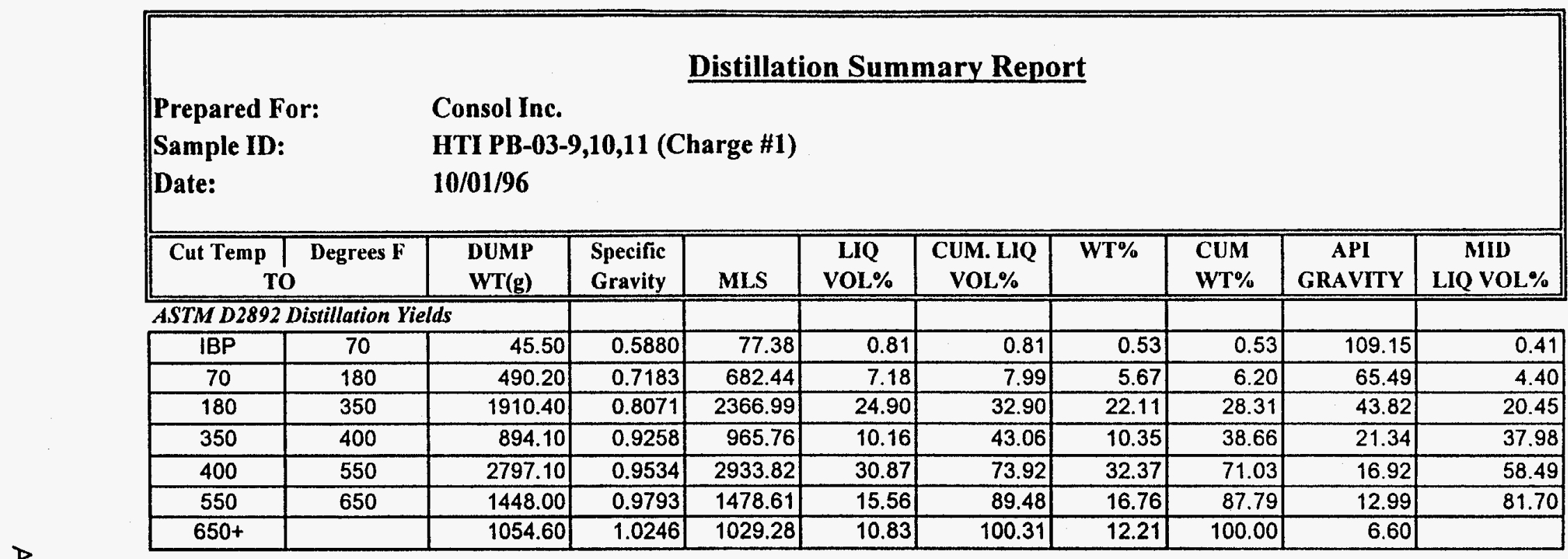

Loss (Grams): 9.2 (0.11 Wt.\%)

Distribution: (2/3) $6.1 \mathrm{~g}$ to IBP-70 F

(1/3) $3.1 \mathrm{~g}$ to $70-180 \mathrm{~F}$ 
WinAssay '95 Quality Control Applications

Cum. Mid Vol\% v. API Gravity

HTI PB-03-9,10,11 (Charge \#1)

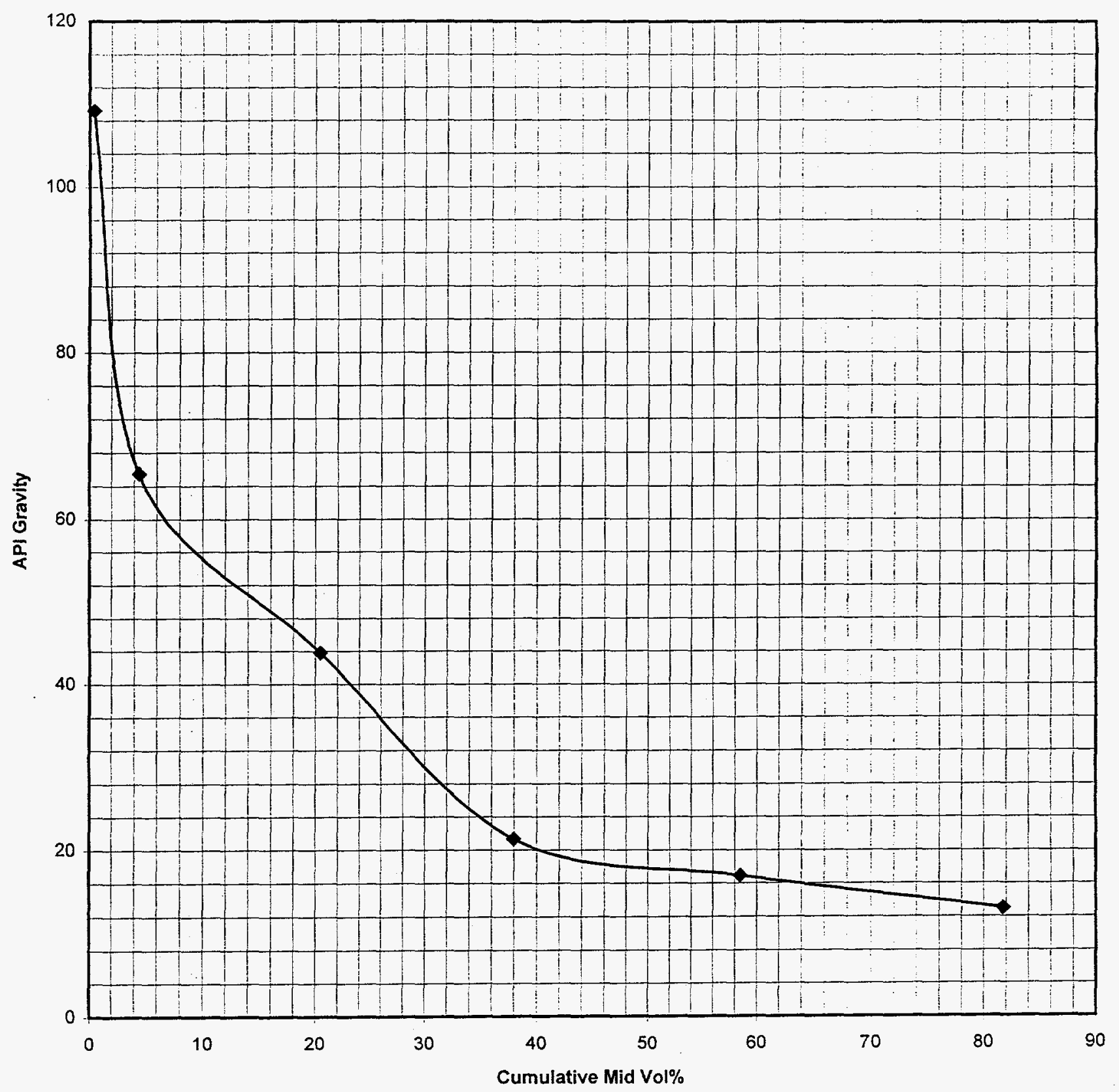




\section{WinAssay ' 95 \\ True Boiling Point Curve \\ Vaporline Temperature v. Cumulative $\mathrm{Wt} \%$ Yield}

Sample ID

HTI PB-03-9,10,11 (Charge \#1)

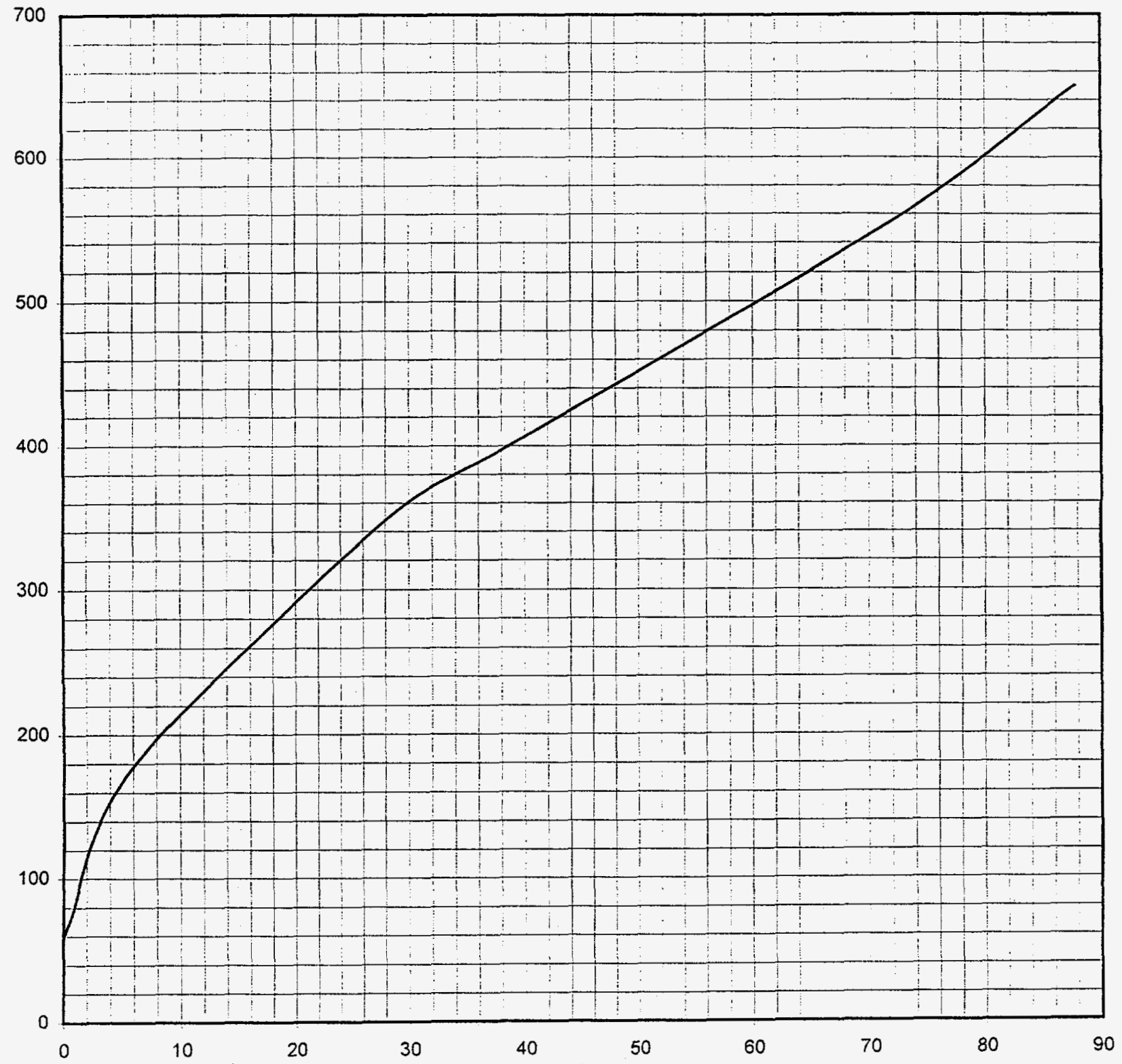




\section{WinAssay ' 95 \\ True Boiling Point Curve vs Cumulative Vol\% Yield}

Sample ID

HTI PB-03-9,10,11 (Charge \#1)

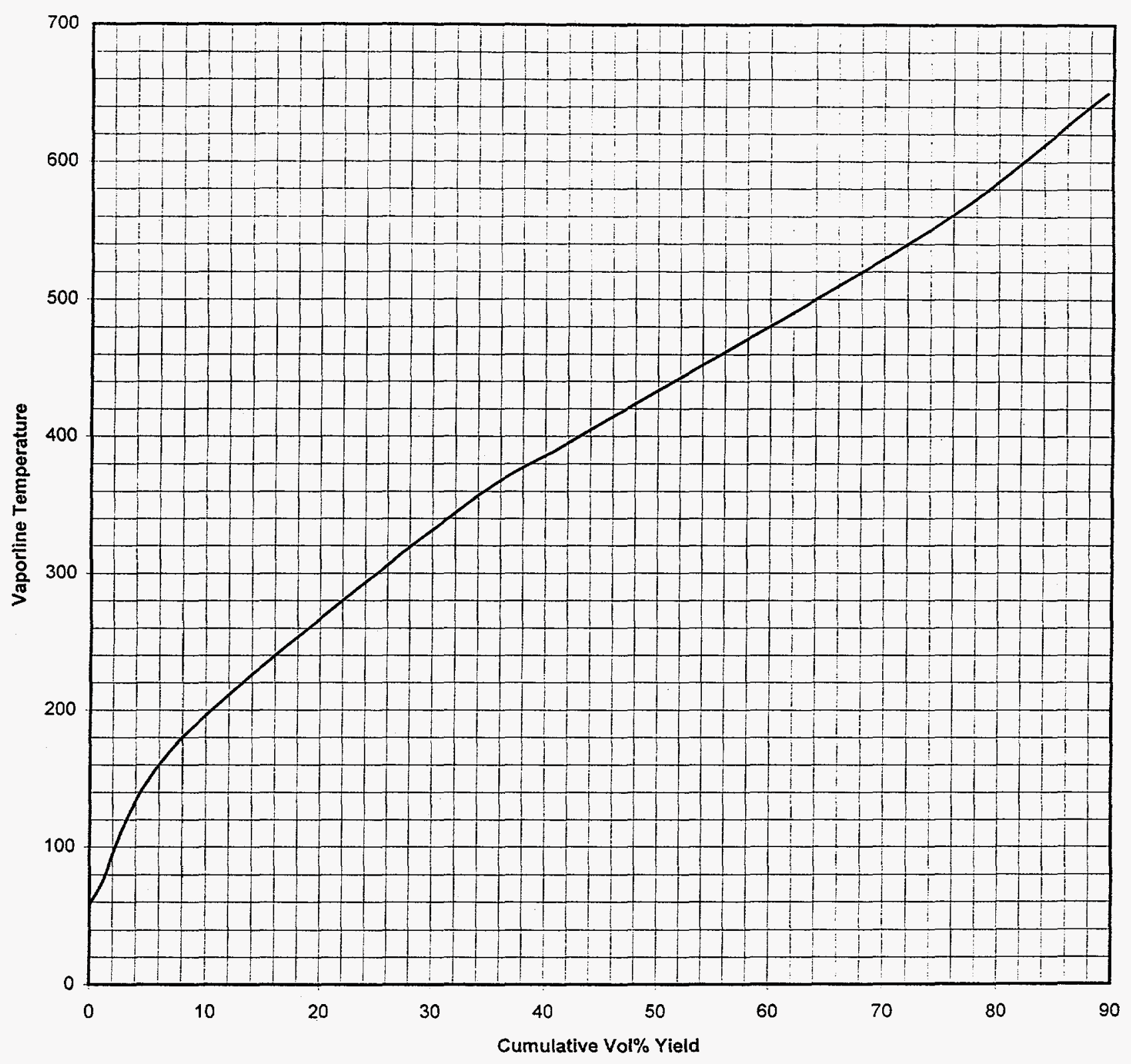


ITS- Caleb Brett Distillation Group

ASTM D2892/D5236 CHARGE INFORMATION

Lab ID:

Client Name:

Sample ID:

Date:

Charge Mass D2892(g):

Charge S.G D2892 (60/60F):

Charge Mass D5236(g):

Charge S.G. D5236 (60/60F):
96-005170

Consol Inc.

HTI PB-03-9,10,11 (Charge \#1)

$10 / 01 / 96$

离

$\begin{array}{r}\hline 8702.0 \\ \hline 0.9090 \\ \hline\end{array}$

0.0
Operator: Robert Kelly

Water Weight Removed (g):

Initial Vapor Temp:

Whole Crude Sulfur Wt\%:

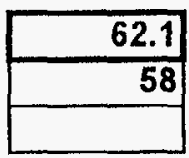

0.0000 
LALEB BEEI"I' HUU'S'LUN

\section{4}

'ILD: $90 \dot{0}-005170-U-H O U S-U U Z-U U$

CID: CONSOLINC

3ID: HTI PB-03-9,10,11 CKUDE OIL/IBP-70 E

NID: 51870 Date: 16-SEP-1996
Analyzed: $10 / 2 / 96 \quad 8: 25$ AM

Reported: 10-03-1996 13:00:05 Normalized to $100.00 \%$

Composite Report
Totals by Group Type \& Carbon Number
(in Weight Percent)

Paraffins: I-paraffins: Aromatics: Naphthenes: Olefins: Total:

$\begin{array}{lrrrrrr}\text { C1: } & 0.000 & 0.000 & 0.000 & 0.000 & 0.000 & 0.000 \\ \text { C2: } & 0.009 & 0.000 & 0.000 & 0.000 & 0.000 & 0.009 \\ \text { C3: } & 2.051 & 0.000 & 0.000 & 0.000 & 0.052 & 2.103 \\ \text { C4: } & 62.570 & 7.306 & 0.000 & 0.000 & 5.875 & 75.751 \\ \text { C5: } & 8.407 & 7.611 & 0.000 & 1.239 & 1.541 & 18.797 \\ \text { C6: } & 0.727 & 0.837 & 0.041 & 1.101 & 0.207 & 2.914 \\ \text { C7: } & 0.021 & 0.037 & 0.000 & 0.110 & 0.027 & 0.196 \\ \text { C8: } & 0.000 & 0.000 & 0.000 & 0.000 & 0.000 & 0.000 \\ \text { C9: } & 0.000 & 0.000 & 0.000 & 0.000 & 0.000 & 0.000 \\ \text { C10: } & 0.000 & 0.000 & 0.000 & 0.000 & 0.000 & 0.000 \\ \text { C11: } & 0.000 & 0.000 & 0.000 & 0.000 & 0.000 & 0.000 \\ \text { C12: } & 0.000 & 0.000 & 0.000 & 0.000 & 0.000 & 0.000 \\ \text { C13: } & 0.000 & 0.000 & 0.000 & 0.000 & 0.000 & 0.000 \\ \text { C14: } & 0.000 & 0.000 & 0.000 & 0.000 & 0.000 & 0.000 \\ \text { Total: } 73.784 & 15.791 & 0.041 & 2.450 & 7.702 & 99.769 \\ & & & & & & \text { Total Unknowns: } \\ \text { Oxygenates: } & 0.017 & \text { Total C14+: } & 0.000 & & 0.214 \\ & & & & & \text { Grand Total: I00.000 }\end{array}$

Molecular Weight and Relative Density Data

$\begin{array}{crc}\text { Group: } & \text { Ave. Mw.: } & \text { Ave. Rel. Density: } \\ \text { CI: } & 0.000 & 0.000 \\ \text { C2: } & 30.070 & 0.340 \\ \text { C3: } & 44.045 & 0.501 \\ \text { C4: } & 57.962 & 0.579 \\ \text { C5: } & 71.833 & 0.632 \\ \text { C6: } & 85.077 & 0.700 \\ \text { C7: } & 98.782 & 0.731 \\ \text { C8: } & 0.000 & 0.000 \\ \text { C9: } & 0.000 & 0.000 \\ \text { C10: } & 0.000 & 0.000 \\ \text { C11: } & 0.000 & 0.000 \\ \text { C12: } & 0.000 & 0.000 \\ \text { C13: } & 0.000 & 0.000 \\ \text { C14: } & 0.000 & 0.000 \\ \text { Total Sample: } & 60.242 & 0.588\end{array}$

File: 5170 S1. DHA 
LALEB BKEI"I' HUUSTUN

IID: Sல-UO517U-U-HOUS-UUŻ-UU

CID: CONSOLINC'

SID: HTI PB-U3-9,10,11 CRULE OIL/IBP-7U E

NID: 51870

Late: $16-S E P-1996$

Analyzed: 10/2/96 8:25 AM

Reported: 10-03-1996 13:00:05

Normalized to $100.00 \%$

Composite Report

Totals by Group Type \& Carbon Number

(in Volume Percent)

Paraffins

$\begin{array}{lr}\text { C1: } & 0.000 \\ \text { C2: } & 0.015 \\ \text { C3: } & 2.416 \\ \text { C4: } & 63.715 \\ \text { C5: } & 7.912 \\ \text { C6: } & 0.650 \\ \text { C7: } & 0.018 \\ \text { C8: } & 0.000 \\ \text { C9: } & 0.000 \\ \text { C10: } & 0.000 \\ \text { C11: } & 0.000 \\ \text { C12: } & 0.000 \\ \text { C13: } & 0.000 \\ \text { C14: } & 0.000 \\ \text { C14 } & 74.726\end{array}$

Total: 74.726
I-paraffins

0.000

0.000

0.000

7.728

7.242

0.751

0.032

0.000

0.000

0.000

0.000

0.000

0.000

0.000

15.753
Aromatics

0.000

0.000

0.000

0.000

0.000

0.028

0.000

0.000

0.000

0.000

0.000

0.000

0.000

0.000

0.028
Olefins:

0.000

0.000

0.060

5.721

1. 389

0.173

0.022

0.000

0.000

0.000

0.000

0.000

0.000

0.000

7.365
Total:

0.000

0.015

2.476

77.164

17.523

2.454

0.158

0.000

0.000

0.000

0.000

0.000

0.000

0.000

99.789

Oxygenates: 0.012 Total C14t: 0.000

Total Unknowns:

0.199

Grand Total: 100.000

(in Mole Percent)

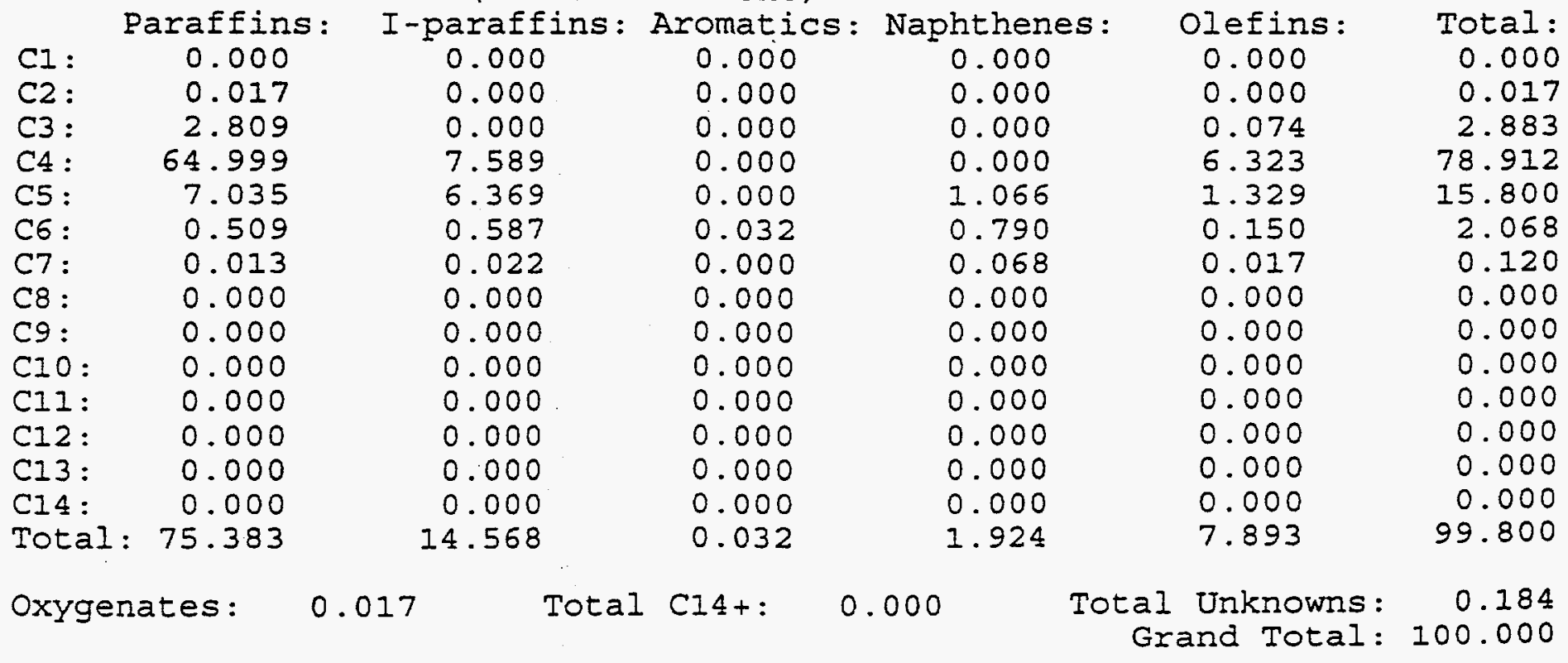


CALEB BREI"I' HUUS'IUH

TID: 9ல-UO5 $170-U-H O U S-U U 2-U U$

C.ID: CONSOLINC

GID: HTI YB-O3-9,10,11 CKULE $O I L / I B E-70$ F

NIU: 51870

Date: $16-S E P-1996$

Analyzed: $10 / 2 / 96$ 8:25 AM

Reported: 10-03-1996 13:00:05 Normalized to $100.00 \%$

Boiling Point Distribution Data

$\begin{array}{rrrrr}\text { IBP }(0.5 \%) & -42.04 & -43.67 & -42.04 & -43.67 \\ 5.0 \% & -11.72 & 10.90 & -11.72 & 10.90 \\ 10.0 \% & -6.25 & 20.75 & -11.72 & 10.90 \\ 15.0 \% & -0.50 & 31.10 & -0.50 & 31.10 \\ 20.0 \% & -0.50 & 31.10 & -0.50 & 31.10 \\ 25.0 \% & -0.50 & 31.10 & -0.50 & 31.10 \\ 30.0 \% & -0.50 & 31.10 & -0.50 & 31.10 \\ 35.0 \% & -0.50 & 31.10 & -0.50 & 31.10 \\ 40.0 \% & -0.50 & 31.10 & -0.50 & 31.10 \\ 45.0 \% & -0.50 & 31.10 & -0.50 & 31.10 \\ 50.0 \% & -0.50 & 31.10 & -0.50 & 31.10 \\ 55.0 \% & -0.50 & 31.10 & -0.50 & 31.10 \\ 60.0 \% & -0.50 & 31.10 & -0.50 & 31.10 \\ 65.0 \% & -0.50 & 31.10 & -0.50 & 31.10 \\ 70.0 \% & -0.50 & 31.10 & -0.50 & 31.10 \\ 75.0 \% & 0.88 & 33.58 & -0.50 & 31.10 \\ 80.0 \% & 27.84 & 82.11 & 20.05 & 68.09 \\ 85.0 \% & 27.84 & 82.11 & 27.84 & 82.11 \\ 90.0 \% & 36.06 & 96.91 & 36.06 & 96.91 \\ 95.0 \% & 36.34 & 97.41 & 36.06 & 96.91 \\ \text { FBP }(99.5 \%) & 80.72 & 177.30 & 80.72 & 177.30\end{array}$

Research Octane Number $=106.27$ (Calculated from Individual Component Values)

Contribution to Total by:

$\begin{array}{lr}\text { Paraffins: } & 78.57 \\ \text { Iso-paraffins: } & 17.09 \\ \text { Aromatics: } & 0.04 \\ \text { Naphthenes: } & 2.14 \\ \text { Olefins: } & 8.20 \\ \text { Oxygenates: } & 0.02\end{array}$

File: 5170s1.DHA 
LALEB BEEI"I HUU'O'lUN

\section{4}

'L10: $90-U 0517 U-U-H O U S-U U 2-U O$

CIID: GUNSOLINC

जID: HTL YB-03-9,10,11 CKUDE $O[L / I B E-7 \cup \mathrm{E}$

NIU: $518 T^{\circ}$ Date: $16-S E P-1996$

Analyzed: 10/2/96 8:25 AM

Reported: 10-03-1996 13:00:05

Normalized to $100.00 \%$

Components Listed in Chromatographic order

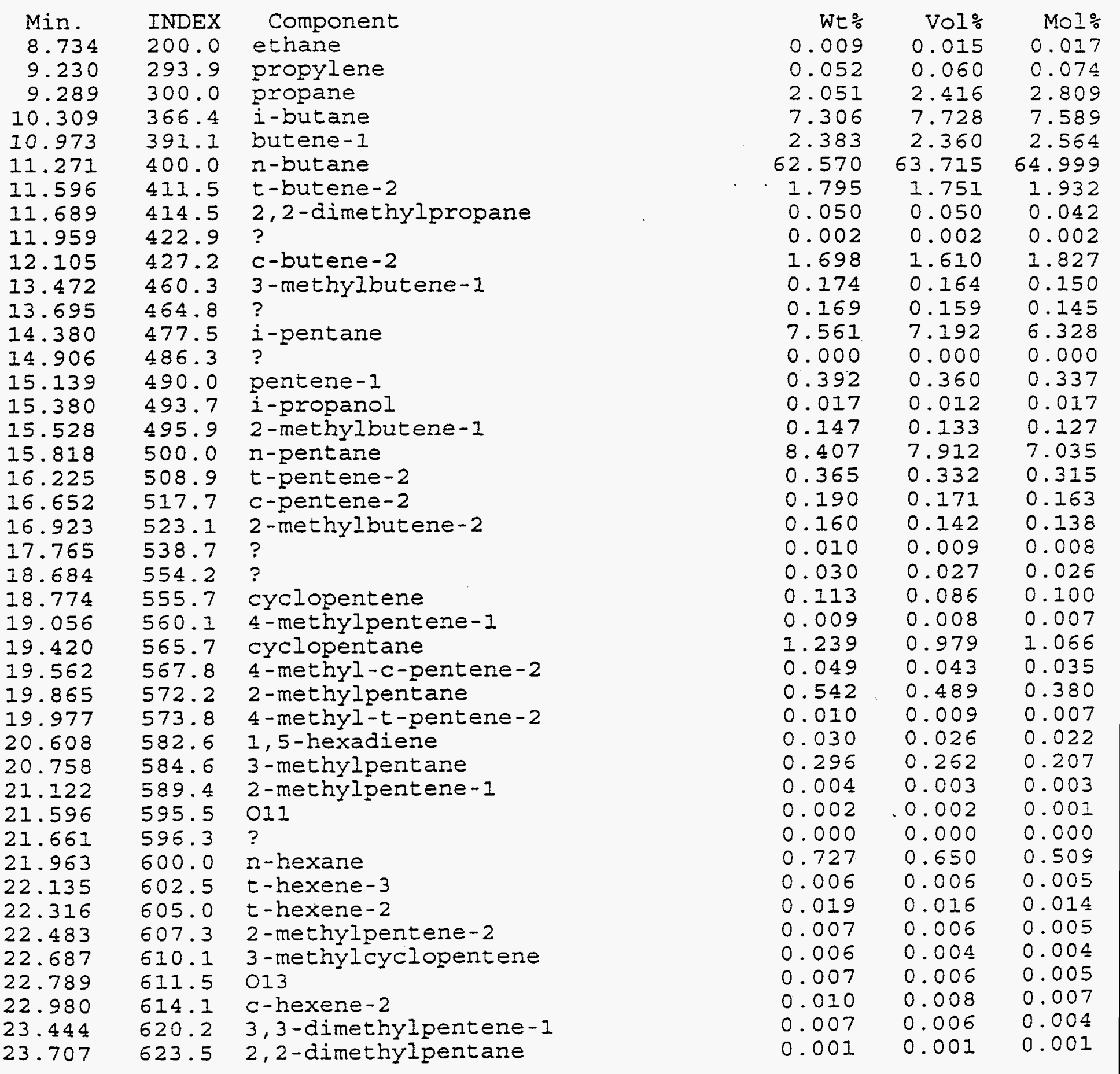

File: 5170S1.DHA Sample: 96-5170-s1 p. 1 
Components Listed in Chromatographic Order

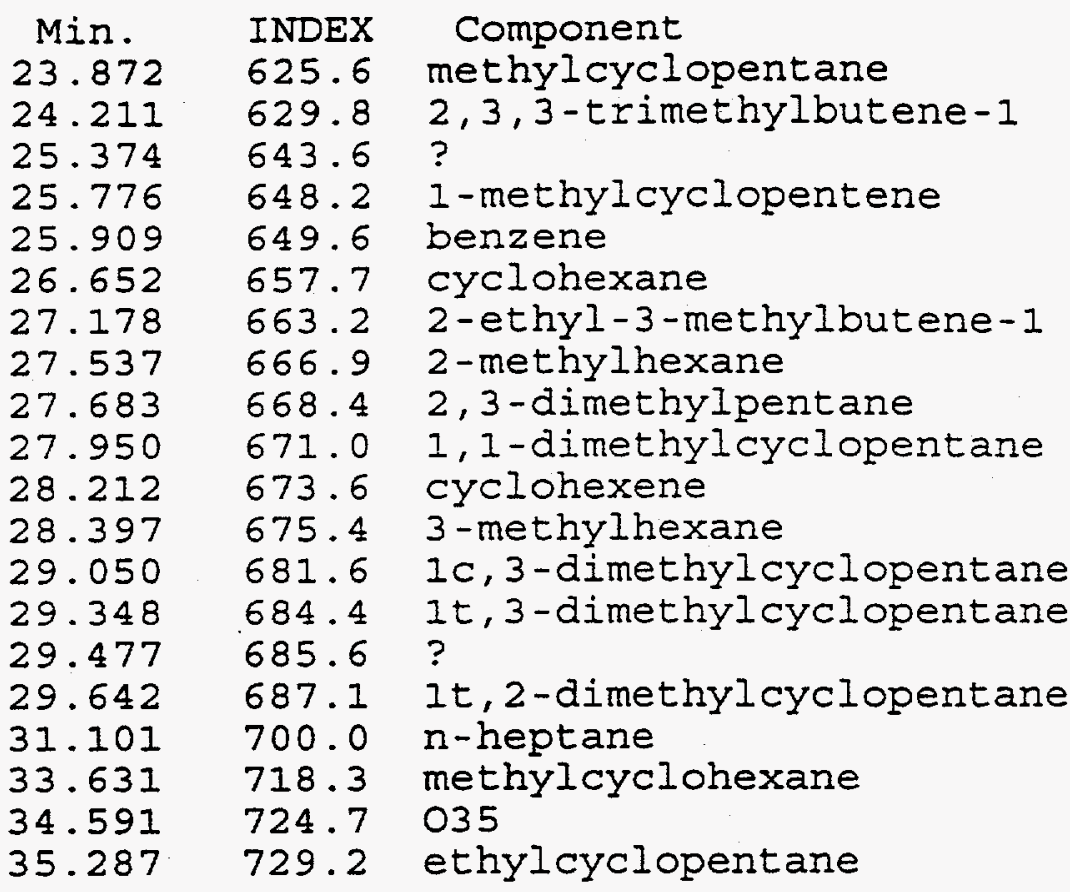

$\begin{array}{lll}\text { Wt } & \text { VOI\% } & \text { Mol\% } \\ 0.620 & 0.488 & 0.445 \\ 0.005 & 0.004 & 0.003 \\ 0.002 & 0.001 & 0.001 \\ 0.016 & 0.012 & 0.012 \\ 0.041 & 0.028 & 0.032 \\ 0.482 & 0.365 & 0.346 \\ 0.001 & 0.001 & 0.000 \\ 0.015 & 0.013 & 0.009 \\ 0.005 & 0.004 & 0.003 \\ 0.003 & 0.002 & 0.002 \\ 0.032 & 0.023 & 0.023 \\ 0.016 & 0.014 & 0.010 \\ 0.012 & 0.010 & 0.007 \\ 0.010 & 0.008 & 0.006 \\ 0.002 & 0.001 & 0.001 \\ 0.018 & 0.014 & 0.011 \\ 0.021 & 0.018 & 0.013 \\ 0.059 & 0.045 & 0.036 \\ 0.015 & 0.012 & 0.009 \\ 0.008 & 0.006 & 0.005\end{array}$

File: 5170S1.DHA. Sample: 96-5170-sI

p. 2 


\section{Inchcape Testing Services}

\# Caleb Brett

WinAssay '95

Version 1.00

Final Reports

\begin{tabular}{|ll||}
\hline Client Name: & $\underline{\text { Consol Inc. }}$ \\
Sample ID: & $\underline{\text { HTI PB-03-9,10,11 (Charge \#2) }}$ \\
Laboratory ID: & $\underline{96-005170}$ \\
Date: & $\underline{10 / 01 / 96}$ \\
Operator: & $\underline{\text { Robert Kelly }}$ \\
\hline
\end{tabular}




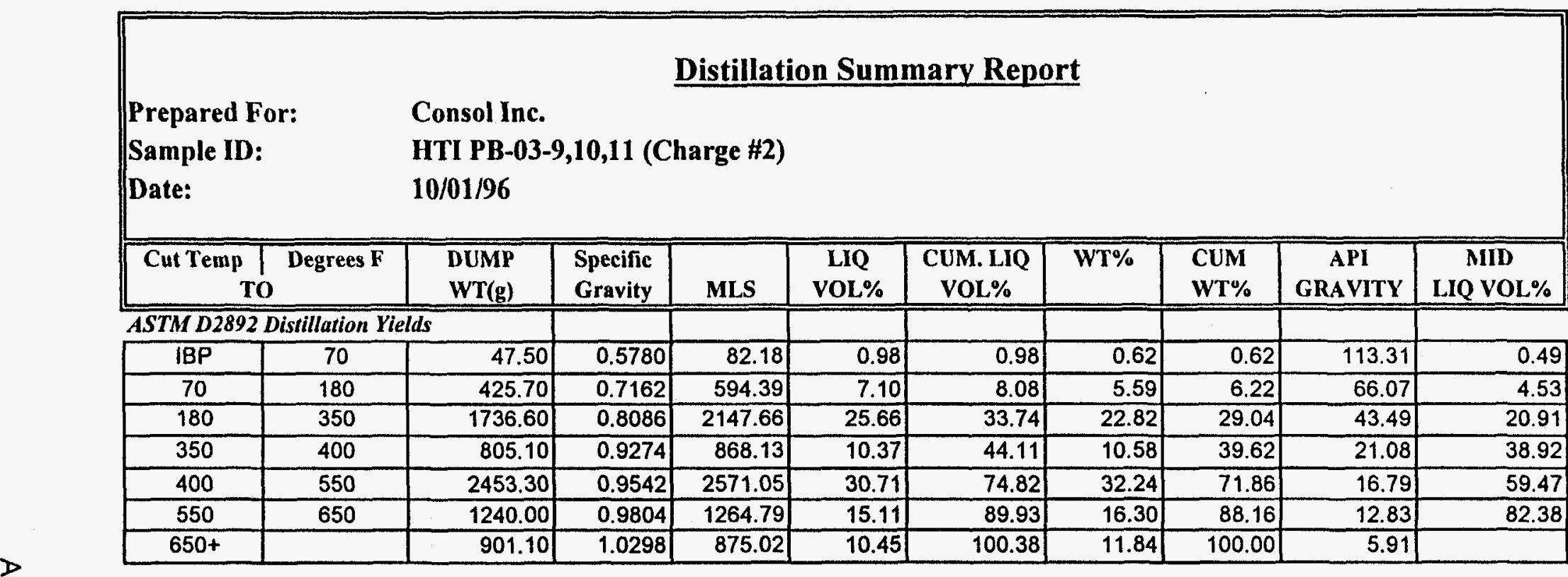

Loss (Grams): 16.2 (0.21 Wt.\%)

Distribution: (2/3) $10.8 \mathrm{~g}$ to IBP-70 F

(1/3) $5.4 \mathrm{~g}$ to $70-180 \mathrm{~F}$ 
WinAssay '95 Quality Control Applications

Cum. Mid Vol\% v. API Gravity

HTI PB-03-9,10,11 (Charge \#2)

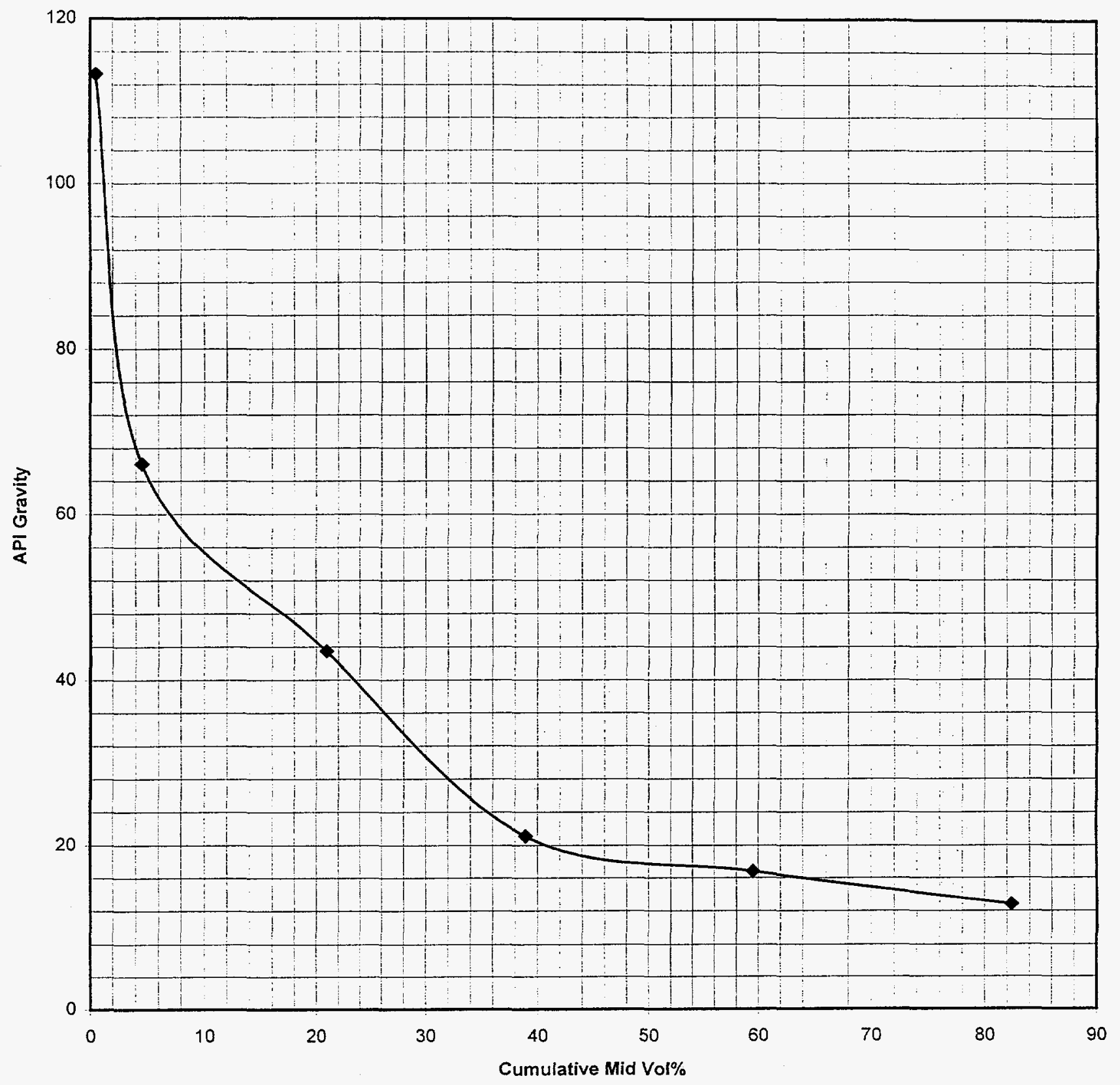




\section{WinAssay '95 \\ True Boiling Point Curve \\ Vaporline Temperature v. Cumulative Wt\% Yield}

Sample ID

HTI PB-03-9,10,11 (Charge \#2)

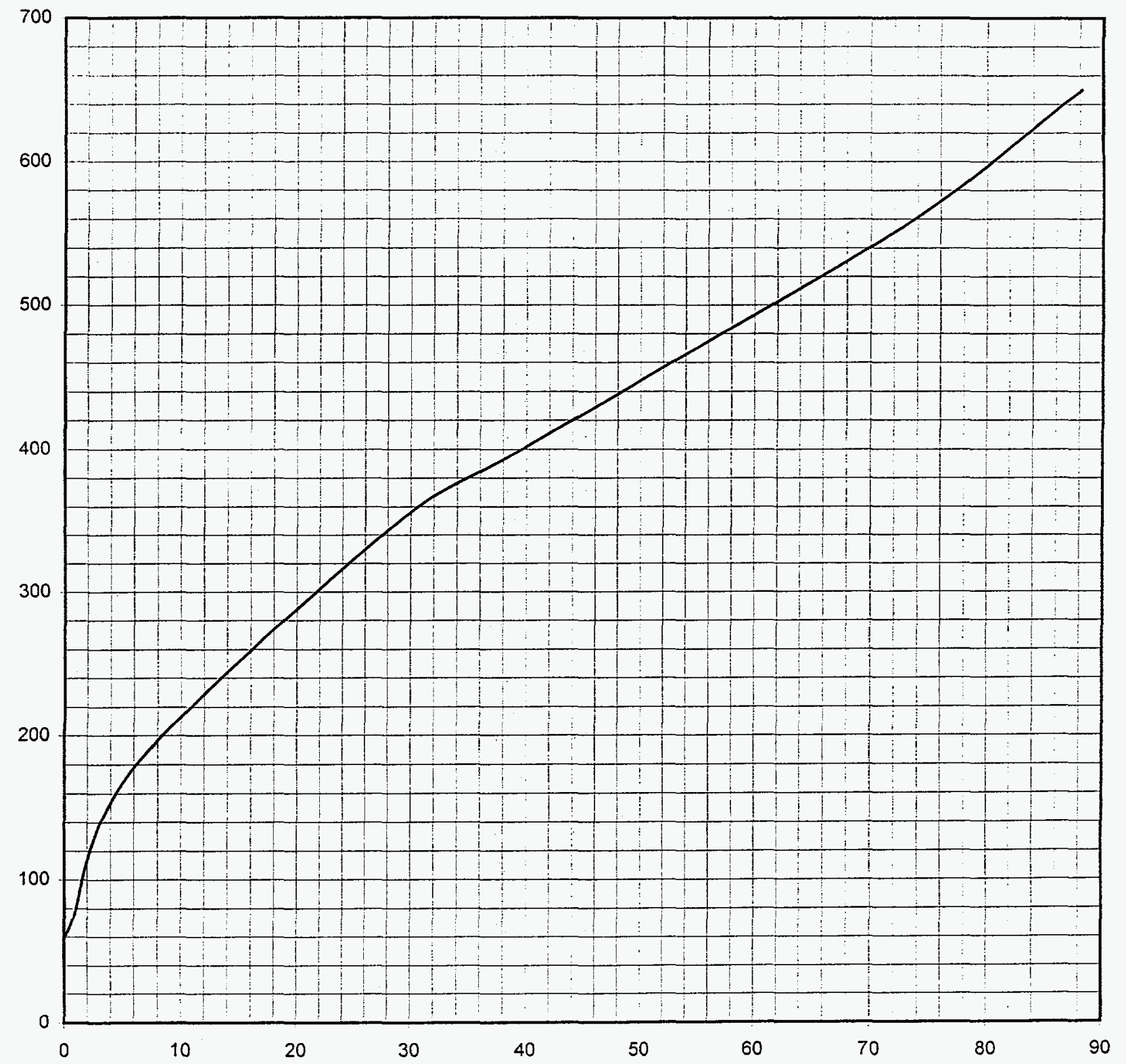




\section{WinAssay ' 95 \\ True Boiling Point Curve vs Cumulative Vol\% Yield}

Sample ID

HTI PB-03-9,10,11 (Charge \#2)

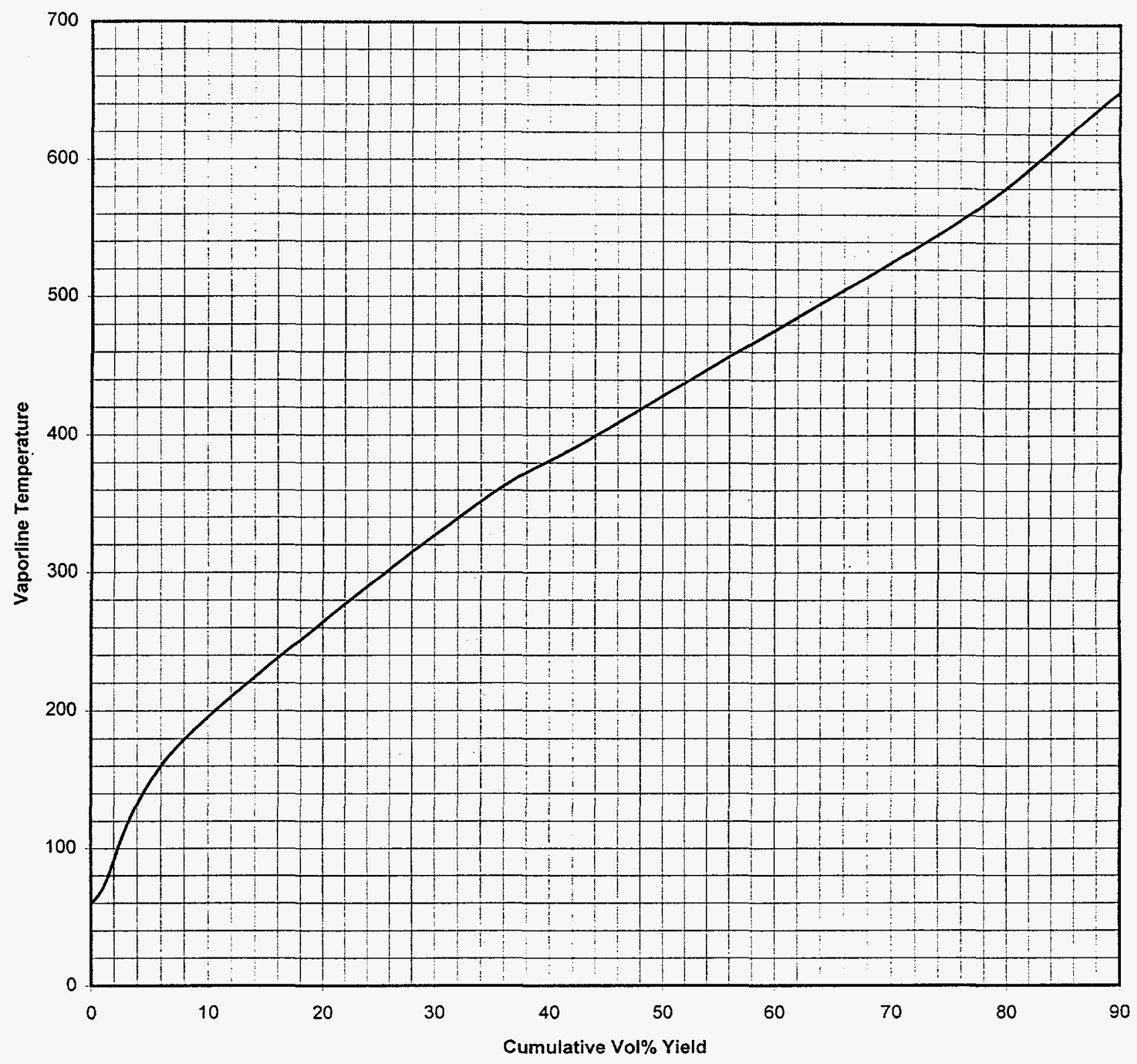




\section{ITS- Caleb Brett Distillation Group}

ASTM D2892/D5236 CHARGE INFORMATION

Lal) ID:

Client Name:

Sample ID:

96-005170

Consol Inc.

Date:

HTI PB-03-9,10,11 (Charge \#2)

$10 / 01 / 96$

Operator: Robert Kelly

Charge Mass D2892(g):

Charge S.G D2892 (60/60F):

\begin{tabular}{r|}
\hline 7669.0 \\
\hline 0.9090 \\
\hline
\end{tabular}

0.0

Charge Mass D5236(g):

Charge S.G. D5236 (60/60F):

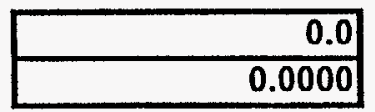

Water Weight Removed (g):

Initial Vapor Temp:

Whole Crude Sulfur Wt\%:

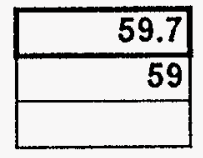

0.0000 
CALEB BKEI"I' HUU'S'IUW

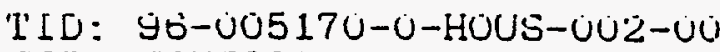

CID: CONSOLINC

BID: HTI PB-O3-9,10,11 CRULE OIL/IBP-7U E

NIU: 51870

Date: 16-SEP-1996

Analyzed: $10 / 1 / 963: 56 \mathrm{PM}$

Reported: 10-03-1996 13:02:33 Normalized to $100.00 \%$

Composite Report

Totals by Group Type \& Carbon Number (in Weight Percent)

Paraffins: I-paraffins: Aromatics: Naphthenes: Olefins: Total:

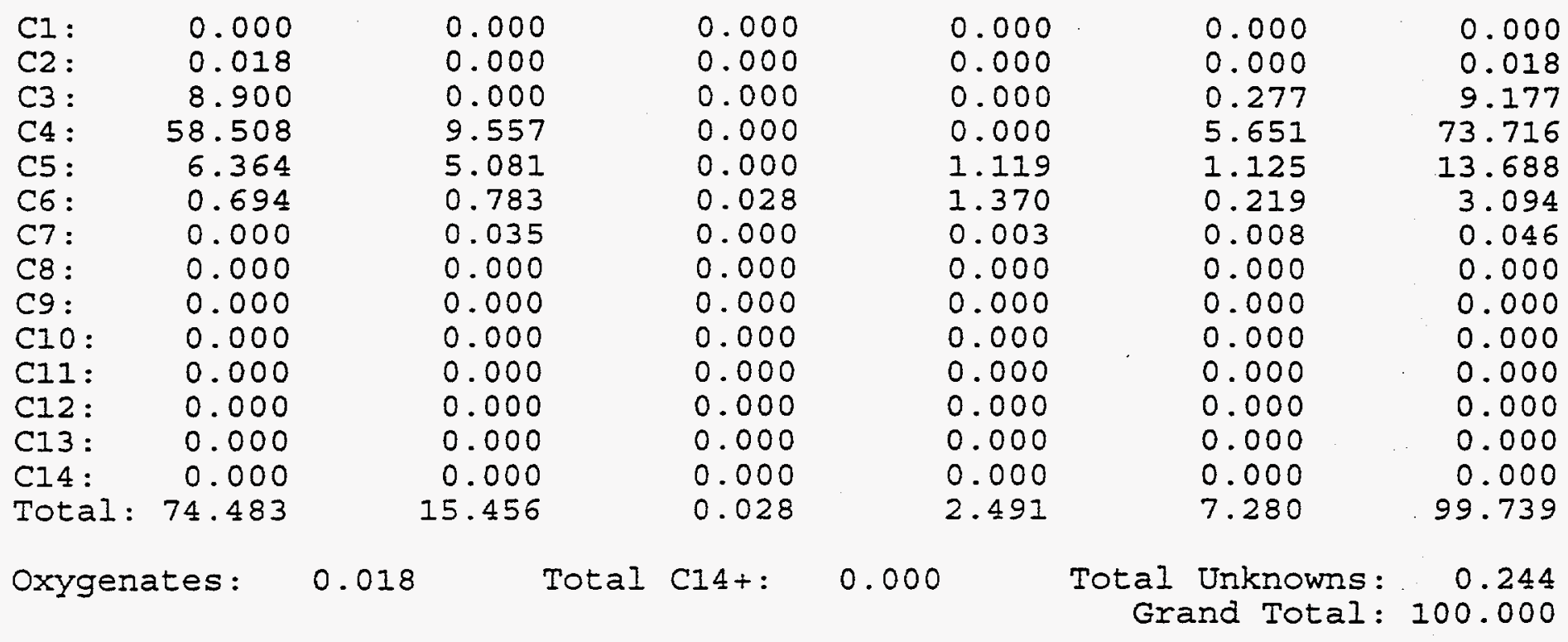

Molecular Weight and Relative Density Data

$\begin{array}{crr}\text { Group: } & \text { Ave. Mw.: } & \text { Ave. Rel. Density: } \\ \text { C1: } & 0.000 & 0.000 \\ \text { C2: } & 30.070 & 0.340 \\ \text { C3: } & 44.033 & 0.501 \\ \text { C4: } & 57.964 & 0.578 \\ \text { C5: } & 71.796 & 0.634 \\ \text { C6: } & 85.025 & 0.705 \\ \text { C7: } & 99.725 & 0.689 \\ \text { C8: } & 0.000 & 0.000 \\ \text { C9: } & 0.000 & 0.000 \\ \text { C10: } & 0.000 & 0.000 \\ \text { C11: } & 0.000 & 0.000 \\ \text { C12: } & 0.000 & 0.000 \\ \text { C13: } & 0.000 & 0.000 \\ \text { C14: } & 0.000 & 0.000 \\ \text { Sample: } & 58.258 & 0.578\end{array}$


LALEB BREI"I' HUUS'TUN

IID: $9 \dot{-0 O 5170-U-H O U S-U U \angle ்-U O ~}$

C.ID: CUNSOLINC'

BID: HTI PB-O3-9,10,11 CRULE OIL/IBP-7O E

NID: $51870^{\circ}$

Date: 16-SEP-1996

Analyzed: $10 / 1 / 963: 56 \mathrm{PM}$

Reported: $10-03-1996 \quad 13: 02: 33$

Normalized to $100.00 \%$

Composite Report

Totals by Group Type \& Carbon Number

(in Volume Percent)

\begin{tabular}{|c|c|c|c|c|c|c|}
\hline & Paraffins: & I-paraffins: & Aromatics: & Naphthenes: & Olefins: & Total \\
\hline C1: & 0.000 & 0.000 & 0.000 & 0.000 & 0.000 & 0.00 \\
\hline C2: & 0.030 & 0.000 & 0.000 & 0.000 & 0.000 & 0.03 \\
\hline C3: & 10.313 & 0.000 & 0.000 & 0.000 & 0.318 & 10 \\
\hline C4: & 58.626 & 9.947 & 0.000 & 0.000 & 5.428 & 74 \\
\hline C5: & 5.894 & 4.758 & 0.000 & 0.870 & 0.993 & 12.5 \\
\hline C6: & 0.611 & 0.691 & 0.019 & 1.041 & 0.186 & 2 \\
\hline C7: & 0.000 & 0.030 & 0.000 & 0.002 & 0.006 & 0 \\
\hline C8: & 0.000 & 0.000 & 0.000 & 0.000 & 0.000 & 0.00 \\
\hline C9: & 0.000 & 0.000 & 0.000 & 0.000 & 0.000 & 0.00 \\
\hline C10: & 0.000 & 0.000 & 0.000 & 0.000 & 0.000 & 0.00 \\
\hline C11: & 0.000 & 0.000 & 0.000 & 0.000 & 0.000 & 0.0 \\
\hline C12: & 0.000 & 0.000 & 0.000 & 0.000 & 0.000 & 0.00 \\
\hline C13: & 0.000 & 0.000 & 0.000 & 0.000 & 0.000 & 0.0 \\
\hline C14: & 0.000 & 0.000 & 0.000 & 0.000 & 0.000 & 0.0 \\
\hline Total & 75.473 & 15.426 & 0.019 & 1.913 & 6.931 & 99.76 \\
\hline
\end{tabular}

Oxygenates: 0.013 Total C14t: 0.000

Total Unknowns:

0.225

Grand Total: 100.000

(in Mole Percent)

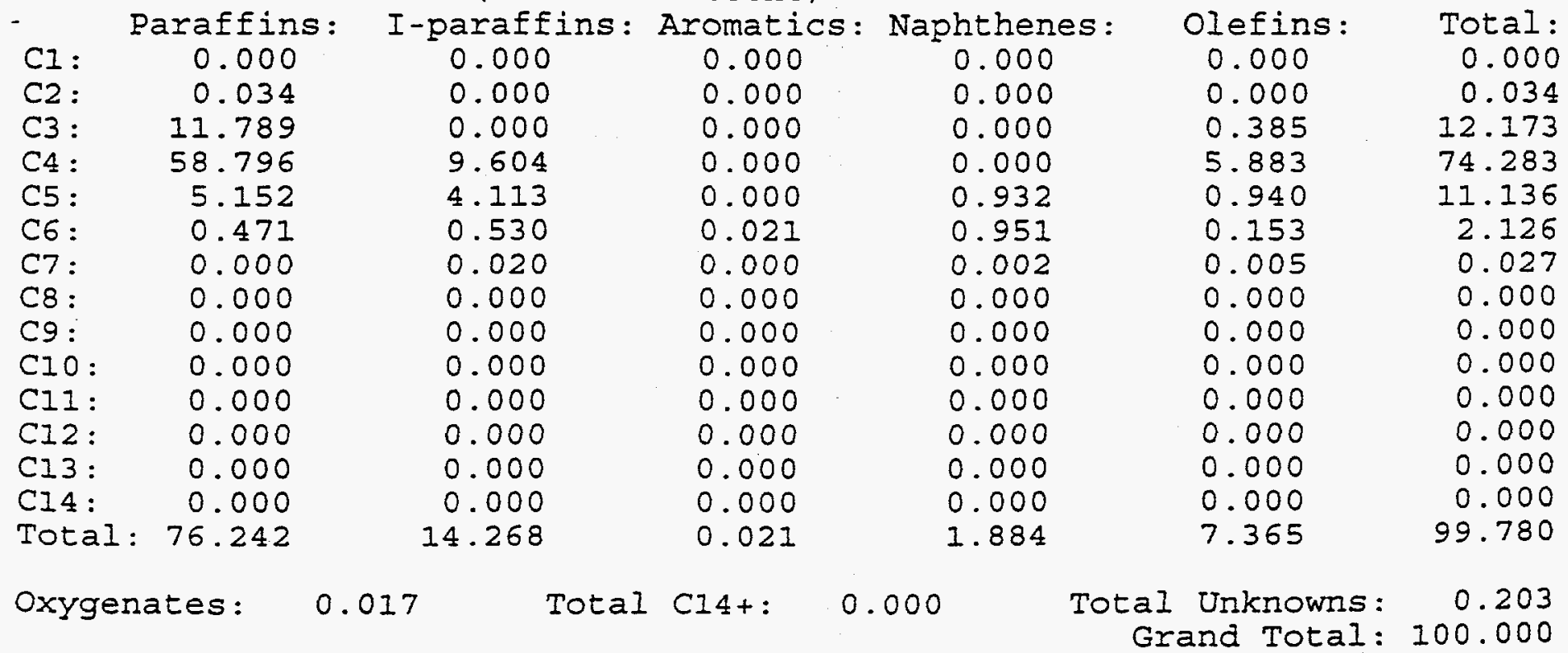




\section{CALEB BEET"I' HUUS'I'ULN}

IIO: 9ல்-UO517ن-U-HOUS-UUZ̈-نं

CID: CONSOLINC'

BID: HTI PB-03-9,10,11 CRUDE OIL/IBP-7O E

NID: $5.1870^{\circ}$

Date: 16-SEP-1996

Analyzed: 10/1/96 3:56 PM

Reported: 10-03-1996 13:02:33

Normalized to $100.00 \%$

Boiling Point Distribution Data

Wt. Percent off: deg.C.: $\operatorname{deg} . F$. :

$(0.5 \%)$
IBP $5.0 \%$
$10.0 \%$
$15.0 \%$
$20.0 \%$
$25.0 \%$
$30.0 \%$
$35.0 \%$
$40.0 \%$
$45.0 \%$
$50.0 \%$
$55.0 \%$
$60.0 \%$
$65.0 \%$
$70.0 \%$
$75.0 \%$
$80.0 \%$
$85.0 \%$
$90.0 \%$
$95.0 \%$
FBP $(99.5 \%)$

$$
\begin{array}{r}
-42.04 \\
-42.04 \\
-11.72 \\
-11.72 \\
-6.25 \\
-0.50 \\
-0.50 \\
-0.50 \\
-0.50 \\
-0.50 \\
-0.50 \\
-0.50 \\
-0.50 \\
-0.50 \\
-0.50 \\
-0.50 \\
0.88 \\
27.84 \\
36.06 \\
36.06 \\
80.72
\end{array}
$$

$$
\begin{array}{r}
-43.67 \\
-43.67 \\
10.90 \\
10.90 \\
20.75 \\
31.10 \\
31.10 \\
31.10 \\
31.10 \\
31.10 \\
31.10 \\
31.10 \\
31.10 \\
31.10 \\
31.10 \\
31.10 \\
33.58 \\
82.11 \\
96.91 \\
96.91 \\
177.30
\end{array}
$$

Vol. Percent off: deg.C.: $\quad \operatorname{deg} . F$. :

$-42.04$

$-42.04$

$-42.04$

$-11.72$

$-11.72$

$-0.50$

$-0.50$

$-0.50$

$-0.50$

$-0.50$

$-0.50$

$-0.50$

$-0.50$

$-0.50$

$-0.50$

$-0.50$

$-0.50$

20.05

31.15

36.06

80.72
$-43.67$

$-43.67$

$-43.67$

10.90

10.90

31.10

31.10

31.10

31.10

31.10

31.10

31.10

31.10

31.10

31.10

31.10

31.10

68.09

88.07

96.91

177.30

Research Octane Number $=108.79$

(Calculated from Individual Component Values)

$\begin{array}{cc}\text { Contribution to Total by } \\ \text { Paraffins: } & 81.07 \\ \text { Iso-paraffins: } & 17.32 \\ \text { Aromatics: } & 0.03 \\ \text { Naphthenes: } & 2.19 \\ \text { Olefins: } & 7.92 \\ \text { Oxygenates: } & 0.02\end{array}$

File: $5170 . \mathrm{DHA}$ 
CALEB BEET"I' HUUS'lUTW

IID: 9ல-UO5 $170-U-H O U S-U U 2-U U$

CID: CONSOLINC'

GID: HTI PB-03-9,10,11 CRUUE OIL/IBP-70 E

NIU: $51870^{\circ}$

Late: $16-S E P-1996$

Analyzed: 10/1/96 3:56 PM

Reported: 10-03-1996 13:02:33 Normalized to $100.00 \%$

Components Listed in Chromatographic Order

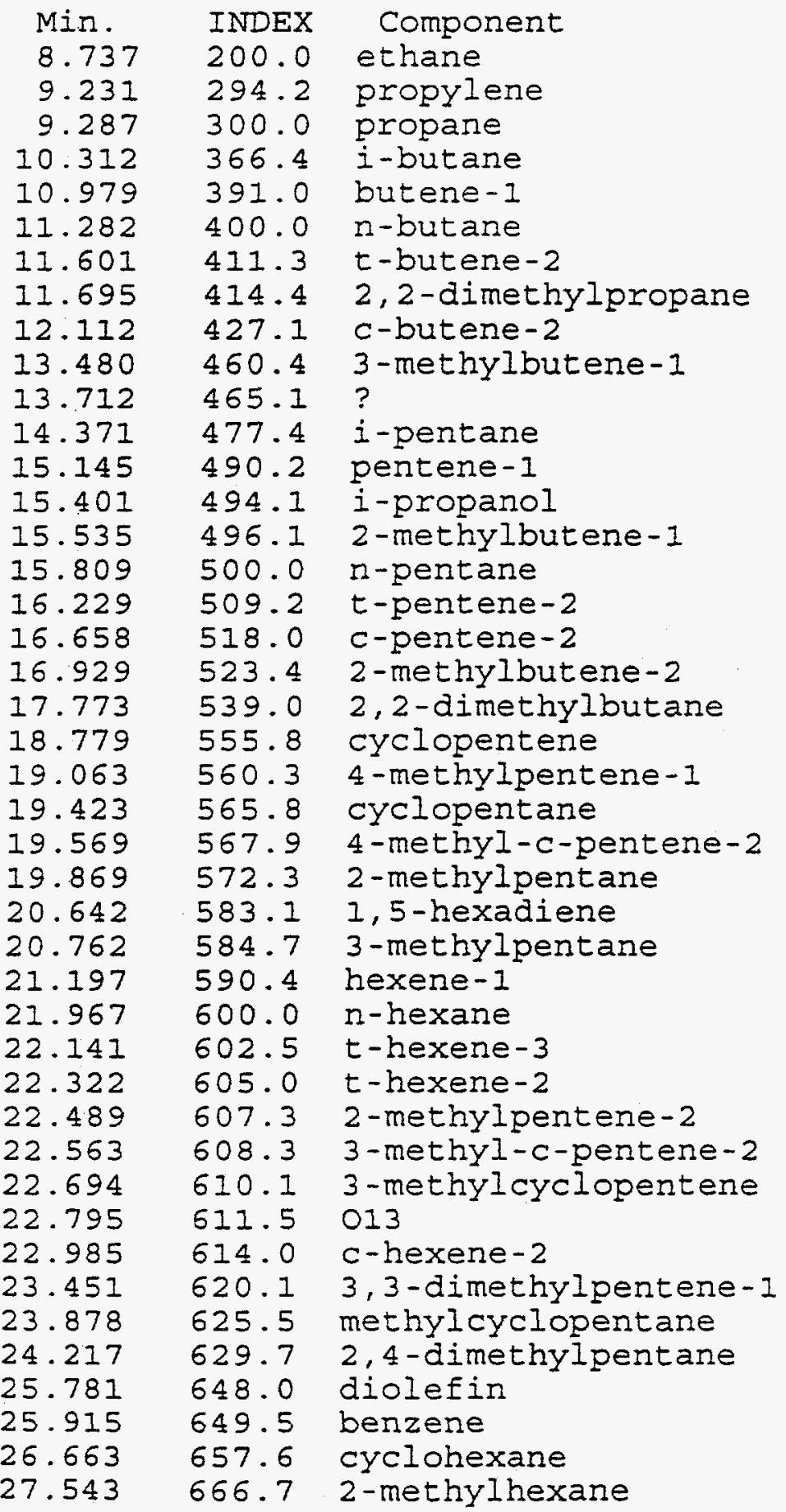

$\begin{array}{rrr}\text { Wt\% } & \text { VoI\% } & \text { Mol\% } \\ 0.018 & 0.030 & 0.034 \\ 0.277 & 0.318 & 0.385 \\ 8.900 & 10.313 & 11.789 \\ 9.557 & 9.947 & 9.604 \\ 2.701 & 2.632 & 2.812 \\ 58.508 & 58.626 & 58.796 \\ 1.573 & 1.510 & 1.638 \\ 0.040 & 0.039 & 0.033 \\ 1.377 & 1.285 & 1.433 \\ 0.108 & 0.100 & 0.090 \\ 0.244 & 0.225 & 0.203 \\ 5.041 & 4.718 & 4.081 \\ 0.259 & 0.235 & 0.216 \\ 0.018 & 0.013 & 0.017 \\ 0.102 & 0.091 & 0.085 \\ 6.364 & 5.894 & 5.152 \\ 0.276 & 0.247 & 0.230 \\ 0.145 & 0.129 & 0.121 \\ 0.127 & 0.111 & 0.105 \\ 0.008 & 0.007 & 0.005 \\ 0.108 & 0.081 & 0.092 \\ 0.023 & 0.020 & 0.016 \\ 1.119 & 0.870 & 0.932 \\ 0.044 & 0.038 & 0.030 \\ 0.503 & 0.447 & 0.341 \\ 0.012 & 0.010 & 0.008 \\ 0.271 & 0.237 & 0.184 \\ 0.031 & 0.026 & 0.021 \\ 0.694 & 0.611 & 0.471 \\ 0.016 & 0.014 & 0.011 \\ 0.021 & 0.018 & 0.015 \\ 0.011 & 0.010 & 0.008 \\ 0.016 & 0.013 & 0.011 \\ 0.007 & 0.005 & 0.005 \\ 0.008 & 0.006 & 0.005 \\ 0.011 & 0.009 & 0.008 \\ 0.008 & 0.006 & 0.005 \\ 0.677 & 0.525 & 0.470 \\ 0.007 & 0.006 & 0.004 \\ 0.020 & 0.016 & 0.014 \\ 0.028 & 0.019 & 0.021 \\ 0.692 & 0.516 & 0.481 \\ 0.021 & 0.018 & 0.012 \\ & & \end{array}$


Components Listed in Chromatographic Order

$\begin{array}{cccccc}\text { Min. } & \text { INDEX } & \text { Component } & \text { Wt\% } & \text { Vol\% } & \text { Mol\% } \\ 27.690 & 668.1 & 2,3 \text {-dimethylpentane } & 0.008 & 0.006 & 0.004 \\ 27.958 & 670.8 & 1,1 \text {-dimethylcyclopentane } & 0.003 & 0.002 & 0.002\end{array}$


APPENDIX 4

UNIVERSITY OF DELAWARE QUARTERLY REPORT

A4-1 


\title{
THE KINETICS OF COAL LIQUEFACTION DISTILLATION RESID CONVERSION
}

\author{
QUARTERLY REPORT \\ 7/16/96-10/15/96 \\ Michael T. Klein \\ Principal Investigator \\ William H. Calkins \\ Co-Principal Investigator \\ He Huang \\ Research Associate \\ and \\ Shaojie Wang \\ Visiting Scientist
}

Center For Catalytic Science and Technology

Department of Chemical Engineering

University of Delaware

Newark, Delaware 19716

Date Published

October 29,1996

Subcontract from CONSOL under DOE Contract DE-AC22-94PC9305 


\section{EXECUTIVE SUMMARY}

Hydroconversion runs on 15 resids supplied by CONSOL, Inc. have been completed using 3 to 1 tetralin to resid wt. ratio with and without $3 \mathrm{wt} \%$ sulfided molybdenum naphthenate catalyst at $420{ }^{\circ} \mathrm{C}$ for 30 minutes. Two of the resids from the Princeton laboratory and two catalyzed Wilsonville resids have still to be analyzed. The other resids showed 30.2 to $44.9 \%$ conversion to material boiling below $850^{\circ} \mathrm{F}$ in 30 minutes in the catalyzed experiments. This can be compared to uncatalyzed hydroconversion of the same materials of $15-23 \mathrm{wt} \%$ under the same conditions. The difference in these resids which can account for the variation of 30 up to $45 \%$ has not yet been investigated. Previous work using a different work up procedure showed that $0.9 \mathrm{wt} \%$ sulfided molybdenum naphthenate at the same times and temperature showed only a slight increase in conversion over the uncatalyzed system.

TGA tests run on solubilized and unsolubilized resid from both the catalyzed and thermal hydroconversion experiments show that there is little of no conversion of the unsolubilized residue to material boiling below $454^{\circ} \mathrm{C}\left(850^{\circ} \mathrm{F}\right)$. However, there is substantial conversion of the solubilized resid to lower boiling material.

The molecular modeling work is proceeding satisfactorily and the algorithm for the resid structure is in place. Necessary analytical work on the VPO molecular weights on the SARA fractions is almost complete. 


\section{QUARTERLY REPORT}

\section{HYDROCONVERSION OF COAL DERIVED RESIDS}

\section{Hydroconversion of resids}

Hydroconversion experiments have now been completed on all 15 resids supplied by CONSOL Inc. under the conditions agreed upon with CONSOL on May 17, 1996. Two of the resids from the Princeton laboratory and two catalyzed Wilsonville resids have still to be analyzed. The hydroconversion conditions were 30 minute runs at $420^{\circ} \mathrm{C}$ in 3 to 1 tetralin to resid wt ratio and 1500 psig hydrogen with and without using sulfided molybdenum naphthenate catalyst (as $3 \mathrm{wt} \%$ molybdenum based on resid charged). Each resid was also run at ambient temperature for comparative purposes.

Hydroconversion experiments using Shell Ni/Mo catalyst are still indeterminate pending the finding of a suitable method of conversion determination. Experiments have shown that the Shell catalyst itself changes under hydroprocessing conditions, making the use of ash tracer as a way of following conversion unreliable. We are considering alternative approaches to determining conversion when using that catalyst.

\section{Work up Procedure for Hydroprocessing Experiments}

The work-up of the runs is an extensive process. The reaction mixture is filtered and the filter cake is washed with cold methylene chloride into the filtrate. The methylene chloride is then distilled out of the filtrate at low temperature (below $42^{\circ} \mathrm{C}$ ). The low concentration of the tetralin in the methylene chloride distillate is determined by gas chromatography. The methylene chloride remaining and the tetralin in the resid filtrate are also determined by gas chromatography. SimDis TGA is performed on both the filtrate and the filter cake to allow the determination of the resid conversion to product boiling below $850^{\circ} \mathrm{F}$.

\section{Resid Conversion Kinetics}

Since the conversion is determined as the product of the amount of resid solubilized and the percentage of that solubilized product boiling below $850^{\circ} \mathrm{F}$, the conversion calculation is based on Equation:

$$
\text { Conversion }(<850 F)=T S F \times\left(1-\frac{850 F+}{R S F}\right)
$$

where the Tetralin Soluble Fraction (TSF) of resid (dry-ash-free basis) is determined by ash content in the solid residue after resid hydroprocessing; the Resid Soluble Fraction (RSF) in 
tetralin and the $850^{\circ} \mathrm{F}^{+}$fraction of the RSF are determined by running GC and SimDis TGA on the liquid products, respectively.

Table 1 shows the thermal and catalytic reactor runs made and the conditions used. Table 2 shows the solubilization of the thermal and catalyzed runs as well as the room temperature controls. Table 3 shows the increase in solubilization over the room temperature controls in the thermal and catalytic runs. There is a substantial solubility of all the resids in tetralin even at room temperature. The increases in solubilization over the room temperature experiments are only of the order of 10 to $20 \%$. The presence of a catalyst increases the solubilization only a small amount ( 1 to $4 \%$ ). Tables 4 and 5 show the conversions of resid to material boiling below $850^{\circ} \mathrm{F}$ for the thermal and catalyzed runs, respectively.

TGA on the solid residues using the ramp method shows that all the insoluble portion of the resids or reactor solid residues shows no material boiling below $850^{\circ} \mathrm{F}$. The hydroconversion of the resid apparently occurs only on the solublized resid. This conversion is only about 15 $20 \%$ for the thermal hydroconversion to $850^{\circ} \mathrm{F}$ with the tetralin donor solvent alone. On the other hand, in the presence of $3 \%$ molybdenum naphthenate catalyst, the conversion increases to 30.2 to $44.9 \%$ depending upon the resid. All of the 13 Wilsonville resids and two HTI resids have been run under thermal and catalytic conditions and have been analyzed. The recovery in processing the two very sticky HTI resids was relatively poor. Two more of the Wilsonville resids converted in the presence of catalyst and two HTI resids have not yet been completely analyzed.

Several difficulties have been experienced with the gas chromatograph, which is used in the analytical scheme. The latest problem relates to the drifting response factor of the FID detector. We are trying to avoid this problem by using an internal standard, and all the samples of filtrate and distillate are now being analyzed in this way. We believe, however, that the experimental errors in the conversion data presented in this report cannot be more than $5 \%$ (relative). With the use of internal standards, we expect to minimize them.

\section{MODELING OF RESID HYDROCONVERSION}

\section{Analysis of SARA Samples}

To date molecular weights have been determined for each of the aromatic, aliphatic and preasphaltene fractions of the SARA separations (Table 6). However, the resin samples which were dissolved in dichlorobenzene yielded results which are too high and these samples are unrecoverable. It will be assumed that these samples have a MW of 500 . The value of 500 falls within 2 experimental standard deviations of every measured resin, so this assumption should not 
lead to much error. To account for this approximation, the constraint in the structural model for these resids which requires the molecular weight of the resin fraction to match the experimentally determined value will be relaxed.

The molecular weights for the first six saturate fractions have also been determined. Only the remaining nine saturate fractions need to be determined. All other analytical information has been supplied by CONSOL, Inc.

\section{Modeling}

A molecular structure model for coal resid has been assembled (see Table 7 and Figure 1). Currently, a molecular representation of the first resid (W258 V-131B) is being optimized to the analytical properties of the initial feedstock. The molecules have been defined by their basic structural attributes (e.g. number of aromatic rings, number of thiophenic rings, number of sidechains, number of unit sheets, etc.) and a construction algorithm is presented in Figure 1. The attributes are represented by probability distribution functions ( $\mathrm{pdf}$ 's) which give the probability of finding a value or less of a given attribute. Table 7 lists the distributions used in this algorithm.

By stochastic (Monte Carlo) sampling of the pdf's, a molecular sample of a resid can be constructed. The properties of this representation can be compared to experimental values. The parameters of the pdf's are optimized to minimize the difference between the predicted and experimental values. The objective function used to optimize these parameters is:

$$
\begin{aligned}
& \chi^{2}=\left(\frac{H w t_{\text {exp }}-H w t_{p r e d}}{0.0016}\right)^{2}+\left(\frac{C w t_{\text {exp }}-C w t_{p r e d}}{0.0064}\right)^{2}+\left(\frac{O w t_{\text {exp }}-O w t_{p r e d}}{0.0150}\right)^{2}+ \\
& \left(\frac{N w t_{\text {exp }}-N w t_{\text {pred }}}{0.0011}\right)^{2}+\left(\frac{S w t_{\text {exp }}-S w t_{\text {pred }}}{0.0010}\right)^{2}+ \\
& \left(\frac{1}{\# \text { Comps }}\right)^{\# \text { Comps }}\left(\frac{\text { SaraWt }_{\text {exp }}-\text { SaraWt }_{\text {pred }}}{0.03}\right)^{2}+\left(\frac{\text { Harno }_{\text {exp }}-\text { Harno }_{\text {pred }}}{0.02}\right)^{2}+ \\
& \left(\frac{\text { HCarno }_{\text {exp }}-\text { HCarno }_{\text {pred }}}{0.02}\right)^{2}+\left(\frac{\text { Haano }_{\text {exp }}-\text { Haano }}{0.02}\right)^{2}+ \\
& \left(\frac{\text { HCano }_{\text {exp }}-\text { HCano }_{\text {pred }}}{0.02}\right)^{2}+\left(\frac{\text { Habno }_{\text {exp }}-\text { Habno }}{0.02}\right)^{2}+ \\
& \left(\frac{\text { HCbno }_{\text {exp }}-H_{C b n O_{p r e d}}}{0.02}\right)^{2}+\left(\frac{\text { Hgno }_{\text {exp }}-\text { Hgno pred }}{0.02}\right)^{2}+\left(\frac{\text { Phox }_{\text {exp }}-\text { Phox pred }}{0.02}\right)^{2}
\end{aligned}
$$

The numerator of each term represents the residual between the measured and predicted values. The denominator is a weighting factor equal to approximately one standard deviation. The 
weighting factor ensures that values which are measured with higher accuracy are represented more closely than values measure with lower accuracy.

The model will be fine-tuned with the first few resids. In particular, an investigation into the importance of conditional probability and into the approximation of the intersheet linkages will be made. It may be necessary to make some slight alterations to the current model to get an optimal representation. Later, some reaction product data may also be added to the objective function.

Sample molecules from this representation will be examined with a view to selecting model structures for determining hydroconversion rate constants in the SCTBR reactor. Such data is not essential to the functioning of the model. However, it could provide a useful starting point for the reaction rate constants.

\section{SUMMARY AND CONCLUSIONS}

1. A distinct increase in the production of material boiling below $850^{\circ} \mathrm{F}$ is observed in the presence of $3 \mathrm{wt} \%$ sulfided molybdenum naphthenate catalyst under the conditions used. This is substantially higher than that observed in hydroprocessing in the absence of catalyst (thermal) or when only $0.9 \mathrm{wt} \%$ of the same catalyst is used.

2. Little or no conversion of the unsolubilized resid to lower boiling material appears to occur even in the presence of the catalyst.

3. The solubilization of the resid in tetralin is substantial even at room temperature. It increases only 10 to $20 \mathrm{wt} \%$ under hydroprocessing conditions without catalyst. There is very little increase in solubilization in the presence of catalyst. However, the breakdown of the soluble resid into low boiling material depends strongly on the catalyst.

4. Conversions of various resids under catalytic conditions have varied from 30 to $45 \mathrm{wt} \%$. These values are being correlated with analytical data of the resids.

5. A molecular structure model for coal derived resid has been assembled and is being optimized.

6. Molecular weight determination of the various SARA fractions is almost complete. 


\section{FUTURE PROGRAM}

1. In consultation with CONSOL Inc., one or more selected resids will be hydroprocessed over a range of conditions, i.e., time, temperature, tetralin to resid ratio, catalyst concentration, hydrogen pressure and solvent type (including Wilsonville recycle solvent) to establish what conversion of recycle resid can be expected under actual process conditions.

2. An effort will be made to correlate conversion data with the available structural information (NMR, elemental analysis etc.).

3. If time permits, an effort will be made to relate hydroprocessing performance in the presence of molybdenum naphthenate catalyst to that of the use of sulfided $\mathrm{Ni}-\mathrm{Mo} / \mathrm{Al}_{2} \mathrm{O}_{3}$ catalysts used at Wilsonville and the other promising catalysts developed in recent years.

4. If time permits, some rate constants of selected model compounds will be determined for hydroprocessing to assist in the molecular modeling work.

\section{PUBLICATIONS}

Three papers were presented to the New Orleans National Meeting of the American Chemical Society in the Spring of 1996 that relate in part to work done under this project. They are:

1. He Huang, Keyu Wang, Shaojie Wang, M.T. Klein, and W.H. Calkins 'Applications of the Thermogravimetric Analysis in the Study of Fossil Fuels', Prepr. Pap. - Am. Chem. Soc., Div. Fuel Chem. 1996, 41(1), 1.

2. Keyu Wang, Shaojie Wang, He Huang, M.T. Klein, and W.H. Calkins 'A Novel Smoothing Routine for the Data Processing in Thermogravimetric Analysis', Prepr. Pap. - Am. Chem. Soc., Div. Fuel Chem. 1996, 41(1), 27.

3. He Huang, Keyu Wang, Shaojie Wang, M.T. Klein, and W.H. Calkins 'Distillation of Liquid Fuels by Thermogravimetry', Prepr. Pap. - Am. Chem. Soc., Div. Fuel Chem. 1996, 41(1), 87. 
Table 1 Reactor Runs

\begin{tabular}{|c|c|c|c|c|c|}
\hline \multicolumn{2}{|c|}{ Sample } & \multicolumn{2}{|c|}{ Thermal } & \multicolumn{2}{|c|}{ Catalytic } \\
\hline & & Run \# & $\mathrm{T}, \mathrm{C}$ & Run \# & $\mathrm{T}, \mathrm{C}$ \\
\hline Resid A & W258V-131B & C096 & 425 & $\mathrm{C} 109$ & 422 \\
\hline Resid B & W259R-1235 & $\mathrm{C} 097$ & 421 & $\mathrm{C} 110$ & 417 \\
\hline Resid C & W259V-131B & C098 & 422 & $\mathrm{C} 123$ & 419 \\
\hline Resid D & W261V-131B & C099 & 426 & C124 & 420 \\
\hline Resid E & W262R-1235 & $\mathrm{C} 100$ & 420 & $\mathrm{C} 125$ & 421 \\
\hline Resid F & W262V-1067 & $\mathrm{C} 101$ & 422 & $\mathrm{C} 126$ & 418 \\
\hline Resid G & W262V-131B & $\mathrm{C} 102$ & 422 & $\mathrm{C} 127$ & 420 \\
\hline Resid H & W260V-131B & $\mathrm{C} 103$ & 420 & C128 & 419 \\
\hline Resid I & W261V-1067 & $\mathrm{C} 104$ & 420 & $\mathrm{C} 129$ & 420 \\
\hline Resid J & W259V-1067 & $\mathrm{C} 105$ & 421 & $\mathrm{C} 130$ & 420 \\
\hline Resid K & W260R-1235 & C106 & 421 & C131 & 419 \\
\hline Resid L & W260V-1067 & $\mathrm{C} 107$ & 418 & C132 & 421 \\
\hline Resid M & W261R-1235 & $\mathrm{C} 108$ & 418 & $\mathrm{C} 133$ & 418 \\
\hline Resid N & HTI POC-01, O-43 & $\mathrm{C} 134$ & 423 & $\mathrm{C} 137$ & 421 \\
\hline Resid O & HTI POC-02, O-43 & $\mathrm{C} 135$ & 422 & $\mathrm{C} 136$ & 423 \\
\hline \multicolumn{2}{|c|}{ Thermal: $30 \mathrm{~min} ; 1500$ psig H2 } & & & & \\
\hline \multicolumn{6}{|c|}{ Catalytic: $30 \mathrm{~min} ; 1500 \mathrm{psig} \mathrm{H} 2 ; 3 \mathrm{wt} \% \mathrm{Mo}$ (molybdenum naphthenate) based on the amount of } \\
\hline \multicolumn{3}{|c|}{ the resid chaged; ca. $3 \mathrm{~g}$ of methyl disulfide } & & & \\
\hline
\end{tabular}


Table 2 Tetralin Soluble Fraction of Resid

\begin{tabular}{|c|c|c|c|c|c|c|c|c|c|c|c|}
\hline \multicolumn{2}{|c|}{ Sample } & \multirow[t]{2}{*}{ Ash } & \multicolumn{3}{|c|}{ Thermal } & \multicolumn{3}{|c|}{ Catalytic } & \multicolumn{3}{|c|}{ Control } \\
\hline & & & Run \# & Ash & TSF & Run \# & Ash & TSF & Run \# & Ash & TSF \\
\hline & & & & & & & & & & & \\
\hline Resid A & W258V-131B & 17.0 & $\mathrm{C} 096$ & 50.1 & 79.7 & $\mathrm{C} 109$ & 51.5 & 80.7 & $\mathrm{C} 091$ & 40.2 & 69.6 \\
\hline Resid B & W259R-1235 & 8.7 & $\mathrm{C} 097$ & 33.5 & 81.0 & $\mathrm{C} 110$ & 40.0 & 85.6 & $\mathrm{C} 111$ & 21.9 & 65.8 \\
\hline Resid C & $\mathrm{W} 259 \mathrm{~V}-131 \mathrm{~B}$ & 8.5 & $\mathrm{C} 098$ & 35.0 & 82.7 & $\mathrm{C} 123$ & 41.4 & 86.8 & $\mathrm{C} 112$ & 24.6 & 71.5 \\
\hline Resid D & W261V-131B & 9.9 & C099 & 45.8 & 87.1 & $\mathrm{C} 124$ & 54.9 & 91.0 & $\mathrm{C} 113$ & 29.7 & 74.1 \\
\hline Resid E & W262R-1235 & 15.6 & $\mathrm{C} 100$ & 43.5 & 76.0 & $\mathrm{C} 125$ & 44.2 & 76.6 & C114 & 32.6 & 61.7 \\
\hline Resid F & W262V-1067 & 17.5 & $\mathrm{C} 101$ & 47.8 & 76.9 & $\mathrm{C} 126$ & 49.4 & 78.3 & $\mathrm{C} 115$ & 38.6 & 66.4 \\
\hline Resid G & W262V-131B & 15.9 & $\mathrm{C} 102$ & 46.5 & 78.3 & $\mathrm{C} 127$ & 48.3 & 79.8 & $\mathrm{C} 116$ & 39.1 & 70.6 \\
\hline Resid H & W260V-131B & 15.2 & $\mathrm{C} 103$ & 46.6 & 79.5 & $\mathrm{C} 128$ & 50.9 & 82.7 & $\mathrm{C} 117$ & 38.3 & 71.2 \\
\hline Resid I & W261V-1067 & 15.9 & $\mathrm{C} 104$ & 50.8 & 81.8 & $\mathrm{C} 129$ & 56.6 & 85.5 & C118 & 37.9 & 69.2 \\
\hline Resid J & W259V-1067 & 10.2 & $\mathrm{Cl05}$ & 38.7 & 82.0 & $\mathrm{C} 130$ & 43.8 & 85.4 & $\mathrm{C} 119$ & 29.0 & 72.2 \\
\hline Resid K & W260R-1235 & 17.2 & $\mathrm{C} 106$ & 49.0 & 78.4 & $\mathrm{C} 131$ & 52.1 & 80.8 & $\mathrm{C} 120$ & 35.6 & 62.4 \\
\hline Resid L & W260V-1067 & 16.7 & $\mathrm{C} 107$ & 51.3 & 81.0 & $\mathrm{C} 132$ & 53.5 & 82.6 & $\mathrm{C} 121$ & 37.4 & 66.6 \\
\hline Resid M & W261R-1235 & 13.7 & $\mathrm{C} 108$ & 45.6 & 81.1 & $\mathrm{C} 133$ & 53.7 & 86.3 & $\mathrm{C} 122$ & 30.9 & 64.5 \\
\hline Resid N & HTI POC-01, O-43 & 0.4 & $\mathrm{Cl34}$ & 33.0 & 99.2 & $\mathrm{C} 137$ & 36.4 & 99.3 & $\mathrm{C} 139$ & 33.4 & 99.2 \\
\hline Resid O & HTI POC-02, O-43 & 4.1 & $\mathrm{C} 135$ & 38.0 & 93.1 & $\mathrm{C} 136$ & 48.3 & 95.4 & $\mathrm{C} 138$ & 37.4 & 92.9 \\
\hline \multicolumn{3}{|c|}{ Thermal: $420 \mathrm{C} ; 30 \mathrm{~min} ; 1500$ psig H2 } & & & & & & & & & \\
\hline \multicolumn{4}{|c|}{ Catalytic: $420 \mathrm{C} ; 30 \mathrm{~min} ; 1500$ psig H2; $3 \mathrm{wt} \% \mathrm{Mo}$} & & & & & & & & \\
\hline \multicolumn{3}{|c|}{ Control: $25 \mathrm{C} ; 10 \mathrm{~min} ; 1500$ psig H2 } & & & & & & & & & \\
\hline \multicolumn{5}{|c|}{ TSF: Tetralin Soluble Fraction of resid, wt\% (daf basis) } & & & & & & & \\
\hline
\end{tabular}


Table 3 Differences of the Tetralin Soluble Fraction of resid at various processing conditions

\begin{tabular}{|c|c|c|c|c|}
\hline \multirow[t]{2}{*}{ Sample } & \multirow[t]{2}{*}{ Name } & \multicolumn{3}{|c|}{ Tetralin Soluble Fraction of resid, daf basis } \\
\hline & & Thermal - Control & Catalytic - Control & Catalytic - Thermal \\
\hline Resid A & W258V-131B & 10.1 & 11.2 & 1.1 \\
\hline Resid B & W259R-1235 & 15.2 & 19.8 & 4.6 \\
\hline Resid C & W259V-131B & 11.2 & 15.3 & 4.1 \\
\hline Resid D & W261V-131B & 13.0 & 16.9 & 4.0 \\
\hline Resid E & W262R-1235 & 14.3 & 14.9 & 0.6 \\
\hline Resid F & $\mathrm{W} 262 \mathrm{~V}-1067$ & 10.5 & 11.9 & 1.4 \\
\hline Resid G & W262V-131B & 7.8 & 9.3 & 1.5 \\
\hline Resid H & W260V-131B & 8.3 & 11.5 & 3.2 \\
\hline Resid I & W261V-1067 & 12.6 & 16.4 & 3.8 \\
\hline Resid J & W259V-1067 & 9.8 & 13.2 & 3.4 \\
\hline Resid K & W260R-1235 & 15.9 & 18.4 & 2.5 \\
\hline Resid L & W260V-1067 & 14.4 & 16.1 & 1.6 \\
\hline Resid M & W261R-1235 & 16.5 & 21.8 & 5.3 \\
\hline Resid N & HTI POC-01, O-43 & 0.0 & 0.1 & 0.1 \\
\hline Resid O & HTI POC- $02,0-43$ & 0.2 & 2.6 & 2.4 \\
\hline & & & & \\
\hline \multicolumn{5}{|c|}{ Thermal: $420 \mathrm{C} ; 30 \mathrm{~min} ; 1500$ psig H2 } \\
\hline \multicolumn{5}{|c|}{ Catalytic: $420 \mathrm{C} ; 30 \mathrm{~min} ; 1500 \mathrm{psig} \mathrm{H} 2 ; 3 \mathrm{wt} \%$ of $\mathrm{Mo}$} \\
\hline \multicolumn{5}{|c|}{ Control: $25 \mathrm{C} ; 10 \mathrm{~min} ; 1500 \mathrm{psig} \mathrm{H} 2$} \\
\hline & & & & \\
\hline
\end{tabular}


Table 4 Conversion of thermal hydroprocessing of resid

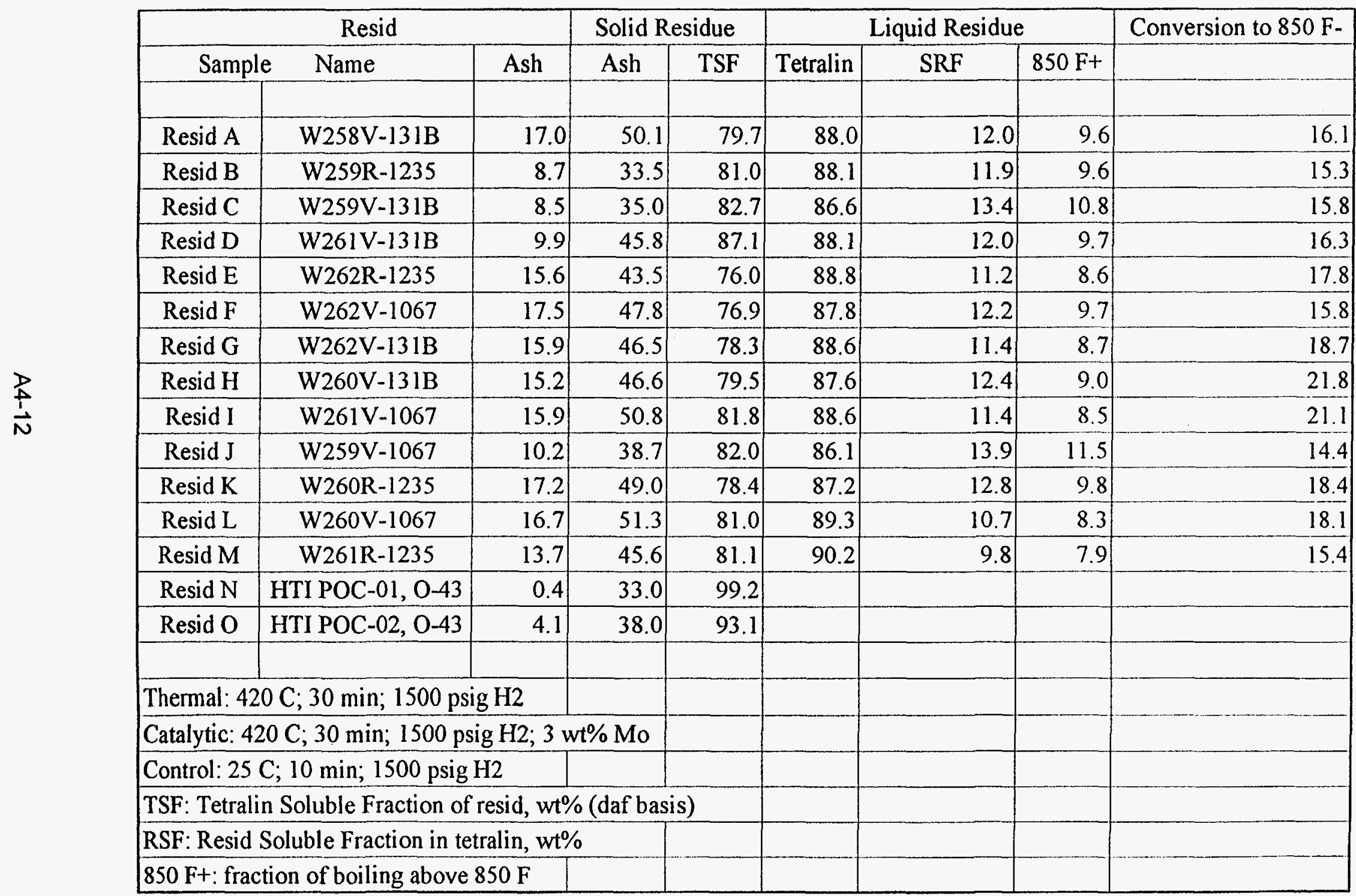


Table 5 Conversion of catalytic hydroprocessing of resid

\begin{tabular}{|c|c|c|c|c|c|c|c|c|}
\hline \multicolumn{3}{|c|}{ Resid } & \multicolumn{2}{|c|}{ Solid Residue } & \multicolumn{3}{|c|}{ Liquid Residue } & \multirow[t]{2}{*}{ Conversion } \\
\hline Samp & Name & Ash & Ash & SF & Tetralin & SR in Tetralin & $850 \mathrm{~F}+$ & \\
\hline Resid A & $\mathrm{W} 258 \mathrm{~V}-131 \mathrm{~B}$ & 17.0 & 51.5 & 80.7 & 82.4 & 17.6 & 9.9 & 35.3 \\
\hline Resid B & W259R-1235 & 8.7 & 40.0 & 85.6 & 80.3 & 19.7 & 11.2 & 36.9 \\
\hline Resid C & W259V-131B & 8.5 & 41.4 & 86.8 & 81.9 & 18.1 & 11.5 & 31.7 \\
\hline Resid D & W261V-131B & 9.9 & 54.9 & 91.0 & 77.3 & 22.7 & 14.1 & 34.4 \\
\hline Resid E & W262R-1235 & 15.6 & 44.2 & 76.6 & 81.4 & 18.6 & 11.3 & 30.2 \\
\hline Resid F & W262V-1067 & 17.5 & 49.4 & 78.3 & 80.2 & 19.8 & 10.7 & 36.2 \\
\hline Resid G & W262V-131B & 15.9 & 48.3 & 79.8 & 80.0 & 20.0 & 11.5 & 34.1 \\
\hline Resid H & W260V-131B & 15.2 & 50.9 & 82.7 & 75.4 & 24.6 & 11.3 & 44.9 \\
\hline Resid I & W261 V-1067 & 15.9 & 56.6 & 85.5 & 76.0 & 24.0 & 11.9 & 43.0 \\
\hline Resid J & W259V-1067 & 10.2 & 43.8 & 85.4 & 77.2 & 22.8 & 11.9 & 40.8 \\
\hline Resid K & W260R-1235 & 17.2 & 52.1 & 80.8 & 78.9 & 21.1 & 12.4 & 33.5 \\
\hline Resid L & W260V-1067 & 16.7 & 53.5 & 82.6 & & & & \\
\hline Resid M & W261R-1235 & 13.7 & 53.7 & 86.3 & & & & \\
\hline Resid N & HTI POC-01, 0-43 & 0.4 & 36.4 & 99.3 & & & & \\
\hline Resid O & HTI POC-02, O-43 & 4.1 & 48.3 & 95.4 & & & & \\
\hline & & & & & & & & \\
\hline \multicolumn{3}{|c|}{ Thermal: $420 \mathrm{C} ; 30 \mathrm{~min} ; 1500$ psig H2 } & & & & & & \\
\hline \multicolumn{4}{|c|}{ Catalytic: $420 \mathrm{C} ; 30 \mathrm{~min} ; 1500 \mathrm{psig} \mathrm{H2;3}$ wt\% Mo } & & & & & \\
\hline \multicolumn{3}{|c|}{ Control: $25 \mathrm{C} ; 10 \mathrm{~min} ; 1500$ psig H2 } & & & & & & \\
\hline \multicolumn{4}{|c|}{ TSF: Tetralin Soluble Fraction, wt $\%$ (daf basis) } & & & & & \\
\hline \multicolumn{4}{|c|}{ RSF: Resid Soluble Fraction in tetralin, wt $\%$} & & & & & \\
\hline \multicolumn{3}{|c|}{$850 \mathrm{~F}+$ : fraction of boiling above $850 \mathrm{~F}$} & & & & & & \\
\hline
\end{tabular}


Table 6: VPO results

\begin{tabular}{|l|l|l|l|l|l|}
\hline $\begin{array}{l}\text { Sample } \\
\text { Number }\end{array}$ & Saturate MW & Aromatic MW & Resin MW & $\begin{array}{l}\text { Asphaltene } \\
\text { MW }\end{array}$ & $\begin{array}{l}\text { Pre-Asphaltene } \\
\text { MW }\end{array}$ \\
\hline 1 & 288 & 320 & $676^{*}(500)$ & 778 & 988 \\
\hline 2 & 250 & 274 & $613^{*}(500)$ & 926 & 1060 \\
\hline 3 & 295 & 319 & 539 & 680 & 919 \\
\hline 4 & 305 & 362 & $718^{*}(500)$ & 595 & 988 \\
\hline 5 & 443 & 493 & $789^{*}(500)$ & 864 & 1037 \\
\hline 6 & 347 & 420 & $(500)$ & 789 & 1201 \\
\hline 7 & & 348 & 426 & 708 & 1202 \\
\hline 8 & & 425 & 535 & 686 & 1025 \\
\hline 9 & & 323 & 499 & 759 & 1087 \\
\hline 10 & & 430 & 520 & 657 & 895 \\
\hline 11 & & 386 & 479 & 760 & 829 \\
\hline 12 & & 413 & 502 & 653 & 1114 \\
\hline 13 & & 336 & 542 & 745 & 1107 \\
\hline 14 & & 356 & 501 & 718 & 1087 \\
\hline 15 & & 345 & 515 & 743 & 997 \\
\hline
\end{tabular}

* MWs determined using dichlorobenzene as solvent, results likely too high. 
Table 7 Attribute distributions used to construct a molecular representation for coal resid.

\begin{tabular}{|l|l|l|}
\hline Distribution & Name & Used to construct fractions: \\
\hline Paraffin Length & npdis & Paraffins \\
\hline \# of Naphthenic Rings & nrdis & Naphthenics \\
\hline \# of Sidechains & nscdis & Naphthenics, Aromatics/Resins, Asphaltenes, Preasphaltenes \\
\hline Length of Naphthenic Sidechains & nsldis & Naphthenics \\
\hline Length of Aromatic Sidechains & asldis & Aromatics/Resins, Asphaltenes, Preasphaltenes \\
\hline Naphthenic \# of Unit Sheets & ndpdis & Naphthenics \\
\hline Resin \# of Unit Sheets & rdpdis & Aromatics/Resins \\
\hline Asphaltene \# of Unit Sheets & adpdis & Asphaltenes \\
\hline Preasphaltene \# of Unit Sheets & pdpdis & Preasphaltenes \\
\hline \# of Aromatic Rings & ardis & Aromatics/ Resins, Asphaltenes, Preasphaltenes \\
\hline $\begin{array}{l}\text { \# of Naphthenic Rings on an Aromatic } \\
\text { Core }\end{array}$ & nardis & Aromatics/Resins, Asphaltenes, Preasphaltenes \\
\hline \# of Phenolic Oxygens per unit sheet & frphh & Aromatics/Resins, Asphaltenes, Preasphaltenes \\
\hline Intersheet Connections & & \\
\hline Fraction of Oxygen Connections & 0.25 & Resins, Asphaltenes, Preasphaltenes \\
\hline Fraction of Sulfur Connections & 0.25 & Resins, Asphaltenes, Preasphaltenes \\
\hline Fraction of Methylene Connections & $0.50: 0.25$ & Napthenics:Aromatics/Resins, Asphaltenes, Preasphaltenes \\
\hline Fraction of Biphenyl Connections & $0.50: 0.25$ & Napthenics:Aromatics/ Resins, Asphaltenes, Preasphaltenes \\
\hline Heteroatomic Rings & & \\
\hline Fraction of Oxygen Rings & frorings & Resins, Asphaltenes, Preasphaltenes \\
\hline Fraction of Sulfur Rings & frsrings & Resins, Asphaltenes, Preasphaltenes \\
\hline $\begin{array}{l}\text { Fraction of Nitrogen Rings } \\
\text { (5 and 6 members in a 2 10 I ratio) }\end{array}$ & frnings & Resins, Asphaltenes, Preasphaltenes \\
\hline Fraction of no Heteroatomic Rings & frhcrings & Resins, Asphaltenes, Preasphaltenes \\
\hline
\end{tabular}




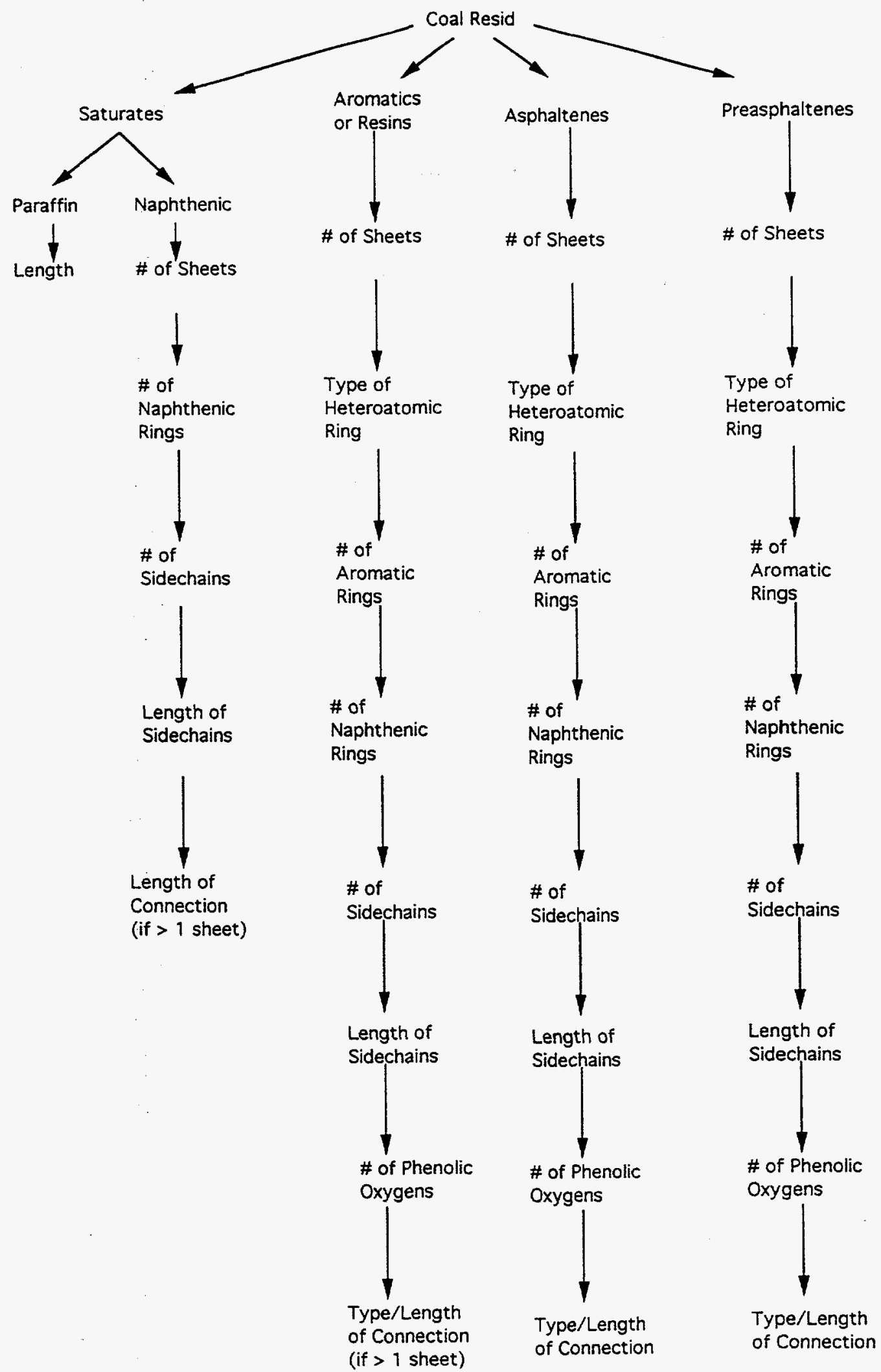

Figure 1: Coal Resid Construction Algorithm 